\title{
Cognitive dysfunction
}

Citation for published version (APA):

Geijselaers, S. L. C. (2016). Cognitive dysfunction: At the crossroads of glucose metabolism and vascular function. [Doctoral Thesis, Maastricht University]. Maastricht University.

https://doi.org/10.26481/dis.20161005sg

Document status and date:

Published: 01/01/2016

DOI:

$10.26481 /$ dis.20161005sg

Document Version:

Publisher's PDF, also known as Version of record

\section{Please check the document version of this publication:}

- A submitted manuscript is the version of the article upon submission and before peer-review. There can be important differences between the submitted version and the official published version of record.

People interested in the research are advised to contact the author for the final version of the publication, or visit the DOI to the publisher's website.

- The final author version and the galley proof are versions of the publication after peer review.

- The final published version features the final layout of the paper including the volume, issue and page numbers.

Link to publication

\footnotetext{
General rights rights.

- You may freely distribute the URL identifying the publication in the public portal. please follow below link for the End User Agreement:

www.umlib.nl/taverne-license

Take down policy

If you believe that this document breaches copyright please contact us at:

repository@maastrichtuniversity.nl

providing details and we will investigate your claim.
}

Copyright and moral rights for the publications made accessible in the public portal are retained by the authors and/or other copyright owners and it is a condition of accessing publications that users recognise and abide by the legal requirements associated with these

- Users may download and print one copy of any publication from the public portal for the purpose of private study or research.

- You may not further distribute the material or use it for any profit-making activity or commercial gain

If the publication is distributed under the terms of Article $25 \mathrm{fa}$ of the Dutch Copyright Act, indicated by the "Taverne" license above, 


\section{COGNITIVE DYSFUNCTION:}

AT THE CROSSROADS OF GLUCOSE METABOLISM AND VASCULAR FUNCTION 
(C) Stefan L.C. Geijselaers, Nijmegen 2016

Cover picture: "Human head thinking" by Art4All

Printed by: Ipskamp Drukkers B.V.

ISBN: 978-94-028-0305-1 


\title{
COGNITIVE DYSFUNCTION: \\ AT THE CROSSROADS OF GLUCOSE METABOLISM AND VASCULAR FUNCTION
}

\author{
PROEFSCHRIFT \\ ter verkrijging van de graad van doctor \\ aan de Universiteit Maastricht, \\ op gezag van de Rector Magnificus, Prof. dr. Rianne M. Letschert, \\ volgens het besluit van het College van Decanen, \\ in het openbaar te verdedigen op \\ woensdag 5 oktober 2016 om 10.00 uur \\ door \\ Stefan Leonardus Cristina Geijselaers
}

Geboren op 19 oktober 1987 te Maastricht 


\section{Promotores}

Prof. dr. C.D.A. Stehouwer

Prof. dr. GJ Biessels (Universitair Medisch Centrum Utrecht, Utrecht)

\section{Copromotor \\ Dr. S.J.S. Sep}

\section{Beoordelingscommissie}

Prof. dr. H. ten Cate, voorzitter

Prof. dr. I.C.W. Arts

Prof. dr. J.M. Dekker (VU Universitair Medisch Centrum, Amsterdam)

Dr. E. van den Berg (Erasmus Universitair Medisch Centrum, Rotterdam)

Prof. dr. R.J. van Oostenbrugge

Financial support by the Dutch Heart Foundation for the publication of this thesis is gratefully acknowledged.

Additional financial support for the publication of this thesis was generously provided by Alzheimer Nederland (Amersfoort, the Netherlands), Boehringer Ingelheim, and Novo Nordisk.

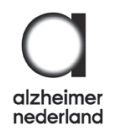




\section{Contents}

$\begin{array}{lll}\text { Chapter } 1 & \text { General introduction } & 7\end{array}$

Chapter 2 The role of hyperglycaemia, insulin resistance, and blood pressure in diabetes-associated differences in cognitive performance: The Maastricht Study

Chapter 3 Carotid stiffness is associated with impairment of cognitive performance in individuals with and without type 2 diabetes:

The Maastricht Study

(Atherosclerosis, 2016)

Chapter 4 Carotid circumferential wall stress is not associated with cognitive performance: The Maastricht Study

Chapter 5 Glucose regulation, cognition, and brain MRI in type 2 diabetes: a systematic review

(The Lancet Diabetes \& Endocrinology, 2015)

Chapter 6 Insulin resistance and cognitive performance in type 2 diabetes: 205 The Maastricht Study

Chapter 7 Association of cerebrospinal fluid insulin with cognitive performance and cerebrospinal fluid biomarkers of Alzheimer's disease: The Pearl Neurodegenerative Diseases

Chapter 8 Summary and General Discussion

Nederlandstalige samenvatting

Dankwoord

285

Curriculum Vitae 


\section{Chapter 1}

GENERAL INTRODUCTION 
Over the past two centuries, global life expectancy has more than doubled, and there is no indication that this trend of increasing longevity is slowing down. ${ }^{1}$ Indeed, from 2000 to 2012, life expectancy at birth in the Netherlands has increased by 3.6 years for men (from 75.5 to 79.1 years) and 2.2 years for women (from 80.6 to 82.8 years), ${ }^{2}$ which equals an astonishing 7 hours of life expectancy gained per day for men, and 4 hours per day for women. ${ }^{2,3}$ Unfortunately, longevity is not interchangeable with living 'happily ever after' and comes at a price. As we age, our brain undergoes structural changes that will negatively impact our cognitive abilities, ${ }^{4}$ that is, our ability to perceive and process information. ${ }^{5}$ The cognitive abilities believed to be most vulnerable to age-related decline are memory function, information processing speed, and executive function and attention, ${ }^{6}$ the latter of which is essential for goal-directed behaviour, such as planning, problem-solving, and decision-making. ${ }^{7}$

Although nearly everyone who lives into old age experiences a certain amount of cognitive decline, not everyone will develop serious cognitive problems such as dementia. Research in recent years has identified several modifiable risk factors that increase the likelihood of developing cognitive problems, including type 2 diabetes. ${ }^{8}$ The prevalence of diabetes has increased by 50 to $65 \%$ in the Netherlands over the last decade, ${ }^{9}$ which, combined with the ageing of the population, is likely to markedly increase the number of individuals suffering from cognitive problems in the near future. Over the next 40 years, for example, the number of Alzheimer's cases is projected to triple, affecting about 132 million individuals by the year $2050 .{ }^{10}$ Cognitive problems pose a tremendous burden to those who are affected, as cognitive abilities are essential to initiate, perform, and evaluate both routine and complex activities. It is this loss of autonomy and independence that makes cognitive decline the most feared aspect of growing old. ${ }^{11}$ At the same time, cognitive problems have a large impact on society in terms of direct and indirect health care costs, as, for example, illustrated by estimated annual societal costs of 50,000 euro per dementia case, ${ }^{12}$ further highlighting the need for interventions that can effectively prevent, reduce, or even reverse cognitive decline.

\section{Type 2 diabetes and cognitive performance}

Back in 1922, Miles and $\operatorname{Root}^{13}$ were the first to report a link between diabetes and cognitive performance. Based on their own experience with diabetic patients complaining of problems with memory and concentration, their investigation of cognitive performance in individuals with and without diabetes revealed that those with diabetes performed worse on measures of memory and information processing speed. ${ }^{13}$ Since then, an ever-growing number of studies and meta-analyses have confirmed that type 2 diabetes is associated with a variety of cognitive problems, ranging from subtle cognitive decrements to mild cognitive impairment and dementia. ${ }^{8,14} \mathrm{~A}$ recent meta- 
analysis covering 19 studies revealed that having type 2 diabetes roughly doubles the risk of developing dementia, ${ }^{15}$ with a relative risk of 2.48 (95\% confidence interval 2.08 2.96) for vascular dementia and 1.46 (1.20-1.77) for Alzheimer's disease. It has even been calculated that currently one in every 10 to 15 cases of dementia is attributable to diabetes. $^{16}$

At present, little is known about the factors underlying diabetes-associated decrements in cognitive performance. Given that type 2 diabetes usually develops in the context of an adverse cardiovascular risk profile, it has been frequently suggested (eg, $\left.{ }^{14,17}\right)$ that both metabolic and vascular factors are likely to play a role. Although never formally tested, this hypothesis is indirectly supported by evident associations of measures of glucose metabolism, such as fasting glucose or insulin concentrations, or vascular risk factors, such as hypertension and hyperlipidaemia, with cognitive performance in the general population $\left(\mathrm{eg},{ }^{18-20}\right)$.

Is there reason to believe that glucose metabolism plays a role in diabetes-associated cognitive problems?

Type 2 diabetes is characterised by hyperglycaemia and abnormal insulin signalling. ${ }^{21}$ Experimental research provides evidence indicating that both of these abnormalities in glucose metabolism directly affect brain function. Hyperglycaemia increases oxidative stress, mainly through mitochondrial overproduction of superoxide. ${ }^{22}$ Excessive superoxide generation, in turn, is known to induce multiple (intracellular) biochemical changes, ${ }^{22-24}$ including glycation of important structural and functional proteins, which may give rise to neuronal dysfunction and cell death. ${ }^{25}$ At the same time, these biochemical changes may cause micro- and macrovascular damage ${ }^{23,24}$ as chronic hyperglycaemia has been associated with blood-brain barrier dysfunction ${ }^{26}$ and reduced cerebral blood flow. ${ }^{27}$

Traditionally, the brain was considered an insulin insensitive organ. In recent years, however, the central effects of insulin have become increasingly apparent, including its role in cognition. ${ }^{28}$ The mechanisms through which insulin may affect cognitive performance are diverse. On the one hand, cerebral insulin may improve cognitive performance, for example, by facilitating long-term potentiation, the molecular basis of learning and memory, by modulating neurotransmitter release, or by increasing glucose availability in the brain. ${ }^{28}$ On the other hand, insulin is believed to interact with the metabolism of amyloid- $\beta$ and tau, ${ }^{28}$ key proteins in the pathogenesis of Alzheimer's disease. 
While experimental studies have identified multiple pathways through which hyperglycaemia and insulin resistance may impact cognitive performance, no studies to date have evaluated whether the effects of diabetes on cognitive performance are dependent of the degree of hyperglycaemia and/or insulin resistance. Such knowledge is, however, crucial to elucidate the aetiology of diabetes-associated cognitive problems, and hence essential for the development of strategies to prevent diabetes-associated decrements in cognitive performance.

Is there reason to believe that vascular function plays a role in diabetes-associated cognitive problems?

Type 2 diabetes is often accompanied by comorbidities such as hypertension, ${ }^{29}$ obesity, ${ }^{29}$ and hyperlipidaemia. ${ }^{30}$ Each of these traditional vascular risk factors has been associated with cognitive impairment ${ }^{31}$ and dementia, ${ }^{32}$ probably through the accumulation of vascular brain damage. ${ }^{33}$ Although type 2 diabetes is known to increase the burden of cerebral small vessel disease ${ }^{34}$ and autopsy studies keep pointing to vascular damage as a major cause of the increased risk of dementia in people with diabetes, ${ }^{35}$ epidemiological studies $^{36-41}$ have revealed conflicting results as to whether the effects of diabetes on cognitive performance are dependent of traditional vascular risk factors. In addition, reports that include mediation analyses are scarce. ${ }^{42}$ This let us to also consider the importance of vascular function.

A growing body of evidence suggests that diabetes accelerates arterial stiffening. ${ }^{43,} 44$ Stiffening of the larger elastic arteries, caused by structural and cellular changes within the arterial wall, ${ }^{45}$ may impact their cushioning function, that is, their ability to smooth out the pulsatile flow of blood coming from the intermittent contracting heart. ${ }^{43}$ As a result, arterial stiffening increases the pulsatile load on the brain, which, in turn, may cause cerebral small vessel disease that ultimately impacts cognitive performance. ${ }^{46}$, 47 Indeed, a recent meta-analysis provided some evidence linking arterial stiffness to cognitive decline and dementia risk. ${ }^{48}$ Interestingly, it has been suggested that the ability of cerebral arteries to withstand increased pulsatile pressure is reduced in the presence of diabetes, ${ }^{47}$ potentially because of the increased microvascular flow (ie, capillary hyperperfusion) that is seen in the early stages of the disease. ${ }^{49}$ This supports our hypothesis that arterial stiffness is involved in diabetes-associated cognitive decrements, in line with findings linking arterial stiffness to diabetic nephropathy ${ }^{50}$ and retinopathy. ${ }^{51}$

Research in recent years has also provided some evidence ${ }^{52,53}$ that diabetes affects the remodelling of the carotid arteries, the main suppliers of blood to the brain. Arterial remodelling describes the process of structural changes that arteries undergo with ageing ${ }^{54}$ and in response to haemodynamic and atherosclerotic stimuli, ${ }^{53}$ and is 
characterised by diameter widening and wall thickening. ${ }^{53,55,56}$ Remodelling of arteries is believed to be a compensatory mechanism to normalise circumferential wall stress, ${ }^{53}$ but it is not uniformly successful in achieving this goal. Maladaptive remodelling at the level of the carotid artery, as, for example, observed in type 2 diabetes, ${ }^{53}$ results in greater circumferential wall stress, which has been linked to cerebral small vessel disease, ${ }^{57}$, 58 and hence may affect cognitive performance. Evidence of an association between greater circumferential wall stress and impairments in cognitive performance is currently lacking, but would be a first step towards answering the question whether maladaptive remodelling is a potential mediator of diabetes-associated cognitive problems.

\section{Cognitive performance among those with type 2 diabetes}

Evidently, not everyone with diabetes will experience cognitive problems. ${ }^{59}$ In addition, individuals who do suffer from diabetes-associates cognitive decrements do not necessarily develop frank dementia. ${ }^{14}$ In view of the so-called diabetes epidemic, ${ }^{9,60}$ which is being propelled by climbing obesity rates ${ }^{61}$ and increasing life expectancy of individuals with type 2 diabetes, ${ }^{62}$ there is an urgent need to identify factors that contribute to the severity and/or progression of diabetes-associated cognitive problems. Previous clinical and epidemiological studies that confirmed measures of hyperglycaemia and insulin resistance to be associated with worse cognitive performance were commonly conducted in the general population $\left(\mathrm{eg},{ }^{63-68}\right.$ ) or in individuals without diabetes $\left(\mathrm{eg},{ }^{69-75}\right)$. Whether hyperglycaemia and insulin resistance are related to cognitive performance among individuals with type 2 diabetes remains a matter of debate. If this were the case, these metabolic factors would create potential targets for the amelioration of cognitive problems in diabetes.

\section{Aims and outline of this thesis}

The present thesis aims to elucidate whether measures of glucose metabolism and vascular function explain differences in cognitive performance between individuals with and without type 2 diabetes. In addition, we aimed to enhance our knowledge of the effects of abnormalities in glucose metabolism on cognitive performance within individuals with type 2 diabetes. As such, the present thesis may eventually help in the development of effective primary and/or secondary prevention strategies for (diabetesassociated) cognitive problems, thereby reducing the individual and societal burden of these problems.

Throughout the thesis, we used data from two cohort studies: the Maastricht Study and the Pearl Neurodegenerative Diseases. Details on these cohorts have been published previously. ${ }^{76,77}$ 
In Chapter 2, we examined, within The Maastricht Study, the mediating effects of hyperglycaemia, insulin resistance, and blood pressure-related variables on diabetesassociated differences in cognitive performance.

In Chapter 3, we assessed, within The Maastricht Study, the interplay between type 2 diabetes, arterial stiffness, and cognitive performance.

In Chapter 4, we investigated, within The Maastricht Study, the association between circumferential wall stress at the level of the carotid artery and cognitive performance. In addition, we assessed whether any such association differed between individuals with and without type 2 diabetes.

In Chapter 5, we conducted a systematic review of the literature on the association between glucose regulation (ie, glycaemia, hypoglycaemic events, insulin (resistance), and glucose-lowering treatment) and cognitive performance and abnormalities on brain magnetic resonance imaging in people with type 2 diabetes.

In Chapter 6, we evaluated, within The Maastricht Study, whether insulin-related variables (ie, fasting insulin, C-peptide and the homeostasis model assessment of insulin resistance) contribute to the variance in cognitive performance among individuals with type 2 diabetes.

As outlined earlier in this General Introduction, multiple pathways have been described that link abnormal insulin signalling to Alzheimer's disease, ${ }^{28}$ including its interference with the metabolism of amyloid- $\beta$ and tau. Insulin signalling in the human brain cannot be measured in vivo. Alternatively, insulin levels within the cerebrospinal fluid that surrounds the brain and spinal cord are the best possible reflections of insulin levels within the central nervous system, and can therefore be used to assess the cerebral effects of insulin. In Chapter 7, we investigated, with use of data from The Pearl Neurodegenerative Diseases, the association of cerebrospinal fluid insulin levels with cognitive performance and cerebrospinal fluid biomarkers of Alzheimer's disease (ie, cerebrospinal fluid amyloid- $\beta$ and tau levels).

Chapter $\mathbf{8}$ discusses the key findings of this thesis and tries to put these findings into a broader perspective. It also sheds lights on the methodological limitations that should be kept in mind when reading this thesis, and provides directions for further research. 


\section{References}

1. Oeppen, J. and J.W. Vaupel, Demography. Broken limits to life expectancy. Science, 2002. 296(5570): p. 1029-31.

2. Nationaal Kompas. Levensverwachting: achtergrondcijfers. 2012. Available from: http:// www.nationaalkompas.nl/gezondheid-en-ziekte/sterfte-levensverwachting-en-daly-s/ levensverwachting/wat-is-in-nederland-de-levensverwachting/. Last accessed February 12, 2016.

3. Newton, J.N., Future inequalities in life expectancy in England and Wales. Lancet, 2015. 386(9989): p. 115-7.

4. Bishop, N.A., T. Lu, and B.A. Yankner, Neural mechanisms of ageing and cognitive decline. Nature, 2010. 464(7288): p. 529-35.

5. Biessels, G.J., Een blijvende herinnering; oratie uitgesproken bij de aanvaarding van het ambt van hoogleraar in de Neurologie. 2013: Faculteit der Geneeskunde aan de Universiteit Utrecht.

6. Deary, I.J., et al., Age-associated cognitive decline. Br Med Bull, 2009. 92: p. 135-52.

7. Elliott, R., Executive functions and their disorders. Br Med Bull, 2003. 65: p. 49-59.

8. Koekkoek, P.S., et al., Cognitive function in patients with diabetes mellitus: guidance for daily care. Lancet Neurol, 2015. 14(3): p. 329-40.

9. Nationaal Kompas. Diabetes mellitus: incidentie, prevalentie en sterfte naar leeftijd en geslacht. 2011. Available from: http://www.nationaalkompas.nl/gezondheid-en-ziekte/ziekten-enaandoeningen/endocriene-voedings-en-stofwisselingsziekten-en-immuniteitsstoornissen/ diabetes-mellitus/omvang/. Last accessed February 12, 2016.

10. Alzheimer's Disease International. World Alzheimer Report 2015; The Global Impact of Dementia: an analysis of prevalence, incidence, cost and trends. 2015. Available from: http:// www.alz.co.uk/research/world-report-2015. Last accessed February 12, 2016.

11. Alzheimer's Australia NSW. Addressing the Stigma associated with Dementia. 2010. Available at: https://nsw.fightdementia.org.au/sites/default/files/2010NSWAddressingStigmaDiscussionPaper2.pdf. Last accessed July 23, 2015.

12. Hurd, M.D., et al., Monetary costs of dementia in the United States. N Engl J Med, 2013. 368(14): p. 1326-34.

13. Miles, W.E. and H.F. Root, Psychologic tests applied to diabetic patients. Arch Intern Med, 1922. 30(6): p. 767-777.

14. Biessels, G.J., et al., Dementia and cognitive decline in type 2 diabetes and prediabetic stages: towards targeted interventions. Lancet Diabetes Endocrinol, 2014. 2(3): p. 246-55.

15. Cheng, G., et al., Diabetes as a risk factor for dementia and mild cognitive impairment: a meta-analysis of longitudinal studies. Intern Med J, 2012. 42(5): p. 484-91.

16. Biessels, G.J., I.J. Deary, and C.M. Ryan, Cognition and diabetes: a lifespan perspective. Lancet Neurol, 2008. 7(2): p. 184-90.

17. Strachan, M.W. and J.F. Price, Diabetes. Cognitive decline and T2DM--a disconnect in the evidence? Nat Rev Endocrinol, 2014. 10(5): p. 258-60.

18. Crane, P.K., et al., Glucose levels and risk of dementia. N Engl J Med, 2013. 369(6): p. 540-8.

19. Tan, Z.S., et al., Association of metabolic dysregulation with volumetric brain magnetic resonance imaging and cognitive markers of subclinical brain aging in middle-aged adults: the Framingham Offspring Study. Diabetes Care, 2011. 34(8): p. 1766-70.

20. Kilander, L., et al., Hypertension is related to cognitive impairment: a 20-year follow-up of 999 men. Hypertension, 1998. 31(3): p. 780-6.

21. Kahn, S.E., M.E. Cooper, and S. Del Prato, Pathophysiology and treatment of type 2 diabetes: perspectives on the past, present, and future. Lancet, 2014. 383(9922): p. 1068-83.

22. Brownlee, M., Biochemistry and molecular cell biology of diabetic complications. Nature, 2001. 414(6865): p. 813-20. 
23. Biessels, G.J., et al., Risk of dementia in diabetes mellitus: a systematic review. Lancet Neurol, 2006. 5(1): p. 64-74.

24. Gispen, W.H. and G.J. Biessels, Cognition and synaptic plasticity in diabetes mellitus. Trends Neurosci, 2000. 23(11): p. 542-9.

25. Sima, A.A., H. Kamiya, and Z.G. Li, Insulin, C-peptide, hyperglycemia, and central nervous system complications in diabetes. Eur J Pharmacol, 2004. 490(1-3): p. 187-97.

26. Mooradian, A.D., Central nervous system complications of diabetes mellitus--a perspective from the blood-brain barrier. Brain Res Brain Res Rev, 1997. 23(3): p. 210-8.

27. Last, D., et al., Global and regional effects of type 2 diabetes on brain tissue volumes and cerebral vasoreactivity. Diabetes Care, 2007. 30(5): p. 1193-9.

28. Cholerton, B., L.D. Baker, and S. Craft, Insulin, cognition, and dementia. Eur J Pharmacol, 2013. 719(1-3): p. 170-9.

29. Colosia, A.D., R. Palencia, and S. Khan, Prevalence of hypertension and obesity in patients with type 2 diabetes mellitus in observational studies: a systematic literature review. Diabetes Metab Syndr Obes, 2013. 6: p. 327-38.

30. Mooradian, A.D., Dyslipidemia in type 2 diabetes mellitus. Nat Clin Pract Endocrinol Metab, 2009. 5(3): p. 150-9.

31. Van den Berg, E., et al., Type 2 diabetes mellitus, hypertension, dyslipidemia and obesity: A systematic comparison of their impact on cognition. Biochim Biophys Acta, 2009. 1792(5): p. 470-81.

32. Kloppenborg, R.P., et al., Diabetes and other vascular risk factors for dementia: which factor matters most? A systematic review. Eur J Pharmacol, 2008. 585(1): p. 97-108.

33. Kennelly, S.P., B.A. Lawlor, and R.A. Kenny, Blood pressure and dementia - a comprehensive review. Ther Adv Neurol Disord, 2009. 2(4): p. 241-60.

34. Biessels, G.J. and Y.D. Reijmer, Brain changes underlying cognitive dysfunction in diabetes: what can we learn from MRI? Diabetes, 2014. 63(7): p. 2244-52.

35. Ahtiluoto, S., et al., Diabetes, Alzheimer disease, and vascular dementia: a population-based neuropathologic study. Neurology, 2010. 75(13): p. 1195-202.

36. Peila, R., et al., Type 2 diabetes, APOE gene, and the risk for dementia and related pathologies: The Honolulu-Asia Aging Study. Diabetes, 2002. 51(4): p. 1256-62.

37. Macknight, C., et al., Diabetes mellitus and the risk of dementia, Alzheimer's disease and vascular cognitive impairment in the Canadian Study of Health and Aging. Dement Geriatr Cogn Disord, 2002. 14(2): p. 77-83.

38. Fontbonne, A., et al., Changes in cognitive abilities over a 4-year period are unfavorably affected in elderly diabetic subjects: results of the Epidemiology of Vascular Aging Study. Diabetes Care, 2001. 24(2): p. 366-70.

39. Kanaya, A.M., et al., Change in cognitive function by glucose tolerance status in older adults: a 4-year prospective study of the Rancho Bernardo study cohort. Arch Intern Med, 2004. 164(12): p. 1327-33.

40. Asimakopoulou, K.G., S.E. Hampson, and N.J. Morrish, Neuropsychological functioning in older people with type 2 diabetes: the effect of controlling for confounding factors. Diabet Med, 2002. 19(4): p. 311-6.

41. Gregg, E.W., et al., Is diabetes associated with cognitive impairment and cognitive decline among older women? Study of Osteoporotic Fractures Research Group. Arch Intern Med, 2000. 160(2): p. 174-80.

42. McFall, G.P., et al., Testing covariates of Type 2 diabetes-cognition associations in older adults: moderating or mediating effects? Neuropsychology, 2010. 24(5): p. 547-62.

43. Stehouwer, C.D., R.M. Henry, and I. Ferreira, Arterial stiffness in diabetes and the metabolic syndrome: a pathway to cardiovascular disease. Diabetologia, 2008. 51(4): p. 527-39.

44. Henry, R.M., et al., Arterial stiffness increases with deteriorating glucose tolerance status: the Hoorn Study. Circulation, 2003. 107(16): p. 2089-95. 
45. Zieman, S.J., V. Melenovsky, and D.A. Kass, Mechanisms, pathophysiology, and therapy of arterial stiffness. Arterioscler Thromb Vasc Biol, 2005. 25(5): p. 932-43.

46. Mitchell, G.F., et al., Arterial stiffness, pressure and flow pulsatility and brain structure and function: the Age, Gene/Environment Susceptibility--Reykjavik study. Brain, 2011. 134(Pt 11): p. 3398-407.

47. O'Rourke, M.F. and M.E. Safar, Relationship between aortic stiffening and microvascular disease in brain and kidney: cause and logic of therapy. Hypertension, 2005. 46(1): p. 200-4.

48. Van Sloten, T.T., et al., Association between arterial stiffness, cerebral small vessel disease and cognitive impairment: A systematic review and meta-analysis. Neurosci Biobehav Rev, 2015. 53: p. 121-30.

49. Tooke, J.E., Microvascular function in human diabetes. A physiological perspective. Diabetes, 1995. 44(7): p. 721-6.

50. Kimoto, E., et al., Regional arterial stiffness in patients with type 2 diabetes and chronic kidney disease. J Am Soc Nephrol, 2006. 17(8): p. 2245-52.

51. Rema, M., et al., Association of carotid intima-media thickness and arterial stiffness with diabetic retinopathy: the Chennai Urban Rural Epidemiology Study (CURES-2). Diabetes Care, 2004. 27(8): p. 1962-7.

52. Kozakova, M., et al., Glucose-related arterial stiffness and carotid artery remodeling: a study in normal subjects and type 2 diabetes patients. J Clin Endocrinol Metab, 2014. 99(11): p. E2362-6.

53. Henry, R.M., et al., Carotid arterial remodeling: a maladaptive phenomenon in type 2 diabetes but not in impaired glucose metabolism: the Hoorn study. Stroke, 2004. 35(3): p. 671-6.

54. Lakatta, Eg, and D. Levy, Arterial and cardiac aging: major shareholders in cardiovascular disease enterprises: Part I: aging arteries: a "set up" for vascular disease. Circulation, 2003. 107(1): p. 139-46.

55. Ward, M.R., et al., Arterial remodeling. Mechanisms and clinical implications. Circulation, 2000. 102(10): p. 1186-91.

56. Pasterkamp, G., P.F. Fitzgerald, and D.P. de Kleijn, Atherosclerotic expansive remodeled plaques: a wolf in sheep's clothing. J Vasc Res, 2002. 39(6): p. 514-23.

57. Okada, Y., et al., Mechanical stresses, arterial stiffness, and brain small vessel diseases: Shimanami Health Promoting Program Study. Stroke, 2014. 45(11): p. 3287-92.

58. Brisset, M., et al., Large-vessel correlates of cerebral small-vessel disease. Neurology, 2013. 80(7): p. 662-9.

59. Van den Berg, E., Y.D. Reijmer, and G.J. Biessels, Diabetes and the Brain; Cognition in Type 2 Dlabetes or Pre-diabetic stages. 1st ed. Comptempary Diabetes, ed. A. Veves. 2010, New York, NY: Humana Press.

60. Mokdad, A.H., et al., Diabetes trends in the U.S.: 1990-1998. Diabetes Care, 2000. 23(9): p. 1278-83.

61. Visscher, T.L., D. Kromhout, and J.C. Seidell, Long-term and recent time trends in the prevalence of obesity among Dutch men and women. Int J Obes Relat Metab Disord, 2002. 26(9): p. 1218-24.

62. Ioacara, S., et al., Improvements in life expectancy in adult type 2 diabetes patients in the last six decades. Diabetes Res Clin Pract, 2011. 92(3): p. 400-4.

63. Kalmijn, S., et al., Glucose intolerance, hyperinsulinaemia and cognitive function in a general population of elderly men. Diabetologia, 1995. 38(9): p. 1096-102.

64. Weinstein, G., et al., Glucose indices are associated with cognitive and structural brain measures in young adults. Neurology, 2015. 84(23): p. 2329-37.

65. Stolk, R.P., et al., Insulin and cognitive function in an elderly population. The Rotterdam Study. Diabetes Care, 1997. 20(5): p. 792-5.

66. Luchsinger, J.A., et al., Hyperinsulinemia and risk of Alzheimer disease. Neurology, 2004. 63(7): p. 1187-92. 
67. Benedict, C., et al., Impaired insulin sensitivity as indexed by the HOMA score is associated with deficits in verbal fluency and temporal lobe gray matter volume in the elderly. Diabetes Care, 2012. 35(3): p. 488-94.

68. Kuusisto, J., et al., Association between features of the insulin resistance syndrome and Alzheimer's disease independently of apolipoprotein E4 phenotype: cross sectional population based study. BMJ, 1997. 315(7115): p. 1045-9.

69. Di Bonito, P., et al., Impact of impaired fasting glucose and other metabolic factors on cognitive function in elderly people. Nutr Metab Cardiovasc Dis, 2007. 17(3): p. 203-8.

70. Sanz, C.M., et al., Relationship between markers of insulin resistance, markers of adiposity, $\mathrm{HbA1c}$, and cognitive functions in a middle-aged population-based sample: the MONA LISA study. Diabetes Care, 2013. 36(6): p. 1512-21.

71. Carantoni, M., et al., Alzheimer disease and vascular dementia: relationships with fasting glucose and insulin levels. Dement Geriatr Cogn Disord, 2000. 11(3): p. 176-80.

72. Backestrom, A., et al., Glucose but not insulin or insulin resistance is associated with memory performance in middle-aged non-diabetic women: a cross sectional study. Diabetol Metab Syndr, 2015. 7: p. 20.

73. Kerti, L., et al., Higher glucose levels associated with lower memory and reduced hippocampal microstructure. Neurology, 2013. 81(20): p. 1746-52.

74. Young, S.E., A.G. Mainous, 3rd, and M. Carnemolla, Hyperinsulinemia and cognitive decline in a middle-aged cohort. Diabetes Care, 2006. 29(12): p. 2688-93.

75. Okereke, O.I., et al., Fasting plasma insulin, C-peptide and cognitive change in older men without diabetes: results from the Physicians' Health Study II. Neuroepidemiology, 2010. 34(4): p. 200-7.

76. Schram, M.T., et al., The Maastricht Study: an extensive phenotyping study on determinants of type 2 diabetes, its complications and its comorbidities. Eur J Epidemiol, 2014. 29(6): p. 439-51.

77. Aalten, P., et al., The Dutch Parelsnoer Institute--Neurodegenerative diseases; methods, design and baseline results. BMC Neurol, 2014. 14: p. 254. 
General introduction 


\section{Chapter 2}

\section{THE ROLE OF HYPERGLYCAEMIA, INSULIN RESISTANCE, AND BLOOD PRESSURE IN DIABETES-ASSOCIATED DIFFERENCES IN COGNITIVE PERFORMANCE: THE MAASTRICHT STUDY}

Stefan L.C. Geijselaers, Simone J.S. Sep, Danny Claessens, Miranda T. Schram, Martin P.J. van Boxtel, Ronald M.A. Henry, Frans R.J. Verhey, Abraham A. Kroon, Pieter C. Dagnelie, Casper G. Schalkwijk, Carla J.H. van der Kallen, Geert Jan Biessels, Coen D.A. Stehouwer 


\begin{abstract}
Background

Although type 2 diabetes is well known to be associated with decrements in cognitive performance, the underlying mechanisms remain poorly understood. We studied to what extent differences in cognitive performance between individuals with different glucose metabolism status are potentially attributable to hyperglycaemia, insulin resistance, and blood pressure-related variables.
\end{abstract}

\title{
Methods
}

We used cross-sectional data of 2531 participants from The Maastricht Study (60 \pm 8 years, 52\% men, $n=666$ with type 2 diabetes), all of whom completed a neuropsychological test battery. Hyperglycaemia was assessed by a composite index of fasting glucose, post-load glucose, glycated haemoglobin $(\mathrm{HbA1C})$, and skin autofluorescence; insulin resistance by the homeostasis model assessment index (HOMA-IR); and blood pressure-related variables included 24-hour ambulatory pressures, their weighted standard deviations, and the use of antihypertensive medication. Linear regression analyses were used to estimate the mediating effects of these factors on differences in cognitive performance.

\section{Findings}

After adjustment for age, sex, and education, individuals with type 2 diabetes (HbA1c $6.8 \pm 1.0 \%$, HOMA-IR 1.7 [1.1-2.6], blood pressure $122 \pm 12 / 73 \pm 7 \mathrm{mmHg}$ ), compared to those with normal glucose metabolism (HbA1c 5.5 $\pm 0.3 \%$, HOMA-IR 1.0 [0.7-1.4], blood pressure $117 \pm 11 / 73 \pm 7$ $\mathrm{mmHg}$ ), performed worse in all cognitive domains (mean differences in domain scores: memory -0.087 , processing speed -0.196 , executive function and attention $-0.182 ; p$-values $<0.032$ ), whereas individuals with prediabetes did not. Diabetes-associated differences in processing speed and executive function and attention were largely explained by hyperglycaemia (mediating effect [bootstrapped 95\% confidence interval] 79.6\% [36.6;123.4\%] and 50.3\% [0.6;101.2\%], respectively), and, for processing speed, to a lesser extent by blood pressure-related variables $(17.7 \%[5.6 ; 30.1 \%])$, but not by insulin resistance. None of the factors explained the differences in memory function.

\section{Interpretation}

Our cross-sectional data suggest that early glycaemic and blood pressure control may be promising therapeutic targets for the prevention of diabetes-associated decrements in cognitive performance. 


\section{Introduction}

Almost a century ago, Miles and Root were the first to report a link between diabetes and cognitive performance. ${ }^{1}$ Since then, it has become increasingly evident that type 2 diabetes, the most common form of diabetes, as well as its precursor, prediabetes, are associated with a variety of cognitive changes. ${ }^{2}$ These not only include subtle cognitive decrements, but also mild cognitive impairment and dementia. ${ }^{2}$ Over the coming decades, the prevalence of these diabetes-associated cognitive problems is expected to rise dramatically as a result of the ongoing diabetes epidemic and the increasing life expectancy of individuals with diabetes, ${ }^{3}$ which highlights the need to develop preventive interventions. Yet, the mechanisms underlying diabetes-associated cognitive problems remain elusive. Observational data, however, suggest that both metabolic and vascular factors are likely to be involved. ${ }^{2,4}$

Insulin resistance and progressive $\beta$-cell dysfunction are the hallmarks of type 2 diabetes, leading to hyperglycaemia and abnormal insulin signalling, which may directly affect the brain. Hyperglycaemia is known to increase oxidative stress through mitochondrial overproduction of superoxide, ${ }^{5}$ which, in turn, can trigger neuronal dysfunction and cell death. ${ }^{6}$ In addition, chronic hyperglycaemia has been related to blood-brain barrier dysfunction and reduced cerebral blood flow. ${ }^{2}$ Abnormal insulin signalling may impact cognitive performance by affecting long-term potentiation, modulating neurotransmitter release, and interfering with the metabolism of amyloid- $\beta$ and tau, key proteins in the pathogenesis of Alzheimer's disease. ${ }^{7}$

Type 2 diabetes typically develops in the context of an adverse cardiovascular risk profile with obesity, hypertension, and dyslipidaemia. Importantly, there is increasing evidence that each of these comorbid vascular risk factors is also linked to cognitive impairment ${ }^{8}$ and dementia, ${ }^{9}$ with hypertension being the risk factor most consistently associated with cognitive performance ${ }^{8,9}$ through mechanisms of vascular brain damage. ${ }^{10}$ As such, hypertension, present in up to $75 \%$ of the individuals with type 2 diabetes, ${ }^{11}$ may contribute, either alone or in concert with metabolic factors, to the cognitive decrements seen in diabetes.

To date, numerous studies (eg, ${ }^{12-14}$ ) have confirmed that markers of hyperglycaemia, insulin resistance, and abnormalities in blood pressure are associated with cognitive performance. In general, however, little is known about the complex interrelationship among these factors and it remains unclear to what extent metabolic and vascular factors also mediate, and thus explain, diabetes-associated cognitive decrements. Therefore, the present study aimed to examine to what extent differences in cognitive 
performance between individuals with type 2 diabetes or prediabetes on the one hand, and those with a normal glucose metabolism (NGM) on the other are attributable to hyperglycaemia, insulin resistance, and blood pressure-related variables.

\section{Methods}

\section{Study population}

The present analysis was conducted with data of The Maastricht Study, an ongoing observational prospective population-based cohort study, whose rationale and design have been described previously ${ }^{15}$ and are outlined in the Supplement. For the present study, cross-sectional data from the first 3451 participants were used, who completed the baseline survey between November 2010 and September 2013. We excluded participants with types of diabetes other than type 2 ( $n=41$, of whom 37 had type 1 diabetes). Each participant provided written informed consent and completed all examinations within a time window of three months. The Maastricht Study was approved by the institutional medical ethical committee (NL31329.068.10) and the Minister of Health, Welfare and Sports of the Netherlands, on the basis of the Health Council's opinion (Permit 131088105234-PG).

\section{Glucose metabolism status}

A standardised two-hour seven-sample oral glucose tolerance test (OGTT) was used to determine the participants' glucose metabolism status, as described elsewhere ${ }^{15}$ and detailed in the Supplement.

\section{Cognitive performance}

All individuals completed a concise (30 $\mathrm{min}$ ) neuropsychological test battery to assess cognitive performance. ${ }^{15}$ For conceptual clarity, test scores were standardised and divided into three cognitive domains (ie, memory function, executive function and attention, and information processing speed), as detailed previously ${ }^{16}$ and summarised in the Supplement.

\section{Hyperglycaemia, insulin resistance, and blood pressure-related variables}

For reasons of statistical efficiency, we constructed a composite index of hyperglycaemia which included fasting and post-load plasma glucose levels, as measures of short-term hyperglycaemia, and glycated haemoglobin (HbA1c) and skin autofluorescence (SAF), as representatives of long-term hyperglycaemia. To this end, individual measures were standardised into z-scores and averaged. Note that individuals who used insulin had no data on post-load glucose as they were excluded from the OGTT, and, hence, their

composite index of hyperglycaemia consisted of fasting glucose, HbA1c, and SAF. Details 
on the measurements of the individual components are provided in the Supplement. For sensitivity purposes, we also constructed a composite index that focused on longterm hyperglycaemia, as fasting and post-load glucose levels were directly used for the classification of glucose metabolism status.

The homeostasis model assessment of insulin resistance (HOMA-IR), calculated from fasting insulin and glucose levels using the HOMA-calculator version 2.2.3 for Windows (available from https://www.dtu.ox.ac.uk/ homacalculator), was used as an index of insulin resistance. HOMA-IR is a widely used surrogate marker of insulin resistance and corresponds reasonably well to clamp-derived measures of insulin sensitivity. ${ }^{17}$ For sensitivity purposes, we also calculated HOMA-IR with C-peptide rather than insulin levels and constructed a composite index that combined both HOMA-IR indices. Because C-peptide is not cleared by the liver, ${ }^{18}$ it is considered a more stable and thus accurate marker of endogenous insulin secretion, which potentially makes HOMA-IR calculated with C-peptide a better marker of peripheral insulin resistance. ${ }^{19}$ Fasting insulin levels and C-peptide were quantified on a Meso Scale custom duplex assay (Meso Scale Discovery, Gaithersburg, Maryland, USA).

Based on ambulatory blood pressure data (Supplement), a composite index was created that incorporated 24-hour systolic and diastolic blood pressure and their weighted standard deviations. Blood pressure variability was included as it is increasingly recognised to be associated with worse cognitive performance ${ }^{20}$ and an increased dementia risk, ${ }^{21}$ independent of actual blood pressure. For the analyses, this composite index was combined with use of antihypertensive medication ${ }^{15}$ in order to account for previous exposure to elevated blood pressure. For sensitivity analyses, we also constructed an index that included 24-hour pulse pressure rather than 24-hour systolic and diastolic blood pressure, as there is increasing evidence linking arterial stiffening to cognitive performance..$^{22}$

\section{Covariates}

Details on the assessments of the covariates are provided in the Supplement.

\section{Statistical analyses}

The age-, sex-, and education-adjusted associations of glucose metabolism status with cognitive performance were estimated by linear regression analyses with cognitive performance as the dependent variable. Individuals with NGM served as the reference group. To justify the use of composite indices, the directions of the associations between, on the one hand, the individual components of these indices, and, on the other, cognitive performance were evaluated. 
Next, we used linear regression to analyse the associations between, on the one hand, the indices used for hyperglycaemia, insulin resistance, and blood pressure, and, on the other, cognitive performance. Using multiplicative interaction terms, we also examined whether these associations differed by glucose metabolism status, with age, sex, and educational level as covariates. Next, we tested for pairwise statistical interaction between the indices used for hyperglycaemia, insulin resistance, and blood pressure (eg, hyperglycaemia*insulin resistance) with regard to their association with cognitive performance.

The extent to which hyperglycaemia, insulin resistance, and blood pressure-related variables mediated the association between glucose metabolism status and cognitive performance was then determined by adding these variables to the previously described regression models. Both independent and joint mediation effects were evaluated, and expressed as the (percentage) change of the regression coefficients of the dummies coding glucose metabolism status. Corresponding 95\% confidence interval were estimated with use of a SPSS macro provided by Preacher and Hayes (10,000 bootstrap iterations) ${ }^{23}$ We additionally analysed the mediating effects of the individual components of the composite indices. Collinearity diagnostics (ie, tolerance $<0.1$ and/or variance inflation factor $>10$ ) were used to detect multicollinearity between the independent variables.

Multiple sensitivity analyses were performed to explore the mediating effects of alternatively constructed composite indices and to evaluate the effects of additional adjustment for cardiovascular risk factors, as detailed in the Supplement. All analyses were conducted with SPSS version 22.0 for Windows (IBM SPSS, IBM Corp, Armonk, NY, USA) at a significance level of $5 \%$, except for the tests of interaction effects, where a significance level of $10 \%$ was used. No adjustments were made for multiple comparisons. ${ }^{24}$ Variables with a skewed distribution were transformed with the natural logarithm prior to analyses.

\section{Results}

\section{Study population}

From the 3410 individuals who were initially eligible for the present study, 225 (6.6\%) were excluded as they had no, or incomplete, data on cognitive performance, mostly because they were unwilling to undergo, or unmotivated to complete, the cognitive assessment. An additional 654 (19.2\%) individuals were excluded due to missing data on the composite indices of hyperglycaemia ( $n=220$, of whom $n=176$ missed data on SAF), insulin resistance $(n=46)$, and/or blood pressure abnormalities $(n=437$, of whom $n=365$ had no data on 24-hour ambulatory blood pressure), leaving 2531 participants for the 


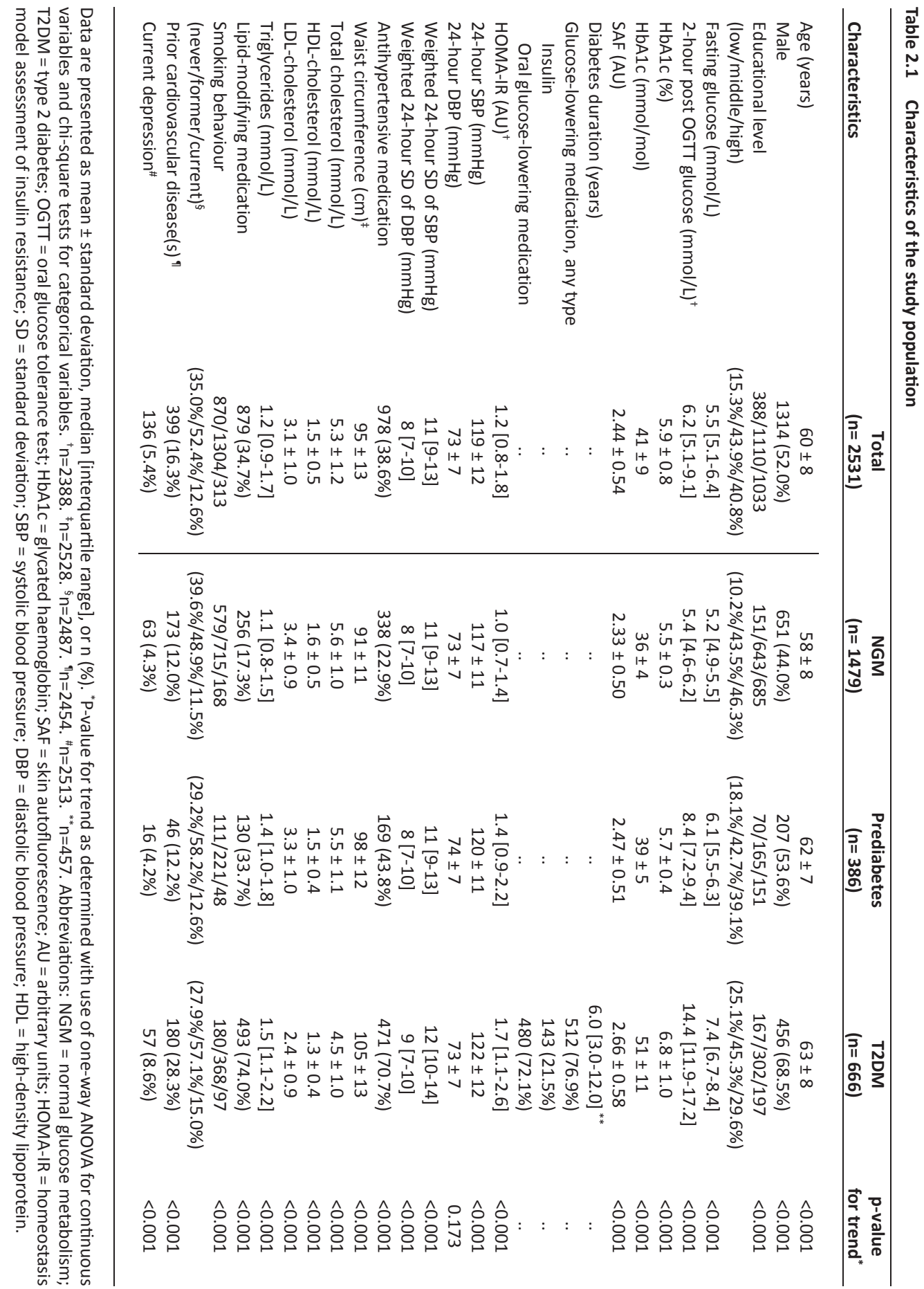


present analyses. Details on those who were excluded from the present analysis are provided in Table S2.1 (Supplement).

Demographic and clinical characteristics of the final study population, stratified by glucose metabolism status, are presented in Table 2.1. As expected, the cardiovascular risk profile worsened with deteriorating glucose metabolism status. Note, nevertheless, that individuals with diabetes were generally well controlled for their diabetes and comorbid vascular risk factors, as, for example, absolute differences in 24-hour blood pressure between individuals with diabetes and those with NGM were small (122/73 vs. $119 / 73 \mathrm{mmHg}$ ). Of the 666 individuals who were classified as having type 2 diabetes, 103 (15.5\%) were newly diagnosed at study entry.

\section{Glucose metabolism status and cognitive performance}

Figure 2.1 shows the age-, sex-, and education-adjusted mean differences in cognitive performance between individuals with different glucose metabolism status. Despite an overall trend toward lower cognitive performance with deteriorating glucose metabolism status, statistically significant worse cognitive performance was only observed in individuals with diabetes. Detailed analyses revealed that these differences were primarily driven by the relatively poor cognitive performance of individuals with previously diagnosed diabetes. The magnitude of diabetes-associated worse cognitive performance was similar across cognitive domains, except for memory function, where the magnitude was less than half of that observed for processing speed and executive function and attention.

\section{Hyperglycaemia, insulin resistance, blood pressure-related variables, and cognitive performance}

After adjustment for age, sex, and educational level, statistically significant negative associations were found between the composite index of hyperglycaemia and performance in the domains of processing speed and executive function and attention, between insulin resistance and performance in the domain of executive function and attention, and between the index of blood pressure-related variables and performance in all the cognitive domains assessed (Table S2.2, Supplement). Importantly, these associations did not differ by glucose metabolism status, but we did observe interaction between hyperglycaemia and the use of antihypertensive medication in their association with performance in the domains of memory ( $p$ for interaction $=0.009$ ) and processing speed ( $p$ for interaction $=0.035$ ). Specifically, the presence of the one tended to exacerbate the negative effects of the other (data not shown). The direction of the associations, with cognitive performance, of the individual components of the composite indices was similar to that of the respective composite index (Table S2.3, Supplement). 


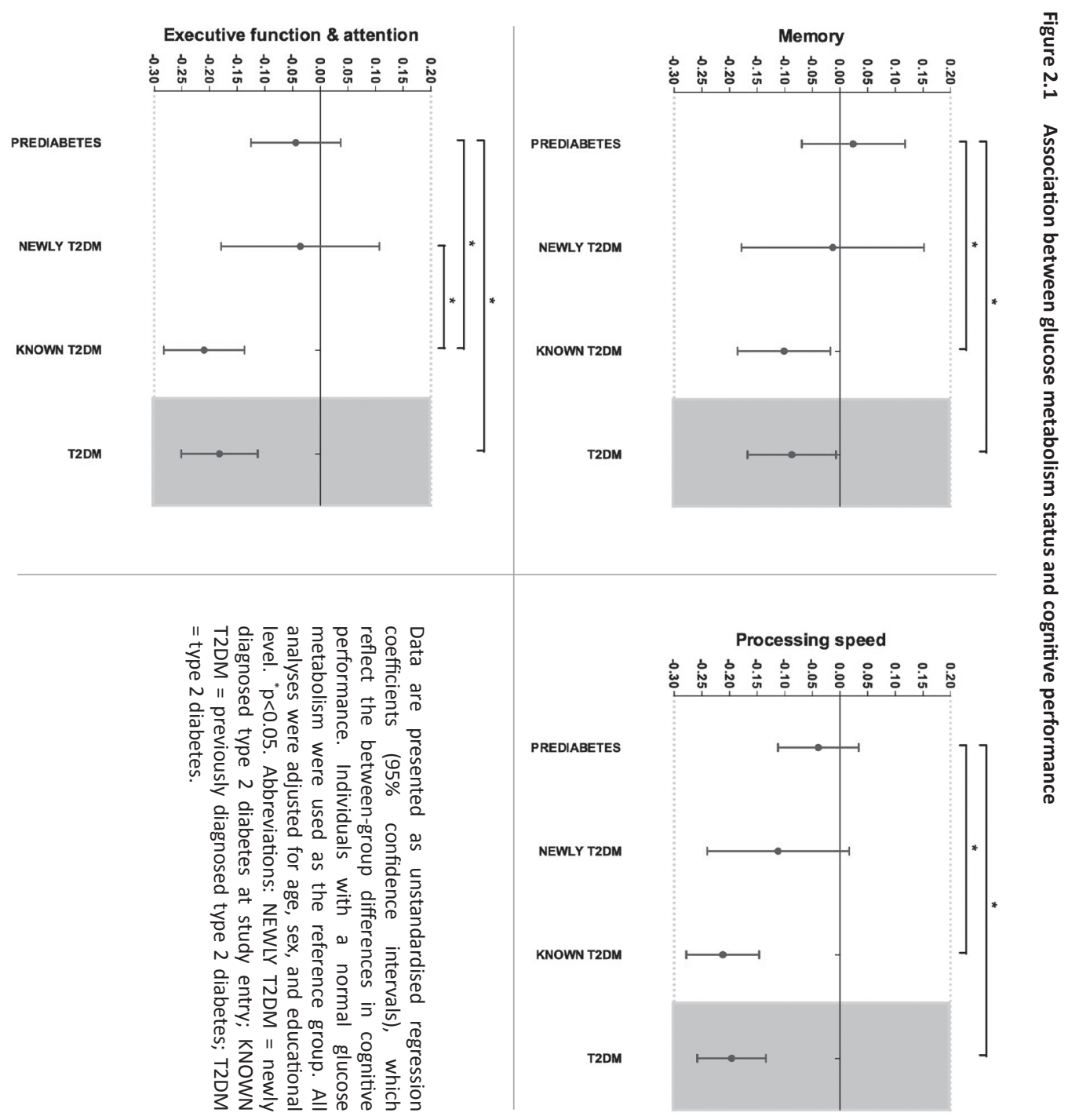



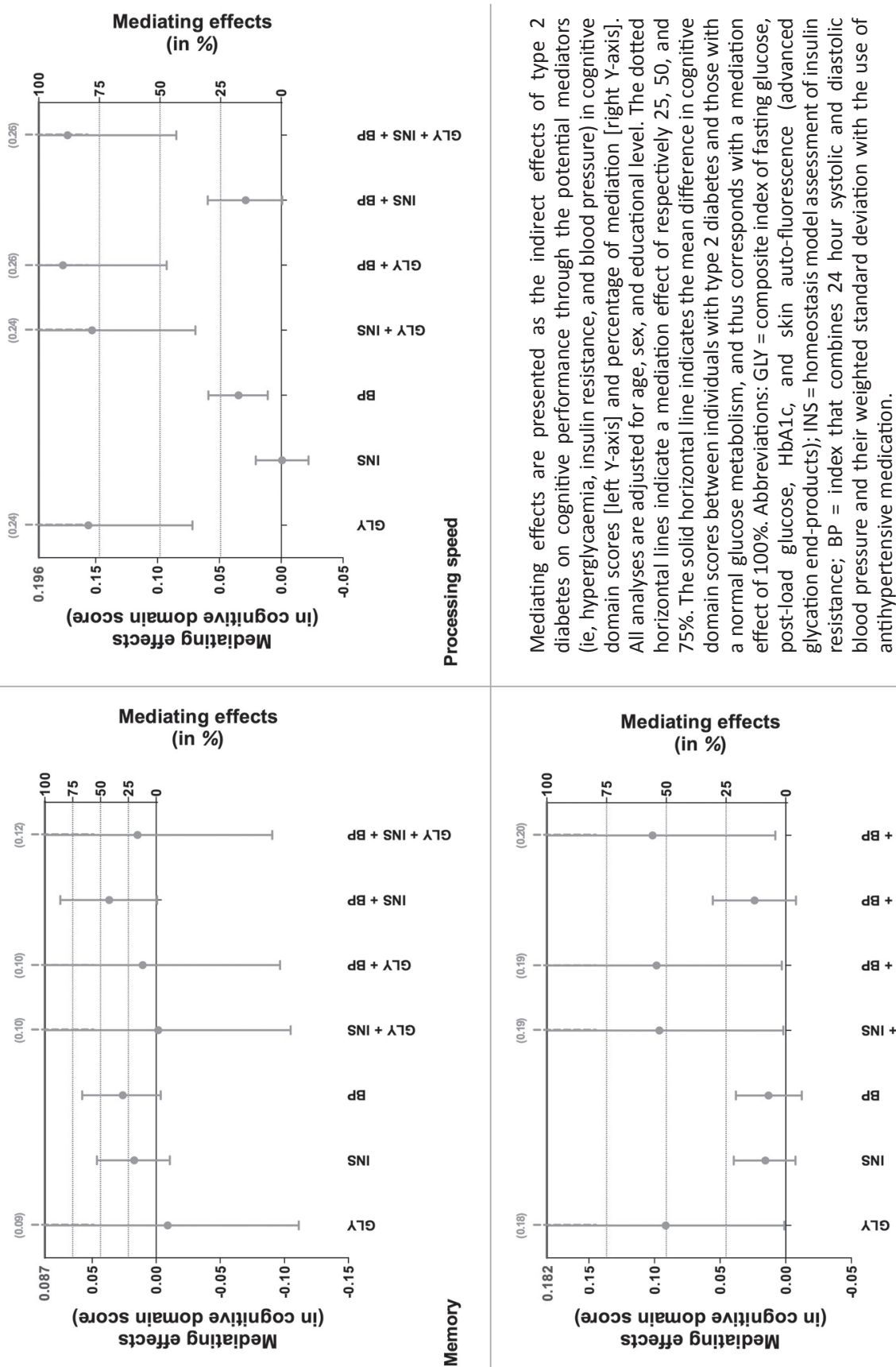

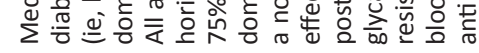

Mediating effects

(in \%)

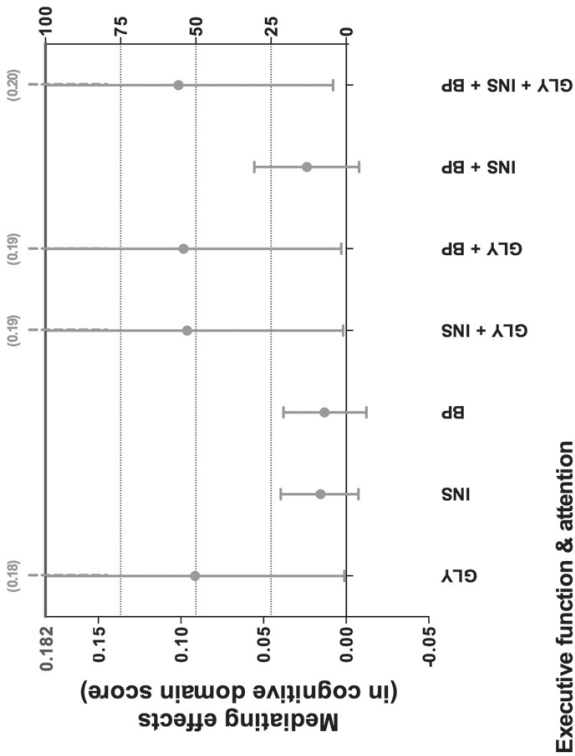




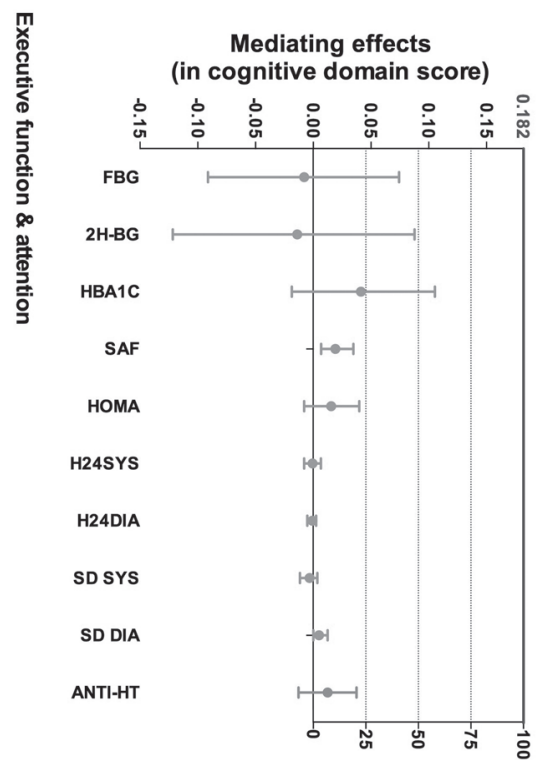

(\% u!)

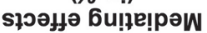

$\frac{3}{3}$

Mediating effects (in cognitive domain score)

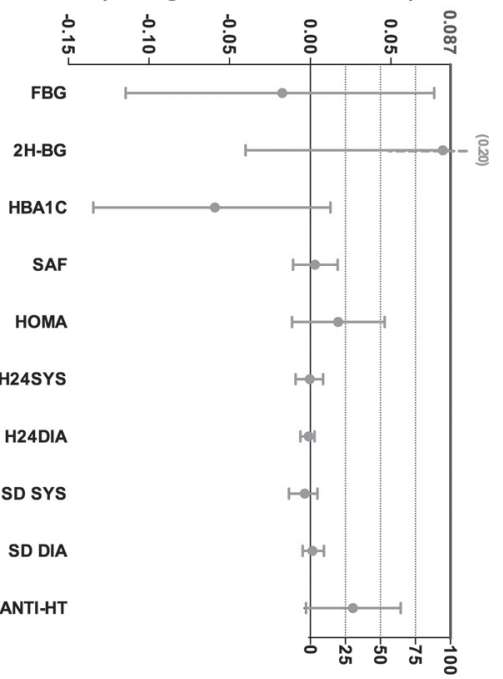

(\% u!)

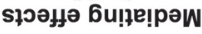

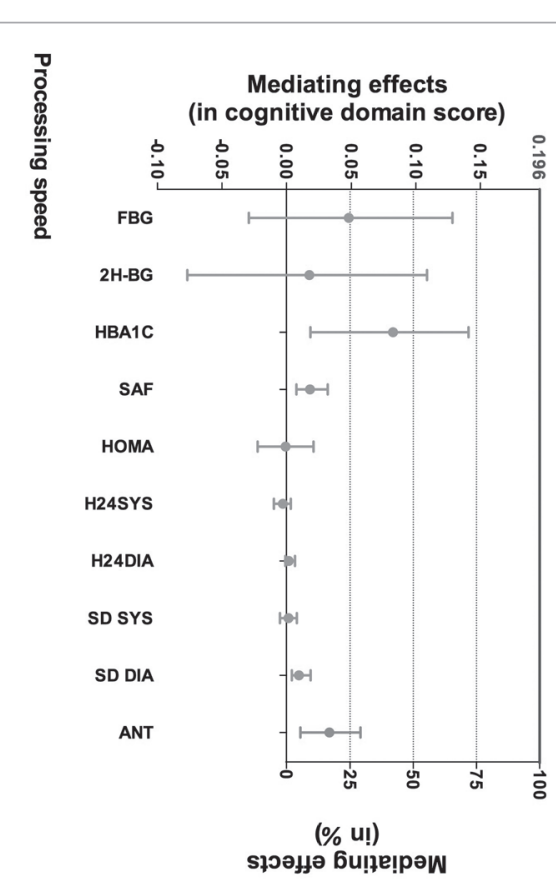

(\% u!) sџวәуә 6u!ฺe!pəw

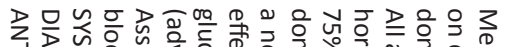

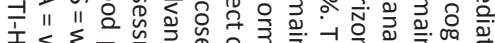

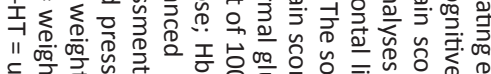

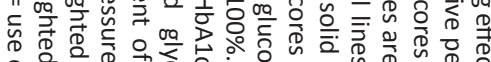

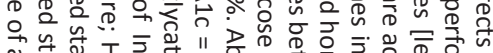

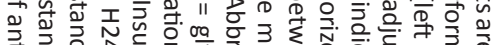

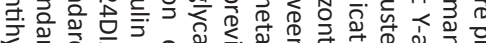

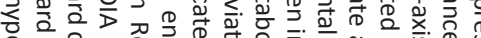

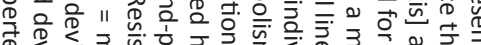

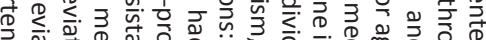

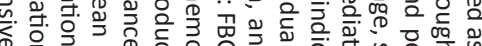

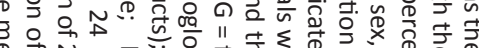

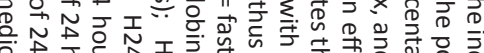

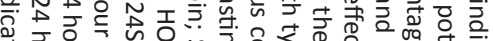

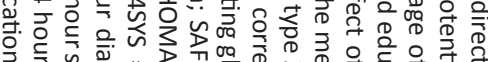
荡

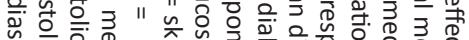

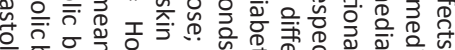

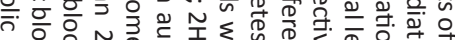

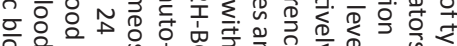

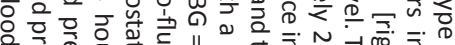

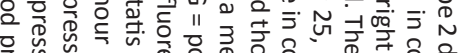

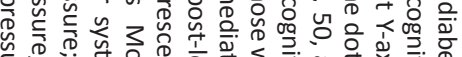

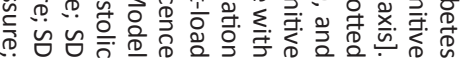

告 
Mediating effects of hyperglycaemia, insulin resistance, and blood pressure-related variables in diabetes-associated worse cognitive performance

As shown in Figure 2.2, differences in performance in the domains of processing speed and executive function and attention between individuals with type 2 diabetes and those with NGM were largely explained by hyperglycaemia, with statistically significant mediating effects of $79.6 \%$ (bootstrapped 95\% confidence interval 36.6 to $123.4 \%$ ) and $50.3 \%$ (0.6 to $101.2 \%$ ), respectively. Differences in processing speed were also partly explained by blood pressure-related variables (mediating effect $17.7 \%$ (5.6 to $30.1 \%)$ ), while insulin resistance had no mediating effects on the association of type 2 diabetes with either processing speed or executive function and attention. Consequently, any combination of the mediators resembled the mediating effects observed for hyperglycaemia and/or blood pressure. Differences in memory function were not explained by hyperglycaemia, nor by insulin resistance or blood pressure-related variables.

Evaluation of the combined mediating effects of any combination of hyperglycaemia, insulin resistance, and blood-pressure related variables (Figure 2.2) indicated that the mediating effects of hyperglycaemia and blood pressure were additive, ie, any combination of mediators resembled the summed effects of the individual mediators. At the same time, as suggested by the multiplicative interaction we observed between hyperglycaemia and the use of antihypertensive medication, the mediating effects of hyperglycaemia appeared to be more pronounced among users of antihypertensive medication compared to non-users (data not shown). Conversely, the mediating effects of blood pressure tend to increase with the severity of hyperglycaemia (data not shown).

Figure 2.3 shows the results obtained when the mediating effects of the individual components of the composite indices were considered. Again, diabetes-associated worse performance in processing speed and executive function and attention was partially explained by measures of hyperglycaemia, with $\mathrm{HbA} 1 \mathrm{c}$ being the most important mediator. Likewise, the use of antihypertensive medication and, to a much lesser extent, the weighted standard deviation of diastolic blood pressure, mediated a small part of the diabetes-associated differences in performance in processing speed and executive function and attention. For memory function, none of the individual components of any of the composite indices showed statistically significant mediating effects.

A similar pattern of mediation was observed when the mediating effects of the composite indices on the (statistically non-significant) differences in cognitive performance between individuals with NGM and prediabetes, or on the (significant) differences between prediabetes and type 2 diabetes, were explored (data not shown). Collinearity diagnostics revealed no multicollinearity in any of the mediation analyses 
(ie, all tolerance values $\geq 0.206$ and variance inflation factors $\leq 4.843$ ).

\section{Sensitivity analyses}

Qualitatively similar results to those reported here were observed in a range of sensitivity analyses (Supplement) in which we analysed individuals with newly diagnosed type 2 diabetes separately from those with a prior diagnoses of diabetes; excluded individuals treated with insulin; performed additional adjustments for prior cardiovascular disease, cardiovascular risk factors, and depression; focused on long-term hyperglycaemia; calculated HOMA-IR with fasting C-peptide instead of insulin levels; combined the HOMA-IR indices with fasting insulin and C-peptide levels; considered 24-hour pulse pressure rather than systolic and diastolic pressure; evaluated the presence of quadratric associations; and explored the effects of our complete case analysis approach by using all available data (ie, allowing composite indices to be constructed when participants had data on at least one of the individual components). Importantly, blood pressure lost its mediating effects when the use of antihypertensive medication was excluded from the index of blood pressure-related variables (Figure S2.1, Supplement).

\section{Discussion}

In the present study, diabetes-associated worse cognitive performance, except for memory function, was largely explained by hyperglycaemia and, to a lesser extent, by blood pressure-related variables, whereas insulin resistance had no mediating effects. Comparable results were obtained after adjustment for additional cardiovascular risk factors such as waist circumference and dyslipidaemia. As such, our findings indicate that it is primarily the glycaemic load itself, rather than the cardiovascular context in which diabetes typically develops, that contributes to the differences in cognitive performance between individuals with relatively well-controlled type 2 diabetes and those with NGM.

The profile of diabetes-related differences in cognitive performance observed in the present study is consistent with that of mild differences across multiple domains reported in previous systematic reviews. ${ }^{8,25}$ For memory function, however, relatively smaller differences were observed than have been previously reported, which may have an aetiological explanation, ie, that a diminished ability to efficiently and effectively process unstructured information is the primary deficit underlying diabetes-associated decrements in cognitive performance. ${ }^{26}$ In addition, individuals with diabetes in our cohort had relatively good glycaemic control, which, along with their relatively high educational level and thus cognitive reserve, ${ }^{27}$ may have limited our ability to assess the extent to which diabetes impacts memory function. 
Previous studies have focused mainly on whether the effects of type 2 diabetes on cognitive performance are independent of cardiovascular risk factors, including hypertension $\left.(e)^{28-31}\right)$. Likewise, various studies have explored whether markers of hyperglycaemia, insulin resistance, and blood pressure are associated with cognitive performance, irrespective of glucose metabolism status $\left(\mathrm{eg},{ }^{12-14}\right)$. Collectively, these studies suggest that both metabolic and vascular factors may be involved in diabetesassociated cognitive decrements. To date, however, evidence on such potential mediating effects of either metabolic or vascular factors is scarce. To our knowledge, one previous study with a relatively small sample size ( $n=499$ of whom only 41 had type 2 diabetes) revealed that repeatedly measured systolic but not diastolic blood pressure was a significant mediator in the association between diabetes and performance on several cognitive tasks. ${ }^{32}$ Potential confounding effects of age, sex, and educational level were, however, not adjusted for.

We are the first to show that hyperglycaemia largely explains diabetes-associated worse cognitive performance, which is in line with a recent study that demonstrated that SAF partially mediates the association of type 2 diabetes with reduced gray matter volume. ${ }^{33}$ From a pathophysiological point of view, intracellular hyperglycaemia induces mitochondrial overproduction of superoxide, ${ }^{5}$ which, in turn, activates multiple pathways through which hyperglycaemia could exert toxic effects on the brain. Specifically, excessive superoxide generation leads to an increased flux of glucose through the polyol and hexosamine pathways, activates protein kinase $\mathrm{C}$ isoforms, increases intracellular calcium concentrations, and augments the advanced glycation of important functional and structural proteins. ${ }^{5,34,35}$ All of these processes may affect brain tissue either directly, by triggering neuronal dysfunction and cell death, ${ }^{6}$ or indirectly, by inducing micro- and/ or macrovascular changes..$^{34,35}$

At first glance, the mediating role of hyperglycaemia observed in the present study seems to contrast with findings of our recent systematic review, ${ }^{36}$ where we showed that measures of hyperglycaemia are only weakly associated with cognitive performance among individuals with type 2 diabetes. This pattern is, however, remarkably similar to that observed for some classic diabetic complications, in that previous studies have shown that glycaemic control is more closely related to the development than to the progression of nephropathy ${ }^{37}$ and retinopathy. ${ }^{38} \mathrm{~A}$ possible explanation for is that hyperglycaemia sets the stage for other (organ-specific) mechanisms to exert their detrimental effects, an explanation that is to some extent supported by our observation of interaction between hyperglycaemia and the use of antihypertensive medication in their association with cognitive performance. 
The present study provides evidence that abnormalities in blood pressure, although to a lesser extent than hyperglycaemia, also contribute to diabetes-associated differences in processing speed. Sensitivity analyses, however, showed that these mediating effects were mainly attributable to the use of antihypertensive medication, and thus to previous exposure to elevated blood pressure rather than the actual blood pressure. Conversely, the frequent use of antihypertensive medication among individuals with type 2 diabetes, which is likely to have contributed to the small differences in 24-hour blood pressure observed between individuals with different glucose metabolism (ie, $<5 \mathrm{mmHg}$ ), may have limited our ability to adequately assess the mediating effects of actual blood pressure in diabetes-associated differences in cognitive performance.

Although our data suggest that insulin resistance does not contribute to diabetesrelated worse cognitive performance, this finding should be interpreted with some caution, primarily because our index of insulin resistance is likely to be less accurate than that of hyperglycaemia and blood pressure-related variables. Apart from the fact that we used a surrogate marker of insulin resistance (ie, HOMA-IR), this marker typically reflects hepatic and muscle insulin resistance, while it has been suggested that these do not necessarily coincide with cerebral insulin resistance. ${ }^{39}$ Consequently, we cannot fully exclude the possibility of cerebral insulin resistance being involved in diabetesassociated cognitive decrements.

The present study has some further limitations that should be noted. First, its crosssectional design does not permit us to draw firm conclusions about the aetiology of diabetes-associated worse cognitive performance. Nonetheless, we believe it is reasonable to infer from our data that hyperglycaemia is likely to play an important role. A second limitation concerns the possibility of residual confounding. Indeed, there might be other factors that contribute to diabetes-associated cognitive decrements, such as low-grade inflammation and abnormalities in the hypothalamic-pituitary-thyroid axis. ${ }^{2}$ Although we cannot fully exclude this possibility, we consider it unlikely as this would imply that such factors need to be associated with cognitive performance and hyperglycaemia, but, at the same time, may not be associated with insulin resistance. Third, the generalisability of our findings can be questioned as individuals with type 2 diabetes in our cohort were generally well controlled for their diabetes and comorbid vascular risk factors. Individuals were, however, not selected on the basis of their glycaemic control, and hence we believe our population is representative for a population that has access to quality diabetes care. Last, autocorrelation between measures of hyperglycaemia and glucose metabolism status may have decreased the statistical power of our study, ${ }^{40}$ contributing to the wide confidence intervals of the mediating effects. However, collinearity diagnostics were negative, and results for hyperglycaemia 


\section{Chapter 2}

were qualitatively similar when the composite index was composed of measures that were not directly used for diabetes classification (Supplement).

In conclusion, our findings suggest that differences in cognitive performance between individuals with type 2 diabetes and those with NGM are largely attributable to hyperglycaemia, and, to a lesser extent, to blood pressure-related variables, whereas insulin resistance has no mediating effects. This is particularly true for the cognitive domains of processing speed and executive function and attention. Differences in memory function were relatively minor and not explained by any of the mediating factors evaluated. Despite the cross-sectional nature of the study, we believe our findings indicate that prevention of diabetes-associated decrements in cognitive performance should focus on early glycaemic and blood pressure control. 


\section{References}

1. Miles, W.E. and H.F. Root, Psychologic tests applied to diabetic patients. Arch Intern Med, 1922. 30(6): p. 767-777.

2. Biessels, G.J., et al., Dementia and cognitive decline in type 2 diabetes and prediabetic stages: towards targeted interventions. Lancet Diabetes Endocrinol, 2014. 2(3): p. 246-55.

3. loacara, S., et al., Improvements in life expectancy in adult type 2 diabetes patients in the last six decades. Diabetes Res Clin Pract, 2011. 92(3): p. 400-4.

4. Strachan, M.W. and J.F. Price, Diabetes. Cognitive decline and T2DM--a disconnect in the evidence? Nat Rev Endocrinol, 2014. 10(5): p. 258-60.

5. Brownlee, M., Biochemistry and molecular cell biology of diabetic complications. Nature, 2001. 414(6865): p. 813-20.

6. Sima, A.A., H. Kamiya, and Z.G. Li, Insulin, C-peptide, hyperglycemia, and central nervous system complications in diabetes. Eur J Pharmacol, 2004. 490(1-3): p. 187-97.

7. Cholerton, B., L.D. Baker, and S. Craft, Insulin, cognition, and dementia. Eur J Pharmacol, 2013. 719(1-3): p. 170-9.

8. Van den Berg, E., et al., Type 2 diabetes mellitus, hypertension, dyslipidemia and obesity: A systematic comparison of their impact on cognition. Biochim Biophys Acta, 2009. 1792(5): p. 470-81.

9. Kloppenborg, R.P., et al., Diabetes and other vascular risk factors for dementia: which factor matters most? A systematic review. Eur J Pharmacol, 2008. 585(1): p. 97-108.

10. Kennelly, S.P., B.A. Lawlor, and R.A. Kenny, Blood pressure and dementia - a comprehensive review. Ther Adv Neurol Disord, 2009. 2(4): p. 241-60.

11. Colosia, A.D., R. Palencia, and S. Khan, Prevalence of hypertension and obesity in patients with type 2 diabetes mellitus in observational studies: a systematic literature review. Diabetes Metab Syndr Obes, 2013. 6: p. 327-38.

12. Crane, P.K., et al., Glucose levels and risk of dementia. N Engl J Med, 2013. 369(6): p. 540-8.

13. Tan, Z.S., et al., Association of metabolic dysregulation with volumetric brain magnetic resonance imaging and cognitive markers of subclinical brain aging in middle-aged adults: the Framingham Offspring Study. Diabetes Care, 2011. 34(8): p. 1766-70.

14. Kilander, L., et al., Hypertension is related to cognitive impairment: a 20-year follow-up of 999 men. Hypertension, 1998. 31(3): p. 780-6.

15. Schram, M.T., et al., The Maastricht Study: an extensive phenotyping study on determinants of type 2 diabetes, its complications and its comorbidities. Eur J Epidemiol, 2014. 29(6): p. 439-51.

16. Geijselaers, S.L., Carotid stiffness is associatied with impairment of cognitive performance in individuals with and without type 2 diabetes - The Maastricht Study. Atherosclerosis, 2016. In press.

17. Wallace, T.M., J.C. Levy, and D.R. Matthews, Use and abuse of HOMA modeling. Diabetes Care, 2004. 27(6): p. 1487-95.

18. Horwitz, D.L., et al., Proinsulin, insulin, and C-peptide concentrations in human portal and peripheral blood. J Clin Invest, 1975. 55(6): p. 1278-83.

19. Ohkura, T., et al., 20/(fasting C-peptide $x$ fasting plasma glucose) is a simple and effective index of insulin resistance in patients with type 2 diabetes mellitus: a preliminary report. Cardiovasc Diabetol, 2013. 12: p. 21.

20. Sabayan, B., et al., Association of visit-to-visit variability in blood pressure with cognitive function in old age: prospective cohort study. BMJ, 2013. 347: p. f4600.

21. Alperovitch, A., et al., Blood pressure variability and risk of dementia in an elderly cohort, the Three-City Study. Alzheimers Dement, 2014. 10(5 Suppl): p. S330-7.

22. Van Sloten, T.T., et al., Association between arterial stiffness, cerebral small vessel disease and cognitive impairment: A systematic review and meta-analysis. Neurosci Biobehav Rev, 2015. 


\section{Chapter 2}

53: p. 121-30.

23. Preacher, K.J. and A.F. Hayes, Asymptotic and resampling strategies for assessing and comparing indirect effects in multiple mediator models. Behav Res Methods, 2008. 40(3): p. 879-91.

24. Rothman, K.J., No adjustments are needed for multiple comparisons. Epidemiology, 1990. 1(1): p. 43-6.

25. Palta, P., et al., Magnitude of cognitive dysfunction in adults with type 2 diabetes: a metaanalysis of six cognitive domains and the most frequently reported neuropsychological tests within domains. J Int Neuropsychol Soc, 2014. 20(3): p. 278-91.

26. Koekkoek, P.S., et al., Cognitive function in patients with diabetes mellitus: guidance for daily care. Lancet Neurol, 2015. 14(3): p. 329-40.

27. Stern, Y., Cognitive reserve. Neuropsychologia, 2009. 47(10): p. 2015-28.

28. Peila, R., et al., Type 2 diabetes, APOE gene, and the risk for dementia and related pathologies: The Honolulu-Asia Aging Study. Diabetes, 2002. 51(4): p. 1256-62.

29. MacKnight, C., et al., Diabetes mellitus and the risk of dementia, Alzheimer's disease and vascular cognitive impairment in the Canadian Study of Health and Aging. Dement Geriatr Cogn Disord, 2002. 14(2): p. 77-83.

30. Fontbonne, A., et al., Changes in cognitive abilities over a 4-year period are unfavorably affected in elderly diabetic subjects: results of the Epidemiology of Vascular Aging Study. Diabetes Care, 2001. 24(2): p. 366-70.

31. Kanaya, A.M., et al., Change in cognitive function by glucose tolerance status in older adults: a 4-year prospective study of the Rancho Bernardo study cohort. Arch Intern Med, 2004. 164(12): p. 1327-33.

32. McFall, G.P., et al., Testing covariates of Type 2 diabetes-cognition associations in older adults: moderating or mediating effects? Neuropsychology, 2010. 24(5): p. 547-62.

33. Moran, C., et al., Type 2 diabetes, skin autofluorescence, and brain atrophy. Diabetes, 2015. 64(1): p. 279-83.

34. Biessels, G.J., et al., Risk of dementia in diabetes mellitus: a systematic review. Lancet Neurol, 2006. 5(1): p. 64-74.

35. Gispen, W.H. and G.J. Biessels, Cognition and synaptic plasticity in diabetes mellitus. Trends Neurosci, 2000. 23(11): p. 542-9.

36. Geijselaers, S.L., et al., Glucose regulation, cognition, and brain MRI in type 2 diabetes: a systematic review. Lancet Diabetes Endocrinol, 2015. 3(1): p. 75-89.

37. Fioretto, P., et al., Renal protection in diabetes: role of glycemic control. J Am Soc Nephrol, 2006. 17(4 Suppl 2): p. S86-9.

38. Calcutt, N.A., et al., Therapies for hyperglycaemia-induced diabetic complications: from animal models to clinical trials. Nat Rev Drug Discov, 2009. 8(5): p. 417-29.

39. Banks, W.A., J.B. Owen, and M.A. Erickson, Insulin in the brain: there and back again. Pharmacol Ther, 2012. 136(1): p. 82-93.

40. Beasley, T.M., Tests of Mediation: Paradoxical Decline in Statistical Power as a Function of Mediator Collinearity. J Exp Educ, 2014. 82(3): p. 283-306. 
Exploring the aetiology of diabetes-associated worse cognitive performance 





Supplement to

Chapter 2 


\section{Extended methods}

\section{Study population}

The Maastricht Study ${ }^{1}$ focuses on the aetiology, pathophysiology, complications, and comorbidities of type 2 diabetes and is characterised by an extensive phenotyping approach. Individuals eligible to participate are those aged between 40 and 75 years who live in the southern part of the Netherlands and are sufficiently proficient in the Dutch language. Participants are recruited through mass media campaigns and from the municipal registries and the regional Diabetes Patient Registry via mailings. Recruitment is stratified according to known diabetes status for reasons of efficiency.

\section{Glucose metabolism status}

A standardised two-hour seven-sample oral glucose tolerance test (OGTT) was used to determine the participants' glucose metabolism status. ${ }^{1}$ According to the 2006 WHO diagnostic criteria, ${ }^{2}$ glucose metabolism status was classified as normal glucose metabolism (NGM), prediabetes (impaired glucose metabolism), or diabetes. Participants who were prescribed glucose-lowering medication without a prior diagnosis of type 1 diabetes were considered to have type 2 diabetes, regardless of OGTT results. For safety reasons, participants on insulin and those with a fasting glucose level above $11.0 \mathrm{mmol} / \mathrm{L}$ were excluded from the OGTT. ${ }^{1}$

\section{Cognitive performance}

The composite memory score was derived from the Verbal Learning Test $^{3}$ by averaging total immediate and delayed recall scores. The composite score for information processing speed was derived from the Stroop Colour Word Test ${ }^{4}$ Part I and II, the Concept Shifting Test ${ }^{5}$ Part A and B, and the Letter-Digit Substitution Test. ${ }^{6}$ Executive function and attention was assessed by the Stroop Colour Word Test Part III and the Concept Shifting Test Part C. Where necessary, individual test scores were inverted so that higher scores indicated better cognitive performance.

\section{Hyperglycaemia and blood pressure-related variables}

\section{Hyperglycaemia}

Venous fasting and post-load plasma glucose levels were measured by the enzymatic hexokinase method on two automatic analysers (ie, the Beckman Synchron LX20 [Beckman Coulter Inc., USA] for samples obtained between November 2010 and April 2012, and the Roche Cobas 6000 [Roche Diagnostics, Mannheim, Germany] for samples obtained thereafter). HbA1c was determined by ion-exchange high performance liquid chromatography. SAF, a marker of tissue advanced glycation end-product accumulation, 
was measured with the AGE reader (DiagnOptics Technologies B.V., Groningen, the Netherlands). Details on the AGE reader measurements, including data on their reproducibility, have been published previously. ${ }^{7}$

\section{Blood pressure-related variables}

Participants were requested to undergo 24-hour blood pressure monitoring (WatchBP03, Microlife AG, Widnau, Switzerland) with an appropriate sized cuff placed around their non-dominant arm. ${ }^{1,8}$ The device was programmed to take blood pressure readings every 15 minutes during the day (08.00AM-11.00PM) and every 30 minutes at night (11.00PM-08.00AM). According to recommendations of the British Hypertension Society, ${ }^{9}$ average 24 -hour blood systolic and diastolic blood pressure were only calculated in case there were more than 14 valid blood pressure reading during daytime and more than seven valid readings during night-time. Short-term systolic and diastolic blood pressure variability were determined by the weighted standard deviation of the 24-hour systolic and diastolic blood pressure measurements, respectively, as described in detail elsewhere. ${ }^{8,10}$ The use of antihypertensive medication was ascertained by a medication interview. To this end, participants were ask to bring their prescribed and over-thecounter medication to the research centre and a trained staff member registered the generic name and dose of the medication.

\section{Covariates}

Educational level, classified as low/intermediate/high, was ascertained during a personal interview conducted shortly before the cognitive assessment. ${ }^{1}$ Participants were instructed to bring their medication to the research centre, and research nurses recorded all current medications. ${ }^{1}$ Details on the assessment of cardiovascular risk factors (ie, waist circumference, total/high-density lipoprotein cholesterol ratio, smoking behaviour, and history of cardiovascular disease) can be found in previous reports. ${ }^{1,8,11}$ The presence of depressive symptoms was evaluated with use of the Mini International Neuropsychiatric Interview (MINI). ${ }^{12}$ For the present study, a current depression was defined as the presence of at least one core symptom (ie, depressed mood and loss of interest) along with one to three sub-symptoms (ie, change in appetite, sleep disturbances, irregular motor movements or speech, loss of usual energy, feeling of being worthless or guilty, difficulty concentrating or decision making, and self-deprecatory ideation).

\section{Sensitivity analyses}

Multiple sensitivity analyses were performed. Firstly, we evaluated whether the extent of mediation differed between individuals with newly diagnosed type 2 diabetes and those with a known diagnosis of diabetes at study entry. Secondly, we re-analysed the data excluding participants treated with exogenous insulin as the HOMA-IR index has 
not been validated adequately for individuals on insulin, ${ }^{13}$ although recent research suggests that it might be valid. ${ }^{14}$ Thirdly, the mediating effects of hyperglycaemia, insulin resistance, and blood pressure-related variables were explored with additional adjustments for traditional cardiovascular risk factors (ie, waist circumference, total/highdensity lipoprotein cholesterol ratio, smoking behaviour, and history of cardiovascular disease) and the presence of a depression. We also evaluated the mediating effects of these additional variables on diabetes-associated differences in cognitive performance.

Fourthly, we investigated whether the results depended on how the composite indices were constructed. To this end, as outlined in the main text (1) the mediating effects of hyperglycaemia were re-explored with focus on long-term hyperglycaemia; (2) the mediating effects of insulin resistance were re-analysed with $\mathrm{C}$-peptide rather than fasting insulin to calculate HOMA-IR; (3) the mediating effects of insulin resistance were re-analysed with an index that combined the HOMA-IR indices as calculated with fasting insulin and C-peptide levels; and (4) the mediating effects of blood pressurerelated variables were re-analysed with an composite index that included 24-hour pulse pressure rather than 24-hour systolic and diastolic blood pressure.

Fifthly, because previous studies have shown that fasting glucose ${ }^{15,16}$ and blood pressure ${ }^{8,}$ ${ }^{17}$ may be quadratically associated with cognitive performance, we tested for presence of such quadratic associations between, on the one hand, the individual components of hyperglycaemia, insulin resistance, and blood pressure-related variables, and, on the other, cognitive performance by entering linear and quadratic terms of the meancentred variables to the regression model. In case of a statistically significant quadratic association, the mediating effects of that variable were reconsidered with use of a SPSS macro that could account for non-linearity. ${ }^{18}$ Finally, we assessed the effects of our complete case analysis approach by using all available data, ie, composite indices were used in the analyses when participants had data on at least one of the individuals components of these indices.

\section{Extended results}

\section{Sensitivity analyses}

Results were qualitatively similar when individuals with newly diagnosed type 2 diabetes were analysed separately from those with a prior diagnoses of diabetes, or when individuals treated with insulin were excluded from the analyses (data not shown). Overall, additional adjustment for prior cardiovascular disease, cardiovascular risk factors and depression reduced diabetes-related worse cognitive performance (by $62.9 \%$ for memory function, $13.4 \%$ for processing speed, and $1.1 \%$ for executive 
function and attention). However, the pattern of mediation by hyperglycaemia, insulin resistance, and blood pressure-related variables was comparable to that observed after adjustment for age, sex, and educational level only, except that the mediating effects of hyperglycaemia were somewhat smaller and not statistically significant for executive function and attention (Figure S2.2). Additional examination of the mediating effects of the cardiovascular risk factors revealed that only waist circumference and a history of cardiovascular disease statistically significantly mediated diabetes-related worse performance in memory function and executive function and attention, respectively (Figure S2.3). The presence of a depression mediated, albeit marginally, diabetes-related worse performance in all the cognitive domains assessed (Figure S2.3).

When the composite index of hyperglycaemia was constructed by averaging $\mathrm{HbA1c}$ and SAF, and thus focused solely on long-term hyperglycaemia, its mediating effects became somewhat smaller, as became their $95 \%$ confidence intervals, indicating more accurate estimations (Figure S2.4). The latter likely resulted from the fact that longterm hyperglycaemia correlated less strongly with the presence of diabetes (Pearson correlation coefficient 0.60 ) than the composite construct that also included shortterm hyperglycaemia (0.79). Results were very similar to those of the main analysis when HOMA-IR was calculated with fasting C-peptide instead of insulin levels (data not shown); insulin resistance was reflected by a composite index of the HOMA-IR indices as calculated with fasting insulin and C-peptide levels (Figure S2.5); or 24-hour pulse pressure rather than 24-hour systolic and diastolic blood pressure was incorporated in the index of blood pressure abnormalities (Figure S2.6).

When we tested for the presence of quadratic associations, we only found such nonlinear associations for fasting plasma glucose, HOMA-IR, and 24-hour systolic blood pressure with processing speed, with somewhat larger mediating effects (Figure S2.7). Finally, we explored the effects of our complete case analysis approach and found that a similar pattern of mediating would have been found had we used all the available data (Figure S2.8). 
Chapter 2 


\section{References}

1. Schram, M.T., et al., The Maastricht Study: an extensive phenotyping study on determinants of type 2 diabetes, its complications and its comorbidities. Eur J Epidemiol, 2014. 29(6): p. 439-51.

2. World Health Organisation, W.H. Definition and diagnosis of diabetes mellitus and intermediate hyperglycemia: report of a WHO/IDF consultation. 2006. Available at: http:// www.idf.org/webdata/docs/WHO_IDF_definition_diagnosis_of_diabetes.pdf. Last accessed August 4, 2015.

3. Van der Elst, W., et al., Rey's verbal learning test: normative data for 1855 healthy participants aged 24-81 years and the influence of age, sex, education, and mode of presentation. J Int Neuropsychol Soc, 2005. 11(3): p. 290-302.

4. Van der Elst, W., et al., The Stroop color-word test: influence of age, sex, and education; and normative data for a large sample across the adult age range. Assessment, 2006. 13(1): p. 6279.

5. Van der Elst, W., et al., The Concept Shifting Test: adult normative data. Psychol Assess, 2006. 18(4): p. 424-32.

6. Van der Elst, W., et al., The Letter Digit Substitution Test: normative data for 1,858 healthy participants aged 24-81 from the Maastricht Aging Study (MAAS): influence of age, education, and sex. J Clin Exp Neuropsychol, 2006. 28(6): p. 998-1009.

7. Spauwen, P.J., et al., Associations of advanced glycation end-products with cognitive functions in individuals with and without type 2 diabetes: the maastricht study. J Clin Endocrinol Metab, 2015. 100(3): p. 951-60.

8. Spauwen, P.J., et al., Both Low and High 24-Hour Diastolic Blood Pressure Are Associated With Worse Cognitive Performance in Type 2 Diabetes: The Maastricht Study. Diabetes Care, 2015. 38(8): p. 1473-80.

9. O'Brien, E., et al., Use and interpretation of ambulatory blood pressure monitoring: recommendations of the British hypertension society. BMJ, 2000. 320(7242): p. 1128-34.

10. Bilo, G., et al., A new method for assessing 24-h blood pressure variability after excluding the contribution of nocturnal blood pressure fall. J Hypertens, 2007. 25(10): p. 2058-66.

11. Geijselaers, S.L., Carotid stiffness is associatied with impairment of cognitive performance in individuals with and without type 2 diabetes - The Maastricht Study. Atherosclerosis, 2016. In press.

12. Sheehan, D.V., et al., The Mini-International Neuropsychiatric Interview (M.I.N.I.): the development and validation of a structured diagnostic psychiatric interview for DSM-IV and ICD-10. J Clin Psychiatry, 1998. 59 Suppl 20: p. 22-33;quiz 34-57.

13. Wallace, T.M., J.C. Levy, and D.R. Matthews, Use and abuse of HOMA modeling. Diabetes Care, 2004. 27(6): p. 1487-95.

14. Okita, K., et al., Homeostasis model assessment of insulin resistance for evaluating insulin sensitivity in patients with type 2 diabetes on insulin therapy. Endocr J, 2013. 60(3): p. 28390.

15. Shorr, R.I., et al., Glycemia and cognitive function in older adults using glucose-lowering drugs. J Nutr Health Aging, 2006. 10(4): p. 297-301.

16. Gallacher, J.E., et al., Glucoregulation has greater impact on cognitive performance than macro-vascular disease in men with type 2 diabetes: data from the Caerphilly study. Eur J Epidemiol, 2005. 20(9): p. 761-8.

17. Waldstein, S.R., et al., Nonlinear relations of blood pressure to cognitive function: the Baltimore Longitudinal Study of Aging. Hypertension, 2005. 45(3): p. 374-9.

18. Hayes, A.F. and K.J. Preacher, Quantifying and Testing Indirect Effects in Simple Mediation Models When the Constituent Paths are Nonlinear. Multivariate Behavioral Research, 2010. 45(4): p. 32 
Table S2.1 Characteristics of individuals included and those excluded from the main analyses

\begin{tabular}{|c|c|c|c|c|}
\hline Characteristics & $\begin{array}{l}\text { Included } \\
(n=2531)\end{array}$ & $\begin{array}{c}\text { Excluded } \\
\left(n_{\max }=879\right)\end{array}$ & Missing ${ }^{*}$ & p-value ${ }^{+}$ \\
\hline Age (years) & $60 \pm 8$ & $60 \pm 8$ & 0 & 0.853 \\
\hline Male & 1314 (52.0\%) & 442 (50.3\%) & 0 & 0.404 \\
\hline Educational level & $388 / 1110 / 1033$ & $172 / 304 / 293$ & 110 & $<0.001$ \\
\hline (low/middle/high) & $(15.3 \% / 43.9 \% / 40.8 \%)$ & $(22.4 \% / 39.5 \% / 38.1 \%)$ & & \\
\hline Fasting glucose (mmol/L) & $5.5[5.1-6.4]$ & $5.6[5.1-6.9]$ & 1 & $<0.001$ \\
\hline 2-hour post OGTT glucose (mmol/L) & $6.2[5.1-9.1]$ & $6.5[5.1-10.0]$ & 108 & 0.069 \\
\hline $\mathrm{HbA} 1 \mathrm{c}(\%)$ & $5.9 \pm 0.8$ & $6.1 \pm 1.1$ & 13 & $<0.001$ \\
\hline $\mathrm{HbA} 1 \mathrm{c}(\mathrm{mmol} / \mathrm{mol})$ & $41 \pm 9$ & $43 \pm 12$ & 13 & $<0.001$ \\
\hline SAF (AU) & $2.44 \pm 0.54$ & $2.43 \pm 0.57$ & 198 & 0.824 \\
\hline Glucose metabolism status & $1479 / 386 / 666$ & $445 / 125 / 309$ & 0 & $<0.001$ \\
\hline (NGM/prediabetes/T2DM) & (58.4\%/15.3\%/26.3\%) & $(50.6 \% / 14.2 \% / 35.2 \%)$ & & \\
\hline Fasting insulin (pmol/L) & $61[42-92]$ & $61[43-99]$ & 45 & 0.219 \\
\hline Fasting C-peptide (nmol/L) & $0.67 \pm 0.32$ & $0.72 \pm 0.39$ & 46 & 0.003 \\
\hline HOMA-IR (AU) & $1.2[0.8-1.8]$ & $1.2[0.8-2.0]$ & 49 & 0.011 \\
\hline 24-hour SBP (mmHg) & $119 \pm 12$ & $120 \pm 13$ & 396 & 0.114 \\
\hline 24-hour DBP (mmHg) & $73 \pm 7$ & $74 \pm 7$ & 396 & 0.262 \\
\hline Weighted 24-hour SD of SBP (mmHg) & $11[9-13]$ & 11 [9-13] & 468 & 0.367 \\
\hline Weighted 24-hour SD of DBP ( $\mathrm{mmHg}$ ) & $8[7-10]$ & 9 [7-10] & 468 & 0.035 \\
\hline Hypertension & $1412(55.8 \%)$ & $503(57.6 \%)$ & 6 & 0.347 \\
\hline Antihypertensive medication & $978(38.6 \%)$ & 377 (43.1\%) & 4 & 0.021 \\
\hline Waist circumference $(\mathrm{cm})$ & $95 \pm 13$ & $97 \pm 15$ & 1 & 0.001 \\
\hline Total cholesterol (mmol/L) & $5.3 \pm 1.2$ & $5.1 \pm 1.2$ & 4 & 0.006 \\
\hline HDL-cholesterol (mmol/L) & $1.5 \pm 0.5$ & $1.5 \pm 0.5$ & 4 & 0.657 \\
\hline LDL-cholesterol (mmol/L) & $3.1 \pm 1.0$ & $3.0 \pm 1.0$ & 4 & $<0.001$ \\
\hline Triglycerides (mmol/L) & $1.2[0.9-1.7]$ & $1.2[0.9-1.8]$ & 4 & 0.017 \\
\hline Lipid-modifying medication & $879(34.7 \%)$ & $341(39.0 \%)$ & 4 & 0.024 \\
\hline Smoking behaviour & $870 / 1304 / 313$ & $290 / 425 / 147$ & 17 & 0.004 \\
\hline (never/former/current) & (35.0\%/52.4\%/12.6\%) & (33.6\%/49.3\%/17.1\%) & & \\
\hline Prior cardiovascular disease(s) & $399(16.3 \%)$ & $153(18.0 \%)$ & 28 & 0.246 \\
\hline Current depression & $136(5.4 \%)$ & $44(5.8 \%)$ & 125 & 0.655 \\
\hline Memory & $0.02 \pm 0.95$ & $-0.07 \pm 0.98$ & 145 & 0.023 \\
\hline Processing speed & $0.03 \pm 0.77$ & $-0.02 \pm 0.83$ & 184 & 0.157 \\
\hline Executive function \& attention & $0.02 \pm 0.81$ & $-0.03 \pm 0.85$ & 204 & 0.108 \\
\hline
\end{tabular}

Data are presented as mean \pm standard deviation, median [interquartile range], or $\mathrm{n}(\%)$. *Number of missings for a specific variable. ${ }^{\dagger}$ P-value for difference between individuals included and those excluded from the main analyses as determined by independent-samples t-tests for continuous variables and chi-square tests for categorical variables. Abbreviations: $\mathrm{NGM}=$ normal glucose metabolism; T2DM = type 2 diabetes; OGTT = oral glucose tolerance test; HbA1c = glycated haemoglobin; $\mathrm{SAF}=$ skin autofluorescence; $\mathrm{AU}=$ arbitrary units; $\mathrm{HOMA}-\mathrm{IR}=$ homeostasis model assessment of insulin resistance; $\mathrm{SBP}=$ systolic blood pressure; $\mathrm{DBP}=$ diastolic blood pressure; $\mathrm{SD}=$ standard deviation; $\mathrm{HDL}=\mathrm{high}-$ density lipoprotein. 
Table S2.2 Associations between indices used for hyperglycaemia, insulin resistance, and blood pressure-related variables and cognitive performance

\begin{tabular}{|c|c|c|c|}
\hline & Memory & Processing speed & $\begin{array}{l}\text { Executive function } \\
\quad \& \text { attention }\end{array}$ \\
\hline Hyperglycaemia & $\begin{array}{c}-0.029 \\
(-0.065 \text { to } 0.006)\end{array}$ & $\begin{array}{c}-0.102^{*} \\
(-0.129 \text { to }-0.074)\end{array}$ & $\begin{array}{c}-0.083^{*} \\
(-0.114 \text { to }-0.053)\end{array}$ \\
\hline Insulin resistance & $\begin{array}{c}-0.029 \\
(-0.062 \text { to } 0.003)\end{array}$ & $\begin{array}{c}-0.024 \\
(-0.050 \text { to } 0.001)\end{array}$ & $\begin{array}{c}-0.041^{*} \\
(-0.070 \text { to }-0.013)\end{array}$ \\
\hline \multicolumn{4}{|c|}{ Blood pressure-related variables } \\
\hline 24-hour BP and their SD & $\begin{array}{c}0.001 \\
(-0.033 ; 0.034)\end{array}$ & $\begin{array}{c}-0.014 \\
(-0.040 ; 0.012)\end{array}$ & $\begin{array}{c}-0.010 \\
(-0.039 ; 0.019)\end{array}$ \\
\hline $\begin{array}{l}\text { Use of antihypertensive } \\
\text { medication }\end{array}$ & $\begin{array}{c}-0.085^{*} \\
(-0.155 ;-0.015)\end{array}$ & $\begin{array}{c}-0.131^{*} \\
(-0186 ;-0.076)\end{array}$ & $\begin{array}{c}-0.081^{*} \\
(-0.142 ;-0.020)\end{array}$ \\
\hline
\end{tabular}

$\mathrm{N}=2531$. Data are presented as unstandardised regression coefficients $(95 \% \mathrm{Cl})$. All analyses are adjusted for age, sex, and educational level. Regression coefficients of 24-hour BP and their SD are additionally adjusted for the use of antihypertensive medication, and vice versa. ${ }^{*} \mathrm{p}<0.05$. Abbreviations: $\mathrm{BP}=$ blood pressure; $\mathrm{SD}=$ standard deviation. 
Table S2.3 Associations between individual components of composite indices and cognitive performance

\begin{tabular}{|c|c|c|c|}
\hline & Memory & Processing speed & $\begin{array}{l}\text { Executive function } \\
\quad \& \text { attention }\end{array}$ \\
\hline $\begin{array}{l}\text { (In) Fasting glucose } \\
(\mathrm{mmol} / \mathrm{l})\end{array}$ & $\begin{array}{c}-0.118 \\
(-0.284 ; 0.048)\end{array}$ & $\begin{array}{c}-0.366^{*} \\
(-0.496 ;-0.237)\end{array}$ & $\begin{array}{c}-0.279^{*} \\
(-0.423 ;-0.134)\end{array}$ \\
\hline $\begin{array}{l}\text { (In) Post-load glucose } \\
(\mathrm{mmol} / \mathrm{l})^{\dagger}\end{array}$ & $\begin{array}{c}-0.073 \\
(-0.148 ; 0.002)\end{array}$ & $\begin{array}{c}-0.113^{*} \\
(-0.171 ;-0.055)\end{array}$ & $\begin{array}{c}-0.113^{*} \\
(-0.178 ;-0.048)\end{array}$ \\
\hline HbA1c (\%) & $\begin{array}{c}-0.009 \\
(-0.049 ; 0.032)\end{array}$ & $\begin{array}{c}-0.102^{*} \\
(-0.133 ;-0.070)\end{array}$ & $\begin{array}{c}-0.080^{*} \\
(-0.115 ;-0.045)\end{array}$ \\
\hline $\begin{array}{l}\text { Skin autofluorescence } \\
(A U)\end{array}$ & $\begin{array}{c}-0.025 \\
(-0.088 ; 0.038)\end{array}$ & $\begin{array}{c}-0.109^{*} \\
(-0.158 ;-0.059)\end{array}$ & $\begin{array}{c}-0.110^{*} \\
(-0.165 ;-0.055)\end{array}$ \\
\hline (In) HOMA-IR (AU) & $\begin{array}{c}-0.083 \\
(-0.175 ; 0.010)\end{array}$ & $\begin{array}{c}-0.069 \\
(-0.142 ; 0.003)\end{array}$ & $\begin{array}{c}-0.116^{*} \\
(-0.197 ;-0.036)\end{array}$ \\
\hline $\begin{array}{l}\text { 24-hour Systolic blood } \\
\text { pressure }(\mathrm{mmHg})\end{array}$ & $\begin{array}{c}-0.000 \\
(-0.003 ; 0.003)\end{array}$ & $\begin{array}{c}0.000 \\
(-0.002 ; 0.003)\end{array}$ & $\begin{array}{c}-0.000 \\
(-0.003 ; 0.002)\end{array}$ \\
\hline $\begin{array}{l}\text { 24-hour Diastolic blood } \\
\text { pressure }(\mathrm{mmHg})\end{array}$ & $\begin{array}{c}-0.001 \\
(-0.006 ; 0.004)\end{array}$ & $\begin{array}{c}0.003 \\
(-0.001 ; 0.007)\end{array}$ & $\begin{array}{c}-0.000 \\
(-0.005 ; 0.004)\end{array}$ \\
\hline $\begin{array}{l}\text { (In) Weighted SD systolic } \\
\text { blood pressure (mmHg) }\end{array}$ & $\begin{array}{c}0.040 \\
(-0.089 ; 0.169)\end{array}$ & $\begin{array}{c}-0.058 \\
(-0.160 ; 0.043)\end{array}$ & $\begin{array}{c}0.020 \\
(-0.093 ; 0.132)\end{array}$ \\
\hline $\begin{array}{l}\text { (In) Weighted SD diastolic } \\
\text { blood pressure }(\mathrm{mmHg})\end{array}$ & $\begin{array}{c}-0.038 \\
(-0.156 ; 0.080)\end{array}$ & $\begin{array}{c}-0.214^{*} \\
(-0.306 ;-0.122)\end{array}$ & $\begin{array}{c}-0.117^{*} \\
(-0.219 ;-0.015)\end{array}$ \\
\hline $\begin{array}{l}\text { Use of antihypertensive } \\
\text { medication (yes vs. no) }\end{array}$ & $\begin{array}{c}-0.085^{*} \\
(-0.155 ;-0.015)\end{array}$ & $\begin{array}{c}-0.133^{*} \\
(-0.188 ;-0.079)\end{array}$ & $\begin{array}{c}-0.083^{*} \\
(-0.143 ;-0.022)\end{array}$ \\
\hline
\end{tabular}

$\mathrm{N}=2531$. Data are presented as unstandardised regression coefficients $(95 \% \mathrm{Cl})$. All analyses are adjusted for age, sex, and educational level. ${ }^{*} p<0.05$. ${ }^{+} n=2388$. Abbreviations: In = natural logarithm; $A U$ = arbitrary units; SD = standard deviation. 


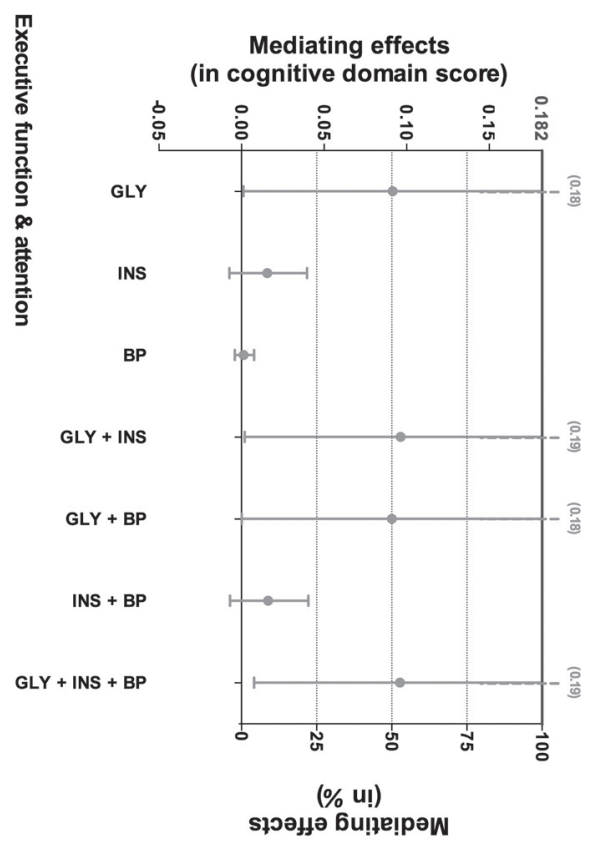

产

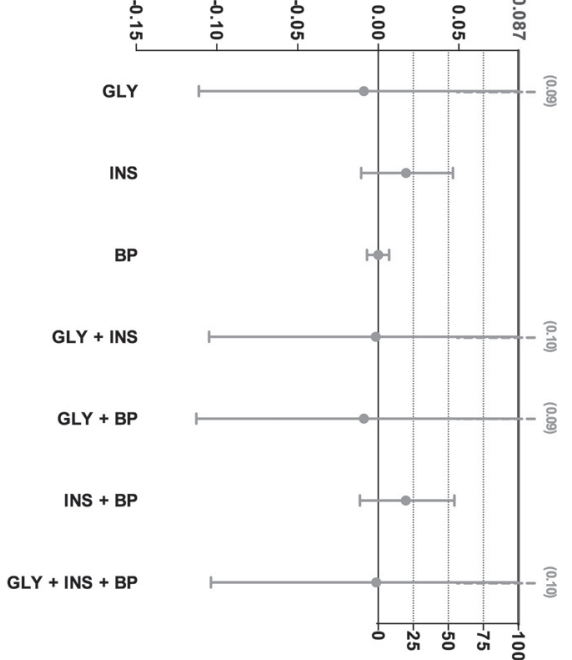

$\stackrel{2}{3} \frac{1}{10}$

을 일

骂

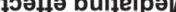

(\% u!)

słəәңә Бu!ฺe!pәw

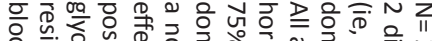

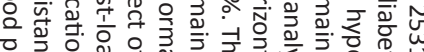

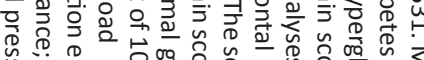

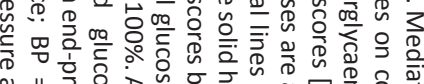

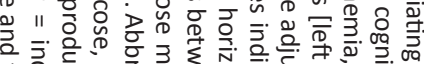

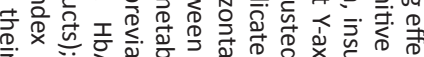

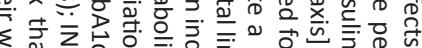
离. 峲

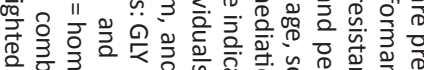
문.

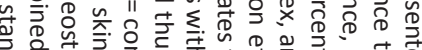

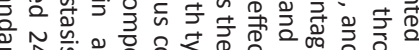

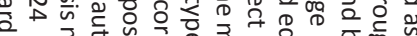

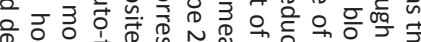

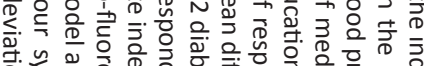

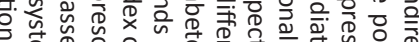

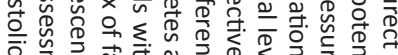

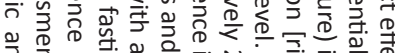

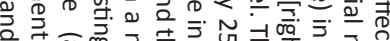

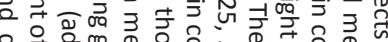

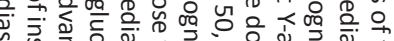

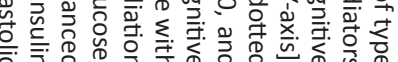

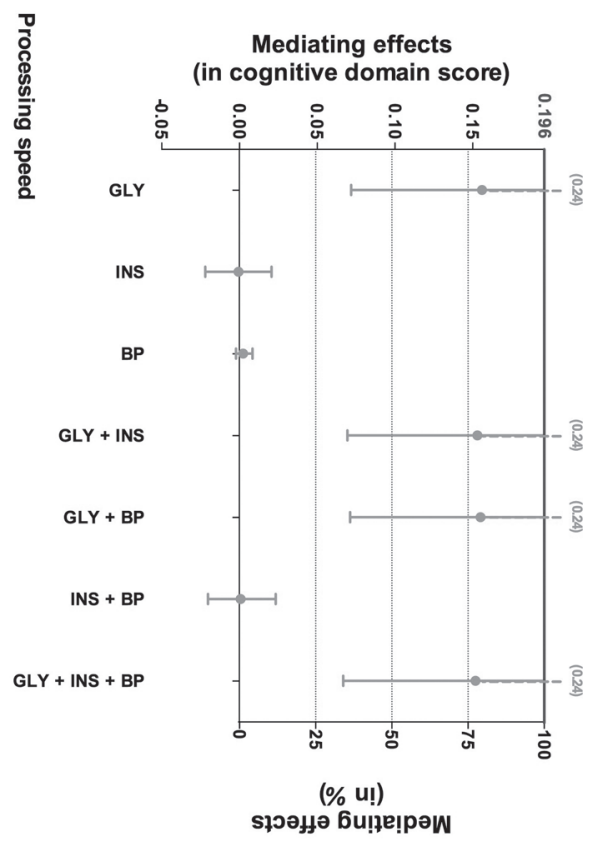

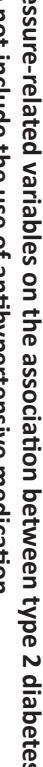



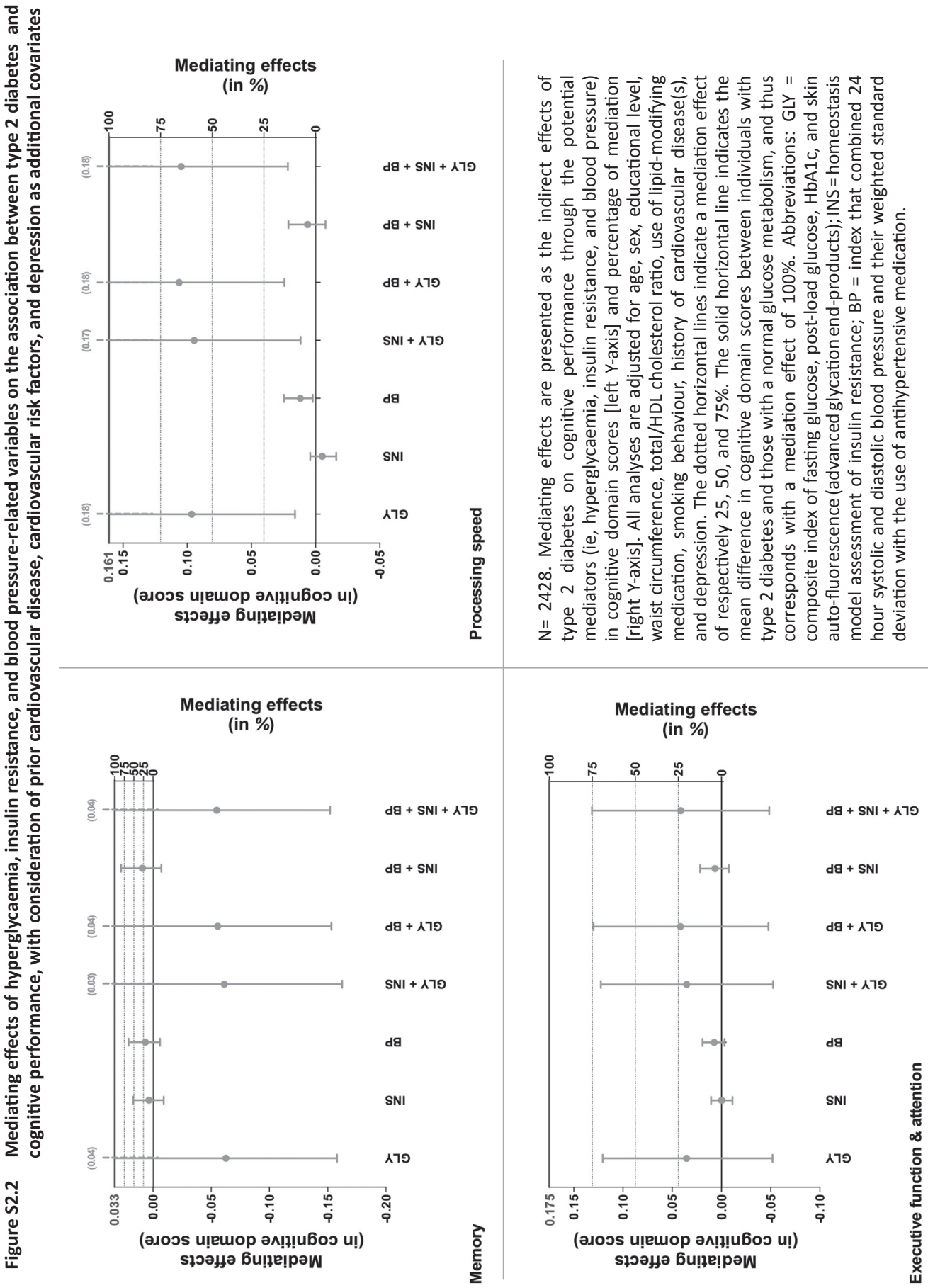

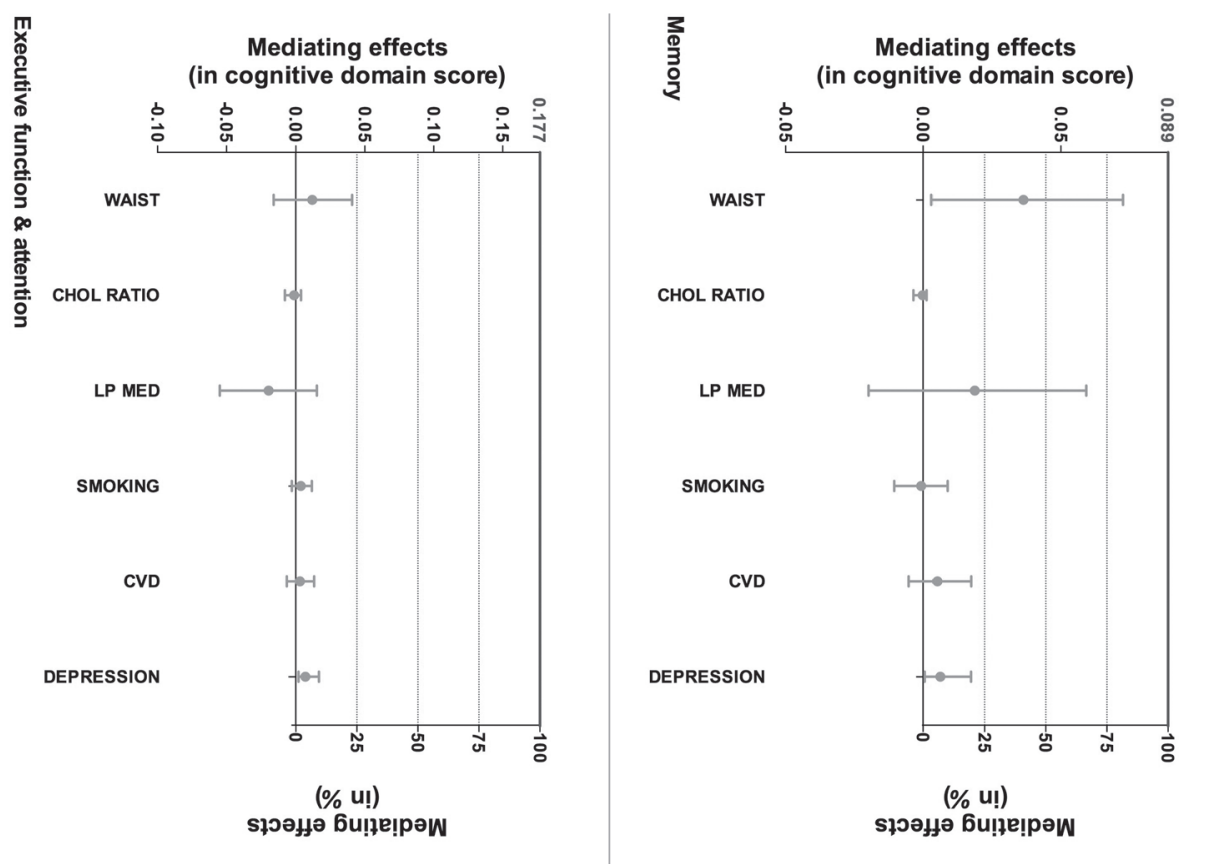

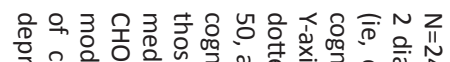

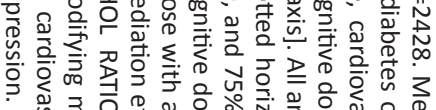

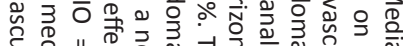

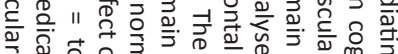

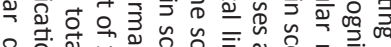

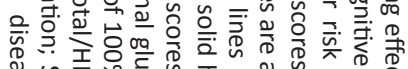

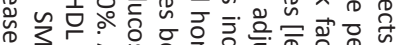

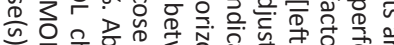

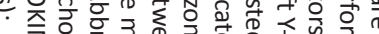

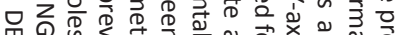

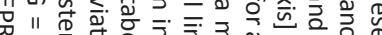

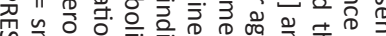

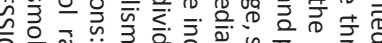

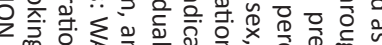

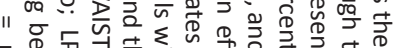

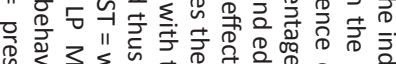

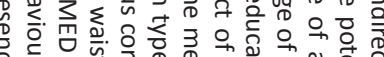

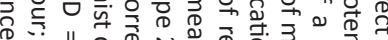

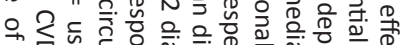
ט

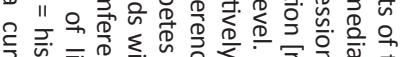

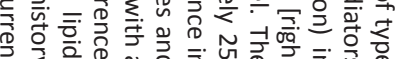

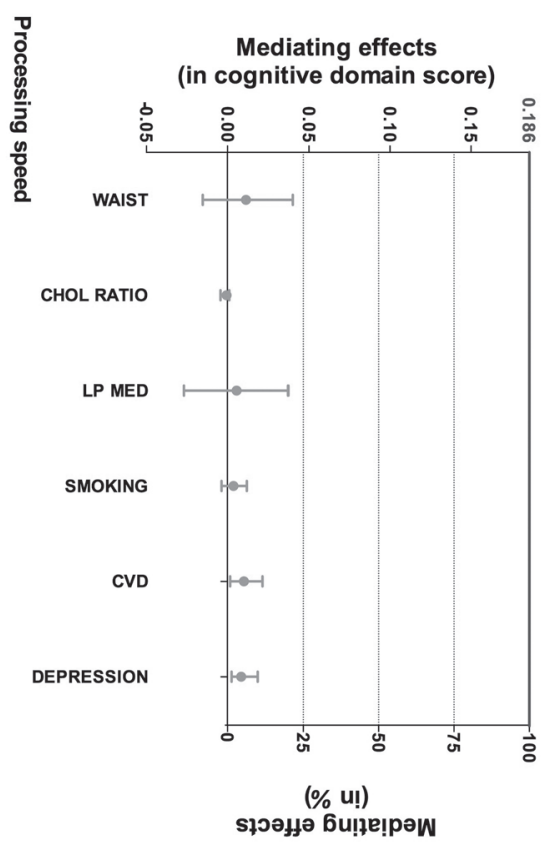



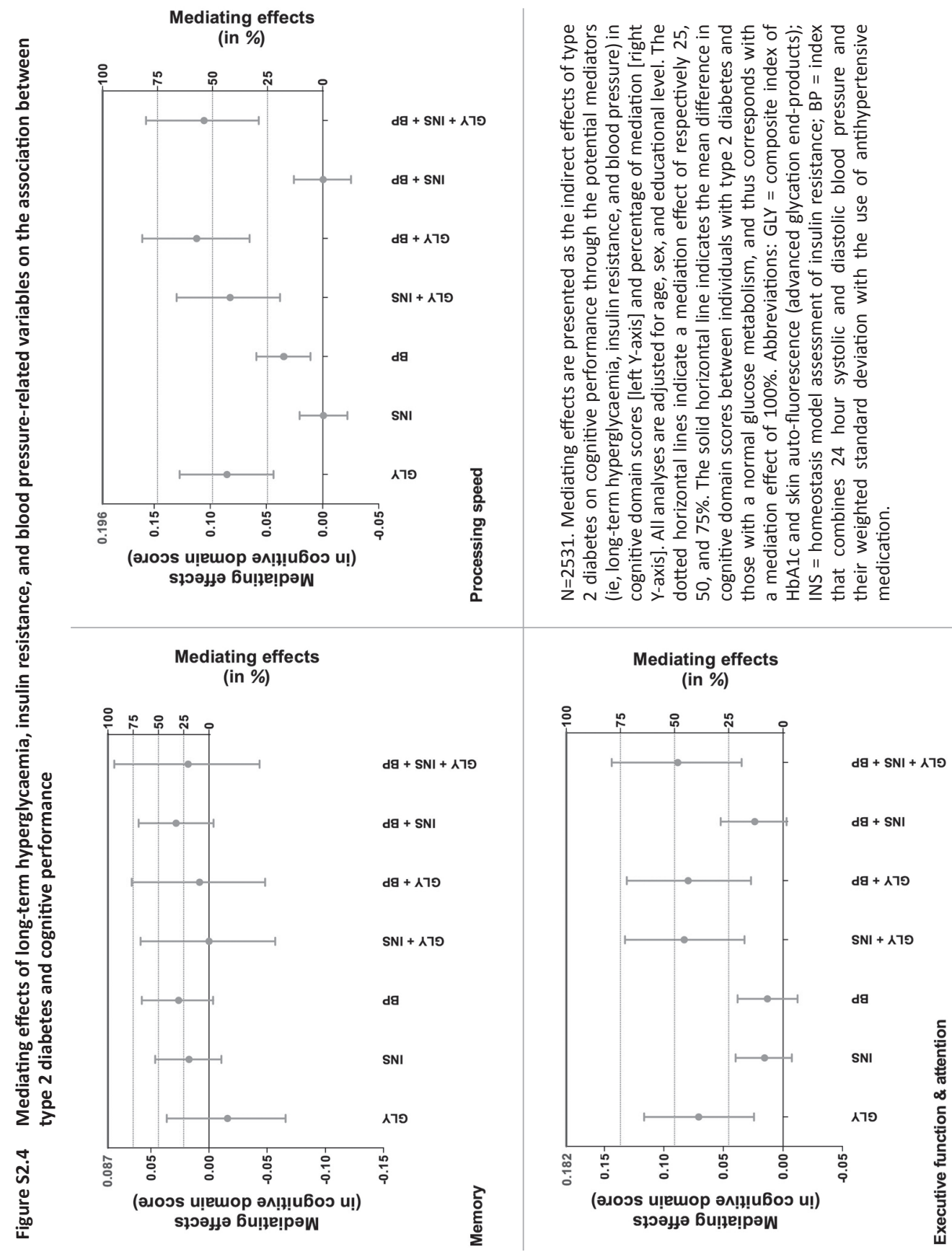

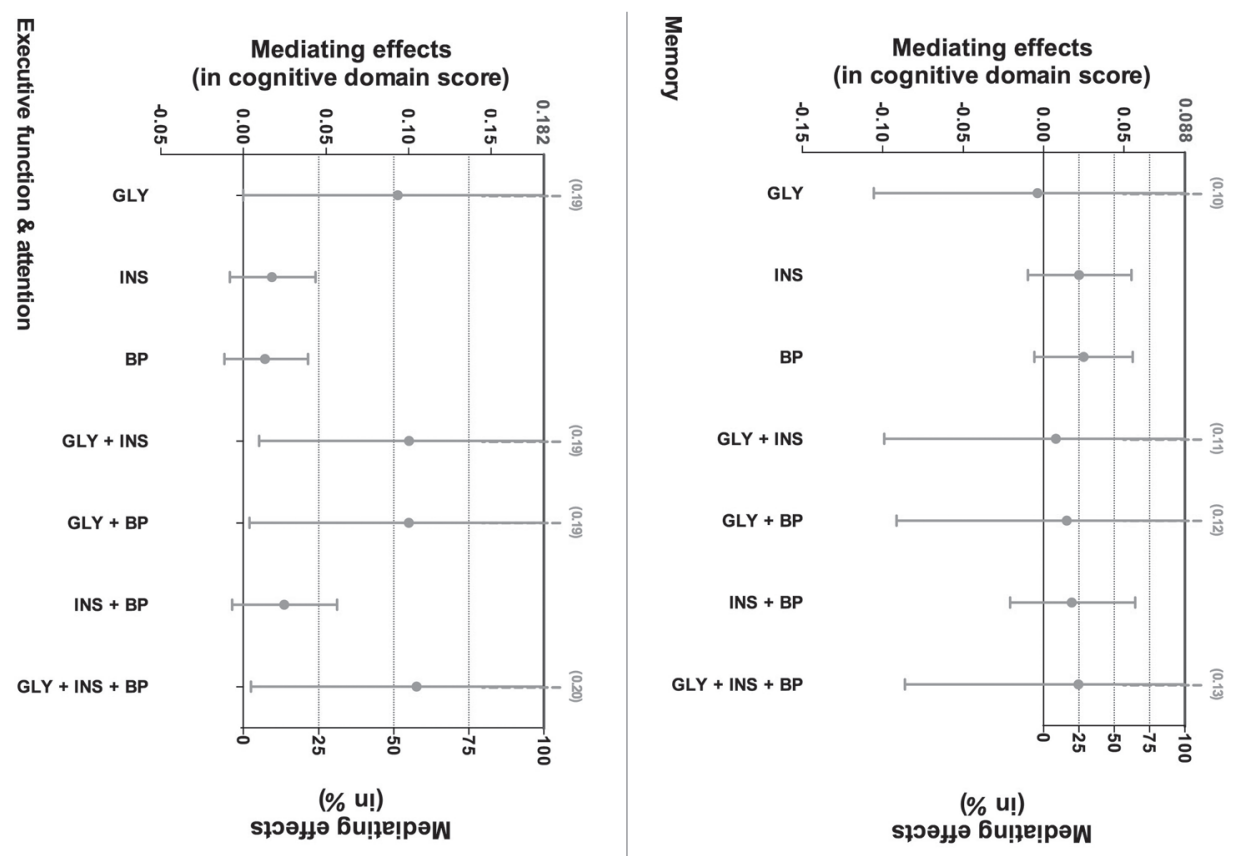

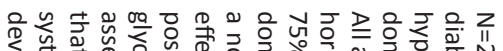

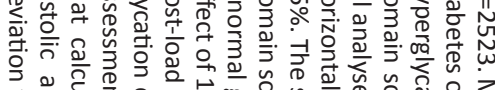

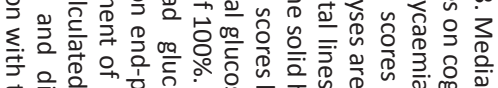

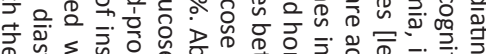

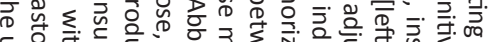

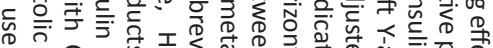

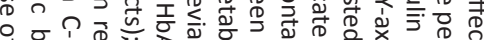

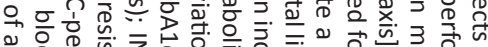

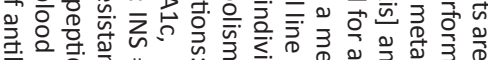

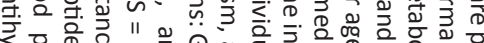

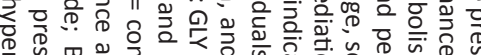

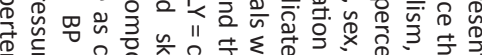

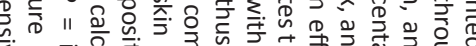

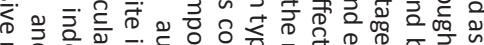
उ

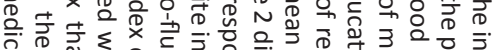

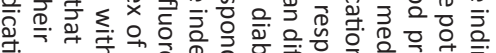

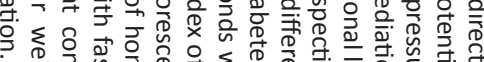

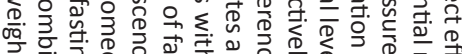

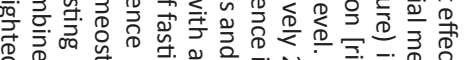

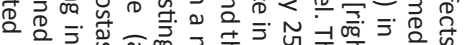

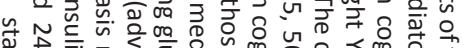

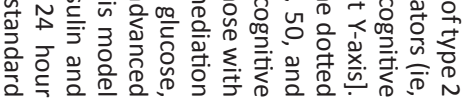

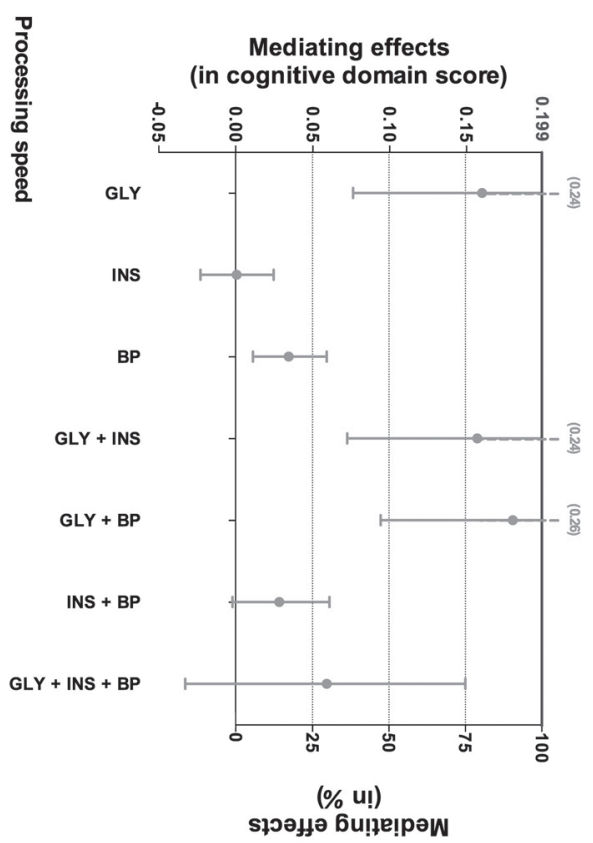




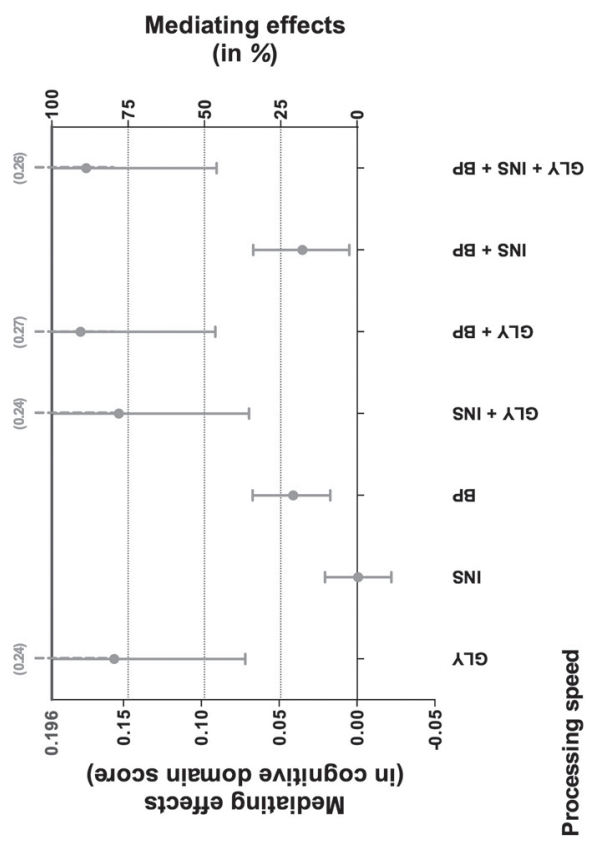

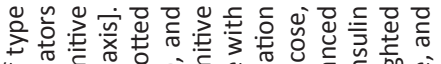

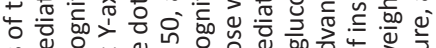

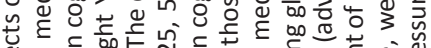

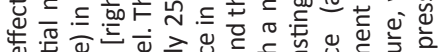

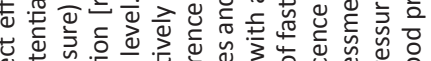

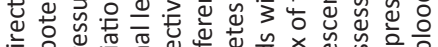

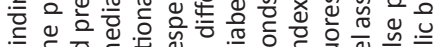

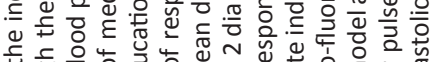

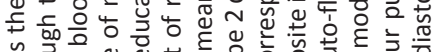

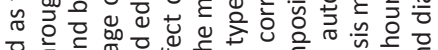

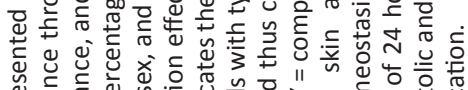

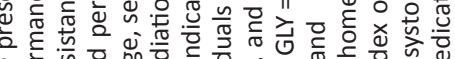

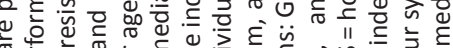

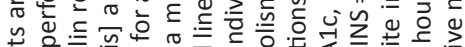

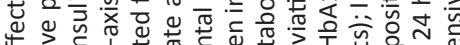

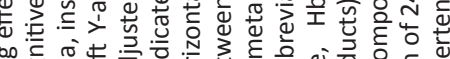

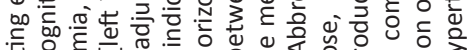

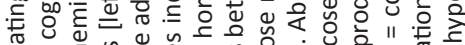
宗

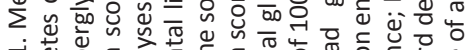

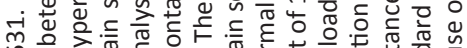
岇.

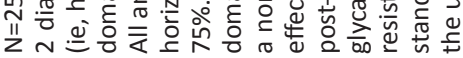

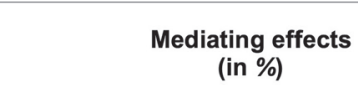

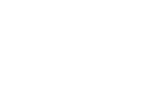

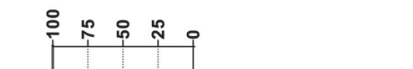

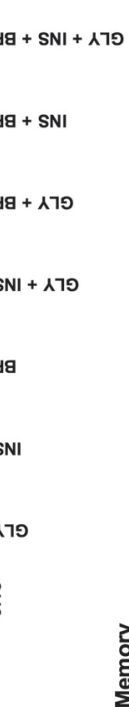

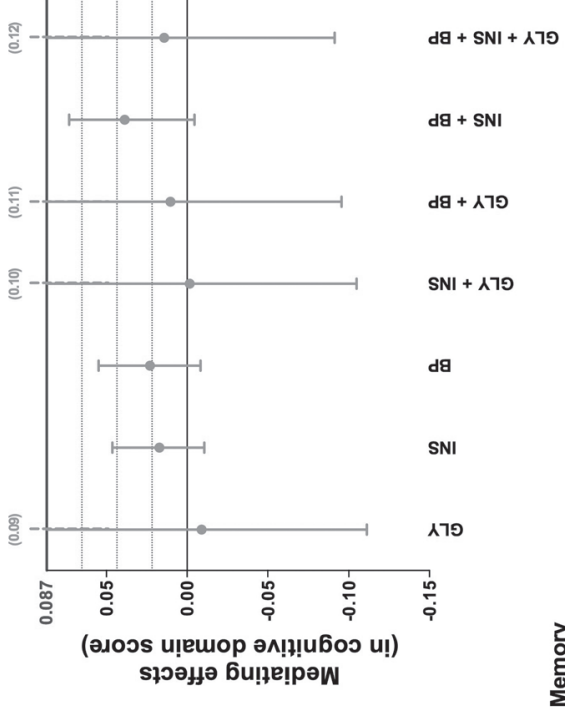

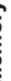

\section{Mediating effects
(in \%) \\ Mediating effects
(in \%)}

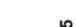

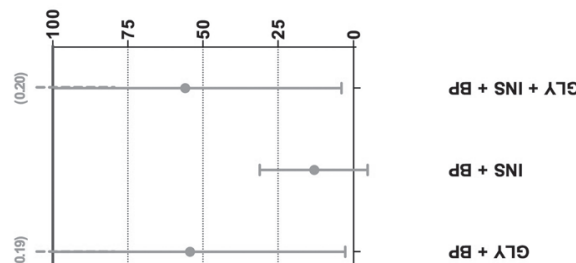

SNI + ᄉาว

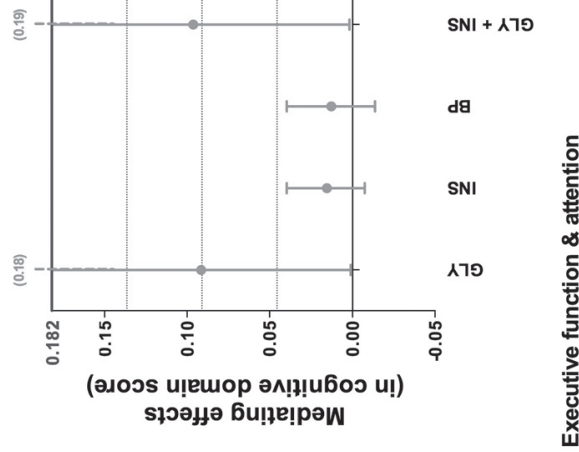


Figure S2.7 Mediating effects of hyperglycaemia, insulin metabolism, and blood pressurerelated variables on the association between type 2 diabetes and cognitive performance

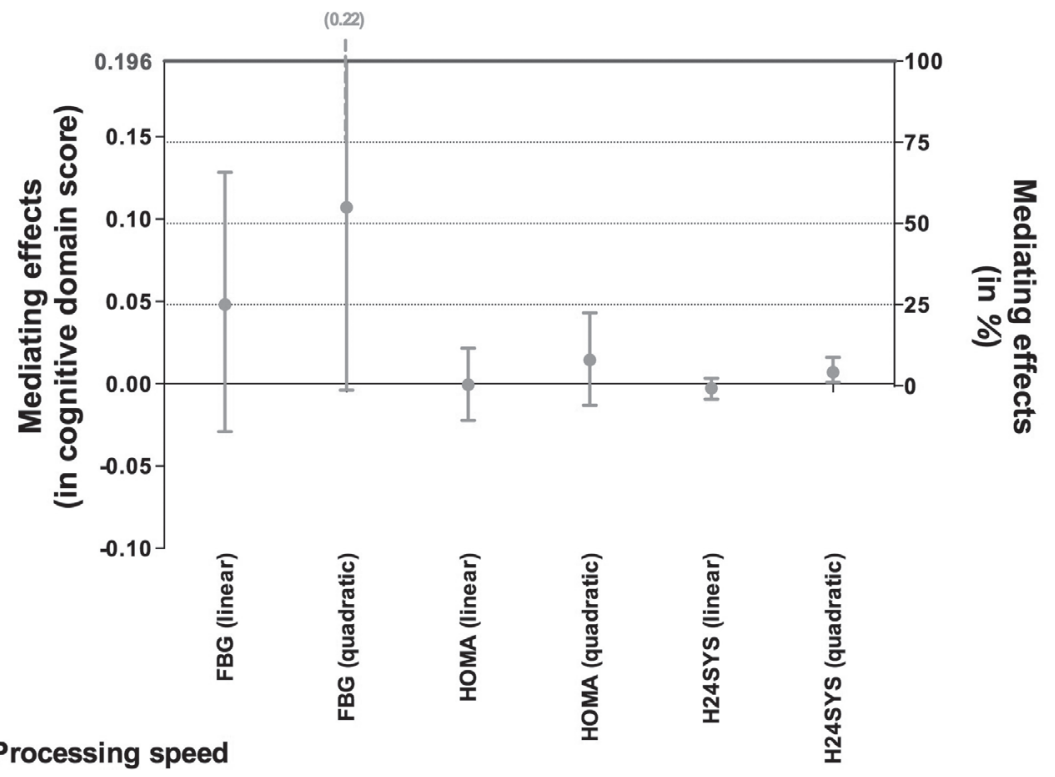

$\mathrm{N}=2531$. Mediating effects are presented as the indirect effects of type 2 diabetes on cognitive performance through the potential mediators in cognitive domain scores [left $Y$-axis] and percentage of mediation [right $\mathrm{Y}$-axis]. All analyses are adjusted for age, sex, and educational level. The dotted horizontal lines indicate a mediation effect of respectively 25,50 , and $75 \%$. The solid horizontal line indicates the mean difference in cognitive domain scores between individuals with type 2 diabetes and those with a normal glucose metabolism, and thus corresponds with a mediation effect of $100 \%$. Abbreviations: $F B G=$ fasting glucose; $\mathrm{HOMA}=$ homeostasis model assessment of insulin resistance; H24SYS = mean 24-hour systolic blood pressure. 
Mediating effects (in \%)

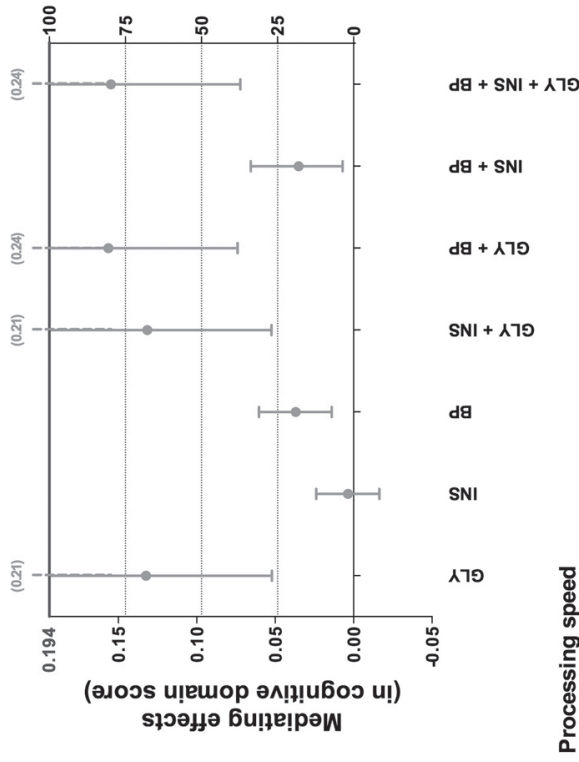

Mediating effects

(in \%)

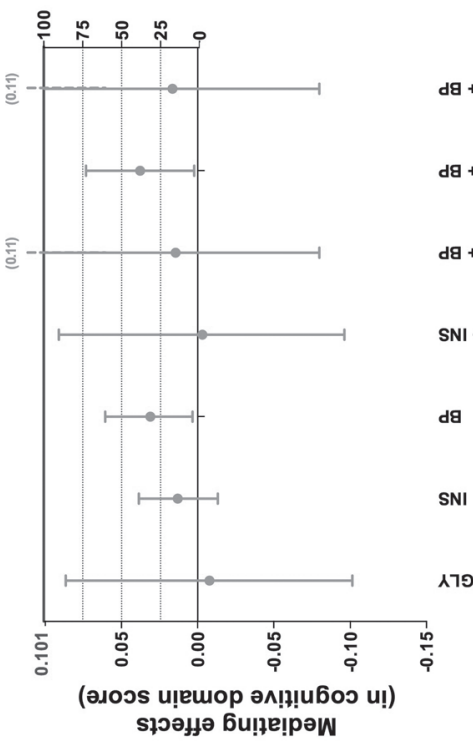

ํㅗㅇ

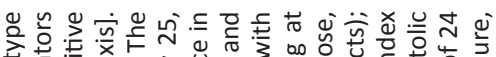

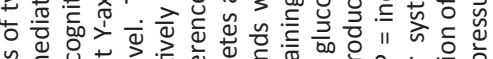

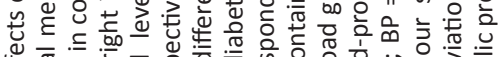

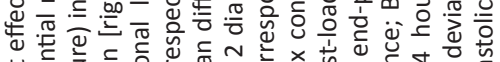

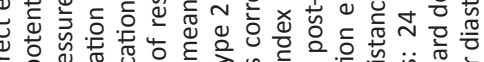

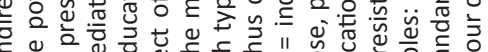

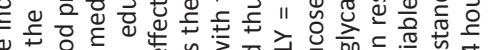

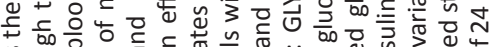

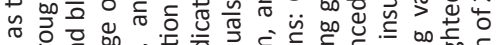

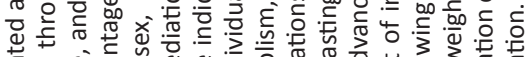
\& ه

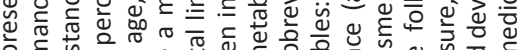

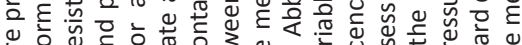

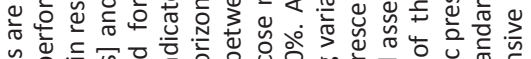

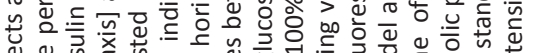

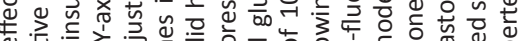

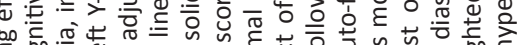
.

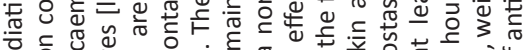

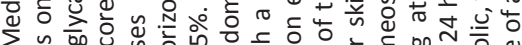

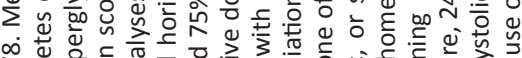

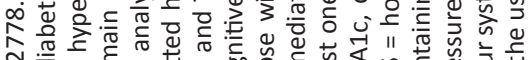

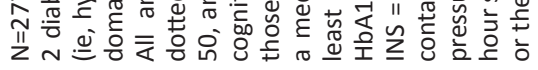

Mediating effects

(in \%)

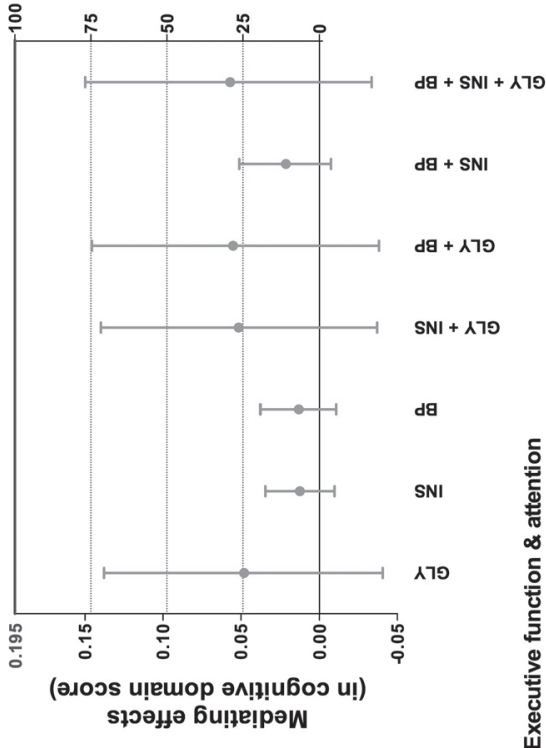


Exploring the aetiology of diabetes-associated worse cognitive performance 


\section{Chapter 3}

\section{CAROTID STIFFNESS IS ASSOCIATED WITH IMPAIRMENT OF COGNITIVE PERFORMANCE IN INDIVIDUALS WITH AND WITHOUT TYPE 2 DIABETES: THE MAASTRICHT STUDY}

Stefan L.C. Geijselaers, Simone J.S. Sep, Miranda T. Schram, Martin P.J. van Boxtel, Thomas T. van Sloten, Ronald M.A. Henry, Koen D. Reesink, Abraham A. Kroon, Annemarie Koster, Nicolaas C. Schaper, Pieter C. Dagnelie, Carla J.H. van der Kallen, Geert Jan Biessels, Coen D.A. Stehouwer 


\begin{abstract}
Background \& Aims

There is increasing evidence linking arterial (mainly aortic) stiffness and type 2 diabetes, a risk factor for arterial stiffness, to cognitive impairment and dementia. However, data on carotid stiffness, which may be especially relevant for cognitive performance, are scarce, and few studies have addressed the interplay between arterial stiffness, type 2 diabetes, and cognitive performance.
\end{abstract}

\title{
Methods
}

We studied individuals with $(n=197)$ and without $(n=528)$ type 2 diabetes who completed a neuropsychological test battery and underwent applanation tonometry and vascular ultrasound to evaluate aortic (ie, carotid-to-femoral pulse wave velocity) and carotid stiffness (ie, distensibility, compliance, and Young's elastic modulus). Linear regression analyses were performed and adjusted for demographics, vascular risk factors, and depression.

\section{Results}

Overall, our results showed that carotid, but not aortic, stiffness was associated with worse cognitive performance, primarily in the domains of processing speed (standardized regression coefficient for distensibility $-0.083, p=0.040$; compliance $-0.077, p=0.032$ ) and executive function and attention (distensibility $-0.133, p=0.001$; compliance $-0.090, p=0.015$; Young's elastic modulus $-0.081, p=0.027$ ). These associations did not differ by diabetes status. The differences in cognitive performance between individuals with and without type 2 diabetes (mean difference in domain scores relative to those without diabetes for free recall memory -0.23 , processing speed -0.19 , executive function and attention -0.23 ; all $p \leq 0.009$ and adjusted for demographics, traditional vascular risk factors, and depression) were not substantially altered after additional adjustment for carotid stiffness.

\section{Conclusion}

Our findings suggest that carotid stiffness is associated with cognitive performance in both individuals with and without diabetes, but does not mediate the relationship between type 2 diabetes and cognitive dysfunction. 


\section{Introduction}

In the ageing population, a rapidly increasing number of individuals will face cognitive deterioration later in life. It is estimated that in the United States, $36 \%$ of individuals aged over 70 are affected by some form of cognitive impairment. ${ }^{1}$ Yet, the pathophysiological mechanisms underlying age-related cognitive decline remain incompletely understood. Vascular factors are likely to be involved ${ }^{2}$ and may not be limited to local abnormalities of the cerebral vasculature, but may also include systemic vascular alterations such as arterial stiffening.

Arterial stiffening is part of the normal ageing process and results from a complex interplay between structural and cellular changes within the arterial wall. ${ }^{3}$ These changes include degenerative alterations, such as collagen accumulation, elastin fragmentation, and endothelial dysfunction. ${ }^{3}$ In theory, stiffening of (central) elastic arteries will hamper the natural cushioning function of the arterial system, thereby increasing pulsatility of pressure and flow in the brain vasculature. This increased pulsatile load can cause microvascular damage $e^{4,5}$ and may ultimately affect brain function.

Over the last decade, studies have collectively provided some evidence that arterial stiffness is negatively, but weakly, associated with cognitive performance. ${ }^{6}$ So far, the main focus has been on carotid-to-femoral pulse wave velocity (cfPWV), a measure of aortic stiffness that is considered the gold standard for the assessment of arterial stiffness. ${ }^{7}$ cfPWV, however, reflects stiffness of an arterial segment with mixed elastic and muscular properties, ${ }^{7}$ while stiffening of elastic and muscular arteries may differentially affect brain function, as the cushioning function of arteries diminishes from the most elastic to the more muscular ones. ${ }^{7}$ To date, studies evaluating the association of local stiffness of the predominantly elastic common carotid artery with cognitive performance are scarce and have yielded conflicting results..$^{8,9}$ Moreover, few studies have explored the interplay between arterial stiffness, cognitive performance, and type 2 diabetes. Type 2 diabetes is known to be associated with a variety of cognitive problems, ${ }^{10}$ ranging from subtle cognitive decrements to overt dementia, and a growing body of evidence also suggests ${ }^{11,12}$ that diabetes accelerates arterial stiffening. In addition, the ability of cerebral arteries to withstand increased pressure pulsatility may be decreased in individuals with diabetes. ${ }^{5}$ As such, it can be hypothesized that arterial stiffness is involved in diabetes-associated cognitive problems, a hypothesis that is corroborated by data linking arterial stiffness to other microvascular complications of type 2 diabetes (eg, nephropathy ${ }^{13}$ and retinopathy ${ }^{14}$ ). 
In view of the above, the aim of the present study was twofold. First, we investigated whether arterial stiffness was associated with cognitive performance in individuals with and without type 2 diabetes, and whether any such associations differed between aortic and carotid stiffness. Second, we investigated whether diabetes-associated impairment of cognitive performance could be explained by diabetes-associated increases in arterial stiffness, either aortic or carotid.

\section{Materials and methods}

\section{Study population}

In this study, we used data from The Maastricht Study, an ongoing observational prospective population-based cohort study that includes individuals aged 40-75 years living in the southern part of the Netherlands. The rationale and design have been described previously ${ }^{15}$ and are summarised in the Supplement. For the present study, cross-sectional data from the first 866 participants were used who completed the baseline survey between November 2010 and March 2012. Participants with type 1 diabetes $(n=4)$ or possible dementia (ie, those with a Mini Mental State Examination score $<24$, $\mathrm{n}=3$ ) were not eligible for the present study. From the eligible cohort, we additionally excluded a total of 134 (16\%) individuals because of missing data on one or more of the following variables: cognitive performance $(n=20)$, cfPWV $(n=41$; due to insufficient quality $(n=13)$ or logistical reasons $(n=28))$, measures of local carotid stiffness $(n=62$; due to technical failure $(n=5)$ or logistical reasons $(n=57))$, or one or more covariates ( $n=91$ ). Therefore, 725 individuals were available for analysis of aortic stiffness, cognitive performance, and type 2 diabetes and 711 for analysis of indices of carotid stiffness, cognitive performance, and type 2 diabetes. Characteristics of individuals with complete and incomplete data are shown in Table S3.1 (Supplement).

The Maastricht Study has been approved by the institutional medical ethical committee (NL31329.068.10) and the Netherlands Health Council under the Dutch "Law for Population Studies" (Permit 131088-105234-PG). All participants gave written informed consent.

\section{Cognitive assessment}

A concise battery $(30 \mathrm{~min}$ ) of neuropsychological tests was administered to assess cognitive performance. ${ }^{15}$ For conceptual clarity, and to reduce the number of cognitive outcomes, test results were divided into three cognitive domains (ie, free recall memory, processing speed, and executive function and attention), as specified in the Supplement. In short, free recall memory was evaluated using the Verbal Learning Test by averaging total immediate and delayed recall scores. The composite score for information 
processing speed was derived from the Stroop Colour Word Test Part I and II, the Concept Shifting Test Part A and B, and the Letter-Digit Substitution Test. Executive function and attention was assessed by the Stroop Colour Word Test Part III and the Concept Shifting Test Part C. Where necessary, individual test scores were inverted so that higher scores indicated better cognitive performance.

\section{Diabetes status}

A two-hour seven-sample oral glucose tolerance test was used to assess participants' glucose metabolism status, as previously described in more detail. ${ }^{15}$ Based on the WHO 2006 diagnostic criteria, ${ }^{16}$ individuals were classified as having normal glucose metabolism, impaired glucose metabolism, or diabetes. Participants were also considered to have type 2 diabetes if they used glucose-lowering medication without a prior diagnosis of type 1 diabetes.

\section{Measures of arterial stiffness}

Details on the measures of arterial stiffness and their reproducibility are provided in the Supplement. During the vascular assessment, brachial systolic, diastolic, and mean arterial pressure (MAP) were determined repeatedly with a 5-min interval, using an oscillometric device (Accutorr Plus, Datascope Inc., Montvale, NJ, USA), and the average of these measurements was calculated.

\section{Carotid-to-femoral pulse wave velocity}

Carotid-to-femoral pulse wave velocity (cfPWV) was determined according to recent guidelines ${ }^{17}$ with the use of applanation tonometry (SphygmoCor, Atcor Medical, Sydney, Australia), as specified in the Supplement.

\section{Indices of carotid stiffness}

Elastic properties of the left common carotid artery (at least $10 \mathrm{~mm}$ proximal to the carotid bulb) were obtained by using an ultrasound scanner equipped with a 7.5- $\mathrm{MHz}$ linear probe (MyLab 70, Esaote Europe B.V., Maastricht, the Netherlands). This setup enabled the measurement of diameter (D), distension (DS) and intima-media thickness (IMT) as described elsewhere ${ }^{18,19}$ and summarised in the Supplement. Combined with brachial pulse pressure (PP), these measures were used to calculate the following indices: ${ }^{20}$

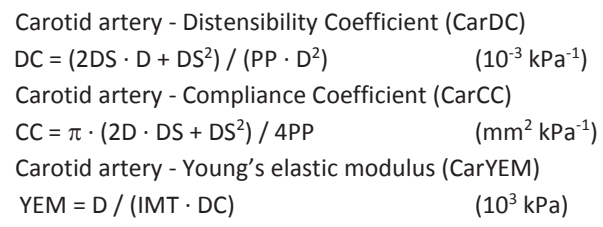


CarDC represents arterial stiffness, CarYEM the stiffness of the arterial wall material at operating pressure, and CarCC arterial buffering capacity. Please note that higher values of cfPWV and CarYEM, but lower values of CarDC and CarCC reflect greater arterial stiffness.

\section{Covariates}

A detailed description of the covariates is provided in the Supplement.

\section{Statistical analyses}

All analyses were performed using SPSS version 21.0 for Windows (IBM SPSS, IBM Corp, Armonk, NY, USA). Variables with a skewed distribution were transformed with the natural logarithm. A two-sided $p$-value $<0.05$ was considered statistically significant. No adjustments were made for multiple comparisons. ${ }^{21}$

Multiple linear regression analyses were used to explore the association between measures of arterial stiffness, cognitive performance, and type 2 diabetes. The associations were adjusted for potential confounders, including demographic characteristics (ie, age, sex, and educational level), haemodynamic factors (ie, MAP and heart rate obtained during vascular assessment), cardiovascular risk factors (ie, use of antihypertensive or lipid-modifying medication, total/high density lipoprotein-cholesterol ratio, triglyceride concentration, body mass index, smoking, alcohol consumption, and prior cardiovascular disease), and current depression where appropriate, as indicated in the tables. We deliberately chose to use slightly different models of adjustment across the different associations. Specifically, analyses with measures of arterial stiffness (eg, the association between arterial stiffness and cognitive performance) were adjusted for mean arterial pressure to disentangle blood pressure-dependent and -independent effects of arterial stiffness on cognitive performance. For this purpose, mean arterial pressure was chosen as it provides information on the non-pulsatile component of blood pressure. The association between type 2 diabetes and cognitive performance, on the other hand, was adjusted for (office-based) systolic and diastolic bood pressure to enhance the comparability of findings with other studies. Multiplicative terms (eg, CarDC * type 2 diabetes) were incorporated in the regression model to test interaction effects between type 2 diabetes and arterial stiffness on cognition with a confidence level of $90 \%$. The assumptions of linear regression were verified prior to all analyses.

The mediation effects of arterial stiffness on diabetes-associated impairment of cognitive performance were assessed by calculating the changes in regression coefficients of type 2 diabetes after inclusion of arterial stiffness indices in the model. Corresponding 95\% confidence intervals $(\mathrm{Cl})$ were estimated using SPSS macros provided by Preacher and 
Hayes $^{22}$ based on 5000 bootstrap iterations. A significant mediation effect was assumed when the $95 \% \mathrm{Cl}$ excluded zero. Mediation analyses were restricted to cognitive domains statistically significantly affected by the presence of diabetes and indices of arterial stiffness associated with cognitive performance.

Several sensitivity analyses were performed. First, analyses were repeated using indices of carotid stiffness that were calibrated with local carotid instead of brachial pulse pressure. Second, to examine the association between indices of carotid stiffness and cognitive performance in greater detail, we investigated whether the individual components of these indices (ie, carotid diameter, distension, and pulse pressure) were associated with cognitive performance. We also evaluated the correlation between indices of carotid stiffness and carotid-to-femoral pulse wave velocity with use of the Pearson correlation coefficient. Third, we tested for the presence of an association between, on the one hand, central pulse pressure and the augmentation index, and, on the other, cognitive performance. Although central pulse pressure and the augmentation index, which were both determined by radial applanation tonometry (SphygmoCor, Atcor Medical, Sydney, Australia) and pulse wave analysis, are considered indirect measures of arterial stiffness, they may provide additional information on wave reflections. ${ }^{7}$ Fourth, we evaluated the effect of adjustment for 24-hour mean arterial pressure and heart rate instead of adjustment for the average mean arterial pressure and heart rate obtained during vascular assessment (24-hour blood pressure data were only available in a subpopulation). Similarly, analysis of the association between type 2 diabetes and cognitive functioning were alternatively adjusted for mean arterial pressure, or for 24hour systolic and diastolic blood pressure, ${ }^{15}$ instead of office blood pressure. Fifth, we explored whether the association between type 2 diabetes and cognitive performance, or between type 2 diabetes and arterial stiffness, differed by hypertension status. ${ }^{15}$ Lastly, the potential confounding effects of physical activity (self-reported hours of moderate to vigorous physical activity per week) were explored.

\section{Results}

Table 3.1 shows the characteristics of the study population $(n=725$; mean age $60 \pm$ 8 years) with $(n=197)$ and without $(n=528)$ diabetes. Overall, individuals with type 2 diabetes were older, more often male, and had a more adverse cardiovascular risk profile. They were also more likely to have a history of cardiovascular disease, except for stroke, and were, in general, less educated than individuals without diabetes. The prevalence of depression did not differ between the two groups. 
Table 3.1 Characteristics of individuals with and without type 2 diabetes

\begin{tabular}{|c|c|c|c|c|}
\hline Clinical characteristics & $\begin{array}{c}\text { Total } \\
(n=725)\end{array}$ & $\begin{array}{l}\text { Type } 2 \text { diabetes } \\
(n=197)\end{array}$ & $\begin{array}{c}\text { No type } 2 \text { diabetes } \\
(n=528)\end{array}$ & p-value ${ }^{*}$ \\
\hline Age (years) & $60 \pm 8$ & $63 \pm 7$ & $58 \pm 8$ & $<0.001$ \\
\hline Male & 396 (54.6\%) & 137 (69.5\%) & 259 (49.1\%) & $<0.001$ \\
\hline Educational level & $113 / 302 / 310$ & $50 / 98 / 49$ & $63 / 204 / 261$ & $<0.001$ \\
\hline (low/middle/high) & $(15.6 \% / 41.7 \% / 42.8 \%)$ & $(25.4 \% / 49.7 \% / 24.9 \%)$ & (11.9\%/38.6\%/49.4\%) & \\
\hline $\mathrm{BMI}\left(\mathrm{kg} / \mathrm{m}^{2}\right)$ & $27.2 \pm 4.4$ & $29.6 \pm 4.7$ & $26.3 \pm 4.0$ & $<0.001$ \\
\hline Total cholesterol (mmol/L) & $5.2 \pm 1.2$ & $4.4 \pm 1.0$ & $5.5 \pm 1.1$ & $<0.001$ \\
\hline HDL-cholesterol (mmol/L) & $1.3 \pm 0.4$ & $1.1 \pm 0.3$ & $1.4 \pm 0.4$ & $<0.001$ \\
\hline Total/HDL cholesterol ratio & $4.2 \pm 1.2$ & $4.2 \pm 1.1$ & $4.2 \pm 1.3$ & 0.678 \\
\hline Triglycerides (mmol/L) & $1.2[0.9-1.8]$ & $1.6[1.1-2.3]$ & $1.1[0.8-1.6]$ & $<0.001$ \\
\hline Lipid-modifying medication & $255(35.2 \%)$ & $150(76.1 \%)$ & 105 (19.9\%) & $<0.001$ \\
\hline Brachial SBP $(\mathrm{mmHg})^{\dagger}$ & $128 \pm 14$ & $132 \pm 13$ & $127 \pm 14$ & $<0.001$ \\
\hline Brachial DBP $(\mathrm{mmHg})^{+}$ & $76 \pm 7$ & $76 \pm 7$ & $77 \pm 8$ & 0.385 \\
\hline Brachial MAP $(\mathrm{mmHg})^{+}$ & $97 \pm 10$ & $99 \pm 9$ & $97 \pm 10$ & 0.025 \\
\hline Brachial PP $(\mathrm{mmHg})^{+}$ & $52 \pm 10$ & $56 \pm 11$ & $50 \pm 10$ & $<0.001$ \\
\hline Heart rate $(\mathrm{bpm})^{+}$ & $63 \pm 9$ & $66 \pm 10$ & $62 \pm 8$ & $<0.001$ \\
\hline Hypertension $^{\ddagger}$ & $413(57.0 \%)$ & $171(86.8 \%)$ & 242 (45.8\%) & $<0.001$ \\
\hline Antihypertensive medication & $283(39.0 \%)$ & $140(71.1 \%)$ & $143(27.1 \%)$ & $<0.001$ \\
\hline eGFR $\left(\mathrm{ml} / \mathrm{min} / 1.73 \mathrm{~m}^{2}\right)$ & $89 \pm 15$ & $85 \pm 16$ & $90 \pm 14$ & $<0.001$ \\
\hline $\begin{array}{l}\text { Carotid-to-femoral pulse } \\
\text { wave velocity }(\mathrm{m} / \mathrm{s})\end{array}$ & $8.9 \pm 2.1$ & $9.8 \pm 2.4$ & $8.5 \pm 1.8$ & $<0.001$ \\
\hline $\begin{array}{l}\text { Alcohol consumption } \\
\text { (no/low/high) }\end{array}$ & $\begin{array}{c}120 / 381 / 224 \\
(16.6 \% / 52.6 \% / 30.9 \%)\end{array}$ & $\begin{array}{c}53 / 103 / 41 \\
(26.9 \% / 52.3 \% / 20.8 \%)\end{array}$ & $\begin{array}{c}67 / 278 / 183 \\
(12.7 \% / 52.7 \% / 34.7 \%)\end{array}$ & $<0.001$ \\
\hline $\begin{array}{l}\text { Smoking behaviour } \\
\text { (never/former/current) }\end{array}$ & $\begin{array}{c}226 / 384 / 115 \\
(31.2 \% / 53.0 \% / 15.9 \%)\end{array}$ & $\begin{array}{c}45 / 123 / 29 \\
(22.8 \% / 62.4 \% / 14.7 \%)\end{array}$ & $\begin{array}{c}181 / 261 / 86 \\
(34.3 \% / 49.4 \% / 16.3 \%)\end{array}$ & 0.004 \\
\hline Prior cardiovascular disease & $125(17.2 \%)$ & $56(28.4 \%)$ & $69(13.1 \%)$ & $<0.001$ \\
\hline \multirow[t]{2}{*}{ Current depression } & $28(3.9 \%)$ & $11(5.6 \%)$ & $17(3.2 \%)$ & 0.142 \\
\hline & $\begin{array}{c}\text { Total } \\
(n=711)\end{array}$ & $\begin{array}{l}\text { Type } 2 \text { diabetes } \\
(n=193)\end{array}$ & $\begin{array}{c}\text { No type } 2 \text { diabetes } \\
(n=518)\end{array}$ & p-value* \\
\hline $\begin{array}{l}\text { Carotid artery - distensibility } \\
\text { coefficient }\left(10^{-3} / \mathrm{kPa}\right)\end{array}$ & $13.7 \pm 4.8$ & $12.2 \pm 4.1$ & $14.2 \pm 4.9$ & $<0.001$ \\
\hline $\begin{array}{l}\text { Carotid artery - compliance } \\
\text { coefficient }\left(\mathrm{mm}^{2} / \mathrm{kPa}\right)\end{array}$ & $0.65 \pm 0.26$ & $0.63 \pm 0.24$ & $0.66 \pm 0.26$ & 0.206 \\
\hline $\begin{array}{l}\text { Carotid artery - Young's } \\
\text { elastic modulus }\left(10^{3} / \mathrm{kPa}\right)\end{array}$ & $0.78 \pm 0.36$ & $0.86 \pm 0.45$ & $0.75 \pm 0.31$ & $<0.001$ \\
\hline Carotid PP $(\mathrm{mmHg})^{\S}$ & $51 \pm 14$ & $56 \pm 15$ & $49 \pm 13$ & $<0.001$ \\
\hline
\end{tabular}

Data are presented as mean $\pm S D$, median [IQR], or $n(\%) .{ }^{*} \mathrm{P}$-value for difference between individuals with and without type 2 diabetes, based on independent samples t-tests for continuous variables and Chi-square tests for categorical variables. ${ }^{+}$Obtained from blood pressure measurements every 5 minutes during vascular assessment. ${ }^{*}$ Hypertension was defined as a systolic blood pressure $\geq 140 \mathrm{mmHg}$ (based on office blood pressure measurements ${ }^{15}$ ), a diastolic blood pressure $\geq 90 \mathrm{mmHg}$, and/or current use of antihypertensive medication. ${ }^{5} \mathrm{~N}=708$, of which $\mathrm{n}=191$ with type 2 diabetes. Abbreviations: BMI = Body Mass Index; HDL = high density lipoprotein; SBP = systolic blood pressure; $\mathrm{DBP}=$ diastolic blood pressure; $\mathrm{MAP}=$ mean arterial pressure; PP = pulse pressure; eGFR = estimated glomerular filtration rate. 
Of the 528 individuals without diabetes, 124 (23\%) were classified as having impaired glucose metabolism. Age- and sex-adjusted cognitive performance did not differ between individuals with normal and impaired glucose metabolism (data not shown). We therefore did not analyse these groups separately.

\section{Arterial stiffness and cognitive performance}

Table 3.2 shows the association between measures of arterial stiffness and cognitive performance. After adjustment for age, sex, and educational level, higher cfPWV tended to be associated with impaired free recall memory (standardised regression coefficient $\beta[95 \% \mathrm{Cl}]-0.063$ [-0.134 to 0.007$]$, model 1$)$ and information processing speed $(-0.058$ [-0.129 to 0.013$]$, model 1 ), but these associations did not reach statistical significance. In the fully adjusted models (model 3), cfPWV was not associated with cognitive performance.

Lower carotid distensibility (CarDC) and compliance (CarCC) were associated with worse information processing speed and executive function and attention in fully adjusted models. Higher Young's elastic modulus (CarYEM) was only statistically significantly associated with lower performance in executive function and attention ( $\beta-0.081[-0.152$ to -0.009 ], model 3). Indices of carotid stiffness were not related to free recall memory (models 1-3). Calculations using the regression coefficients of age and indices of carotid stiffness (from the fully adjusted model, model 3) showed that one standard deviation increase in carotid stiffness was comparable with 1 to 2 years of ageing in the domain of processing speed and 2 to 4 years of ageing in the domain of executive function and attention (data not shown).

The associations between measures of arterial stiffness and cognitive functioning did not statistically significantly differ between individuals with and without diabetes ( $p$-values for interaction $\geq 0.13$ ).

\section{Type 2 diabetes and cognitive performance}

Table 3.3 shows the association between type 2 diabetes and cognitive performance. After adjustment for age, sex, and educational level, the presence of type 2 diabetes was associated with lower performance in all cognitive domains assessed (mean difference in cognitive domain scores for free recall memory [95\% Cl] $-0.23[-0.37$ to -0.09$]$; processing speed -0.16 [-0.28 to -0.04$]$; executive function and attention -0.26 [-0.39 to -0.14]; model 1). Additional adjustments for multiple cardiovascular risk factors and current depression did not materially alter these associations (model 2). Raw cognitive test results for individuals with and without type 2 diabetes are presented in Table S3.2 (Supplement). 
Table 3.2 Association between measures of arterial stiffness and cognitive performance

\begin{tabular}{|c|c|c|c|}
\hline Model & $\begin{array}{c}\text { Free recall } \\
\text { memory }\end{array}$ & Processing speed & $\begin{array}{c}\text { Executive function } \& \\
\text { attention }\end{array}$ \\
\hline \multicolumn{4}{|c|}{ Carotid-to-femoral pulse wave velocity } \\
\hline Model 1 & $\begin{array}{c}-0.063 \\
(-0.134 \text { to } 0.007)\end{array}$ & $\begin{array}{c}-0.058 \\
(-0.129 \text { to } 0.013)\end{array}$ & $\begin{array}{c}0.008 \\
(-0.065 \text { to } 0.082)\end{array}$ \\
\hline Model 2 & $\begin{array}{c}-0.057 \\
(-0.136 \text { to } 0.021)\end{array}$ & $\begin{array}{c}-0.086^{*} \\
(-0.166 \text { to }-0.007)\end{array}$ & $\begin{array}{c}0.020 \\
(-0.062 \text { to } 0.102)\end{array}$ \\
\hline Model 3 & $\begin{array}{c}-0.028 \\
(-0.108 \text { to } 0.051)\end{array}$ & $\begin{array}{c}-0.067 \\
(-0.147 \text { to } 0.014)\end{array}$ & $\begin{array}{c}0.047 \\
(-0.037 \text { to } 0.130)\end{array}$ \\
\hline \multicolumn{4}{|c|}{ Carotid artery - distensibility coefficient } \\
\hline Model 1 & $\begin{array}{c}-0.032 \\
(-0.104 \text { to } 0.040)\end{array}$ & $\begin{array}{c}-0.060 \\
(-0.133 \text { to } 0.012)\end{array}$ & $\begin{array}{c}-0.125^{*} \\
(-0.200 \text { to }-0.051)\end{array}$ \\
\hline Model 2 & $\begin{array}{c}-0.022 \\
(-0.101 \text { to } 0.057)\end{array}$ & $\begin{array}{c}-0.087^{*} \\
(-0.166 \text { to }-0.008)\end{array}$ & $\begin{array}{c}-0.143^{*} \\
(-0.224 \text { to }-0.061)\end{array}$ \\
\hline Model 3 & $\begin{array}{c}-0.007 \\
(-0.085 \text { to } 0.071)\end{array}$ & $\begin{array}{c}-0.083^{*} \\
(-0.162 \text { to }-0.004)\end{array}$ & $\begin{array}{c}-0.133^{*} \\
(-0.215 \text { to }-0.052)\end{array}$ \\
\hline \multicolumn{4}{|c|}{ Carotid artery - compliance coefficient } \\
\hline Model 1 & $\begin{array}{c}0.003 \\
(-0.065 \text { to } 0.071)\end{array}$ & $\begin{array}{c}-0.061 \\
(-0.130 \text { to } 0.007)\end{array}$ & $\begin{array}{c}-0.088^{*} \\
(-0.159 \text { to }-0.018)\end{array}$ \\
\hline Model 2 & $\begin{array}{c}0.012 \\
(-0.058 \text { to } 0.082)\end{array}$ & $\begin{array}{c}-0.073^{*} \\
(-0.144 \text { to }-0.003)\end{array}$ & $\begin{array}{c}-0.091^{*} \\
(-0.164 \text { to }-0.018)\end{array}$ \\
\hline Model 3 & $\begin{array}{c}0.007 \\
(-0.063 \text { to } 0.076)\end{array}$ & $\begin{array}{c}-0.077^{*} \\
(-0.147 \text { to }-0.007)\end{array}$ & $\begin{array}{c}-0.090^{*} \\
(-0.163 \text { to }-0.018)\end{array}$ \\
\hline \multicolumn{4}{|c|}{ Carotid artery - Young's elastic modulus } \\
\hline Model 1 & $\begin{array}{c}-0.011 \\
(-0.077 \text { to } 0.054)\end{array}$ & $\begin{array}{c}-0.046 \\
(-0.112 \text { to } 0.020)\end{array}$ & $\begin{array}{c}-0.088^{*} \\
(-0.156 \text { to }-0.020)\end{array}$ \\
\hline Model 2 & $\begin{array}{c}-0.003 \\
(-0.072 \text { to } 0.065)\end{array}$ & $\begin{array}{c}-0.059 \\
(-0.128 \text { to } 0.010)\end{array}$ & $\begin{array}{c}-0.092^{*} \\
(-0.163 \text { to }-0.021)\end{array}$ \\
\hline Model 3 & $\begin{array}{c}0.008 \\
(-0.060 \text { to } 0.076)\end{array}$ & $\begin{array}{c}-0.054 \\
(-0.123 \text { to } 0.015)\end{array}$ & $\begin{array}{c}-0.081^{*} \\
(-0.152 \text { to }-0.009)\end{array}$ \\
\hline
\end{tabular}

Data are presented as standardised regression coefficient $(95 \% \mathrm{Cl}) .{ }^{*} \mathrm{p}<005$. Regression coefficients indicate the change in cognitive performance per SD increase in carotid-to-femoral pulse wave velocity or carotid Young's elastic modulus and per SD decrease in carotid distensibility or compliance; in other words, a negative regression coefficient means that greater arterial stiffness is associated with worse cognitive performance. Model 1: adjusted for age, sex, and educational level; Model 2: additional adjustments for mean arterial pressure and heart rate (the latter only for analyses of carotid-to-femoral pulse wave velocity) ; Model 3: additional adjustments for presence of type 2 diabetes, body mass index, total/high density lipoproteincholesterol ratio, triglycerides, use of lipid-modifying medication, use of antihypertensive medication, estimated glomerular filtration rate, smoking behaviour, alcohol consumption, current depression, history of cardiovascular disease(s). Abbreviations: $\mathrm{Cl}=$ confidence interval; $\mathrm{SD}=$ standard deviation. 
Table 3.3 Mean difference in cognitive domain scores between individuals with and without type 2 diabetes

\begin{tabular}{|c|c|c|c|}
\hline & Free recall memory & Processing speed & $\begin{array}{c}\text { Executive function \& } \\
\text { attention }\end{array}$ \\
\hline \multicolumn{4}{|l|}{ Model } \\
\hline Model 1 & $\begin{array}{c}-0.229^{*} \\
(-0.371 \text { to }-0.088)\end{array}$ & $\begin{array}{c}-0.160^{*} \\
(-0.278 \text { to }-0.042)\end{array}$ & $\begin{array}{c}-0.260^{*} \\
(-0.385 \text { to }-0.136)\end{array}$ \\
\hline Model 2 & $\begin{array}{c}-0.230^{*} \\
(-0.397 \text { to }-0.064)\end{array}$ & $\begin{array}{c}-0.187^{*} \\
(-0.327 \text { to }-0.047)\end{array}$ & $\begin{array}{c}-0.231^{*} \\
(-0.380 \text { to }-0.081)\end{array}$ \\
\hline
\end{tabular}

Data are presented as unstandardised regression coefficient $(95 \% \mathrm{Cl}) .{ }^{*} \mathrm{p}<005$. Individuals without diabetes were used as the reference group. Regression coefficients indicate the mean difference in cognitive domain scores between individuals with and without type 2 diabetes. Model 1: adjusted for sex, age, and educational level; Model 2: additional adjustments for body mass index, total/high density lipoprotein-cholesterol ratio, triglycerides, lipid-modifying medication, systolic blood pressure, diastolic blood pressure, antihypertensive medication, estimated glomerular filtration rate, smoking behaviour, alcohol consumption, current depression, and history of cardiovascular disease(s). Abbreviations: $\mathrm{Cl}=$ confidence interval.

Table 3.4 Mean difference in indices of arterial stiffness between individuals with and without type 2 diabetes

\begin{tabular}{|c|c|c|c|c|}
\hline Model & $\begin{array}{l}\text { Carotid-to-femoral } \\
\text { pulse wave velocity } \\
(\mathrm{m} / \mathrm{s})\end{array}$ & $\begin{array}{l}\text { Carotid artery - } \\
\text { distensibility } \\
\text { coefficient } \\
\left(10^{-3} / \mathrm{kPa}\right)\end{array}$ & $\begin{array}{c}\text { Carotid artery- } \\
\text { compliance } \\
\text { coefficient } \\
\left(\mathrm{mm}^{2} / \mathrm{kPa}\right)\end{array}$ & $\begin{array}{c}\text { Carotid artery- } \\
\text { Young's elastic } \\
\text { modulus } \\
\left(10^{3} / \mathrm{kPa}\right)\end{array}$ \\
\hline Model 1 & $\begin{array}{c}0.618^{*} \\
(0.309 \text { to } 0.927)\end{array}$ & $\begin{array}{c}-0.710 \\
(-1.437 \text { to } 0.018)\end{array}$ & $\begin{array}{c}-0.017 \\
(-0.059 \text { to } 0.024)\end{array}$ & $\begin{array}{c}0.046 \\
(-0.013 \text { to } 0.106)\end{array}$ \\
\hline Model 2 & $\begin{array}{c}0.490^{*} \\
(0.207 \text { to } 0.773)\end{array}$ & $\begin{array}{c}-0.630 \\
(-1.294 \text { to } 0.035)\end{array}$ & $\begin{array}{c}-0.015 \\
(-0.055 \text { to } 0.026)\end{array}$ & $\begin{array}{c}0.042 \\
(-0.015 \text { to } 0.099)\end{array}$ \\
\hline Model 3 & $\begin{array}{c}0.450^{*} \\
(0.117 \text { to } 0.783)\end{array}$ & $\begin{array}{c}-0.309 \\
(-1.149 \text { to } 0.479)\end{array}$ & $\begin{array}{c}-0.033 \\
(-0.080 \text { to } 0.015)\end{array}$ & $\begin{array}{c}0.018 \\
(-0.049 \text { to } 0.086)\end{array}$ \\
\hline
\end{tabular}

Data are presented as unstandardised regression coefficient $(95 \% \mathrm{Cl}) .{ }^{*} \mathrm{p}<005$. Individuals without diabetes were used as the reference group. Regression coefficients indicate the mean difference in indices of arterial stiffness between individuals with and without type 2 diabetes. Model 1: adjusted for sex and age; Model 2: additional adjustments for mean arterial pressure and heart rate (the latter only for analyses of carotidto-femoral pulse wave velocity); Model 3: additional adjustments for body mass index, total/high density lipoprotein-cholesterol ratio, triglycerides, use of lipid-modifying medication, use of antihypertensive medication, smoking behaviour, estimated glomerular filtration rate, history of cardiovascular disease(s). Abbreviations: $\mathrm{Cl}=$ confidence interval. 
Type 2 diabetes and arterial stiffness

Table 3.4 shows the associations between type 2 diabetes and measures of arterial stiffness. After adjustment for age and sex, individuals with type 2 diabetes had higher cfPWV (mean difference [95\% $\mathrm{Cl}] 0.62[0.31$ to 0.93$] \mathrm{m} / \mathrm{s}$ ) and tended to have lower, albeit not statistically significant, CarDC (-0.71 [-1.44 to 0.02$] 10-3 / \mathrm{kPa})$ than individuals without diabetes (model 1). The association between diabetes status and cfPWV weakened, but remained significant after further adjustment for haemodynamic $(0.49$ [0.21 to 0.77 ] m/s, model 2 ) and other cardiovascular risk factors $(0.45$ [0.12 to 0.78$] \mathrm{m} / \mathrm{s}$, model 3 ), whereas the trend with CarDC was no longer observed in the fully adjusted model (-0.31 [-1.15 to 0.48] 10-3/kPa, model 3). The age- and sex-adjusted differences in CarCC en CarYEM between individuals with and without type 2 diabetes were not statistically significant (model 1 ).

\section{Mediation of the association between type 2 diabetes and cognitive performance by arterial stiffness}

The mediation analyses were restricted to the cognitive domains observed to be associated with indices of carotid stiffness: information processing speed and executive function and attention. Differences in cognitive performance between individuals with and without type 2 diabetes, adjusted for age, sex, and educational level, were only slightly diminished after additional adjustment for CarDC, CarCC, or CarYEM (maximum change in regression coefficient (ie, change in mean differences in cognitive domain scores) was -0.013 , with a bootstrapped $95 \% \mathrm{Cl}$ of [-0.0384 to -0.0012$]$ ). In fully adjusted models there were no statistically significant mediation effects of carotid stiffness indices on the association of type 2 diabetes with cognitive performance (data not shown). Likewise, no mediation was found when exploring the combined mediating effects of aortic and carotid stiffness (eg, cfPWV and CarDC, data not shown).

\section{Sensitivity analyses}

When carotid stiffness was calibrated with carotid instead of brachial pulse pressure (PP), the results were largely similar to those obtained with use of brachial PP (Tables S3.3 and S3.4, Supplement), except for the association between type 2 diabetes and carotid stiffness, which appeared slightly stronger (Table S3.3, Online Supplement). Specifically, after adjustment for demographics and haemodynamic factors, individuals with diabetes had a statistically significantly lower CarDC than those without (mean difference [95\% Cl] -1.04 [-1.94 to -0.15$]$, model 1 , and -0.94 [-1.71 to -0.16$] 10-3 / \mathrm{kPa}$, model 2, respectively), but this association was not independent of other traditional cardiovascular risk factors (-0.39 [-1.31 to 0.53$] 10-3 / \mathrm{kPa}$, model 3 ). Results of mediation analyses using indices of carotid stiffness that were calibrated with carotid PP were similar to those obtained with brachial PP (data not shown). 
A more detailed analysis of the association between indices of carotid stiffness and cognitive performance showed that only carotid distension was statistically significantly associated with cognitive performance, whereas carotid diameter and PP, as well as brachial PP, were not (Table S3.5, Supplement). In addition, neither the augmentation index nor central PP appeared to be associated with cognitive performance (Table S3.6, Supplement). Mediation analyses performed in the fully adjusted models showed that none of these individual variables had any statistically significant mediating effect on the association between type 2 diabetes and cognitive performance, nor when they were combined with aortic stiffness (data not shown). Correlation analyses revealed that indices of carotid stiffness were only weakly to moderately correlated with cfPWV (Table S3.7, Supplement).

Associations between type 2 diabetes and cognitive performance, or between type 2 diabetes and arterial stiffness, may differ between individuals with and without hypertension. However, we found no such interaction, except for the association of diabetes with impairments in executive function and attention, which was present in individuals with hypertension (mean difference in cognitive domain score $[95 \% \mathrm{Cl}]$ -0.27 [-0.45 to -0.10$]$ ), but not in individuals without hypertension (-0.06 [-0.39 to 0.27], fully adjusted models). Additional mediation analyses performed in the subgroup of individuals with hypertension showed that this association also was not mediated by carotid stiffness (data not shown).

Alternative adjustment for 24-hour blood pressure and additional adjustment for physical activity did not materially alter our results (see Supplement).

\section{Discussion}

The results of this study show that greater carotid, but not aortic, arterial stiffness is associated with worse cognitive performance, both in individuals with and without type 2 diabetes. However, indices of carotid stiffness do not mediate the relationship between type 2 diabetes and worse cognitive performance.

Although interrelated, aortic and carotid stiffness are not interchangeable. ${ }^{23}$ Aortic stiffness, as reflected by cfPWV, involves a segment of the arterial tree with both elastic and muscular properties, ${ }^{7}$ whereas the common carotid artery is a predominantly elastic vessel. Muscular arteries are characterized by a higher collagen to elastin ratio than elastic arteries. ${ }^{24}$ Because the cushioning function progressively diminishes from the most elastic to the more muscular arteries, ${ }^{7}$ pulsatile load on the brain is particularly likely to increase with stiffening of elastic arteries. ${ }^{25}$ As such, differences in structural properties 
may explain our finding that carotid, but not aortic, arterial stiffness was negatively associated with cognitive performance. This is further supported by our observation that stiffness of the femoral artery, an artery of the muscular type, was not associated with cognitive functioning (data not shown). Data from the ARIC (Atherosclerosis Risk In Communities) study have previously indicated that carotid stiffness is more strongly related to cerebrovascular disease (ie, incident ischemic stroke), ${ }^{26}$ whereas femoral stiffness has been suggested to be more strongly related to coronary heart disease. ${ }^{25}$

To our knowledge, only three other studies have investigated the relationship between carotid stiffness and cognitive performance, with conflicting results. In line with our findings, findings of two studies in middle-aged ( $n=58$, mean age $53 \pm 1$ years) ${ }^{9}$ and late middle-aged $(n=308 \text {, mean age } 63 \pm 6 \text { years })^{27}$ adults showed that carotid stiffness was associated with decrements in executive function and overall cognition, ${ }^{9,}{ }^{27}$ while cfPWV was not consistently associated with cognitive performance in these studies. In older individuals from the Rotterdam Study ( $n=3714$, mean age at baseline $72 \pm 7$ years), however, no association, neither cross-sectionally nor longitudinally, was observed between CarDC and (changes in) cognitive test scores after adjustment for cardiovascular risk factors. ${ }^{8}$ Whether these differences in study results can be explained by differences in age is at present unknown. Aortic stiffness has been studied more frequently in relation to cognition. Our finding that cfPWV was not related to cognitive performance seems to be in contrast with most, ${ }^{4,8,9,28-41}$ but not all, ${ }^{42,43}$ previous studies. Part of this discrepancy may be explained by the fact that previous studies were often conduced in elderly subjects (ie, mean age over 70), 8, 28-33, 39 or individuals with (subjective) memory loss, $28-30,37$ with larger between-individual variation in cognitive performance, and frequently did not control for potential confounders such as MAP, $9,28-31,33,36,37$ or depressive symptoms. . $^{8,28-30,36,37,39}$ Nonetheless, this does not explain why our findings of cfPWV contrast with those of comparable population-based cohort studies, ${ }^{34,38,40,41}$ although this might be due to use of varying cognitive tests focusing on different aspects of cognitive performance. Given the current literature, we can thus not conclude from our data that aortic stiffness is unrelated to cognitive performance, but our data do suggest that carotid stiffness is more strongly associated with impairments in cognitive performance.

Although further studies are needed to fully elucidate the mechanisms that relate carotid stiffness to cognitive performance, it can be speculated that an impaired cushioning function of elastic arteries contributes to the development and progression of cerebral small vessel disease. The potential role of the arterial cushioning function in linking carotid stiffness to cognitive performance is illustrated by our observation that carotid distension, but not carotid diameter or PP, was associated with cognitive performance. 
The consequential increase in pulsatile load on the brain is likely to induce alterations in the cerebral microcirculation (eg, hypertrophic remodelling and rarefaction of small arteries) which, in turn, can lead to (chronic) cerebral ischaemia. ${ }^{44}$ In this regard, our finding that neither PP nor the augmentation index was associated with cognitive performance may suggest that, as proposed previously, ${ }^{4}$ transmission of excessive flow rather than pressure pulsatility has a negative impact on the cerebral microcirculation. It could, however, also reflect the fact that PP and the augmentation index are indirect measures of arterial stiffness that depend on multiple factors, including the duration and pattern of ventricular contraction. ${ }^{7}$

The idea that cerebral small vessel disease may link carotid stiffness to cognitive performance is supported by recent studies showing that carotid stiffness is associated with higher volumes of white matter hyperintensities ${ }^{45,46}$ and non-lacunar infarcts, ${ }^{46}$ independently of other vascular factors. Carotid stiffness does, however, not predict the progression of cerebral small vessel disease ${ }^{46}$ which is inconsistent with small vessel disease being an important factor contributing to the observed decrements in cognitive performance with carotid stiffness. Alternatively, carotid stiffness may affect carotid baroreceptor sensitivity ${ }^{47}$ or could simply reflect structural changes in cerebral arteries including increased collagen deposition, calcification, and fibrosis.

It has been suggested that vascular factors, ${ }^{10}$ such as arterial stiffness, may also be involved in the pathogenesis of diabetes-associated cognitive problems. The profile and magnitude of diabetes-associated cognitive decrements in the present study are comparable with those reported in systematic reviews ${ }^{48}$ and show that type 2 diabetes is associated with mild decrements in multiple cognitive domains. In this context, the observed interaction between diabetes and hypertension suggests that individuals with both conditions are at especially high risk of cognitive impairment, particularly in the domain of executive function and attention. Our finding that type 2 diabetes was more strongly associated with aortic than with carotid stiffness is consistent with the concept that diabetes may affect the stiffness of muscular arteries more strongly, or earlier, than of elastic arteries. ${ }^{12,49}$ In addition, the overall good glycaemic control of individuals with diabetes in our cohort ( $\mathrm{HbA} 1 \mathrm{c} 6.9 \%$ [52 mmol/mol]) may have attenuated the association between diabetes and carotid stiffness in our cohort, because glycaemia and insulinaemia are major determinants of (carotid) arterial stiffness in diabetes. ${ }^{12}$, 50-52 Obviously, the weak associations observed between type 2 diabetes and carotid arterial stiffness made a mediation effect of carotid arterial stiffness on the relationship between type 2 diabetes and cognitive performance a priori unlikely. Therefore, we cannot exclude that such mediation effects exist in diabetic individuals with more severe carotid stiffening. In addition, our results do not preclude the possibility that other 
vascular factors, such as (carotid) atherosclerosis (ie, large vessel pathology) or cerebral small vessel disease (ie, small vessel pathology), rather than or in addition to metabolic variables, mediate the negative effects of type 2 diabetes on cognitive performance.

Strengths of this study include the evaluation of both aortic and local carotid arterial stiffness and the comprehensive set of demographic, haemodynamic and cardiovascular variables that could be taken into account in the analyses. Our study also had limitations. First, we used a complete case analysis approach, which may have resulted in underestimation of associations as individuals who were excluded were generally slightly less healthy (Table S3.1, Supplement). Notably, however, most of the missing data were unavailable due to logical or technical reasons. Data imputation was considered, but as The Maastricht Study is an ongoing study that aims to include 10,000 individuals, and the accuracy and validity of imputation is likely to increase with increasing sample size and increasing amount of data available, it was decided to wait until data collection is completed before missing values will be imputed. A second limitation is the fact that we were unable to capture all aspects of cognitive functioning, which may also have led us to underestimate the true association between arterial stiffness and cognitive performance. Third, we did not adjust for multiple testing, thereby increasing the possibility of false positive findings. We nonetheless believe that it is unlikely that the pattern we observed, and that was consistent across multiple sensitivity analyses, is solely explained by the play of chance. A fourth limitation concerns the accuracy of the calibration method used to determine local carotid PP. Specifically, we cannot exclude the possibility of a systematic error in our measurement of brachial MAP with resulting errors in the absolute values of carotid PP. Note, however, that such a systematic error would not affect the ranking of individuals, and hence is unlikely to have affected the outcome of the sensitivity analyses in which local carotid PP was used. Last, although we think that it is unlikely that (overall quite subtle) cognitive impairment leads to stiffening of the arteries, the cross-sectional nature of our study does not permit any conclusions on causality.

In conclusion, the present study shows that carotid arterial stiffness is associated with cognitive performance, but does not mediate the relationship between type 2 diabetes and cognitive dysfunction. Our findings indicate that stiffening of elastic and muscular arteries may differentially affect brain function, highlighting the importance of markers of carotid stiffness in studies linking arterial stiffening to brain function. Future (longitudinal) studies are warranted to confirm that our findings are not just a chance finding and may shed light on the mechanisms involved. Hypothetically, our results suggest that prevention and treatment of increased carotid stiffness could serve as new targets to preserve cognitive function, at least on a population level. The effectiveness of 
interventions targeting carotid stiffness on the individual level might be limited given the rather weak associations observed, which, however, does not preclude carotid stiffening from being an causative factor for cognitive deterioration and the development of dementia. 


\section{References}

1. Plassman, B.L., et al., Prevalence of cognitive impairment without dementia in the United States. Ann Intern Med, 2008. 148(6): p. 427-34.

2. Gorelick, P.B., et al., Vascular contributions to cognitive impairment and dementia: a statement for healthcare professionals from the american heart association/american stroke association. Stroke, 2011. 42(9): p. 2672-713.

3. Zieman, S.J., V. Melenovsky, and D.A. Kass, Mechanisms, pathophysiology, and therapy of arterial stiffness. Arterioscler Thromb Vasc Biol, 2005. 25(5): p. 932-43.

4. Mitchell, G.F., et al., Arterial stiffness, pressure and flow pulsatility and brain structure and function: the Age, Gene/Environment Susceptibility--Reykjavik study. Brain, 2011. 134(Pt 11): p. 3398-407.

5. O'Rourke, M.F. and M.E. Safar, Relationship between aortic stiffening and microvascular disease in brain and kidney: cause and logic of therapy. Hypertension, 2005. 46(1): p. 200-4.

6. Van Sloten, T.T., et al., Association between arterial stiffness, cerebral small vessel disease and cognitive impairment: A systematic review and meta-analysis. Neurosci Biobehav Rev, 2015. 53: p. 121-30.

7. Laurent, S., et al., Expert consensus document on arterial stiffness: methodological issues and clinical applications. Eur Heart J, 2006. 27(21): p. 2588-605.

8. Poels, M.M., et al., Arterial stiffness, cognitive decline, and risk of dementia: the Rotterdam study. Stroke, 2007. 38(3): p. 888-92.

9. Tarumi, T., et al., Central artery stiffness, neuropsychological function, and cerebral perfusion in sedentary and endurance-trained middle-aged adults. J Hypertens, 2013. 31(12): p. 24009.

10. Biessels, G.J., et al., Dementia and cognitive decline in type 2 diabetes and prediabetic stages: towards targeted interventions. Lancet Diabetes Endocrinol, 2014. 2(3): p. 246-55.

11. Stehouwer, C.D., R.M. Henry, and I. Ferreira, Arterial stiffness in diabetes and the metabolic syndrome: a pathway to cardiovascular disease. Diabetologia, 2008. 51(4): p. 527-39.

12. Henry, R.M., et al., Arterial stiffness increases with deteriorating glucose tolerance status: the Hoorn Study. Circulation, 2003. 107(16): p. 2089-95.

13. Kimoto, E., et al., Regional arterial stiffness in patients with type 2 diabetes and chronic kidney disease. J Am Soc Nephrol, 2006. 17(8): p. 2245-52.

14. Rema, M., et al., Association of carotid intima-media thickness and arterial stiffness with diabetic retinopathy: the Chennai Urban Rural Epidemiology Study (CURES-2). Diabetes Care, 2004. 27(8): p. 1962-7.

15. Schram, M.T., et al., The Maastricht Study: an extensive phenotyping study on determinants of type 2 diabetes, its complications and its comorbidities. Eur J Epidemiol, 2014. 29(6): p. 439-51.

16. World Health Organisation. Definition and diagnosis of diabetes mellitus and intermediate hyperglycemia: report of a WHO/IDF consultation. 2006. Available at: http://www.idf.org/ webdata/docs/WHO_IDF_definition_diagnosis_of_diabetes.pdf. Accessed July 13, 2015.

17. Van Bortel, L.M., et al., Expert consensus document on the measurement of aortic stiffness in daily practice using carotid-femoral pulse wave velocity. J Hypertens, 2012. 30(3): p. 445-8.

18. Hermeling, E., et al., The dicrotic notch as alternative time-reference point to measure local pulse wave velocity in the carotid artery by means of ultrasonography. J Hypertens, 2009. 27(10): p. 2028-35.

19. Willekes, C., et al., Evaluation of off-line automated intima-media thickness detection of the common carotid artery based on M-line signal processing. Ultrasound Med Biol, 1999. 25(1): p. 57-64.

20. Reneman, R.S., J.M. Meinders, and A.P. Hoeks, Non-invasive ultrasound in arterial wall dynamics in humans: what have we learned and what remains to be solved. Eur Heart J, 2005. 
26(10): p. 960-6.

21. Rothman, K.J., No adjustments are needed for multiple comparisons. Epidemiology, 1990. 1(1): p. 43-6.

22. Preacher, K.J. and A.F. Hayes, Asymptotic and resampling strategies for assessing and comparing indirect effects in multiple mediator models. Behav Res Methods, 2008. 40(3): p. 879-91.

23. Paini, A., et al., Carotid and aortic stiffness: determinants of discrepancies. Hypertension, 2006. 47(3): p. 371-6.

24. Maher, E., et al., Site specific inelasticity of arterial tissue. J Biomech, 2012. 45(8): p. 1393-9.

25. Van Sloten, T.T., et al., Local stiffness of the carotid and femoral artery is associated with incident cardiovascular events and all-cause mortality: the Hoorn study. J Am Coll Cardiol, 2014. 63(17): p. 1739-47.

26. Yang, E.Y., et al., Carotid arterial wall characteristics are associated with incident ischemic stroke but not coronary heart disease in the Atherosclerosis Risk in Communities (ARIC) study. Stroke, 2012. 43(1): p. 103-8.

27. Lim, S.L., et al., Vascular Health Indices and Cognitive Domain Function: Singapore Longitudinal Ageing Studies. J Alzheimers Dis, 2015. 50(1): p. 27-40.

28. Hanon, O., et al., Relationship between arterial stiffness and cognitive function in elderly subjects with complaints of memory loss. Stroke, 2005. 36(10): p. 2193-7.

29. Scuteri, A., et al., Arterial stiffness is an independent risk factor for cognitive impairment in the elderly: a pilot study. J Hypertens, 2005. 23(6): p. 1211-6.

30. Scuteri, A., et al., Aortic stiffness and hypotension episodes are associated with impaired cognitive function in older subjects with subjective complaints of memory loss. Int J Cardiol, 2013. 169(5): p. 371-7.

31. Singer, J., et al., The association between pulse wave velocity and cognitive function: the Sydney Memory and Ageing Study. PLoS One, 2013. 8(4): p. e61855.

32. Watson, N.L., et al., Arterial stiffness and cognitive decline in well-functioning older adults. J Gerontol A Biol Sci Med Sci, 2011. 66(12): p. 1336-42.

33. Zhong, W., et al., Pulse wave velocity and cognitive function in older adults. Alzheimer Dis Assoc Disord, 2014. 28(1): p. 44-9.

34. Pase, M.P., et al., Association of Aortic Stiffness With Cognition and Brain Aging in Young and Middle-Aged Adults: The Framingham Third Generation Cohort Study. Hypertension, 2016. 67(3): p. 513-9.

35. Hajjar, I., et al., Roles of Arterial Stiffness and Blood Pressure in Hypertension-Associated Cognitive Decline in Healthy Adults. Hypertension, 2016. 67(1): p. 171-5.

36. Muller, M., et al., Cardiovascular disease and cognitive performance in middle-aged and elderly men. Atherosclerosis, 2007. 190(1): p. 143-9.

37. Kearney-Schwartz, A., et al., Vascular structure and function is correlated to cognitive performance and white matter hyperintensities in older hypertensive patients with subjective memory complaints. Stroke, 2009. 40(4): p. 1229-36.

38. Tsao, C.W., et al., Association of arterial stiffness with progression of subclinical brain and cognitive disease. Neurology, 2016. 86(7): p. 619-26.

39. Benetos, A., et al., Pulse wave velocity is associated with 1-year cognitive decline in the elderly older than 80 years: the PARTAGE study. J Am Med Dir Assoc, 2012. 13(3): p. 239-43.

40. Waldstein, S.R., et al., Pulse pressure and pulse wave velocity are related to cognitive decline in the Baltimore Longitudinal Study of Aging. Hypertension, 2008. 51(1): p. 99-104.

41. Elias, M.F., et al., Arterial pulse wave velocity and cognition with advancing age. Hypertension, 2009. 53(4): p. 668-73.

42. Tsao, C.W., et al., Relations of arterial stiffness and endothelial function to brain aging in the community. Neurology, 2013. 81(11): p. 984-91.

43. Gustavsson, A.M., et al., Cerebral Microbleeds and White Matter Hyperintensities in 
Cognitively Healthy Elderly: A Cross-Sectional Cohort Study Evaluating the Effect of Arterial Stiffness. Cerebrovasc Dis Extra, 2015. 5(2): p. 41-51.

44. Laurent, S., M. Briet, and P. Boutouyrie, Large and small artery cross-talk and recent morbiditymortality trials in hypertension. Hypertension, 2009. 54(2): p. 388-92.

45. Brisset, M., et al., Large-vessel correlates of cerebral small-vessel disease. Neurology, 2013. 80(7): p. 662-9.

46. Jochemsen, H.M., et al., Arterial stiffness and progression of structural brain changes: The SMART-MR study. Neurology, 2015. 84(5): p. 448-55.

47. Mukai, S., et al., Effect of systolic blood pressure and carotid stiffness on baroreflex gain in elderly subjects. J Gerontol A Biol Sci Med Sci, 2003. 58(7): p. 626-30.

48. Van den Berg, E., et al., Type 2 diabetes mellitus, hypertension, dyslipidemia and obesity: A systematic comparison of their impact on cognition. Biochim Biophys Acta, 2009. 1792(5): p. 470-81.

49. Schram, M.T., et al., Increased central artery stiffness in impaired glucose metabolism and type 2 diabetes: the Hoorn Study. Hypertension, 2004. 43(2): p. 176-81.

50. Salomaa, V., et al., Non-insulin-dependent diabetes mellitus and fasting glucose and insulin concentrations are associated with arterial stiffness indexes. The ARIC Study. Atherosclerosis Risk in Communities Study. Circulation, 1995. 91(5): p. 1432-43.

51. Emoto, M., et al., Stiffness indexes beta of the common carotid and femoral arteries are associated with insulin resistance in NIDDM. Diabetes Care, 1998. 21(7): p. 1178-82.

52. Van Dijk, R.A., et al., Associations of metabolic variables with arterial stiffness in type 2 diabetes mellitus: focus on insulin sensitivity and postprandial triglyceridaemia. Eur J Clin Invest, 2003. 33(4): p. 307-15. 
Arterial stiffness, cognition, and type 2 diabetes 


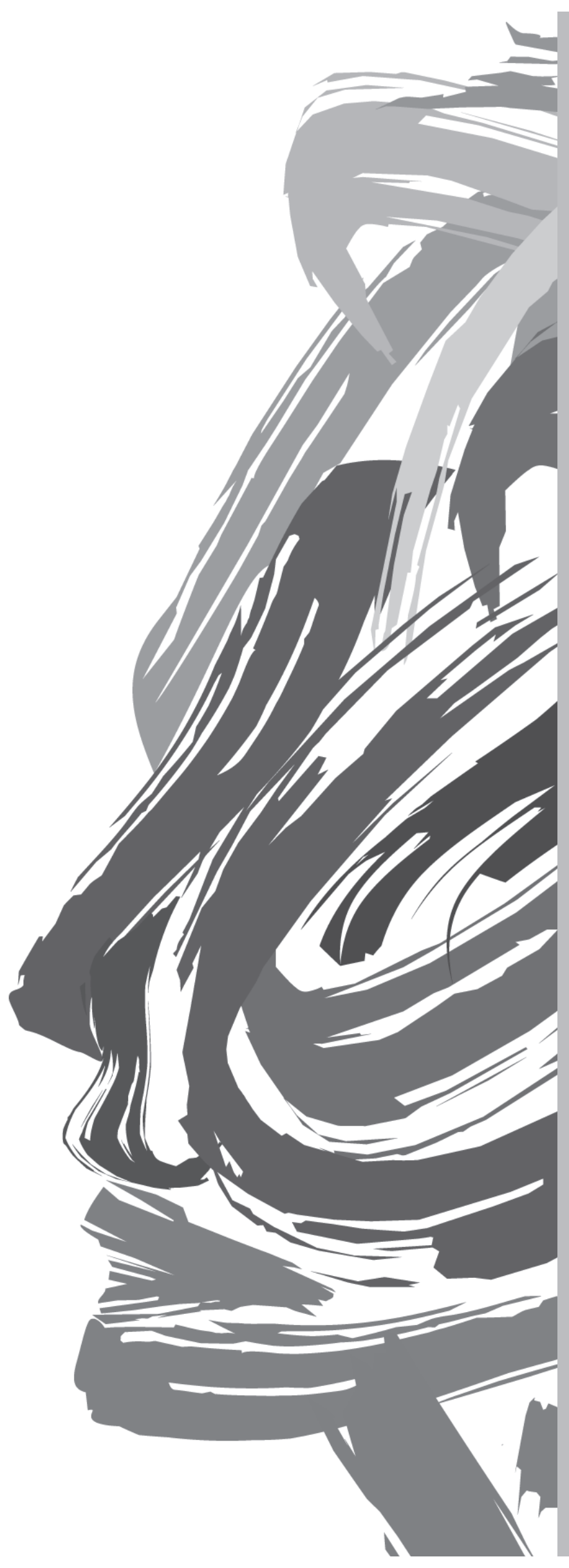


Supplement to

Chapter 3 


\section{Extended methods}

\section{Study population}

The Maastricht Study focuses on the aetiology, pathophysiology, complications, and comorbidities of type 2 diabetes and is characterised by an extensive phenotyping approach. ${ }^{1}$ All individuals aged between 40 and 75 years living in the southern part of the Netherlands, sufficiently proficient in the Dutch language were eligible for participation. Participants were recruited through mass media campaigns and from the municipal registries and the regional Diabetes Patient Registry via mailings. Recruitment was stratified according to known type 2 diabetes status for reasons of efficiency.

As part of the extensive phenotyping approach, all participants were subjected to a large number of measurements that were performed by trained research assistants during three four-hour visits to The Maastricht Study research centre using standardised protocols. Each participant completed all visits, and thus examinations, within a time window of three months. Prior to all visits, participants were asked to refrain from smoking and drinking coffee or tea or alcohol beverages for at least three hours. Participants were allowed to have a light meal (breakfast and/or lunch), except prior to the first visit, were participants were requested to visit the research centre in a fasted state. For the majority of the participants, both cognitive assessment and measurement of arterial stiffness took place during the second visit. Due to logistical reasons, some participants underwent vascular ultrasound during their third visit to the research centre.

\section{Cognitive assessment}

A concise battery (30 $\mathrm{min}$ ) of neuropsychological tests was administered to assess cognitive performance. ${ }^{1}$ For conceptual clarity, and to reduce the number of cognitive outcomes, test results were divided into three cognitive domains (ie, free recall memory, processing speed, and executive function and attention). The composite free recall memory score was derived from the Verbal Learning Test by weighting total immediate and delayed recall scores. The domain processing speed included the Stroop Colour Word Test Part I and II, the Concept Shifting Test Part A and B, and the Letter-Digit Substitution Test. Executive function and attention was assessed by the Stroop Colour Word Test Part III and the Concept Shifting Test Part C. A brief description of the individual tests is provided on the next page. Raw test scores were transformed into z-scores. Standardised scores of the Stroop Colour Word Test and Concept Shifting Test were inverted so that higher scores indicated better cognitive performance. Thereafter, domain-specific scores were calculated by averaging the z-scores from (sub)tests within that domain (eg, free recall memory $=z$-score ${ }_{\text {immediate recall }}+z$-score dealyed recall $/ 2$ ). 
Description of the individual cognitive tests used in the present study:

\section{Verbal Learning Test $^{2}$}

Fifteen unrelated, monosyllabic, words were presented on a computer screen in five subsequent trials. After each trial, participants were instructed to recall as many words as possible in any order. Twenty minutes after the last trial, participants were asked again to reproduce the words. Outcomes recorded included the total number of words correctly recalled over the five trials (total immediate recall) and the number of correctly recalled words during delayed recall (delayed recall).

\section{Stroop Colour Word Test ${ }^{3}$}

In this test, that consisted of three parts, participants were firstly asked to read aloud colour names (ie, red, blue, yellow, and green) that were printed in black ink (Part I). Secondly, they were instructed to name solid colour patches (Part II). Finally, participants had to name the ink colour of colour names that were printed in an incongruent colour (eg, participants were asked to say red when the word yellow was printed in red) (Part III). The time needed to complete Part III was adjusted for the average time needed to complete Part I and II.

\section{Concept Shifting Test ${ }^{4}$}

This test, a modification of the Trailing Making Test, consisted of four subtasks. During each subtask, participants were shown 16 small circles aligned along a larger imaginary circle. The small circles contained (a combination of) digits, letters, or were empty. Participants were instructed to cross-out as quickly as possible the digits in ascending order (Part A), the letters in alphabetic order (Part B), and the letters and digits in alternating order (Part C). Thereafter, participants were asked to cross-out empty circles in a clockwise fashion in two consecutive trials (Part 0). In this way, test results could be accounted for basic motor speed. The time needed to complete subtasks A and B was adjusted for the average time needed to complete Part 0 , the time needed to complete Part $\mathrm{C}$ for the average time of Part A and B.

\section{Letter-Digit Substitution Test ${ }^{5}$}

Participants were requested to match digits to letters according to a given key. This key included the numbers 1 to 9, each paired with a different letter. The outcome of interest was the number of correct substitutions within 90 seconds.

\section{Measures of arterial stiffness}

Arterial stiffness was assessed non-invasively by means of vascular ultrasound and applanation tonometry. All measurements (approximately $45 \mathrm{~min}$ ) were done by 
trained vascular technicians unaware of the participants' clinical or diabetes status. Measurements took place in a quiet temperature-controlled room $\left(21-23^{\circ} \mathrm{C}\right)$ and were performed in supine position, after $10 \mathrm{~min}$ of rest. Talking or sleeping was not allowed during the examination. A three-lead electrocardiogram was recorded continuously during the measurements to facilitate automatic signal processing. In addition, brachial systolic, diastolic, and mean arterial pressure (MAP) were determined repeatedly with a 5-min interval, using an oscillometric device (Accutorr Plus, Datascope Inc., Montvale, NJ, USA), and the average of these measurements was calculated.

\section{Aortic stiffness}

Carotid-to-femoral pulse wave velocity (cfPWV) was determined according to recent guidelines ${ }^{6}$ with the use of applanation tonometry (SphygmoCor, Atcor Medical, Sydney, Australia). Pressure waveforms were determined the right common carotid and right common femoral arteries. The difference in the time of pulse arrival from the R-wave of the electrocardiogram between the two sites (transit time) was determined with the intersecting tangents algorithm. The pulse wave travel distance was calculated as $80 \%$ of the direct straight distance (measured with an infantometer) between the two arterial sites. The median of three consecutive carotid-to-femoral pulse wave velocity (defined as travelled distance / transit time) recordings was used in the analyses.

\section{Carotid stiffness}

Elastic properties of the left common carotid artery (at least $10 \mathrm{~mm}$ proximal to the carotid bulb) were obtained by using an ultrasound scanner equipped with a 7.5$\mathrm{MHz}$ linear probe (MyLab 70, Esaote Europe B.V., Maastricht, the Netherlands). This setup enabled the measurement of diameter (D), distension (DS) and intima-media thickness (IMT) as described in more detail elsewhere. ${ }^{7,8}$ In brief, during the ultrasound measurements, a B-mode image on the basis of $19 \mathrm{M}$-lines was depicted on screen and an online echo-tracking algorithm showed real-time anterior and posterior arterial wall displacements. The M-mode recordings were composed of 19 simultaneous recordings at a frame rate of $498 \mathrm{~Hz}$. The distance between the $\mathrm{M}$-line recording positions was $0.96 \mathrm{~mm}$, thus, a total segment of $18.24 \mathrm{~mm}$ of each artery was covered by the scan plane. For offline processing, the radiofrequency signal was fed into a dedicated PCbased acquisition system (ART.LAB, Esaote Europe B.V. Maastricht, the Netherlands) with a sampling frequency of $50 \mathrm{MHz}$. Data processing was performed in MatLab (version 7.5, Mathworks, Natick, MA, USA). The distension waveforms were obtained from the radio frequency data with the use of a wall track algorithm. ${ }^{7}$ Carotid IMT was defined as the distance of the posterior wall from the leading edge interface between lumen and intima to the leading edge interface between media and adventitia. ${ }^{9}$ The median diameter, distension, and IMT of three measurements were used in the analyses. 
Combined with brachial pulse pressure (PP), these measures were used to calculate the following indices: ${ }^{10}$

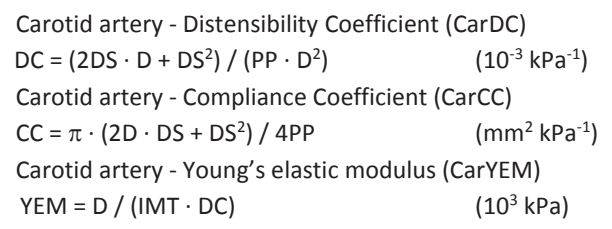

CarDC represents arterial stiffness, CarYEM the stiffness of the arterial wall material at operating pressure, and CarCC arterial buffering capacity. Please note that higher values of cfPWV and CarYEM, but lower values of CarDC and CarCC reflect greater arterial stiffness.

\section{Local carotid pulse pressure}

Pulse pressure at the carotid artery was calculated by calibrating the systolic-diastolic amplitude of the carotid artery tonometry waveform (sys-dias) tono $_{\text {o }}$ to pressure, assuming a constant difference between mean arterial pressure (MAP) and diastolic pressure (DP) along the large arteries: PPcar $=(\text { sys-dias })_{\text {tono }} *(\mathrm{MAP}-\mathrm{DP})_{\text {brach }} /(\text { mean-dias })_{\text {tono }}$. This procedure is the same as those described by Kelly and Fitchett ${ }^{11}$ and Van Bortel et al., ${ }^{12}$ except that the MAP and DP values at the brachial artery were taken as the respective averages over the vascular measurements (ie, over a 30 to 45 min period) as obtained with a validated commercial oscillometric device (Accutorr Plus, Datascope Inc., Montvale, NJ, USA). Cited procedures considered the mean-diastolic difference as obtained from a measured brachial artery pressure or diameter waveform.

The calibration approach used in the present study was chosen because of its practical applicability in a large-scale population-based study. We acknowledge that there are other methods to determine local carotid pulse pressure non-invasively, which include, for example, rescaling carotid distension waveforms with use of brachial distension waveforms, rescaling carotid tonometry waveforms with use of brachial mean arterial pressure obtained from brachial tonometry, and calculating carotid pressure from radial tonometry with use of a transfer function. Given the lively debate regarding the reliability and validity of the different methods available, ${ }^{13-15}$ we believe that a consensus on the best method remains elusive.

\section{Reproducibility}

Reproducibility was assessed in 12 individuals ( 6 men; $60.8 \pm 6.8$ years; 6 with type 2 diabetes) who were examined by two observers at two occasions spaced one week apart. The intra- and inter-observer intra-class correlation coefficients were 0.87 and 0.69 
for carotid-to-femoral pulse wave velocity; 0.85 and 0.73 for the carotid distensibility coefficient, and 0.95 and 0.72 for the carotid compliance coefficient.

\section{Covariates}

Web-based questionnaires were used to obtain information regarding smoking behaviour (never/former/current), alcohol consumption, and prior cardiovascular disease, as described in more detail elsewhere. ${ }^{1}$ Alcohol consumption was classified as none, low (1-7 glasses per week for women, 1-14 glasses for men), or high (>7 glasses per week for women, $>14$ for men). Prior cardiovascular disease was defined as a history of myocardial infarction, stroke, or vascular surgery (including angioplasty) of coronary, carotid, abdominal aortic, or peripheral arteries. Serum concentrations of total cholesterol, highdensity lipoprotein (HDL) cholesterol, triglycerides, and creatinine were measured using an automatic analyser (Beckman Synchron LX20, Beckman Coulter Inc., Brea, USA). A particle enhanced immunoturbidimetric assay (Roche Cobas 8000, Roche Diagnostics, Basel, Switzerland) was used to measure cystatin C, which was combined with serum creatinine to estimate the glomerular filtration rate using the Chronic Kidney Disease Epidemiology Collaboration (CKD-EPI) equation. ${ }^{16}$ Office blood pressure was calculated as the average of at least three blood pressure readings (Omron 705IT, Japan) performed after a minimum of 10 min rest. Body Mass Index was calculated as weight $(\mathrm{kg})$ divided by height squared $\left(\mathrm{m}^{2}\right)$, medication use was determined as described previously. ${ }^{1}$ Educational level was divided into three categories: [1] low (ie, no education, primary education, or lower vocational education); [2] intermediate (ie, intermediate general secondary education, intermediate vocational education, or higher general secondary education), and [3] high (ie, higher vocational education or university). The presence of a current depression was evaluated using the Mini International Neuropsychiatric Interview (MINI). ${ }^{17}$

\section{Extended results}

\section{Sensitivity analyses}

Data on 24-hour blood pressure monitoring were available for 647 individuals, of whom 634 also had data on indices of carotid stiffness. Adjustment for 24-hour mean arterial pressure (and heart rate) instead of the average mean arterial pressure (and heart rate) during vascular assessment did not substantially change the associations between type 2 diabetes and arterial stiffness or between arterial stiffness and cognitive performance (data not shown). The same was true for the association between type 2 diabetes and cognitive dysfunction when alternatively adjusted for 24-hour systolic and diastolic blood pressure, or for mean arterial pressure during vascular assessment, instead of office-based systolic and diastolic blood pressure (data not shown). 
Data on physical activity was available for 625 individuals ( $n=612$ with data on carotid stiffness). Additional adjustments for physical activity did not affect the main results (data not shown). 


\section{References}

1. Schram, M.T., et al., The Maastricht Study: an extensive phenotyping study on determinants of type 2 diabetes, its complications and its comorbidities. Eur J Epidemiol, 2014. 29(6): p. 439-51.

2. Van der Elst, W., et al., Rey's verbal learning test: normative data for 1855 healthy participants aged 24-81 years and the influence of age, sex, education, and mode of presentation. J Int Neuropsychol Soc, 2005. 11(3): p. 290-302.

3. Van der Elst, W., et al., The Stroop color-word test: influence of age, sex, and education; and normative data for a large sample across the adult age range. Assessment, 2006. 13(1): p. 6279.

4. Van der Elst, W., et al., The Concept Shifting Test: adult normative data. Psychol Assess, 2006. 18(4): p. 424-32.

5. Van der Elst, W., et al., The Letter Digit Substitution Test: normative data for 1,858 healthy participants aged 24-81 from the Maastricht Aging Study (MAAS): influence of age, education, and sex. J Clin Exp Neuropsychol, 2006. 28(6): p. 998-1009.

6. Van Bortel, L.M., et al., Expert consensus document on the measurement of aortic stiffness in daily practice using carotid-femoral pulse wave velocity. J Hypertens, 2012. 30(3): p. 445-8.

7. Hermeling, E., et al., The dicrotic notch as alternative time-reference point to measure local pulse wave velocity in the carotid artery by means of ultrasonography. J Hypertens, 2009. 27(10): p. 2028-35.

8. Willekes, C., et al., Evaluation of off-line automated intima-media thickness detection of the common carotid artery based on M-line signal processing. Ultrasound Med Biol, 1999. 25(1): p. 57-64.

9. Willekes, C., et al., Evaluation of off-line automated intima-media thickness detection of the common carotid artery based on M-line signal processing. Ultrasound in medicine \& biology, 1999. 25(1): p. 57-64.

10. Reneman, R.S., J.M. Meinders, and A.P. Hoeks, Non-invasive ultrasound in arterial wall dynamics in humans: what have we learned and what remains to be solved. Eur Heart J, 2005. 26(10): p. 960-6.

11. Kelly, R. and D. Fitchett, Noninvasive determination of aortic input impedance and external left ventricular power output: a validation and repeatability study of a new technique. J Am Coll Cardiol, 1992. 20(4): p. 952-63.

12. Van Bortel, L.M., et al., Non-invasive assessment of local arterial pulse pressure: comparison of applanation tonometry and echo-tracking. J Hypertens, 2001. 19(6): p. 1037-44.

13. Verbeke, F., et al., Noninvasive assessment of local pulse pressure: importance of brachial-toradial pressure amplification. Hypertension, 2005. 46(1): p. 244-8.

14. Adji, A. and M.F. O'Rourke, Brachial artery tonometry and the Popeye phenomenon: explanation of anomalies in generating central from upper limb pressure waveforms. J Hypertens, 2012. 30(8): p. 1540-51.

15. Lehmann, E.D., Regarding the accuracy of generalized transfer functions for estimating central aortic blood pressure when calibrated non-invasively. J Hypertens, 2000. 18(3): p. 347-49; author reply 349-50.

16. Inker, L.A., et al., Estimating glomerular filtration rate from serum creatinine and cystatin C. N Engl J Med, 2012. 367(1): p. 20-9.

17. Sheehan, D.V., et al., The Mini-International Neuropsychiatric Interview (M.I.N.I.): the development and validation of a structured diagnostic psychiatric interview for DSM-IV and ICD-10. J Clin Psychiatry, 1998. 59 Suppl 20: p. 22-33;quiz 34-57. 
Table S3.1 Characteristics of individuals with complete and incomplete data in the present study

\begin{tabular}{|c|c|c|c|c|}
\hline Clinical characteristics & $\begin{array}{c}\text { Included } \\
(\mathrm{n}=725)\end{array}$ & $\begin{array}{c}\text { Excluded } \\
(n=134)\end{array}$ & Missing* & p-value ${ }^{\dagger}$ \\
\hline Age (years) & $60 \pm 8$ & $61 \pm 9$ & 0 & 0.041 \\
\hline Male & $396(54.6 \%)$ & $74(55.2 \%)$ & 0 & 0.897 \\
\hline Type 2 diabetes & $197(27.2 \%)$ & $54(40.3 \%)$ & 0 & 0.002 \\
\hline Educational level & $113 / 302 / 310$ & $29 / 52 / 53$ & 0 & 0.222 \\
\hline (low/middle/high) & $(15.6 \% / 41.7 \% / 42.8 \%)$ & $(21.6 \% / 38.8 \% / 39.6 \%)$ & & \\
\hline BMI $\left(\mathrm{kg} / \mathrm{m}^{2}\right)$ & $27.2 \pm 4.4$ & $28.0 \pm 5.1$ & 1 & 0.080 \\
\hline Total cholesterol (mmol/L) & $5.2 \pm 1.2$ & $5.0 \pm 1.2$ & 7 & 0.052 \\
\hline HDL-cholesterol (mmol/L) & $1.3 \pm 0.4$ & $1.3 \pm 0.5$ & 7 & 0.223 \\
\hline Total/HDL cholesterol ratio & $4.2 \pm 1.2$ & $4.3 \pm 1.3$ & 8 & 0.700 \\
\hline Triglycerides (mmol/L) & $1.2[0.9-1.8]$ & 1.4 [0.9-1.9] & 7 & 0.099 \\
\hline Lipid lowering medication & $255(35.2 \%)$ & $61(45.5 \%)$ & 0 & 0.015 \\
\hline Systolic blood pressure $(\mathrm{mmHg})^{\ddagger}$ & $137 \pm 19$ & $138 \pm 20$ & 2 & 0.861 \\
\hline Diastolic blood pressure $(\mathrm{mmHg})^{\ddagger}$ & $77 \pm 11$ & $77 \pm 9$ & 2 & 0.869 \\
\hline Mean arterial pressure $(\mathrm{mmHg})^{\S}$ & $97 \pm 10$ & $100 \pm 12$ & 17 & 0.009 \\
\hline Heart rate $(\mathrm{bpm})^{\S}$ & $63 \pm 9$ & $65 \pm 8$ & 18 & 0.089 \\
\hline Hypertension" & $413(57.0 \%)$ & $85(64.4 \%)$ & 2 & 0.067 \\
\hline Antihypertensive medication & $283(39.0 \%)$ & $61(45.5 \%)$ & 0 & 0.159 \\
\hline eGFR $\left(\mathrm{ml} / \mathrm{min} / 1.73 \mathrm{~m}^{2}\right)$ & $89 \pm 15$ & $83 \pm 18$ & & 0.002 \\
\hline $\begin{array}{l}\text { Carotid-to-femoral pulse wave } \\
\text { velocity }(\mathrm{m} / \mathrm{s})\end{array}$ & $8.9 \pm 2.1$ & $9.8 \pm 2.6$ & 41 & 0.002 \\
\hline $\begin{array}{l}\text { Carotid artery - distensibility } \\
\text { coefficient }\left(10^{-3} / \mathrm{kPa}\right)^{\#}\end{array}$ & $13.7 \pm 4.8$ & $13.2 \pm 6.1$ & 26 & 0.442 \\
\hline $\begin{array}{l}\text { Carotid artery - compliance } \\
\text { coefficient }\left(\mathrm{mm}^{2} / \mathrm{kPa}\right)^{\#}\end{array}$ & $0.65 \pm 0.26$ & $0.65 \pm 0.41$ & 26 & 0.886 \\
\hline $\begin{array}{l}\text { Carotid artery - Young's elastic } \\
\text { modulus }\left(10^{3} / \mathrm{kPa}\right)^{\#}\end{array}$ & $0.78 \pm 0.36$ & $0.86 \pm 0.41$ & 26 & 0.054 \\
\hline Alcohol consumption & $120 / 381 / 224$ & $29 / 59 / 27$ & 19 & 0.048 \\
\hline (no/low/high) & $(16.6 \% / 52.6 \% / 30.9 \%)$ & $(25.2 \% / 51.3 \% / 23.5 \%)$ & & \\
\hline Smoking behaviour & $226 / 384 / 115$ & $33 / 62 / 23$ & 16 & 0.560 \\
\hline (never/former/current) & $(31.2 \% / 53.0 \% / 15.9 \%)$ & $(28.0 \% / 52.5 \% / 19.5 \%)$ & & \\
\hline Prior cardiovascular disease & $125(17.2 \%)$ & $25(25.5 \%)$ & 36 & 0.047 \\
\hline Current depression & $28(3.9 \%)$ & $11(8.7 \%)$ & 7 & 0.017 \\
\hline Free recall memory & $0.05 \pm 0.95$ & $-0.16 \pm 0.99$ & 9 & 0.030 \\
\hline Processing speed & $0.04 \pm 0.79$ & $-0.11 \pm 0.78$ & 14 & 0.065 \\
\hline Executive function \& attention & $0.03 \pm 0.81$ & $-0.10 \pm 0.89$ & 17 & 0.109 \\
\hline
\end{tabular}

Data are presented as mean \pm SD, median [IQR], or $n(\%) .{ }^{*}$ Number of missings for a specific variable. ${ }^{+} \mathrm{P}$-value for difference between individuals with and without complete data as evaluated with use of independent samples t-tests for continuous variables and Chi-square tests for categorical variables. ${ }^{\ddagger}$ Obtained from office blood pressure measurements. ${ }^{\S}$ Obtained from blood pressure measurements every 5 minutes during vascular assessment. "Hypertension was defined as a systolic blood pressure $\geq 140 \mathrm{mmHg}$ (based on at least three office blood pressure measurements), a diastolic blood pressure $\geq 90 \mathrm{mmHg}$, and/or current use of antihypertensive medication; "Data available from 711 out of the 725 individuals included in the present analyses. Abbreviations: $\mathrm{BMI}=$ Body Mass Index; HDL = high density lipoprotein; eGFR = estimated glomerular filtration rate. 
Table S3.2 Raw cognitive test results for participants with and without type 2 diabetes

\begin{tabular}{lccc}
\hline & $\begin{array}{c}\text { Type 2 diabetes } \\
\text { (n=197) }\end{array}$ & $\begin{array}{c}\text { No type 2 diabetes } \\
\text { (n=528) }\end{array}$ & p-value $^{*}$ \\
\hline Verbal Learning Test & & & \\
Total immediate recall (words) & $41 \pm 9$ & $47 \pm 9$ & $<0.001$ \\
Delayed recall (words) & $8 \pm 3$ & $10 \pm 3$ & $<0.001$ \\
Stroop Colour Word Test & $47[42-53]$ & $44[40-49]$ & $<0.001$ \\
Part I (seconds) & $62[56-71]$ & $56[51-63]$ & $<0.001$ \\
Part II (seconds) & $51[39-68]$ & $39[31-50]$ & $<0.001$ \\
Part II (seconds) & $16[13-20]$ & $14[12-18]$ & $<0.001$ \\
Concept Shifting Test & $21[17-25]$ & $18[15-22]$ & $<0.001$ \\
Part A (seconds) & $22[17-28]$ & $18[15-24]$ & $<0.001$ \\
Part B (seconds) & & $51 \pm 9$ & $<0.001$ \\
Part C (seconds) & $45 \pm 9$ & & \\
Letter Digit Substitution Test & & 510.001 \\
Correct substitutions (number) & & & \\
& & & \\
\hline
\end{tabular}

Data are presented as mean \pm SD or median [IQR]. ${ }^{*}$ P-value for difference between individuals with and without type 2 diabetes, derived from independent samples t-tests. A detailed description of the individual cognitive tests is found elsewhere in this Supplement.

Table S3.3 Mean difference in indices of carotid stiffness (calculated with local carotid artery pulse pressure) between individuals with and without type 2 diabetes

\begin{tabular}{|c|c|c|c|}
\hline & $\begin{array}{c}\text { Carotid artery - } \\
\text { distensibility coefficient } \\
\left(10^{-3} / \mathrm{kPa}\right)\end{array}$ & $\begin{array}{l}\text { Carotid artery - } \\
\text { compliance coefficient } \\
\left(\mathrm{mm}^{2} / \mathrm{kPa}\right)\end{array}$ & $\begin{array}{c}\text { Carotid artery - } \\
\text { Young's elastic modulus } \\
\left(10^{3} / \mathrm{kPa}\right)\end{array}$ \\
\hline \multicolumn{4}{|l|}{ Model } \\
\hline \multirow[t]{2}{*}{ Model 1} & $-1.041^{*}$ & -0.031 & 0.052 \\
\hline & $(-1.936$ to -0.145$)$ & (-0.076 to 0.014$)$ & (-0.009 to 0.114$)$ \\
\hline \multirow[t]{2}{*}{ Model 2} & $-0.936^{*}$ & -0.027 & 0.047 \\
\hline & $(-1.710$ to -0.162$)$ & (-0.069 to 0.015$)$ & (-0.010 to 0.105$)$ \\
\hline \multirow{2}{*}{ Model 3} & -0.390 & -0.039 & 0.025 \\
\hline & $(-1.309$ to 0.528$)$ & $(-0.089$ to 0.010$)$ & (-0.043 to 0.093$)$ \\
\hline
\end{tabular}

Data are presented as unstandardised regression coefficient $(95 \% \mathrm{Cl}) . \mathrm{N}=707 .{ }^{*} \mathrm{p}<0.05$. Individuals without diabetes were used as the reference group. Regression coefficients indicate the mean difference in indices of carotid stiffness between individuals with and without type 2 diabetes. Model 1: adjusted for sex and age; Model 2: additional adjustment for mean arterial pressure; Model 3: additional adjustments for body mass index, total/high density lipoprotein-cholesterol ratio, triglycerides, use of lipid-modifying medication, use of antihypertensive medication, estimated glomerular filtration rate, smoking behaviour, history of cardiovascular disease(s). Abbreviations: $\mathrm{Cl}=$ confidence interval. 
Table S3.4 Association between indices of carotid stiffness (calculated with local carotid artery pulse pressure) and cognitive performance

\begin{tabular}{|c|c|c|c|}
\hline Model & $\begin{array}{c}\text { Free recall } \\
\text { memory }\end{array}$ & Processing speed & $\begin{array}{c}\text { Executive function } \& \\
\text { attention }\end{array}$ \\
\hline \multicolumn{4}{|c|}{ Carotid artery - distensibility coefficient } \\
\hline \multirow[t]{2}{*}{ Model 1} & -0.043 & -0.059 & $-0.111^{*}$ \\
\hline & $(-0.118$ to 0.032$)$ & $(-0.134$ to 0.016$)$ & $(-0.188$ to -0.034$)$ \\
\hline \multirow[t]{2}{*}{ Model 2} & -0.035 & $-0.100^{*}$ & $-0.137^{*}$ \\
\hline & $(-0.121$ to 0.052$)$ & $(-0.186$ to -0.013$)$ & $(-0.227$ to -0.048$)$ \\
\hline \multirow[t]{2}{*}{ Model 3} & -0.019 & $-0.093^{*}$ & $-0.122^{*}$ \\
\hline & $(-0.105$ to 0.066$)$ & $(-0.180$ to -0.006$)$ & $(-0.212$ to -0.032$)$ \\
\hline \multicolumn{4}{|c|}{ Carotid artery - compliance coefficient } \\
\hline \multirow[t]{2}{*}{ Model 1} & -0.009 & -0.063 & $-0.082^{*}$ \\
\hline & $(-0.079$ to 0.061$)$ & $(-0.133$ to 0.007$)$ & $(-0.155$ to -0.009$)$ \\
\hline \multirow[t]{2}{*}{ Model 2} & 0.003 & $-0.085^{*}$ & $-0.088^{*}$ \\
\hline & $(-0.072$ to 0.078$)$ & $(-0.161$ to -0.010$)$ & $(-0.167$ to -0.010$)$ \\
\hline \multirow[t]{2}{*}{ Model 3} & -0.000 & $-0.087^{*}$ & $-0.082^{*}$ \\
\hline & $(-0.075$ to 0.074$)$ & $(-0.162$ to -0.011$)$ & $(-0.161$ to -0.004$)$ \\
\hline \multicolumn{4}{|c|}{ Carotid artery - Young's elastic modulus } \\
\hline \multirow[t]{2}{*}{ Model 1} & 0.003 & -0.044 & $-0.080^{*}$ \\
\hline & $(-0.065$ to 0.070$)$ & $(-0.111$ to 0.024$)$ & $(-0.150$ to -0.010$)$ \\
\hline \multirow[t]{2}{*}{ Model 2} & 0.016 & -0.062 & $-0.086^{*}$ \\
\hline & (-0.057 to 0.088$)$ & $(-0.135$ to 0.010$)$ & $(-0.161$ to -0.011$)$ \\
\hline \multirow[t]{2}{*}{ Model 3} & 0.024 & -0.058 & -0.073 \\
\hline & $(-0.047$ to 0.095$)$ & $(-0.130$ to 0.014$)$ & $(-0.147$ to 0.002$)$ \\
\hline
\end{tabular}

Data are presented as standardised regression coefficient $(95 \% \mathrm{Cl}) . \mathrm{N}=707 .{ }^{*} \mathrm{p}<0.05$. Regression coefficients indicate the change in cognitive performance per SD increase in carotid Young's elastic modulus and per SD decrease in carotid distensibility or compliance; in other words, a negative regression coefficient means that greater carotid stiffness is associated with worse cognitive performance. Model 1: adjusted for age, sex, and educational level; Model 2: additional adjustments for mean arterial pressure; Model 3: additional adjustments for presence of type 2 diabetes, body mass index, total/high density lipoprotein-cholesterol ratio, triglycerides, use of lipid-modifying medication, use of antihypertensive medication, estimated glomerular filtration rate, smoking behaviour, alcohol consumption, current depression, history of cardiovascular disease(s). Abbreviations: $\mathrm{Cl}=$ confidence interval; $\mathrm{SD}=$ standard deviation. 
Table S3.5 Association between the individual components of indices of carotid stiffness and cognitive performance

\begin{tabular}{|c|c|c|c|}
\hline Model & Free recall memory & Processing speed & $\begin{array}{c}\text { Executive function \& } \\
\text { attention }\end{array}$ \\
\hline \multicolumn{4}{|c|}{ Brachial pulse pressure $(n=711)$} \\
\hline \multirow[t]{2}{*}{ Model 1} & -0.029 & -0.009 & -0.049 \\
\hline & $(-0.096$ to 0.038$)$ & $(-0.077$ to 0.058$)$ & $(-0.118$ to 0.021$)$ \\
\hline \multirow[t]{2}{*}{ Model 2} & -0.016 & -0.048 & -0.069 \\
\hline & $(-0.104$ to 0.072$)$ & $(-0.137$ to 0.040$)$ & (-0.161 to 0.023$)$ \\
\hline \multirow[t]{2}{*}{ Model 3} & 0.033 & -0.007 & -0.026 \\
\hline & $(-0.056$ to 0.122$)$ & $(-0.098$ to 0.083$)$ & $(-0.120$ to 0.067$)$ \\
\hline \multicolumn{4}{|c|}{ Carotid pulse pressure $(n=708)$} \\
\hline \multirow[t]{2}{*}{ Model 1} & -0.021 & 0.012 & -0.029 \\
\hline & $(-0.090$ to 0.048$)$ & $(-0.057$ to 0.081$)$ & $(-0.101$ to 0.042$)$ \\
\hline \multirow[t]{2}{*}{ Model 2} & -0.002 & -0.012 & -0.034 \\
\hline & $(-0.092$ to 0.089$)$ & $(-0.103$ to 0.079$)$ & $(-0.128$ to 0.060$)$ \\
\hline \multirow[t]{2}{*}{ Model 3} & 0.035 & 0.019 & 0.005 \\
\hline & $(-0.056$ to 0.126$)$ & $(-0.073$ to 0.112$)$ & $(-0.091$ to 0.100$)$ \\
\hline \multicolumn{4}{|c|}{ Carotid diameter $(n=711)$} \\
\hline \multirow[t]{2}{*}{ Model 1} & -0.061 & 0.014 & -0.017 \\
\hline & $(-0.134$ to 0.012$)$ & $(-0.059$ to 0.088$)$ & $(-0.093$ to 0.059$)$ \\
\hline \multirow[t]{2}{*}{ Model 2} & -0.058 & 0.010 & -0.015 \\
\hline & $(-0.132$ to 0.017$)$ & $(-0.065$ to 0.084$)$ & $(-0.092$ to 0.062$)$ \\
\hline \multirow[t]{2}{*}{ Model 3} & -0.030 & 0.027 & 0.001 \\
\hline & $(-0.105$ to 0.045$)$ & $(-0.049$ to 0.104$)$ & $(-0.079$ to 0.080$)$ \\
\hline \multicolumn{4}{|c|}{ Carotid distension $(n=707)$} \\
\hline \multirow[t]{2}{*}{ Model 1} & 0.002 & 0.046 & $0.073^{*}$ \\
\hline & $(-0.064$ to 0.068$)$ & $(-0.020$ to 0.112$)$ & (0.005 to 0.142 ) \\
\hline \multirow[t]{2}{*}{ Model 2} & 0.003 & 0.046 & $0.074^{*}$ \\
\hline & $(-0.063$ to 0.069$)$ & $(-0.021$ to 0.112$)$ & (0.005 to 0.142 ) \\
\hline \multirow[t]{2}{*}{ Model 3} & 0.018 & 0.060 & $0.086^{*}$ \\
\hline & $(-0.047$ to 0.083$)$ & $(-0.006$ to 0.126$)$ & (0.017 to 0.154$)$ \\
\hline
\end{tabular}

Data are presented as standardised regression coefficient $(95 \% \mathrm{Cl}) . \mathrm{N}=707 .{ }^{*} \mathrm{p}<0.05$. Regression coefficients indicate the change in cognitive performance per SD increase in carotid Young's elastic modulus and per SD decrease in carotid distensibility or compliance; in other words, a negative regression coefficient means that greater carotid stiffness is associated with worse cognitive performance. Model 1: adjusted for age, sex, and educational level; Model 2: additional adjustments for mean arterial pressure; Model 3: additional adjustments for presence of type 2 diabetes, body mass index, total/high density lipoprotein-cholesterol ratio, triglycerides, use of lipid-modifying medication, use of antihypertensive medication, estimated glomerular filtration rate, smoking behaviour, alcohol consumption, current depression, history of cardiovascular disease(s). Abbreviations: $\mathrm{Cl}$ = confidence interval; $\mathrm{SD}=$ standard deviation. 
Table S3.6 Association of central pulse pressure and the augmentation index with cognitive performance

\begin{tabular}{|c|c|c|c|}
\hline Model & Free recall memory & Processing speed & $\begin{array}{c}\text { Executive function \& } \\
\text { attention }\end{array}$ \\
\hline \multicolumn{4}{|c|}{ Central pulse pressure $(n=711)$} \\
\hline \multirow[t]{2}{*}{ Model 1} & 0.003 & -0.001 & -0.000 \\
\hline & $(-0.065$ to 0.071$)$ & (-0.069 to 0.067$)$ & $(-0.071$ to 0.071$)$ \\
\hline \multirow[t]{2}{*}{ Model 2} & 0.017 & -0.023 & 0.005 \\
\hline & (-0.059 to 0.093$)$ & $(-0.100$ to 0.053$)$ & $(-0.074$ to 0.084$)$ \\
\hline \multirow[t]{2}{*}{ Model 3} & 0.034 & -0.002 & 0.028 \\
\hline & $(-0.042$ to 0.110$)$ & $(-0.078$ to 0.075$)$ & (-0.051 to 0.108$)$ \\
\hline \multicolumn{4}{|c|}{ Augmentation index $(n=711)$} \\
\hline \multirow[t]{2}{*}{ Model 1} & -0.011 & 0.028 & 0.049 \\
\hline & (-0.087 to 0.065$)$ & (-0.049 to 0.104$)$ & $(-0.030$ to 0.129$)$ \\
\hline \multirow[t]{2}{*}{ Model 2} & -0.033 & 0.008 & 0.053 \\
\hline & (-0.121 to 0.054$)$ & (-0.080 to 0.096$)$ & $(-0.038$ to 0.144$)$ \\
\hline \multirow[t]{2}{*}{ Model 3} & -0.040 & 0.017 & 0.067 \\
\hline & $(-0.128$ to 0.048$)$ & $(-0.073$ to 0.106$)$ & $(-0.026$ to 0.159$)$ \\
\hline
\end{tabular}

Data are presented as standardized regression coefficient $(95 \% \mathrm{Cl})$. Model 1: adjusted for age, sex, and educational level; Model 2: additional adjustments for mean arterial pressure and heart rate (the latter only for analyses of the augmentation index); Model 3: additional adjustments for presence of type 2 diabetes, body mass index, total/high density lipoprotein-cholesterol ratio, triglycerides, use of lipid-modifying medication, use of antihypertensive medication, estimated glomerular filtration rate, smoking behaviour, alcohol consumption, current depression, history of cardiovascular disease(s). Abbreviations: $\mathrm{Cl}=$ confidence interval; $\mathrm{SD}=$ standard deviation.

Table S3.7 Correlation between indices of carotid stiffness and carotid-to-femoral pulse wave velocity

\begin{tabular}{|c|c|c|c|c|}
\hline & cfPWV & CarDC ${ }^{*}$ & CarCC* & CarYEM \\
\hline \multirow[t]{2}{*}{ cfPWV } & 1 & 0.365 & 0.147 & 0.276 \\
\hline & $\ldots$ & $(<0.001)$ & $(<0.001)$ & $(<0.001)$ \\
\hline \multirow[t]{2}{*}{ CarDC $^{*}$} & & 1 & 0.797 & 0.746 \\
\hline & & $\ldots$ & $(<0.001)$ & $(<0.001)$ \\
\hline \multirow[t]{2}{*}{ CarCC $^{*}$} & & & 1 & 0.589 \\
\hline & & & $\ldots$ & $(<0.001)$ \\
\hline \multirow[t]{2}{*}{ CarYEM } & & & & 1 \\
\hline & & & & $\ldots$ \\
\hline
\end{tabular}

$\mathrm{N}=711$. Data are presented as Pearson correlation coefficient ( $\mathrm{p}$-value). ${ }^{*} \mathrm{CarDC}$ and CarCC were inverted so that higher values reflect greater arterial stiffness; Abbreviations: cfPWV = carotid-to-femoral pulse wave velocity; CarDC = carotid artery - distensibility coefficient; CarCC = carotid artery - compliance coefficient; CarYEM = carotid artery - Young's elastic modulus. 


\section{Chapter 4}

\section{CAROTID CIRCUMFERENTIAL WALL STRESS IS NOT ASSOCIATED WITH COGNITIVE PERFORMANCE AMONG INDIVIDUALS IN LATE MIDDLE AGE: THE MAASTRICHT STUDY}

Stefan L.C. Geijselaers, Simone J.S. Sep, Miranda T. Schram, Martin P.J. van Boxtel, Thomas T. van Sloten, Ronald M.A. Henry,

Koen D. Reesink, Nicolaas C. Schaper, Pieter C. Dagnelie, Carla J.H. van der Kallen, Geert Jan Biessels, Coen D.A. Stehouwer 


\section{Chapter 4}

\section{Abstract}

\section{Objective}

Arterial remodelling aims at normalising circumferential wall stress (CWS). Greater CWS in the carotid artery has previously been associated with the prevalence and severity of cerebral small vessel disease, a major cause of ageing-related cognitive decline. Here we test the hypothesis that greater carotid CWS is associated with poorer cognitive performance.

\section{Methods}

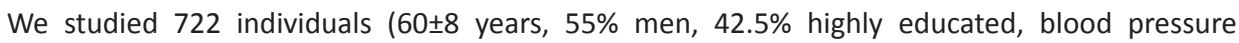
$137 \pm 19 / 77 \pm 11 \mathrm{mmHg}, \mathrm{n}=197$ with type 2 diabetes) who completed a neuropsychological assessment and underwent vascular ultrasound to measure the intima-media thickness (IMT) and interadventitial diameter (IAD) of the left common carotid artery at a plaque-free site. From IMT and IAD, lumen diameter (LD) was calculated. These structural measures were then combined with local carotid pulse pressure and brachial mean arterial pressure to obtain a measure of pulsatile (CWS pulsatile ) and average $\left(\mathrm{CWS}_{\text {mean }}\right.$ ) mechanical load on the vessel wall. Cognitive domains assessed were memory, executive function and attention, and processing speed.

\section{Results}

After adjustment for age, sex, and education, regression analyses showed that neither CWS nor $\mathrm{CWS}_{\text {mean }}$ were associated with measures of cognitive performance ( $p$-values $\geq 0.31$ ). This null association did not differ by age or sex, and was similar in individuals with and without diabetes. In addition, none of the individual measures of carotid structure (ie, IMT, IAD, and LD) was related to cognitive performance.

\section{Conclusion}

Our findings do not support the hypothesis that greater carotid CWS negatively impacts cognitive performance, at least not among relatively highly educated individuals in late middle age with adequately controlled cardiovascular risk factors. 


\section{Introduction}

Cognitive impairment and dementia are among the most feared conditions of old age ${ }^{1}$ and their prevalence, with the ageing of the population, continues to grow. ${ }^{2}$ Despite intensive research efforts, the mechanisms underlying age-related deterioration in cognitive performance remain incompletely understood. Vascular factors play an important role ${ }^{3}$ and may include structural alterations of the carotid arteries, the main suppliers of blood to the brain.

As the vasculature ages, ${ }^{4}$ but also in response to haemodynamic and atherosclerotic stimuli, ${ }^{5}$ carotid arteries undergo structural changes. This process of structural changes is known as arterial remodelling and is characterized by diameter widening and wall thickening, ${ }^{5-7}$ resulting from a complex interplay between vasoactive molecules, extracellular matrix turnover, and inflammatory activity. ${ }^{5,6}$ It is thought that arterial remodelling aims at normalising circumferential wall stress (CWS). ${ }^{5}$ Arterial remodelling is, however, not a uniform process ${ }^{5-7}$ and can be maladaptive, resulting in greater CWS, as is, for example, the case in diabetes. ${ }^{5}$

In theory, there are at least two mechanisms through which carotid CWS might influence cognitive performance. First, greater CWS has been related to the risk of plaque fissuring ${ }^{8}$ and rupture $^{9}$ with consequential (micro)embolisation of plaque debris to the brain. Cerebral emboli cause neuronal ischaemia, which can ultimately lead to neuronal dysfunction and cell death. Alternatively, or simultaneously, greater CWS may induce endothelial dysfunction, ${ }^{10}$ which at the level of the downstream microcirculation can contribute to blood-brain barrier disruption. Such disruption has been suggested to precipitate cerebral small vessel disease. ${ }^{11}$ Collectively, CWS may thus be linked to cognitive performance through vascular damage of the brain. Indeed, previous studies have shown that circumferential wall tension ${ }^{12}$ and stress ${ }^{13}$ are associated with a greater prevalence of silent lacunar infarcts ${ }^{12}$ and a greater severity of white matter hyperintensities. ${ }^{13}$ To date, however, data on the association of CWS with cognitive performance are lacking.

In the present study, we therefore tested the hypothesis that greater carotid CWS is associated with impairments of cognitive performance. We additionally evaluated whether such an association, if present, differs by diabetes status, not only because type 2 diabetes is associated with maladaptive carotid remodelling ${ }^{5}$ and cognitive impairment, ${ }^{14}$ but also because diabetes affects plaque composition, ${ }^{15}$ which increases plaque vulnerability. 


\section{Methods}

\section{Study population}

In this study, we used data from The Maastricht Study, an observational prospective population-based cohort study enriched with individuals with type 2 diabetes. The rationale and design have been described previously. ${ }^{16}$ In brief, the study focuses on the aetiology, pathophysiology, complications, and comorbidities of type 2 diabetes and is characterised by an extensive phenotyping approach. All individuals aged between 40 and 75 years living in the southern part of the Netherlands and sufficiently proficient in the Dutch language were eligible for participation. Participants were recruited through mass media campaigns and from the municipal registries and the regional Diabetes Patient Registry via mailings. For reasons of efficiency, recruitment was stratified according to known type 2 diabetes status.

For the present study, cross-sectional data from the first 866 participants were used who completed the baseline survey between November 2010 and March 2012. Each participant underwent all examinations within a time window of three months. We excluded participants with type 1 diabetes $(n=4)$, as well as those with missing data $(n=140)$ on cognitive performance $(n=21)$, CWS $(n=53)$, and/or one or more of the potential confounders $(n=80)$. The Maastricht Study has been approved by the institutional medical ethical committee (NL31329.068.10) and the Netherlands Health Council under the Dutch "Law for Population Studies" (Permit 131088-105234-PG). All participants gave written informed consent.

\section{Cognitive assessment}

All individuals completed a concise (30 $\mathrm{min}$ ) neuropsychological test battery to assess cognitive performance. ${ }^{16}$ For conceptual clarity and to increase the robustness of the underlying cognitive construct, test scores were standardised and divided into three cognitive domains (ie, memory function, executive function and attention, and information processing speed), as detailed in the Supplement. In short, memory function was evaluated using the Verbal Learning Test by averaging total immediate and delayed recall scores. The composite score for information processing speed was derived from the Stroop Colour Word Test Part I and II, the Concept Shifting Test Part A and B, and the Letter-Digit Substitution Test. Executive function and attention was assessed by the Stroop Colour Word Test Part III and the Concept Shifting Test Part C. Where necessary, individual test scores were inverted so that higher scores indicated better cognitive performance. 


\section{Circumferential wall stress}

Carotid ultrasound examinations were performed by trained vascular technicians who were unaware of the participants' clinical characteristics. Measurements took place in a dark, quiet, and temperature-controlled room $\left(21-23^{\circ} \mathrm{C}\right)$ and were performed in supine position after a resting period of $10 \mathrm{~min}$. Talking or sleeping was not allowed during the examination. Structural properties of the left carotid artery (at least $10 \mathrm{~mm}$ proximal to the carotid bulb) were determined with use of an ultrasound scanner equipped with a 7.5-MHz linear probe (MyLab 70, Esaote Europe, Maastricht, the Netherlands). This setup enabled the measurement of intima-media thickness (IMT) and interadventitial diameter (IAD), as described in more detail elsewhere. ${ }^{17,18}$

Briefly, based on radio frequency multiple M-line analysis, mean IMT and IAD were calculated along a $18.24 \mathrm{~mm}$ plaque-free segment of the left common carotid artery during end-diastole. IMT was defined as the distance between the lumen-intima and media-adventitia interfaces of the far (posterior) wall. IAD was defined as the distance between the media-adventitia interfaces of the near and far wall. The median IMT and $I A D$ of three consecutive measurements were used in the analyses.

From IMT and IAD, lumen diameter (LD) was calculated using the following formula: $L D=I A D-(2 * I M T)$ in $\mathrm{mm}^{5}$ CWS was then calculated according to Laplace's law as $P^{*}(r / w)$, where $P$ is transmural pressure, $r$ is lumen radius and $w$ is wall thickness. For the present study, both pulse pressure (PP) and mean arterial pressure (MAP) were used as representatives of transmural pressure in order to obtain a measure of pulsatile $\left(\mathrm{CWS}_{\text {pulsatile }}\right)$ and average ( $\left.\mathrm{CWS}_{\text {mean }}\right)$ mechanical load on the vessel wall. Local carotid PP was obtained from carotid pressure waveform calibration as specified in the Supplement. Brachial MAP was measured repeatedly during the vascular assessment at a 5-min interval with use of a commercially validated oscillometric device (Accutorr Plus, Datascope Inc., Montvale, NJ, USA), and the average of these measurements was taken. With PP and MAP as representatives of transmural pressure, $\mathrm{CWS}_{\text {pulsatile }}$ and $\mathrm{CWS}_{\text {mean }}$ were calculated as $(\mathrm{PP} *(\mathrm{LD} / 2)) / \mathrm{IMT}$ and $(\mathrm{MAP} *(\mathrm{LD} / 2)) / \mathrm{IMT}$, respectively, and expressed in $\mathrm{kPa}$.

Reproducibility was assessed in 12 individuals ( 6 men; $60.8 \pm 6.8$ years; 6 with type 2 diabetes) who were examined by two observers at two occasions spaced one week apart. The intra- and inter-observer intra-class correlation coefficients were, respectively, 0.90 and 0.91 for IMT, and 0.98 and 0.95 for IAD. 


\section{Covariates}

As described in more detail elsewhere ${ }^{16}$ diabetes status was ascertained by an oral glucose tolerance test in those not treated with insulin, and defined according to the $2006 \mathrm{WHO}^{19}$ diagnostic criteria. Participants were also considered to have type 2 diabetes if they were prescribed glucose-lowering medication without a prior diagnosis of type 1 diabetes. Information on alcohol consumption, smoking behaviour (never/ former/current), and prior cardiovascular disease (CVD) was collected from web-based questionnaires. ${ }^{16}$ Frequency of alcohol consumption was categorised as never, low (1-7 glasses per week for women, 1-14 glasses for men), or high (>7 glasses per week for women, $>14$ for men). ${ }^{16}$ Prior CVD was defined as a history of myocardial infarction, stroke, or vascular surgery (including angioplasty) of coronary, carotid, abdominal aortic, or peripheral arteries. ${ }^{16}$ Hypertension was defined as a systolic blood pressure $\geq 140$ $\mathrm{mmHg}$ (based on office blood pressure measurements ${ }^{16}$ ), a diastolic blood pressure $\geq$ $90 \mathrm{mmHg}$, and/or use of antihypertensive medication. Medication use was determined as described previously. ${ }^{16}$ An automatic analyser (Beckman Synchron LX20, Beckman Coulter Inc., Brea, USA) was used to measure fasting serum concentrations of total cholesterol, high-density lipoprotein (HDL) cholesterol, triglycerides, and creatinine. Cystatin $\mathrm{C}$ was measured by a particle enhanced immunoturbidimetric assay (Roche Cobas 8000, Roche Diagnostics, Basel, Switzerland). Glomerular filtration rate (eGFR) was estimated from both serum creatinine and cystatin $C$ concentrations using the Chronic Kidney Disease Epidemiology Collaboration (CKD-EPI) equation. ${ }^{20}$ Body Mass Index (BMI) was calculated as body mass $(\mathrm{kg})$ divided by height $(\mathrm{m})$ squared. Educational level was derived from self-report and divided into low, intermediate, and high, as described in detail previously. ${ }^{21}$ The Mini International Neuropsychiatric Interview (MINI) was used to detect the presence of a current depression. ${ }^{16}$

\section{Statistical analyses}

All statistical analyses were performed with use of SPSS for Windows, version 21.0 (IBM SPSS, IBM Corp, Armonk, NY, USA). Variables with a skewed distribution (ie, $\mathrm{CWS}_{\text {pulsatile' }}$ Stroop Colour Word Test scores, Concept Shifting Test scores, and triglyceride concentrations) were transformed with the natural logarithm prior to analyses. A two-sided $p$-value $<0.05$ was considered statistically significant, except for interaction analyses where the significance level was set at 0.10 .

Multiple linear regression analyses were used to analyse the association between CWS and cognitive performance. These associations were adjusted for demographic characteristics (ie, age, sex, and educational level; model 1) and additionally for cardiovascular risk factors (ie, BMI, total cholesterol/HDL ratio, triglyceride concentration, use of lipid-modifying medication, hypertension, type 2 diabetes, eGFR, smoking, 
alcohol consumption, and prior CVD; model 2), as well as the presence of a current depression (model 3). To evaluate whether the association between CWS and cognitive performance differed by the presence or absence of type 2 diabetes, multiplicative interaction terms (ie, CWS pulsatile $^{*}$ type 2 diabetes and $\mathrm{CWS}_{\text {mean }}{ }^{*}$ type 2 diabetes) were constructed and incorporated in the regression models. Using a similar approach, we also tested for interaction with age and sex.

Several sensitivity analyses were performed. First, we explored the association between the individual components of CWS (ie, IMT, IAD, PP and MAP) and cognitive performance. Second, we evaluated whether the lumen-to-wall ratio, calculated as LD/(2*IMT), was associated with cognitive performance, as this measure provides information on the geometric aspects of CWS. Finally, we explored the potential consequences of the complete case analysis approach we chose as the primary analysis. To this end, we reanalysed the data with inclusion of individuals excluded from the primary analyses because of missing data on covariates. In these final analyses, only demographical factors were considered as covariates.

\section{Results}

Demographic and clinical characteristics of the 722 individuals available for analysis are shown in Table 4.1, stratified according to tertiles of CWS $_{\text {pulsatile }}$. Overall, participants were of late middle age (mean age $60 \pm 8$ years) and slightly more likely to be male (55.1\%). Note that although in general the cardiovascular risk profile worsened with increasing CWS, cardiovascular risk factors were generally adequately controlled (Table 4.1). Characteristics of individuals excluded from the analyses due to incomplete data ( $n=140 ; 16 \%)$ are presented in Table S4.1 (Supplement). In brief, those excluded more often had type 2 diabetes and more frequently suffered from a current depression. In addition, they performed slightly worse on tests of memory function.

\section{Circumferential wall stress and cognitive performance}

Table 4.2 shows the association between CWS and cognitive performance. After adjustment for age, sex, and educational level, neither $\mathrm{CWS}_{\text {pulsatile }}$ nor $\mathrm{CWS}_{\text {mean }}$ were statistically significantly associated with cognitive performance (all p-values $\geq 0.31$; model 1). Additional adjustments for cardiovascular risk factors and current depression did not materially change these results (models 2 and 3).

Multiplicative interaction analyses indicated that the null association between CWS and cognitive performance did not differ by age ( $p$-values for interaction $\geq 0.15$; model 1 ) or sex $(\geq 0.16$; model 1$)$. In contrast, statistically significant interaction was observed between 


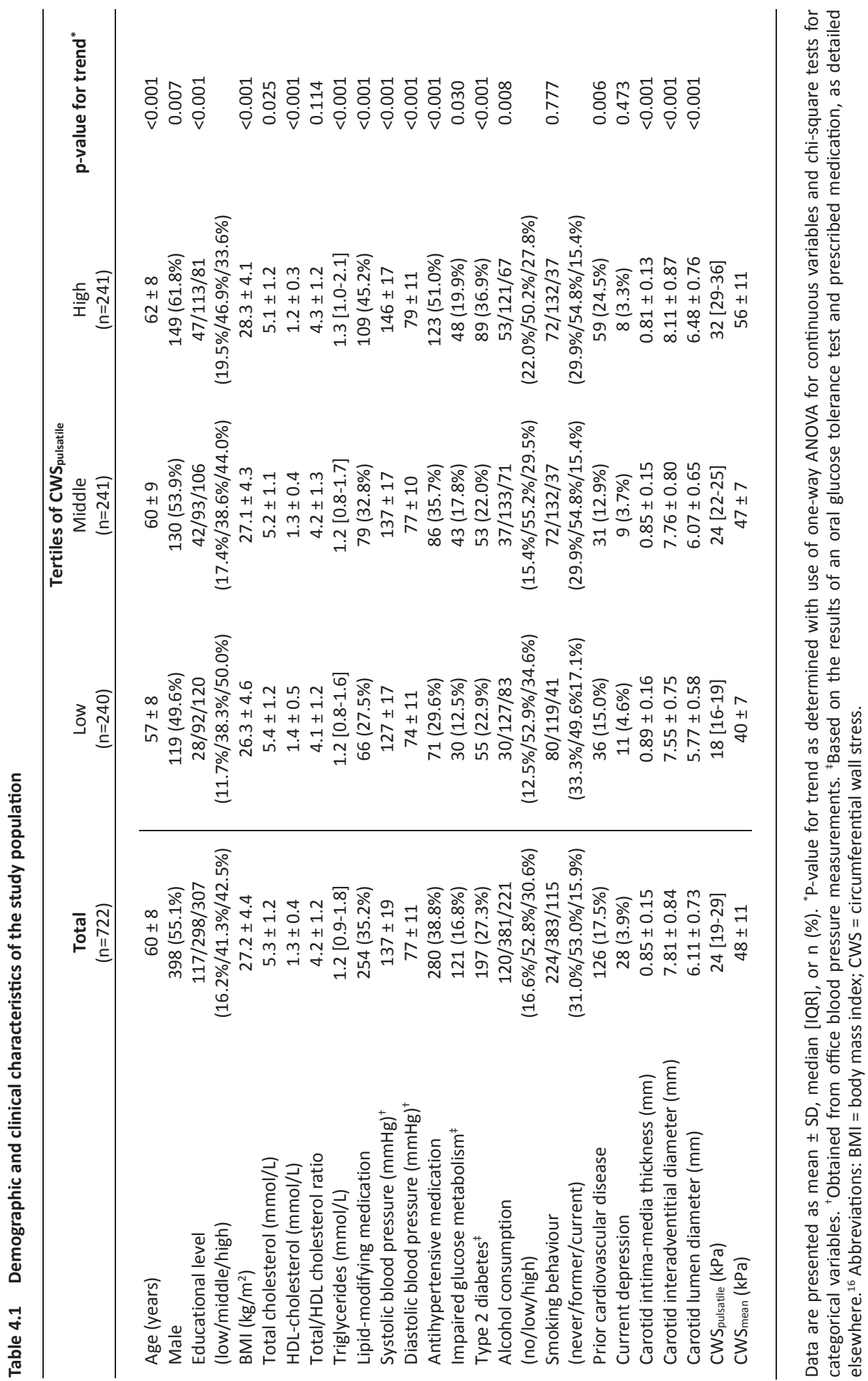


CWS and diabetes status for memory function ( $p$-values for interaction $0.04\left(\right.$ CWS $_{\text {pulsatile }}$ ) and 0.02 ( CWS $\left._{\text {mean }}\right)$; model 1). Subsequent stratified analysis, however, revealed that there were no statistically significant association between CWS (ie, CWS $\mathrm{Culsatile}_{\text {and }}$ $\left.\mathrm{CWS}_{\text {mean }}\right)$ and memory performance among individuals with type 2 diabetes, nor among those without diabetes (Table S4.2, Supplement).

\section{Sensitivity analyses}

When the individual components of CWS were considered, none of these (ie, IMT, IAD, $L D, P P$, and MAP) was associated with performance on any of the cognitive domains examined (Table S4.3, Supplement). Similarly, the lumen-to-wall ratio was unrelated to cognitive performance (Table S4.3, Supplement).

Reanalyses of the data with inclusion of individuals excluded from the primary analyses because of missing data on the potential confounders $(n=66)$ showed, in this larger study population ( $n=788)$, similar null associations between CWS and cognitive performance in age-, sex-, and educational level-adjusted models (Table S4.4, Supplement).

Table 4.2 Associations between circumferential wall stress and cognitive performance

\begin{tabular}{lccc}
\hline & Memory & Processing speed & $\begin{array}{c}\text { Executive function \& } \\
\text { attention }\end{array}$ \\
\hline CWS pulsatile & -0.014 & & \\
Model 1 & $(-0.078$ to 0.051$)$ & -0.024 & -0.034 \\
Model 2 & -0.001 & $(-0.088$ to 0.040$)$ & -0.026 \\
Model 3 & $(-0.066$ to 0.065$)$ & $(-0.092$ to 0.040$)$ & -0.019 \\
& -0.001 & -0.027 & $(-0.088$ to 0.050$)$ \\
CWS & $(-0.067$ to 0.064$)$ & $(-0.093$ to 0.039$)$ & -0.019 \\
Model 1 & & & $(-0.088$ to 0.050$)$ \\
Model 2 & -0.001 & -0.014 & -0.028 \\
& $(-0.065$ to 0.063$)$ & $(-0.078$ to 0.050$)$ & $(-0.095$ to 0.038$)$ \\
Model 3 & -0.005 & -0.031 & -0.033 \\
& $(-0.070$ to 0.060$)$ & $(-0.096$ to 0.035$)$ & $(-0.102$ to 0.035$)$ \\
& -0.005 & -0.031 & -0.033 \\
\hline
\end{tabular}

$\mathrm{N}=722$. Data are presented as standardised regression coefficient ( $95 \%$ confidence interval), which reflect the change in cognitive performance per standard deviation increase in pulsatile ( $\left.\mathrm{CWS}_{\text {pulsatile }}\right)$ or mean ( $\left.\mathrm{CWS}_{\text {mean }}\right)$ circumferential wall stress. CWS pulsatile was transformed with the natural logarithm prior to analysis. Model 1: adjusted for age, sex, and educational level; Model 2: additional adjustment for body mass index, total/high density lipoprotein-cholesterol ratio, triglycerides, use of lipid-modifying medication, hypertension, presence of type 2 diabetes, estimated glomerular filtration rate, smoking behaviour, alcohol consumption, and history of cardiovascular disease(s); Model 3: additional adjustment for the presence of a current depression. Abbreviations: CWS = circumferential wall stress. 


\section{Discussion}

The present study shows that, in a population-based cohort of individuals in late middle age and enriched for type 2 diabetes, carotid circumferential wall stress is not associated with cognitive performance. This null association was observed in both individuals with and without diabetes, regardless of age and sex. Collectively, our findings do not support our hypothesis that greater CWS negatively impacts cognitive performance, at least not among relatively highly educated individuals in late middle age with adequately controlled cardiovascular risk factors.

To our knowledge, this is the first study to investigate the association between CWS and cognitive performance. Previous studies, although very limited in number, have explored associations of greater CWS with brain structure and showed that the prevalence and severity of cerebral small vessel disease increases with greater CWS. ${ }^{12,13}$ Cerebral small vessel disease, in turn, is implicated in (vascular) dementia and has been associated with general cognitive performance. ${ }^{22}$ It is, therefore, somewhat surprising that we did not observe any association of CWS with cognitive performance. This apparent discrepancy may be explained by the concept of cognitive reserve, which reflects the brain's ability to withstand neuropathology before performance is affected. ${ }^{23}$ As such, it is theoretically possible that CWS does induce vascular brain damage, but because the brain is able to compensate for a certain degree of pathology, structural changes do not necessarily affect cognitive performance.

Although our findings suggest that CWS is unrelated to cognitive performance at a population level, they do not preclude the possibility that such an association is present in individuals with a lower cognitive reserve capacity or may become apparent with increasing age or accumulating (vascular) brain pathology. More specifically, it may be the balance between cognitive reserve and severity of (age-related) structural brain damage that determines whether greater CWS impairs cognitive performance. At first sight, a somewhat counterintuitive observation in this regard is that the null association between CWS and cognitive performance did not differ by age and was similar in individuals with and without type 2 diabetes, while cerebral small vessel disease is considered an age-related phenomenon that is exacerbated by diabetes. ${ }^{24}$ Note, however, that the educational level, and thus cognitive reserve capacity, of the study population was overall high (ie, $42.5 \%$ of the individuals had higher vocational education or went to university). Combined with the exclusion of older individuals (ie, $\geq 75$ years) and the fact that cardiovascular risk factors were generally adequately controlled in the study population, this may have limited our ability to detect a potential association between CWS and cognitive performance with increasing age. Likewise, the lack of a modulating 
effect of diabetes status should be viewed in light of the overall good glycaemic control of individuals with type 2 diabetes in our study population (mean HbA1c $6.9 \pm 0.8 \%$ ).

Previous studies on carotid artery structure and its associations with cognitive performance have focused only on one aspect of structure, namely IMT, which is often considered an early marker of atherosclerosis. ${ }^{25}$ At first glance, our finding that IMT was not associated with any of the cognitive domains assessed seems to be in contrast with most, but not all, ${ }^{26-29}$ previous studies reporting that IMT is related to worse cognitive performance, accelerated cognitive decline, and an increased risk of dementia. ${ }^{30-49}$ The majority of these studies ${ }^{30-35,37-40,44,45,47,49} \mathrm{did}$, however, not specify whether IMT measurements were performed at a plaque-free site and may therefore have explored the cerebral effects of more severe stages of carotid atherosclerosis, especially when comparing highest to lowest quintiles of IMT. ${ }^{30,31,35}$ In line with this suggestion, several studies included IMT measurements from the internal carotid artery ${ }^{31-33,44,45,47,48}$ or carotid bifurcation, ${ }^{31,32,39,44,45,48}$ ie, locations that are more prone to atherosclerosis. ${ }^{50}$ Interestingly in this respect, and in line with our results, a closer examination of data from the Framingham ${ }^{47}$ and Troms $\varnothing^{48}$ studies indicated that internal, but not common, carotid IMT is associated with cognitive dysfunction.

Our findings on IMT may imply that early stages of carotid atherosclerosis do not impact cognitive function, which seems plausible as mild abnormalities are unlikely to substantially alter cerebral haemodynamics. In addition, cerebral microemboli are considered to originate mainly from (unstable) carotid plaques and are more frequent with increasing severity of carotid stenosis. ${ }^{51}$ The concept that cognitive performance declines with the severity of carotid atherosclerosis is corroborated by studies ${ }^{46,48}$ in which associations with cognition were stronger for the presence of carotid plaque than for carotid IMT. In line with this, data from the Framingham study indicated that severe stenosis ( $\geq 50 \%$ ) is associated with poorer cognitive performance, whereas milder stenosis $(\geq 25 \%)$ is not. ${ }^{47}$ The IMT as measured in the present study, with a mean value of 0.85 $\mathrm{mm}$, may in part however reflect adaptive remodelling in response to haemodynamic changes rather than atherosclerosis per se.

Our novel findings should be viewed in light of the following limitations. First, excluding individuals with missing data may have resulted in selection of individuals less likely to have diabetes and with higher cognitive performance test scores (Table S4.1, Supplement). Nonetheless, we show that our complete case analysis approach did not distort the results because similar null associations were observed when individuals with missing data on the covariates were included in model 1 (Table S4.4, Supplement). A second, but related, limitation concerns the generalisability of our findings. As noted 


\section{Chapter 4}

above, our study population consisted of individuals endowed with ample cognitive reserve, not only because they were highly educated but also because individuals aged $\geq$ 75 were excluded from participation in The Maastricht Study. Consequently, we cannot exclude the possibility that CWS is associated with cognitive performance in the absence of such compensatory reserve capacity. On the other hand, the CWS observed in the present study is comparable or only slightly lower to that reported in other studies, ${ }^{13}$, 52,53 which makes it unlikely that our null associations are simply explained by exposure to relatively low CWS. Third, data on brain structure were not available and we were therefore unable to confirm or refute previous findings that CWS is associated with cerebral small vessel disease. A notable strength of our study is the cognitive test battery used, which was constructed to assess cognitive performance across multiple domains and had the ability to detect even subtle impairments in cognitive performance.

In conclusion, our findings suggest that carotid CWS is unrelated to cognitive performance and thereby indicate that maladaptive carotid remodelling does not directly affect brain function, at least not in individuals in late middle age with presumably ample cognitive reserves. As this is the first study to evaluate the association between CWS and cognitive performance, further research is clearly needed to verify our findings and their generalisability. Future longitudinal studies are required to include measures of both cognitive performance and vascular brain pathology in order to identify potential differential effects of greater CWS on brain structure and function. 


\section{References}

1. Alzheimer's Australia NSW. Addressing the Stigma associated with Dementia. 2010. Available from: https://nsw.fightdementia.org.au/sites/default/files/2010NSWAddressingStigma DiscussionPaper2.pdf. Last accessed July 23, 2015..

2. Ferri, C.P., et al., Global prevalence of dementia: a Delphi consensus study. Lancet, 2005. 366(9503): p. 2112-7.

3. Gorelick, P.B., et al., Vascular contributions to cognitive impairment and dementia: a statement for healthcare professionals from the american heart association/american stroke association. Stroke, 2011. 42(9): p. 2672-713.

4. Lakatta, Eg, and D. Levy, Arterial and cardiac aging: major shareholders in cardiovascular disease enterprises: Part I: aging arteries: a "set up" for vascular disease. Circulation, 2003. 107(1): p. 139-46.

5. Henry, R.M., et al., Carotid arterial remodeling: a maladaptive phenomenon in type 2 diabetes but not in impaired glucose metabolism: the Hoorn study. Stroke, 2004. 35(3): p. 671-6.

6. Ward, M.R., et al., Arterial remodeling. Mechanisms and clinical implications. Circulation, 2000. 102(10): p. 1186-91.

7. Pasterkamp, G., P.F. Fitzgerald, and D.P. de Kleijn, Atherosclerotic expansive remodeled plaques: a wolf in sheep's clothing. J Vasc Res, 2002. 39(6): p. 514-23.

8. Richardson, P.D., M.J. Davies, and G.V. Born, Influence of plaque configuration and stress distribution on fissuring of coronary atherosclerotic plaques. Lancet, 1989. 2(8669): p. 941-4.

9. Cheng, G.C., et al., Distribution of circumferential stress in ruptured and stable atherosclerotic lesions. A structural analysis with histopathological correlation. Circulation, 1993. 87(4): p. 1179-87.

10. Kliche, K., et al., Role of cellular mechanics in the function and life span of vascular endothelium. Pflugers Arch, 2011. 462(2): p. 209-17.

11. Wardlaw, J.M., Blood-brain barrier and cerebral small vessel disease. J Neurol Sci, 2010. 299(1-2): p. 66-71.

12. Okada, Y., et al., Mechanical stresses, arterial stiffness, and brain small vessel diseases: Shimanami Health Promoting Program Study. Stroke, 2014. 45(11): p. 3287-92.

13. Brisset, M., et al., Large-vessel correlates of cerebral small-vessel disease. Neurology, 2013. 80(7): p. 662-9.

14. Biessels, G.J., et al., Dementia and cognitive decline in type 2 diabetes and prediabetic stages: towards targeted interventions. Lancet Diabetes Endocrinol, 2014. 2(3): p. 246-55.

15. Pasterkamp, G., Methods of accelerated atherosclerosis in diabetic patients. Heart, 2013. 99(10): p. 743-9.

16. Schram, M.T., et al., The Maastricht Study: an extensive phenotyping study on determinants of type 2 diabetes, its complications and its comorbidities. Eur J Epidemiol, 2014. 29(6): p. 439-51.

17. Hermeling, E., et al., The dicrotic notch as alternative time-reference point to measure local pulse wave velocity in the carotid artery by means of ultrasonography. J Hypertens, 2009. 27(10): p. 2028-35.

18. Willekes, C., et al., Evaluation of off-line automated intima-media thickness detection of the common carotid artery based on M-line signal processing. Ultrasound Med Biol, 1999. 25(1): p. 57-64.

19. World Health Organisation. Definition and diagnosis of diabetes mellitus and intermediate hyperglycemia: report of a WHO/IDF consultation. 2006. Available from: http://www.idf.org/ webdata/docs/WHO_IDF_definition_diagnosis_of_diabetes.pdf. Last accessed July 19, 2015.

20. Inker, L.A., et al., Estimating glomerular filtration rate from serum creatinine and cystatin $\mathrm{C}$. N Engl J Med, 2012. 367(1): p. 20-9.

21. Spauwen, P.J., et al., Both Low and High 24-Hour Diastolic Blood Pressure Are Associated With 
Worse Cognitive Performance in Type 2 Diabetes: The Maastricht Study. Diabetes Care, 2015. 38(8): p. 1473-80.

22. Van der Flier, W.M., et al., Small vessel disease and general cognitive function in nondisabled elderly: the LADIS study. Stroke, 2005. 36(10): p. 2116-20.

23. Stern, Y., et al., Exploring the neural basis of cognitive reserve. J Clin Exp Neuropsychol, 2003. 25(5): p. 691-701.

24. Pantoni, L., Cerebral small vessel disease: from pathogenesis and clinical characteristics to therapeutic challenges. Lancet Neurol, 2010. 9(7): p. 689-701.

25. Polak, J.F., Carotid intima-media thickness: an early marker of cardiovascular disease. Ultrasound Q, 2009. 25(2): p. 55-61.

26. Knopman, D., et al., Cardiovascular risk factors and cognitive decline in middle-aged adults. Neurology, 2001. 56(1): p. 42-8.

27. Johnston, S.C., et al., Cognitive impairment and decline are associated with carotid artery disease in patients without clinically evident cerebrovascular disease. Ann Intern Med, 2004. 140(4): p. 237-47.

28. Kohara, K., et al., Atherosclerotic indices for the prediction of cognitive impairment in a middle-aged to elderly general population: shimanami health promoting program study. J Am Geriatr Soc, 2012. 60(10): p. 1996-7.

29. Lopez-Oloriz, J., et al., Asymptomatic cervicocerebral atherosclerosis, intracranial vascular resistance and cognition: the AsIA-neuropsychology study. Atherosclerosis, 2013. 230(2): p. 330-5.

30. Van Oijen, M., et al., Atherosclerosis and risk for dementia. Ann Neurol, 2007. 61(5): p. 40310.

31. Cerhan, J.R., et al., Correlates of cognitive function in middle-aged adults. Atherosclerosis Risk in Communities (ARIC) Study Investigators. Gerontology, 1998. 44(2): p. 95-105.

32. Carlsson, C.M., et al., Increased atherogenic lipoproteins are associated with cognitive impairment: effects of statins and subclinical atherosclerosis. Alzheimer Dis Assoc Disord, 2009. 23(1): p. 11-7.

33. Haan, M.N., et al., The role of APOE epsilon4 in modulating effects of other risk factors for cognitive decline in elderly persons. JAMA, 1999. 282(1): p. 40-6.

34. Sander, K., et al., Carotid- intima media thickness is independently associated with cognitive decline. The INVADE study. Int J Geriatr Psychiatry, 2010. 25(4): p. 389-94.

35. Crystal, H.A., et al., Associations of cardiovascular variables and HAART with cognition in middle-aged HIV-infected and uninfected women. J Neurovirol, 2011. 17(5): p. 469-76.

36. Dias Eda, M., et al., Carotid intima-media thickness is associated with cognitive deficiency in hypertensive patients with elevated central systolic blood pressure. Cardiovasc Ultrasound, 2012. 10: p. 41.

37. Gatto, N.M., et al., Subclinical atherosclerosis is weakly associated with lower cognitive function in healthy hyperhomocysteinemic adults without clinical cardiovascular disease. Int J Geriatr Psychiatry, 2009. 24(4): p. 390-9.

38. Haley, A.P., et al., Carotid artery intima-media thickness and cognition in cardiovascular disease. Int J Cardiol, 2007. 121(2): p. 148-54.

39. Komulainen, P., et al., Carotid intima-media thickness and cognitive function in elderly women: a population-based study. Neuroepidemiology, 2007. 28(4): p. 207-13.

40. Reijmer, Y.D., et al., The metabolic syndrome, atherosclerosis and cognitive functioning in a non-demented population: the Hoorn Study. Atherosclerosis, 2011. 219(2): p. 839-45.

41. Silvestrini, M., et al., Carotid atherosclerosis and cognitive decline in patients with Alzheimer's disease. Neurobiol Aging, 2009. 30(8): p. 1177-83.

42. Wendell, C.R., et al., Carotid intimal medial thickness predicts cognitive decline among adults without clinical vascular disease. Stroke, 2009. 40(10): p. 3180-5.

43. Xiang, J., et al., Carotid artery atherosclerosis is correlated with cognitive impairment in an 
elderly urban Chinese non-stroke population. J Clin Neurosci, 2013. 20(11): p. 1571-5.

44. Zhong, W., et al., Carotid atherosclerosis and 10-year changes in cognitive function. Atherosclerosis, 2012. 224(2): p. 506-10.

45. Zhong, W., et al., Carotid atherosclerosis and cognitive function in midlife: the Beaver Dam Offspring Study. Atherosclerosis, 2011. 219(1): p. 330-3.

46. Auperin, A., et al., Ultrasonographic assessment of carotid wall characteristics and cognitive functions in a community sample of 59- to 71-year-olds. The EVA Study Group. Stroke, 1996. 27(8): p. 1290-5.

47. Romero, J.R., et al., Carotid artery atherosclerosis, MRI indices of brain ischemia, aging, and cognitive impairment: the Framingham study. Stroke, 2009. 40(5): p. 1590-6.

48. Arntzen, K.A., et al., Carotid atherosclerosis predicts lower cognitive test results: a 7-year follow-up study of 4,371 stroke-free subjects - the Tromso study. Cerebrovasc Dis, 2012. 33(2): p. 159-65.

49. Zeki Al Hazzouri, A., et al., Intima-Media Thickness and Cognitive Function in Stroke-Free Middle-Aged Adults: Findings From the Coronary Artery Risk Development in Young Adults Study. Stroke, 2015. 46(8): p. 2190-6.

50. Kiechl, S. and J. Willeit, The natural course of atherosclerosis. Part I: incidence and progression. Arterioscler Thromb Vasc Biol, 1999. 19(6): p. 1484-90.

51. Zhang, C., et al., Microembolic signals and carotid plaque characteristics in patients with asymptomatic carotid stenosis. Scand Cardiovasc J, 2009. 43(5): p. 345-51.

52. Beijers, H.J., et al., Metabolic syndrome in nondiabetic individuals associated with maladaptive carotid remodeling: the Hoorn Study. Am J Hypertens, 2011. 24(4): p. 429-36.

53. Ferreira, I., et al., Clustering of metabolic syndrome traits is associated with maladaptive carotid remodeling and stiffening: a 6-year longitudinal study. Hypertension, 2012. 60(2): p. $542-9$. 


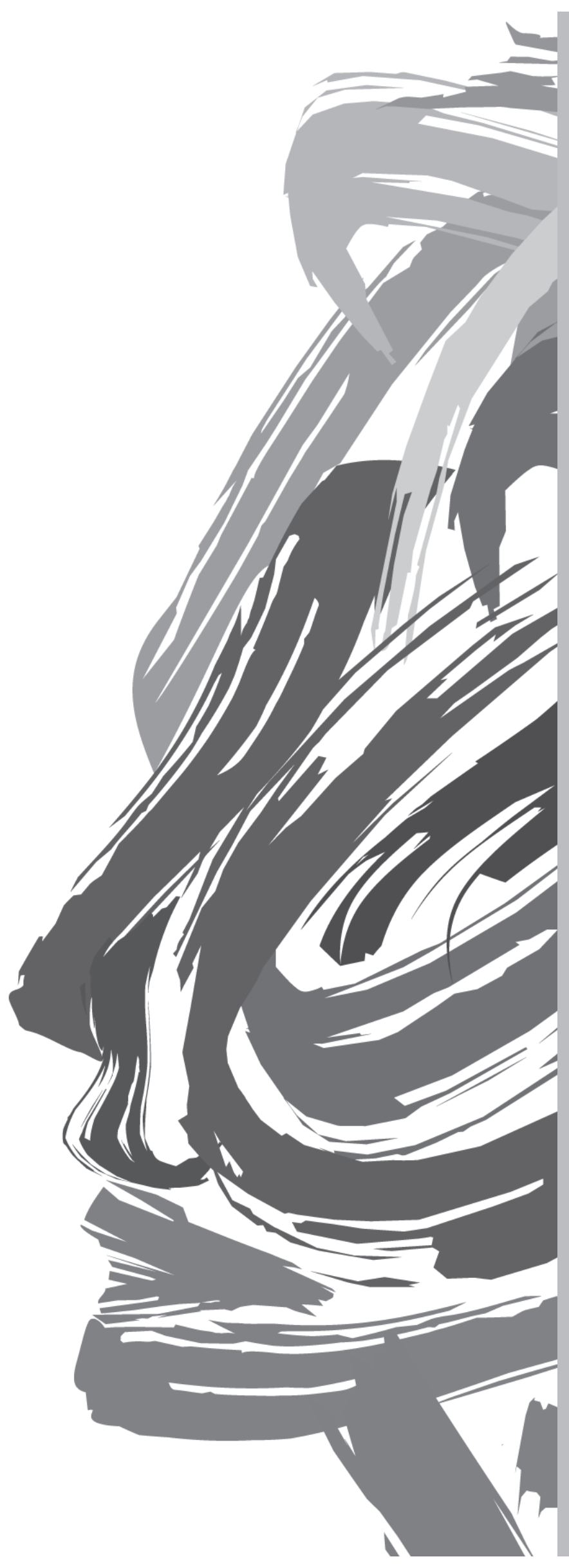


Supplement to Chapter 4 


\section{Extended methods}

\section{Cognitive assessment}

The composite memory score was derived from the Verbal Learning Test by weighting total immediate and delayed recall scores. The domain processing speed included the Stroop Colour Word Test Part I and II, the Concept Shifting Test Part A and B, and the Letter-Digit Substitution Test. Executive function and attention was assessed by the Stroop Colour Word Test Part III and the Concept Shifting Test Part C. A brief description of the individual tests is provided below.

Raw test scores were transformed into z-scores. Standardised scores of the Stroop Colour Word Test and Concept Shifting Test were inverted so that higher scores indicated better cognitive performance. Thereafter, domain-specific scores were calculated by averaging the $z$-scores from (sub)tests within that domain (eg, memory $=$ z-score ${ }_{\text {immediate }}$ recall $+z$-score dealyed recall $/ 2$ ).

Description of the individual cognitive tests used in the present study:

\section{Verbal Learning Test $^{1}$}

Fifteen unrelated, monosyllabic, words were presented on a computer screen in five subsequent trials. After each trial, participants were instructed to recall as many words as possible in any order. Twenty minutes after the last trial, participants were asked again to reproduce the words. Outcomes recorded included the total number of words correctly recalled over the five trials (total immediate recall) and the number of correctly recalled words during delayed recall (delayed recall).

\section{Stroop Colour Word Test ${ }^{2}$}

In this test, that consisted of three parts, participants were firstly asked to read aloud colour names (ie, red, blue, yellow, and green) that were printed in black ink (Part I). Secondly, they were instructed to name solid colour patches (Part II). Finally, participants had to name the ink colour of colour names that were printed in an incongruent colour (eg, participants were asked to say red when the word yellow was printed in red) (Part III). The time needed to complete Part III was adjusted for the average time needed to complete Part I and II.

\section{Concept Shifting Test ${ }^{3}$}

This test, a modification of the Trailing Making Test, consisted of four subtasks. During each subtask, participants were shown 16 small circles aligned along a larger imaginary circle. The small circles contained (a combination of) digits, letters, or were empty. 
Participants were instructed to cross-out as quickly as possible the digits in ascending order (Part A), the letters in alphabetic order (Part B), and the letters and digits in alternating order (Part C). Thereafter, participants were asked to cross-out empty circles in a clockwise fashion in two consecutive trials (Part 0 ). In this way, test results could be accounted for basic motor speed. The time needed to complete subtasks A and B was adjusted for the average time needed to complete Part 0 , the time needed to completed Part $\mathrm{C}$ for the average time of Part $\mathrm{A}$ and $\mathrm{B}$.

\section{Letter-Digit Substitution Test ${ }^{4}$}

Participants were requested to match digits to letters according to a given key. This key included the numbers 1 to 9 , each paired with a different letter. The outcome of interest was the number of correct substitutions within 90 seconds.

\section{Local carotid pulse pressure}

Pulse pressure at the carotid artery was calculated by calibrating the systolic-diastolic amplitude of the carotid artery tonometry waveform (sys-dias) tono $_{\text {o }}$ to pressure, assuming a constant difference between mean arterial pressure (MAP) and diastolic pressure (DP) along the large arteries: PPcar $=(\text { sys-dias })_{\text {tono }} *(\mathrm{MAP}-\mathrm{DP})_{\text {brach }} /(\text { mean-dias })_{\text {tono }}$. Tonometry waveforms were obtained from the right common carotid artery using the SphygmoCor (Atcor Medical, Sydney, Australia).

This procedure is the same as those described by Kelly and Fitchett ${ }^{5}$ and Van Bortel et al., ${ }^{6}$ except that the MAP and DP values at the brachial artery were taken as the respective averages over the vascular measurements (ie, over a 30 to 45 min period) as obtained with a validated commercial oscillometric device (Accutorr Plus, Datascope Inc., Montvale, NJ, USA). Cited procedures considered the mean-diastolic difference as obtained from a measured brachial artery pressure or diameter waveform.

The calibration approach used in the present study was chosen because of its practical applicability in a large-scale population-based study. We acknowledge that there are other methods to determine local carotid pulse pressure non-invasively, which include, for example, rescaling carotid distension waveforms with use of brachial distension waveforms, rescaling carotid tonometry waveforms with use of brachial mean arterial pressure obtained from brachial tonometry, and calculating carotid pressure from radial tonometry with use of a transfer function. Given the lively debate regarding the reliability and validity of the different methods available, ${ }^{7-9}$ we believe that a consensus on the best method remains elusive. 


\section{References}

1. Van der Elst, W., et al., Rey's verbal learning test: normative data for 1855 healthy participants aged 24-81 years and the influence of age, sex, education, and mode of presentation. J Int Neuropsychol Soc, 2005. 11(3): p. 290-302.

2. Van der Elst, W., et al., The Stroop color-word test: influence of age, sex, and education; and normative data for a large sample across the adult age range. Assessment, 2006. 13(1): p. 62 79.

3. Van der Elst, W., et al., The Concept Shifting Test: adult normative data. Psychol Assess, 2006. 18(4): p. 424-32.

4. Van der Elst, W., et al., The Letter Digit Substitution Test: normative data for 1,858 healthy participants aged 24-81 from the Maastricht Aging Study (MAAS): influence of age, education, and sex. J Clin Exp Neuropsychol, 2006. 28(6): p. 998-1009.

5. Kelly, R. and D. Fitchett, Noninvasive determination of aortic input impedance and external left ventricular power output: a validation and repeatability study of a new technique. J Am Coll Cardiol, 1992. 20(4): p. 952-63.

6. Van Bortel, L.M., et al., Non-invasive assessment of local arterial pulse pressure: comparison of applanation tonometry and echo-tracking. J Hypertens, 2001. 19(6): p. 1037-44.

7. Verbeke, F., et al., Noninvasive assessment of local pulse pressure: importance of brachial-toradial pressure amplification. Hypertension, 2005. 46(1): p. 244-8.

8. Adji, A. and M.F. O'Rourke, Brachial artery tonometry and the Popeye phenomenon: explanation of anomalies in generating central from upper limb pressure waveforms. J Hypertens, 2012. 30(8): p. 1540-51.

9. Lehmann, E.D., Regarding the accuracy of generalized transfer functions for estimating central aortic blood pressure when calibrated non-invasively. J Hypertens, 2000. 18(3): p. 347-49; author reply 349-50.

10. Schram, M.T., et al., The Maastricht Study: an extensive phenotyping study on determinants of type 2 diabetes, its complications and its comorbidities. Eur J Epidemiol, 2014. 29(6): p. 439-51. 
Table S4.1 Characteristics of individuals included and those excluded from the main analyses

\begin{tabular}{|c|c|c|c|c|}
\hline & $\begin{array}{c}\text { Included } \\
(n=722)\end{array}$ & $\begin{array}{c}\text { Excluded } \\
\left(n_{\max }=140\right)\end{array}$ & Missing* & $\begin{array}{c}\text { p- } \\
\text { value }\end{array}$ \\
\hline Age (years) & $60 \pm 8$ & $61 \pm 9$ & 0 & 0.126 \\
\hline Male & 398 (55.1 \%) & $74(52.9 \%)$ & 0 & 0.622 \\
\hline Educational level & $117 / 298 / 307$ & $27 / 57 / 56$ & 0 & 0.653 \\
\hline (low/middle/high) & $(16.2 \% / 41.3 \% / 42.5 \%)$ & $(19.3 \% / 40.7 \% / 40.0 \%)$ & & \\
\hline BMI $\left(\mathrm{kg} / \mathrm{m}^{2}\right)$ & $27.2 \pm 4.4$ & $27.9 \pm 5.1$ & 1 & 0.127 \\
\hline Total cholesterol (mmol/L) & $5.3 \pm 1.2$ & $5.0 \pm 1.2$ & 7 & 0.009 \\
\hline HDL-cholesterol (mmol/L) & $1.3 \pm 0.4$ & $1.3 \pm 0.5$ & 7 & 0.431 \\
\hline Total/HDL cholesterol ratio & $4.2 \pm 1.2$ & $4.2 \pm 1.3$ & 8 & 0.703 \\
\hline Triglycerides (mmol/L) & $1.2[0.9-1.8]$ & 1.4 [0.9-1.9] & 7 & 0.174 \\
\hline Lipid lowering medication & $254(35.2 \%)$ & $64(45.7 \%)$ & 0 & 0.018 \\
\hline SBP $(\mathrm{mmHg})^{\ddagger}$ & $137 \pm 19$ & $140 \pm 20$ & 2 & 0.081 \\
\hline $\mathrm{DBP}(\mathrm{mmHg})^{\ddagger}$ & $77 \pm 11$ & $77 \pm 9$ & 2 & 0.515 \\
\hline Antihypertensive medication & $280(38.8 \%)$ & $66(47.1 \%)$ & 0 & 0.065 \\
\hline Impaired glucose metabolism ${ }^{\S}$ & $121(16.8 \%)$ & $19(13.6 \%)$ & 0 & 0.349 \\
\hline Type 2 diabetes ${ }^{\S}$ & $197(27.3 \%)$ & $56(40.0 \%)$ & 0 & 0.002 \\
\hline Alcohol consumption & $120 / 381 / 221$ & $29 / 61 / 30$ & 20 & 0.107 \\
\hline (no/low/high) & $(16.6 \% / 52.8 \% / 30.6 \%)$ & $(24.2 \% / 50.8 \% / 25.0 \%)$ & & \\
\hline Smoking behaviour & $224 / 383 / 115$ & $36 / 63 / 24$ & 17 & 0.609 \\
\hline (never/former/current) & (31.0\%/53.0\%/15.9\%) & $(29.3 \% / 51.2 \% / 19.5 \%)$ & & \\
\hline Prior cardiovascular disease & $126(17.5 \%)$ & $25(24.3 \%)$ & 37 & 0.094 \\
\hline Current depression & 28 (3.9\%) & $11(8.3 \%)$ & 8 & 0.024 \\
\hline Carotid IMT (mm) & $0.85 \pm 0.15$ & $0.87 \pm 0.16$ & 37 & 0.346 \\
\hline Carotid IAD (mm) & $7.81 \pm 0.84$ & $8.01 \pm 0.97$ & 37 & 0.047 \\
\hline Carotid LD (mm) & $6.11 \pm 0.73$ & $6.28 \pm 0.84$ & 37 & 0.051 \\
\hline CWSpulsatile (kPa) & 24 [19-29] & 25 [21-29] & 53 & 0.058 \\
\hline CWSmean (kPa) & $48 \pm 11$ & $50 \pm 11$ & 37 & 0.038 \\
\hline Memory & $0.05 \pm 0.93$ & $-0.20 \pm 1.04$ & 9 & 0.014 \\
\hline Processing speed & $0.03 \pm 0.78$ & $-0.07 \pm 0.80$ & 15 & 0.209 \\
\hline Executive function \& attention & $0.03 \pm 0.81$ & $-0.09 \pm 0.88$ & 18 & 0.146 \\
\hline
\end{tabular}

Data are presented as mean \pm SD, median [IQR], or $n(\%) .{ }^{*}$ Number of missings for a specific variable. ${ }^{\dagger} \mathrm{P}$-value for difference between individuals included and those excluded from the main analyses. ${ }^{\ddagger}$ Obtained from office blood pressure measurements. ${ }^{\S}$ Based on the results of an oral glucose tolerance test and prescribed medication, as detailed elsewhere. ${ }^{10}$ Abbreviations: SBP = systolic blood pressure; DBP = diastolic blood pressure. 
Table S4.2 Associations between circumferential wall stress and memory function, stratified according to diabetes status

\begin{tabular}{lcc}
\hline Memory & T2DM- & T2DM+ \\
\hline CWS pulsatile & & \\
Model 1 & & 0.064 \\
& -0.047 & $(-0.069$ to 0.198$)$ \\
Model 2 & $(-0.124$ to 0.030$)$ & 0.089 \\
Model 3 & -0.043 & $(-0.042$ to 0.219$)$ \\
& $(-0.123$ to 0.038$)$ & 0.092 \\
CWS ${ }_{\text {mean }}$ & -0.045 & $(-0.040$ to 0.224$)$ \\
Model 1 & $(-0.126$ to 0.036$)$ & \\
Model 2 & & 0.097 \\
Model 3 & -0.059 & $(-0.036$ to 0.230$)$ \\
& $(-0.137$ to 0.018$)$ & 0.118 \\
& -0.059 & $(-0.012 ; 0.249)$ \\
& $(-0.140$ to 0.021$)$ & 0.120 \\
& -0.060 & $(-0.011$ to 0.251$)$ \\
\hline
\end{tabular}

$\mathrm{N}=722$ (197 with type 2 diabetes). Data are presented as standardised regression coefficient (95\% confidence interval), which reflect the change in cognitive performance per standard deviation increase in pulsatile (CWS pulsatie $_{\text {) }}$ or mean (CWS mean $_{\text {) circumferential wall stress. CWS }}$ was transformed with the natural logarithm prior to analysis. Model 1: adjusted for age, sex, and educational level; Model 2: additional adjustment for body mass index, total/high density lipoprotein-cholesterol ratio, triglycerides, use of lipid-modifying medication, hypertension, presence of type 2 diabetes, estimated glomerular filtration rate, smoking behaviour, alcohol consumption, and history of cardiovascular disease(s); Model 3: additional adjustment for the presence of a current depression. Abbreviations: T2DM = type 2 diabetes mellitus; CWS = circumferential wall stress.

$\mathrm{N}=722$. Data are presented as standardised regression coefficient (95\% confidence interval), which reflect the change in cognitive performance per standard deviation increase in intima-media thickness, interadventitial diameter, lumen diameter, pulse pressure, mean arterial pressure, or lumen-to-wall ratio. Model 1: adjusted for age, sex, and educational level; Model 2: additional adjustment for body mass index, total/high density lipoprotein-cholesterol ratio, triglycerides, use of lipid-modifying medication, hypertension, presence of type 2 diabetes, estimated glomerular filtration rate, smoking behaviour, alcohol consumption, and history of cardiovascular disease(s); Model 3: additional adjustment for the presence of a current depression. 
Table S4.3 Associations of individual components of circumferential wall stress and the lumen-to-wall ratio with cognitive performance

\begin{tabular}{|c|c|c|c|}
\hline Model & Memory & Processing speed & $\begin{array}{c}\text { Executive function } 8 \\
\text { attention }\end{array}$ \\
\hline \multicolumn{4}{|c|}{ Intima-media thickness } \\
\hline \multirow[t]{2}{*}{ Model 1} & -0.028 & 0.041 & 0.017 \\
\hline & $(-0.095$ to 0.039$)$ & $(-0.026$ to 0.108$)$ & $(-0.053$ to 0.086$)$ \\
\hline \multirow[t]{2}{*}{ Model 2} & -0.017 & 0.048 & 0.030 \\
\hline & $(-0.083$ to 0.049$)$ & $(-0.019$ to 0.114$)$ & $(-0.040$ to 0.099$)$ \\
\hline \multirow[t]{2}{*}{ Model 3} & -0.016 & 0.052 & 0.032 \\
\hline & $(-0.082$ to 0.051$)$ & $(-0.014$ to 0.119$)$ & $(-0.038$ to 0.101$)$ \\
\hline \multicolumn{4}{|c|}{ Interadventitial diameter } \\
\hline \multirow[t]{2}{*}{ Model 1} & -0.048 & 0.018 & -0.015 \\
\hline & $(-0.121$ to 0.024$)$ & $(-0.054$ to 0.091$)$ & $(-0.091$ to 0.060$)$ \\
\hline \multirow[t]{2}{*}{ Model 2} & -0.024 & 0.025 & -0.003 \\
\hline & $(-0.098$ to 0.050$)$ & (-0.050 to 0.099$)$ & $(-0.081$ to 0.075$)$ \\
\hline \multirow[t]{2}{*}{ Model 3} & -0.022 & 0.030 & -0.001 \\
\hline & $(-0.097$ to 0.052$)$ & $(-0.044$ to 0.105$)$ & $(-0.079$ to 0.077$)$ \\
\hline \multicolumn{4}{|c|}{ Lumen diameter } \\
\hline \multirow[t]{2}{*}{ Model 1} & -0.041 & 0.001 & -0.025 \\
\hline & $(-0.112$ to 0.031$)$ & $(-0.070$ to 0.072$)$ & $(-0.099$ to 0.049$)$ \\
\hline \multirow[t]{2}{*}{ Model 2} & -0.018 & 0.004 & -0.018 \\
\hline & $(-0.091$ to 0.055$)$ & $(-0.070$ to 0.077$)$ & $(-0.095$ to 0.058$)$ \\
\hline \multirow[t]{2}{*}{ Model 3} & -0.017 & 0.007 & -0.017 \\
\hline & $(-0.090$ to 0.056$)$ & $(-0.066$ to 0.081$)$ & $(-0.094$ to 0.060$)$ \\
\hline \multicolumn{4}{|c|}{ Pulse pressure (carotid artery) } \\
\hline \multirow[t]{2}{*}{ Model 1} & -0.009 & 0.011 & -0.026 \\
\hline & $(-0.077$ to 0.059$)$ & $(-0.057$ to 0.079$)$ & $(-0.097$ to 0.044$)$ \\
\hline \multirow[t]{2}{*}{ Model 2} & 0.006 & 0.017 & 0.001 \\
\hline & $(-0.065$ to 0.076$)$ & $(-0.054$ to 0.088$)$ & $(-0.074$ to 0.075$)$ \\
\hline \multirow[t]{2}{*}{ Model 3} & 0.006 & 0.018 & 0.001 \\
\hline & $(-0.064$ to 0.076$)$ & $(-0.053$ to 0.089$)$ & $(-0.073$ to 0.075$)$ \\
\hline \multicolumn{4}{|c|}{ Mean arterial pressure (brachial artery) } \\
\hline \multirow[t]{2}{*}{ Model 1} & -0.011 & 0.029 & -0.019 \\
\hline & $(-0.075$ to 0.053$)$ & $(-0.034$ to 0.093$)$ & $(-0.085$ to 0.047$)$ \\
\hline \multirow[t]{2}{*}{ Model 2} & -0.027 & 0.009 & -0.015 \\
\hline & $(-0.096$ to 0.042$)$ & $(-0.061$ to 0.078$)$ & $(-0.087$ to 0.058$)$ \\
\hline \multirow[t]{2}{*}{ Model 3} & -0.026 & 0.012 & -0.014 \\
\hline & $(-0.095$ to 0.043$)$ & $(-0.057$ to 0.081$)$ & $(-0.087$ to 0.059$)$ \\
\hline \multicolumn{4}{|c|}{ Lumen-to-wall ratio } \\
\hline \multirow[t]{2}{*}{ Model 1} & 0.009 & -0.031 & -0.021 \\
\hline & $(-0.056$ to 0.074$)$ & $(-0.096$ to 0.034$)$ & $(-0.088$ to 0.047$)$ \\
\hline \multirow[t]{2}{*}{ Model 2} & 0.012 & -0.036 & -0.028 \\
\hline & $(-0.052$ to 0.076$)$ & (-0.101 to 0.029$)$ & $(-0.096$ to 0.040$)$ \\
\hline \multirow[t]{2}{*}{ Model 3} & 0.011 & -0.038 & -0.029 \\
\hline & $(-0.053$ to 0.076$)$ & $(-0.103$ to 0.026$)$ & $(-0.097$ to 0.039$)$ \\
\hline
\end{tabular}


Chapter 4

Table S4.4 Associations between circumferential wall stress and cognitive performance

\begin{tabular}{lccc}
\hline Memory & Processing speed & $\begin{array}{c}\text { Executive function and } \\
\text { attention }\end{array}$ \\
\hline $\begin{array}{l}\text { CWS } \text { pulsatile } \\
\text { Model 1 }\end{array}$ & -0.007 & -0.020 & -0.018 \\
& $(-0.069$ to 0.055$)$ & $(-0.081$ to 0.041$)$ & $(-0.083$ to 0.046$)$ \\
CWS & & -0.009 \\
Model 1 & -0.000 & -0.004 & $(-0.073$ to 0.055$)$ \\
& $(-0.062$ to 0.061$)$ & $(-0.065$ to 0.057$)$ & \\
\hline
\end{tabular}

$\mathrm{N}=788$. Data are presented as standardised regression coefficient ( $95 \%$ confidence interval). Abbreviations: CWS = circumferential wall stress. Model 1: adjusted for age, sex, and educational level. No additional covariates were incorporated in the model to prevent exclusion of individuals because of missing data. 
Circumferential wall stress and cognition 


\section{Chapter 5}

\section{GLUCOSE REGULATION, COGNITION, AND BRAIN MRI IN TYPE 2 DIABETES: A SYSTEMATIC REVIEW}

Stefan L.C. Geijselaers, Simone J.S. Sep, Coen D.A. Stehouwer, Geert Jan Biessels 


\title{
Chapter 5
}

\begin{abstract}
Type 2 diabetes mellitus is associated with cognitive dysfunction and structural brain changes. Abnormalities in glucose regulation are involved in several complications related to type 2 diabetes, but their role in these cerebral complications is unclear. We systematically reviewed studies on the association between glucose regulation (glycaemia, hypoglycaemic events, insulin concentration, insulin resistance, and glucose-lowering treatment) and cognitive function and brain abnormalities on MRI in people with type 2 diabetes. The 86 papers included showed that glycaemia, particularly high $\mathrm{HbA1c}$ concentration and glucose variability, are negatively associated with cognitive function in people with type 2 diabetes without dementia. However, the strength of this association is weak, and HbA1c generally accounted for less than $10 \%$ of the variance in cognition. Importantly, few studies have measured long-term cerebral outcomes, such as dementia and structural brain changes on MRI, and the effect of glucose-lowering treatment on these outcomes. More randomised controlled trials are needed to establish the effect of glucoselowering treatment on long-term cognitive function in people with type 2 diabetes.
\end{abstract}




\section{Introduction}

Cognitive impairment and dementia are increasingly recognised as clinically important complications of type 2 diabetes. People with type 2 diabetes have a 1.5-fold increased risk of dementia, are more prone to subtle cognitive impairments, and have accelerated cognitive decline compared with people without diabetes. ${ }^{1,2}$ Moreover, the presence of ageing-related structural brain changes on MRI, particularly atrophy and markers of small vessel disease, are increased in people with diabetes. ${ }^{3,4}$ However, the factors that contribute to the onset and progression of cerebral complications in people with type 2 diabetes are unclear. In an ageing population with increasing prevalence of diabetes, the need to identify these factors is urgent. Abnormalities in glucose regulation have been implicated in several complications related to diabetes, including retinopathy ${ }^{5}$ and peripheral neuropathy. ${ }^{6}$ Glycaemic control can reduce the incidence and progression of these complications. ${ }^{5,6}$ For other complications, such as diabetes-associated large vessel disease, the role of glucose regulation is known to be less. ${ }^{7}$ Whether glucose regulation has a role in the cerebral complications of people with type 2 diabetes is unclear.

Glucose and insulin might directly affect brain structure and function. For example, high glucose concentrations can lead to the accumulation of aberrant metabolites, the depletion of important metabolic cofactors, and oxidative stress, giving rise to neuronal dysfunction and apoptosis. ${ }^{8}$ Chronic hyperglycaemia might lead to abnormalities in cerebral capillaries and altered cerebral blood flow. ${ }^{9,10}$ The effects of cerebral insulin are complex: it might improve cognitive performance by modulating synaptic plasticity, regulating the expression of several neurotransmitters, and increasing glucose availability in the brain. ${ }^{11}$ Insulin might also interfere with the metabolism of amyloid- $\beta$ and tau metabolism, which are key proteins in the pathogenesis of Alzheimer's disease. ${ }^{11}$ Hyperglycaemia and abnormalities in insulin signalling might, therefore, be involved in the cerebral complications of type 2 diabetes. However, alternative hypotheses need to be considered. A clear link exists between type 2 diabetes, vascular risk factors, and vascular disease. In the general population, these vascular factors contribute to age-related decline in cognitive function ${ }^{12,13}$ and might also affect cognition in people with type 2 diabetes, independent of glucose regulation. Although many studies have addressed the role of high glucose concentrations and insulin resistance in cognitive function in people with type 2 diabetes, their precise role is still debated. Resolution of this debate is important, because glucose dysregulation is amenable to treatment, and might be a target to prevent accelerated cognitive decline in people with type 2 diabetes. We aimed to systematically review the association of glucose regulation (ie, glycaemia, hypoglycaemic events, insulin concentration, insulin resistance, and glucoselowering treatment) with cognitive function and structural brain abnormalities on MRI 
in people with type 2 diabetes.

\section{Methods}

\section{Data sources and search strategy}

This systematic review was done according to the Preferred Reporting Items for Systematic reviews and Meta-Analysis) statement. ${ }^{14}$ Study selection, data extraction, and risk of bias assessment were done by SLCG on the basis of formats used in other systematic reviews. ${ }^{3,9,13,15}$ In cases of doubt, SJSS, CDAS, and GJB were consulted.

Medline (PubMed) and Embase were searched for relevant papers with a combination of free search terms and subject headings referring to type 2 diabetes, cognition, and brain MRI abnormalities (see Supplement for detailed description of search methodology). The aim was to include all papers with original data for the association between glucose regulation and cognitive function, brain abnormalities on $\mathrm{MRI}$ in people with type 2 diabetes, or both. Data for between-group comparisons of people with or without diabetes were not considered. Only studies of human being that were written in English and published after 1990 were included because MRI was not available before 1990 . The final search was done on June 17, 2014. Reference lists of included papers were checked for additional eligible studies. Cross-checks with literature databases collected by GJB from 1992 onwards identified no missed citations.

\section{Study selection}

Study selection was done in two stages. First, studies were selected on the basis of the title and abstract that included (1) a subgroup of at least 40 participants with type 2 diabetes or diabetes without further specification, and (2) reported an outcome measure of cognitive function, brain abnormalities on MRI, or both.

In the second stage, the full text of eligible papers was assessed for two criteria. First, the paper had to be about original research that reported the association between measures of glucose regulation and cognitive function or brain abnormalities on MRI in people with type 2 diabetes. If a study did not distinguish between 1 or type 2 diabetes, it was included only if the mean age of the study population was older than 50 years, under the assumption that most participants would have type 2 diabetes, as suggested by epidemiological data. ${ }^{16}$ Second, studies had to have a sample size of at least 40 in the analysis of interest. This was based on the knowledge that diabetes-associated cognitive decrements are rather small (with effect sizes of 0.3-0.5 SD units in cross-sectional studies). ${ }^{15}$ The same is true for diabetes-associated structural brain changes on MRI. ${ }^{4,17}$ Therefore, studies with few participants might have insufficient statistical power to 
detect associations.

In this systematic review, glucose regulation was defined as measures of glycaemia (ie, $\mathrm{HbA1c}$, fasting blood glucose concentrations, non-fasting blood glucose concentrations, and glucose variability), hypoglycaemic events, insulin concentration and insulin resistance (in practice this was limited to fasting insulin concentration or the assessment of insulin resistance by HOMA-IR [homeostasis model assessment of insulin resistance]), and glucose-lowering treatment. Because diabetes-associated cognitive dysfunction ranges from subtle cognitive impairment to overt dementia, ${ }^{1}$ and can be assessed in many ways, any objective method to assess or classify cognitive function was included in this review. Studies that used subjective, self-reported measures of cognitive function were not included because their correlation with objective measures is weak. ${ }^{18}$ Brain abnormalities on MRI were defined as structural abnormalities associated with diabetes (ie, small vessel disease and brain atrophy). To avoid heterogeneity in the terminology for brain abnormalities on MRI we used the standards for reporting vascular changes on neuroimaging (STRIVE). ${ }^{19}$

Several papers on the same study population were included only if different determinants or outcome measures were assessed in separate papers. For papers reporting on the same determinant and outcome measure, we included only the publication with the most detailed information. Studies were excluded if population data were not appropriately adjusted for differences in age, sex, or education level (when cognitive function was an outcome measure) between groups. Studies of induced acute hypoglycaemia or hyperglycaemia were also excluded.

\section{Data extraction and synthesis}

Information was taken from each study about the following items: general demographic and diabetes-specific characteristics (eg, diabetes duration and diabetes-related complications); measures of glucose regulation; measures of cognitive functioning and brain MRI; covariates included in the analyses of interest (eg, vascular risk factors and comorbid conditions).

We present results derived from statistical analyses that controlled for demographic characteristics and vascular risk factors, when available. Data for demographic characteristics were age, sex, education level, and socioeconomic status. Vascular risk factors included measures of obesity or central obesity, blood pressure, lipids, smoking, physical activity, and history of cardiovascular disease. We only report effect measures (ie, standardised regression coefficient $[\beta]$, odds ratio $[O R]$, and hazard ratio $[H R]$ ) adjusted for age, sex, and education level (for cognitive outcome measures) in tables 
because these are well-established confounding factors in the assessment of cognitive function and structural MRI. For studies that did not adjust their statistical analyses for these confounding factors, we report only on the direction of the association. Because studies varied widely in methods, sample population, and outcome measures a metaanalysis was not done.

\section{Risk of bias assessment}

The risk of bias among studies reviewed was assessed with a slightly modified version (Supplement) of the Newcastle-Ottawa Quality Assessment Scale (NOS), in which a high score indicates a low risk of bias. ${ }^{20}$ Studies were classified as at low, moderate, or high risk of bias. For randomised controlled trials, we used the Cochrane Collaboration's tool $^{21}$ to assess the risk of bias. The risk of bias was not used as an exclusion criterion in the selection of studies to provide a complete overview of available data. However, to prevent bias in our own Review, the evidence was also considered with exclusion of studies at high risk of bias.

\section{Results}

\section{Overview}

The literature search yielded 6300 unique citations. The full text of 422 citations was examined and, of these, 86 articles on 70 unique studies met our inclusion criteria (Figure 5.1). Longitudinal analyses were done in 37 of the 86 included papers. Characteristics of the included papers are in the Supplement.

Cognitive function was an outcome measure in 78 articles..$^{22-99}$ Studies used various tests of cognitive function and screening methods to measure global cognitive function or performance in one or more specific cognitive domains (Supplement). Two papers reported cognitive impairment no dementia (CIND), based on a neuropsychological evaluation. ${ }^{50,97} 15$ papers focused on dementia (of these, two combined dementia with CIND). ${ }^{83,97}$ A diagnosis of dementia was based on medical records (using the International Classification of Diseases $22,32,37,47,81,89,95$ or criteria of the Diagnostic and Statistical Manual of mental disorders ${ }^{85}$ ) or a diagnostic procedure that consisted of cognitive screening followed by an extensive neuropsychological and neurological examination in those who screened positive. ${ }^{50,57,83,86,91,97}$ One paper did not specify how the presence of dementia was determined. ${ }^{79}$ Brain MRI abnormalities were assessed in 16 articles (Supplement). ${ }^{25,30,33,34,58,62,63,96,100-107}$ The findings of these studies are described in Panel 5.1 . 
Type 2 diabetes, cognition, and brain MRI

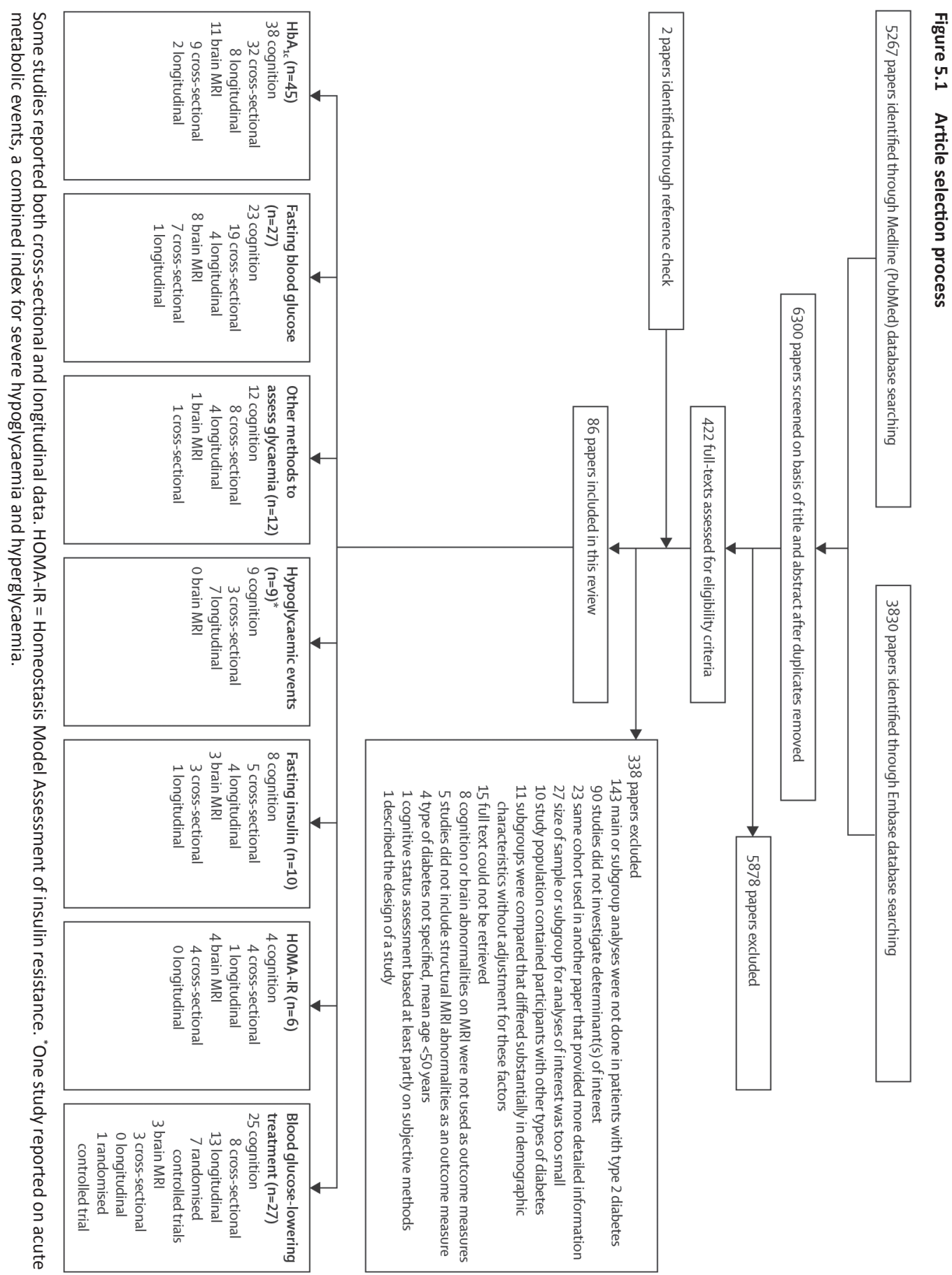




\section{Panel 5.1 - Brain abnormalities on MRI}

\section{Brain atrophy}

Brain atrophy is defined as a low brain volume that is not related to a specific macroscopic focal injury (eg, trauma or infarction). ${ }^{19}$ Atrophy can be global or focal (affecting a specific brain region or tissue class), affect the brain symmetrically or asymmetrically, and might not necessarily reflect neuronal cell loss. ${ }^{19}$

A total of 11 papers $25,30,33,58,62,96,101,103-105,107$ explored the association between measures of glucose regulation and brain atrophy in people with type 2 diabetes. Of cross-sectional studies, $\mathrm{HbA} 1 \mathrm{c}$ (five $\mathrm{f}^{3062,103,105,107}$ ), fasting insulin (one ${ }^{58}$ ), and HOMA-IR (homeostasis model assessment of insulin resistance; three ${ }^{25,58,104}$ ) were not associated with the presence of brain atrophy. $\mathrm{HbA1c}$ concentrations were not associated with changes in brain volume after four years. ${ }^{101}$ Two $^{104,107}$ (50\%) of four ${ }^{30,58,104,107}$ crosssectional studies reported that high fasting blood glucose concentrations were inversely related to brain volume, possibly reflecting osmotic effects of short-term hyperglycaemia. Glucose variability was correlated with gray matter volumes. ${ }^{96}$ Insulin use was not associated with the presence of brain atrophy in one observational study. ${ }^{104} \mathrm{~A}$ randomised trial that assessed the effects of intensified versus regular glucose-lowering treatment showed that intensified treatment was associated with a reduced decline in total brain volume. ${ }^{33}$

\section{White matter hyperintensities}

Hyperintensities are regions of high signal intensity on T2-weighted MRI. ${ }^{19}$ White matter hyperintensities appear as bright areas on MRI. They most often occur bilaterally, are often symmetrical, and can vary in size. ${ }^{19}$ White matter hyperintensities are strongly associated with vascular disease and vascular risk factors, but their exact pathogenesis is not well understood and might be multifactorial. ${ }^{19}$

Ten papers ${ }^{33,34,58,62,63,100-102,104,107}$ explored the association between measures of glucose regulation and white matter hyperintensities in people with type 
2 diabetes. HbA1c concentations were not associated with the presence (five studies ${ }^{62,63,100,102,107}$ ) or progression (two studies ${ }^{34,101}$ ) of white matter hyperintensities. Two ${ }^{102,107}$ (33\%) of six ${ }^{58,63,97,102,104,107}$ cross-sectional studies showed a significant, but weak, association between high fasting blood glucose concentration and white matter hyperintensities; however, the associations reported for these two studies were in opposite directions and one $\mathrm{e}^{102}$ was judged to be at high risk of bias. Fasting blood glucose concentrations were not associated with the progression of white matter hyperintensities in one study. ${ }^{34}$ Of studies that assessed fasting insulin concentrations ${ }^{58,63,102}$ and HOMA-IR $\mathrm{R}^{58,102,104}$ two $^{58,102}$ reported associations with the presence of white matter hyperintensities. Fasting insulin concentrations were not associated with the progression of white matter hyperintensities. ${ }^{34}$ Neither the use of insulin ${ }^{104}$ nor pioglitazone ${ }^{102}$ was associated with the presence of white matter hyperintensities. However, compared with regular glucose-lowering treatment, intensified glucoselowering treatment was associated with a higher volume of white matter hyperintensities at 40 months of follow-up in a randomised controlled trial. $^{33}$

\section{Cerebral infarcts}

Cerebral infarcts on MRI can be categorised as small subcortical infarcts or large-vessel infarcts. ${ }^{17,19}$ Four studies ${ }^{34,62,104,106}$ explored the association between measures of glucose regulation and cerebral infarcts in people with type 2 diabetes. Three cross-sectional studies showed that HbA1C concentration (two ${ }^{62,106}$ ), fasting blood glucose concentration (one ${ }^{104}$ ), and HOMA-IR (one $\mathrm{C}^{104}$ ) were not associated with the presence of cerebral infarcts. In a longitudinal study, the progression of recent small subcortical infarcts was not associated with $\mathrm{HbA1c}$, fasting blood glucose, or fasting insulin concentrations. ${ }^{34}$ Insulin use was associated with the presence of small subcortical cerebral infarcts in one ${ }^{106}$ of two studies. ${ }^{104,106}$ 
Risk of bias in the included articles is shown in detail in the Supplement and the NOS is also shown in Tables 5.1-5.3. In general, the directionality of the associations did not relate to the risk of bias. Overall, the risk of bias was moderate in cross-sectional studies (mean NOS 3 out of a maximum of 5) and low in longitudinal studies (mean NOS 7 out of a maximum of 8). Frequently, effect measures reported were unadjusted for demographic characteristics in the target population or the target population was not representative of an average population with diabetes. Most of the randomised controlled trials included had a low or unclear risk of bias.

\section{HbA1c concentration}

32 cross-sectional and eight longitudinal studies related $\mathrm{HbA} 1 \mathrm{c}$ to cognition (two studies reported on both cross-sectional and longitudinal data ${ }^{65,92}$ ). HbA1c concentration was most often assessed with a single blood sample. Two studies used medical records to obtain single $\mathrm{e}^{55}$ or multiple ${ }^{99} \mathrm{HbA1c}$ values. Four studies did not specify how $\mathrm{HbA1c}$ concentration was assessed. $24,30,56,90$ The mean $\mathrm{HbA} 1 \mathrm{c}$ concentration ranged from $6.3 \%$ to $9.5 \%$ (45 $\mathrm{mmol} / \mathrm{mol}$ to $80 \mathrm{mmol} / \mathrm{mol}$ ); 23 studies had a mean HbA1c concentration higher than $7 \%$ (53 mmol/mol). With one exception, ${ }^{42}$ studies were done in populations without cognitive impairment or dementia.

$15(47 \%)$ of 32 cross-sectional studies reported a significant, but weak, negative association between $\mathrm{HbA} 1 \mathrm{C}$ concentrations and cognitive function (Table 5.1). ${ }^{24,34,35,41,42 \text {, }}$ $44,48,52,59,62,64,67,73,90,99$ Executive function and attention were investigated in 19 studies and five $(26 \%)$ of these found a negative association with $\mathrm{HbA} 1 \mathrm{c}$ concentration. ${ }^{41,42,73,90,99}$ For memory, four (22\%) of 18 studies, ${ }^{34,44,48,52}$ and for information processing speed, six (38\%) of 16 studies, ${ }^{48,52,62,64,67,73}$ reported a negative association. Studies reported various effect measures, including correlation and regression coefficients. Regardless of adjustments, correlation coefficients that were significant ranged from -0.46 to -0.07 and significant standardised regression coefficients ranged from -0.25 to -0.16 , indicating that the explained variance in cognition by $\mathrm{HbA} 1 \mathrm{c}$ concentrations was at most 10 to $15 \%$. Only standardised regression coefficients that were adjusted for age, sex, and educational level are presented in Table 5.1.

In general, the proportion of cross-sectional studies reporting a significant association between $\mathrm{HbA} 1 \mathrm{c}$ and cognitive performance did not differ when the risk of bias was considered. When we excluded studies at high risk of bias, ${ }^{24,30,44,55,56,59,72,73,78,90}$ ten (45\%) of 22 studies showed a significant association. When we focused solely on studies judged to be at low risk of bias, ${ }^{26,34,35,40,48,52,53,62,65,74,77,80,92,99}$ six (43\%) of 14 studies showed a significant negative association of $\mathrm{HbA1c}$ concentration with cognitive function. 
Results were similar in studies that did, 12 (38\%) of 32, and did not, 20 (62\%) of 32, adequately adjust for demographic characteristics. Studies that reported a significant association did not differ from those that did not in terms of participants' mean HbA1c concentration, diabetes duration, glucose-lowering treatment, and the presence of diabetes-related complications or comorbidities.

Ten cross-sectional studies (31\%) took into account the possible modulating effects of vascular risk factors. ${ }^{26,34,35,40,48,53,74,80,92,99}$ Of these, six studies included blood pressure s4,40,99 $^{34}$ or the presence of hypertension ${ }^{26,53,74}$ as a covariate in their analysis. Vascular risk factors were often included in the basic statistical model. Overall, the results of studies that did and did not control for vascular risk factors were comparable.

One study investigated the relation between trajectories of $\mathrm{HbA} 1 \mathrm{c}$ concentration over time and cross-sectional cognitive performance. ${ }^{99}$ Six trajectories of $\mathrm{HbA} 1 \mathrm{c}$ were identified according to $\mathrm{HbA} 1 \mathrm{c}$ concentration (higher or lower) at entry into a diabetes registry and trend over time (stable, decreasing, or increasing). Starting HbA1c concentration was highest in patients whose $\mathrm{HbA1c}$ decreased over time and was lowest in those with stable $\mathrm{HbA} 1 \mathrm{c}$ over time. Patients with trajectories of decreasing $\mathrm{HbA1c}$ concentration had the worst cognitive performance, whereas patients with stable $\mathrm{HbA1c}$ concentration had the highest test scores.

Eight longitudinal studies investigated the relation between $\mathrm{HbA1c}$ concentration and cognitive function, with follow-up ranging from 6 months to 14 years. ${ }^{27,38,39,65,81,87,89,92}$ The risk of dementia was investigated in three studies: $: 1,89,92$ only one reported that high concentrations of $\mathrm{HbA1c}$ (from $10 \%$ [86 mmol$/ \mathrm{mol}$ ] to $12 \%$ [108 mmol/mol]) were associated with an increased risk of developing dementia, ${ }^{89}$ whereas low concentrations of $\mathrm{HbA} 1 \mathrm{c}$ were not. Cognitive decline was investigated in six studies, with mixed results: three studies, ${ }^{27,38,92}$ of which two had adjusted analyses for demographic characteristics, ${ }^{38,92}$ found no relation between $\mathrm{HbA} 1 \mathrm{c}$ concentration and cognitive decline or acceleration. Another study involving only patients aged 85 years at baseline showed that high $\mathrm{HbA1c}$ concentrations were associated with a slower decline in memory function; ${ }^{65}$ however, the other two studies reported a negative association. ${ }^{39,87}$ One of these studies ${ }^{87}$ related mean $\mathrm{HbA1c}$ concentration during follow-up to cognitive changes over 10 years. In participants with a previous diagnosis of diabetes, high $\mathrm{HbA1c}$ concentrations were associated with a faster decline in memory function and fluid intelligence than were low HbA1c concentrations. In participants with newly diagnosed diabetes, high HbA1c concentrations were only associated with fast decline in fluid intelligence. In the other study, high $\mathrm{HbA1c}$ concentration at baseline was associated with an increased risk of cognitive impairment within 6 months after coronary bypass surgery, although the risk 


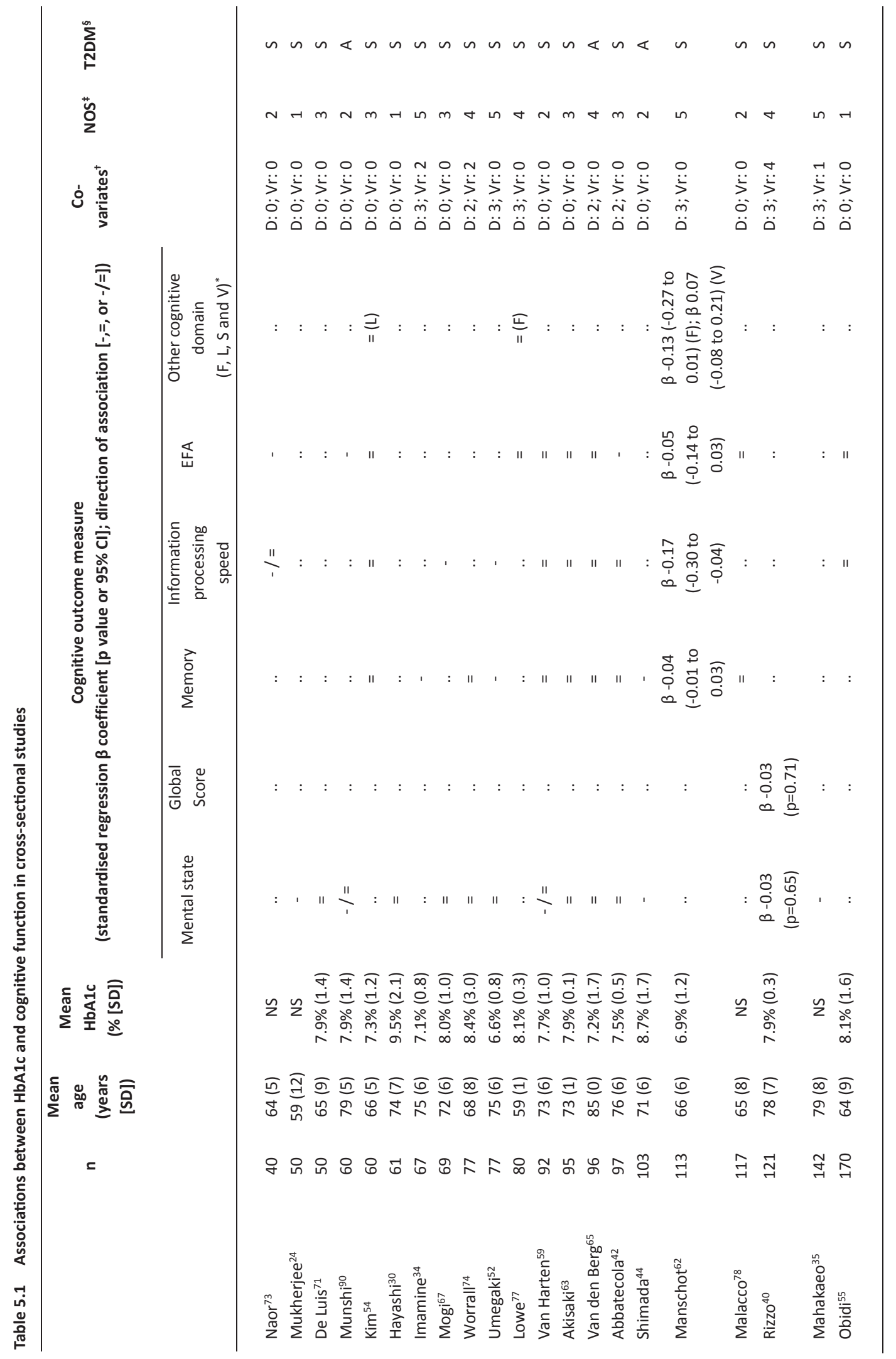




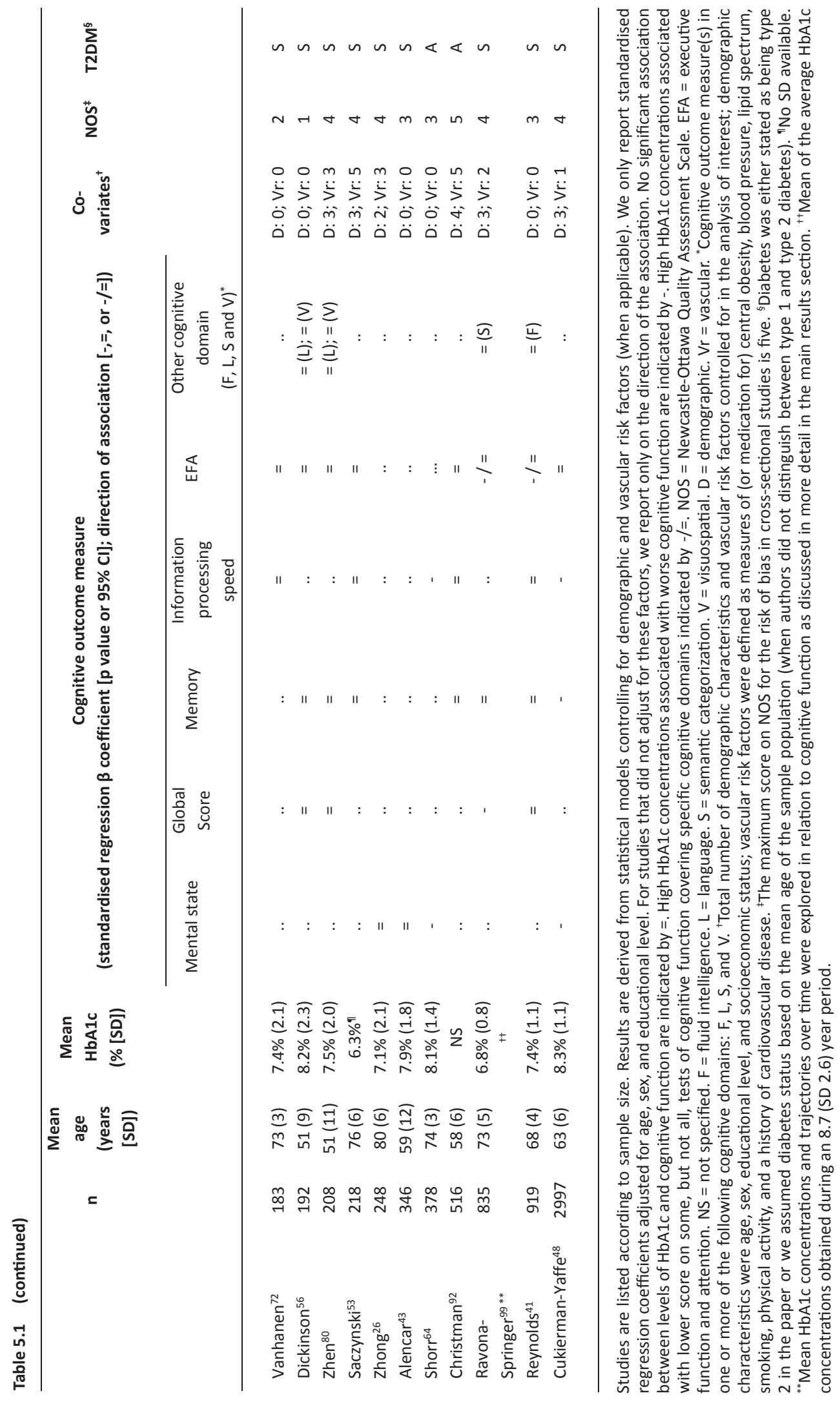


of bias was high in this study. ${ }^{39}$

\section{Fasting blood glucose concentrations}

The association between fasting blood glucose and cognition was investigated in 19 crosssectional and four longitudinal studies. Fasting blood glucose concentrations were usually based on a single blood sample, but in one study, ${ }^{55}$ fasting blood glucose concentrations were obtained through medical records. Another study ${ }^{30}$ did not specify how fasting blood glucose concentrations were collected. Across studies, mean fasting blood glucose concentrations ranged from 6.4 to $10.4 \mathrm{mmol} / \mathrm{L}$. Most cross-sectional studies did not report whether fasting blood sampling and cognitive assessment took place on the same day. Most cross-sectional studies (15 [79\%] of 19) found no relation between fasting blood glucose and cognition (Table 5.2). The four studies that did report significant associations showed a remarkably variable result, with negative ${ }^{23}(5 \%)$, positive ${ }^{55}(5 \%)$, or bell-shaped ${ }^{64,66}(10 \%)$ associations. The risk of bias did not correlate with study results. When studies with a high risk of bias ${ }^{30,44,55,72,75}$ were excluded, the percentage of studies that showed no relation between fasting blood glucose concentrations and cognitive performance remained unchanged (11 [79\%] of 14). In studies with a low risk of bias, ${ }^{23,26,40,48,51,58,64,76,80}$ seven (78\%) of nine showed no correlation. No differences were found in the results of studies that did $(47 \%)$ or did not $(53 \%)$ adjust for demographic characteristics.

Four longitudinal studies investigated the relation between fasting blood glucose concentrations and cognition, with follow-up ranging from 7 days (postoperative) to 14.7 years. ${ }^{34,38,39,97}$ Two studies reported a significant association, albeit in opposite directions..$^{34,97}$ One reported that high fasting glucose concentrations were associated with a decline in processing speed (standardised regression coefficient $-0.259, p<0.05$ ), ${ }^{34}$ whereas the other showed that high fasting blood glucose concentrations were associated with a decreased risk of incident cognitive impairment (OR 0.80, 95\% Cl 0.69 to 0.94$).{ }^{97}$

\section{Other measures of glycaemia}

Eight cross-sectional and four longitudinal studies reported the association between on one or more other measures of glycaemia (eg, glucose variability, non-fasting glucose concentrations, or glycaemic control) and cognitive function.

Three cross-sectional studies used continuous glucose monitoring systems to assess fluctuations in glucose levels. ${ }^{26,40,96}$ Glucose variability was negatively associated with cognitive function in all three studies, even after adjustment for glycaemic control ${ }^{26,40,96}$ and vascular risk factors. ${ }^{26,40}$ Excursions in fasting and postprandial blood glucose 
concentrations (based on frequent capillary and venous blood sampling) were negatively associated with cognitive performance in another study, even after adjustment for demographic characteristics, depression, and vascular risk factors. ${ }^{60}$ Longitudinal changes in glucose concentrations were correlated with cognitive performance in two other studies, ${ }^{61,78}$ although the risk of bias was high in these studies.

The association of non-fasting blood glucose concentrations with cognitive function was investigated in four cross-sectional studies and one longitudinal study. In one study, ${ }^{72}$ high $2 \mathrm{~h}$ postload glucose concentrations (after a $75 \mathrm{~g}$ oral glucose tolerance test) correlated with worse information processing speed, but there was no association with executive function and attention. Another found no correlation between test scores and capillary glucose concentrations before testing. ${ }^{73}$ Both studies were, however, at high risk of bias. ${ }^{72,73}$ Two other studies ${ }^{26,40}$ related postprandial glucose concentrations to cognitive functions. One reported a significant association between high postprandial glucose concentrations and low cognitive function. ${ }^{40}$ In a longitudinal study ${ }^{39}$ judged to have a high risk of bias, peak perioperative glucose concentrations did not differ between patients who did and did not develop impaired cognitive function within 7 days of coronary bypass surgery.

One study ${ }^{49}$ used a combined index of glycaemic control based on fasting and postprandial blood glucose concentrations, and showed no correlation between glycaemic control and cognitive variables. Another study ${ }^{86}$ computed a 5-year mean blood glucose concentration based on measurements of fasting blood glucose, random blood glucose, and $\mathrm{HbA1c}$. Patients with the highest mean blood glucose concentrations had an increased risk of dementia. Changes in blood glucose concentrations during cognitive assessments were measured in one cross-sectional study. ${ }^{46}$ High rates of decline in peripheral blood glucose concentration were, in general, associated with better cognitive performance. ${ }^{46}$

\section{Hypoglycaemic events}

Three cross-sectional and six longitudinal studies related hypoglycaemic events to cognitive function and dementia (one study reported on both cross-sectional and longitudinal data $\left.{ }^{50}\right)$. Severe hypoglycaemia was generally defined as an episode when the patient needed external help. Studies used medical records $22,27,47,50,81$ or questionnaires ${ }^{28,50,67,84}$ (or both) to obtain data about hypoglycaemic events. The frequency of hypoglycaemic events was recorded in some studies. ${ }^{22,28,47,84}$ With one exception, ${ }^{67}$ all studies were judged to have a low risk of bias.

Two (67\%) of three cross-sectional studies showed a significant negative association between hypoglycaemic events and cognitive function after adjustment for demographic 


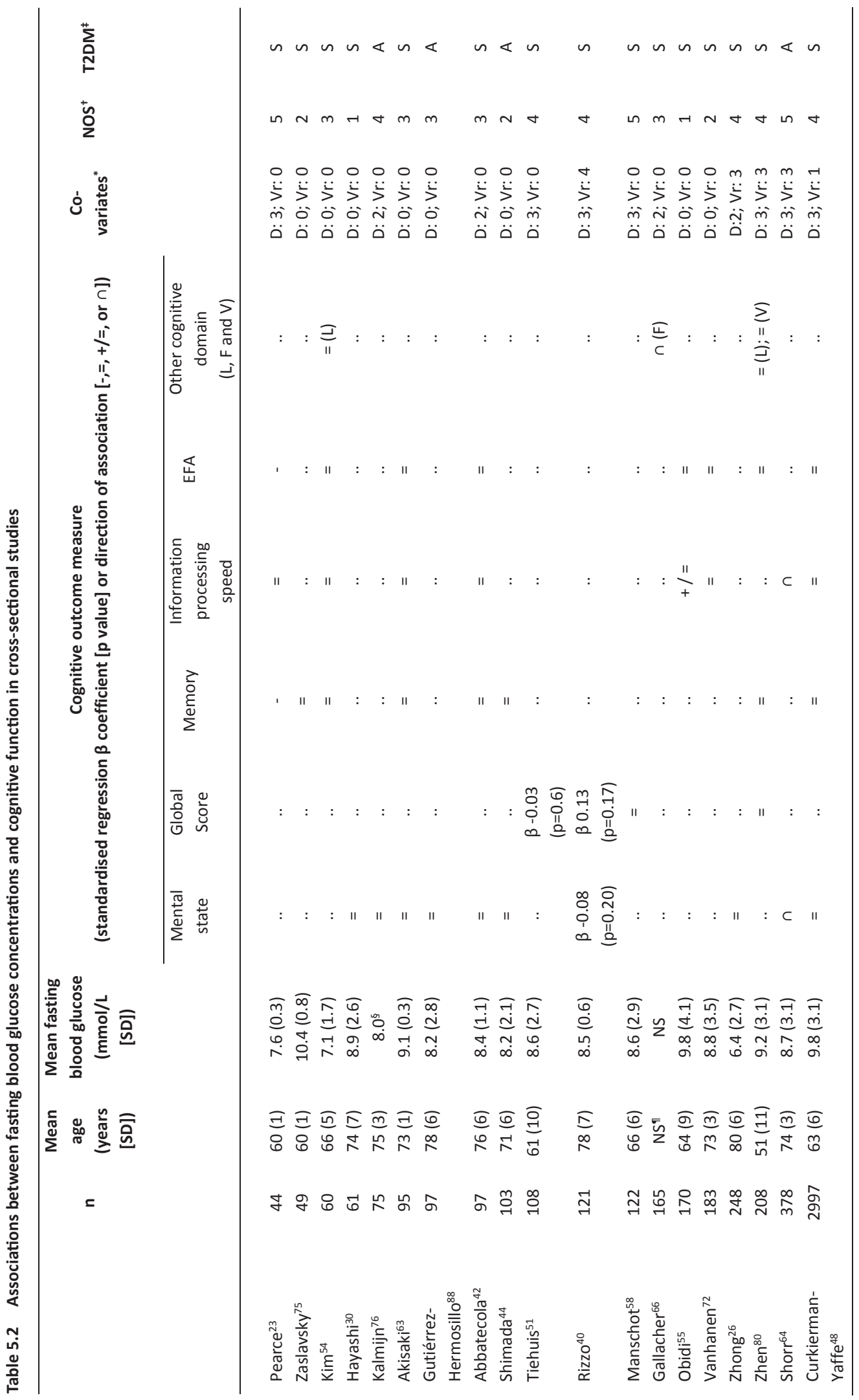


Studies are listed according to sample size. Results are derived from statistical models controlling for demographic and vascular risk factors (when applicable). We only report standardised regression coefficients adjusted for age, sex, and educational level. For studies that did not adjust for these factors, we report only on the direction of the association. No significant association between fasting blood glucose levels and cognitive function are indicated by $=$. High fasting blood glucose concentrations associated with worse cognitive function are indicated by -. Both low and high levels of fasting blood glucose concentrations associated with worse cognitive function are indicated by $\cap$. High fasting blood glucose concentrations associated with high scores on some, but not all, tests of cognitive function covering a specific cognitive domain are indicated by $+/=\mathrm{F}=$ fluid intelligence. $L=$ language. $V=$ visuospatial. $D=$ demographic. $V r=$ vascular. NOS = Newcastle-Ottawa Quality Assessment Scale. EFA = executive function and attention. NS = not specified. ${ }^{*}$ Total number of demographic characteristics and vascular risk factors controlled for in the analysis of interest; demographic characteristics were age, sex, educational level, and socioeconomic status; vascular risk factors were defined as measures of (or medication for) central obesity, blood pressure, lipid spectrum, smoking, physical activity, and a history of cardiovascular disease. 'Total maximum score on NOS for the risk of bias in cross-sectional studies is five. ${ }^{\ddagger}$ Diabetes was either stated as being type 2 in the paper or we assumed diabetes status based on the mean age of the sample population (when authors did not distinguish between type 1 and type 2 diabetes). ${ }^{5}$ No SD available. "Mean age not provided ages range from 55 to 69 years.

characteristics..$^{28,50}$ The other study, ${ }^{67}$ which had the smallest sample population $(n=69)$, did not adjust for demographic characteristics, and judged as having a moderate risk of bias, showed no correlation between hypoglycaemic events during the previous month and cognitive function. Only one cross-sectional study ${ }^{50}$ was focused on dementia and did not detect an association between cognitive function and hypoglycaemic events.

Two large longitudinal studies reported that a history of hypoglycaemic events at baseline was associated with an increased risk of development of dementia during a mean follow up of 3.8 years $^{22,47}$ (HR 1.45, 95\% Cl 1.07-1.95;22 and 1.44, 1.25-1.66; ${ }^{47}$ both were fully adjusted analyses). The risk of dementia increased as the number of hypoglycaemic events increased. ${ }^{22,47}$ One study reported that incident hypoglycaemic events were associated with an increased risk of development of dementia during 12 years of followup..$^{81}$ The relation between hypoglycaemic events and cognitive decline was investigated in three studies: two studies found no association between hypoglycaemia and cognitive decline over 1.5 years ${ }^{50}$ or 6 years. ${ }^{27}$ By contrast, the other study showed that both a history of hypoglycaemia and incident hypoglycaemia were associated with accelerated cognitive decline during 4 years of follow-up. ${ }^{84}$ One study related acute metabolic events (a combined index for severe hypoglycaemia or hyperglycaemia) to the risk of dementia. Patients who had an acute metabolic event were more likely to develop dementia during a mean follow-up of 6.6 years. ${ }^{89}$

\section{Fasting insulin and insulin resistance}

The relation between fasting insulin and cognitive function was investigated in five crosssectional and four longitudinal studies, and one study $\mathrm{y}^{42}$ included both cross-sectional and longitudinal data. Insulin resistance was assessed with HOMA-IR, and association with 
cognition was studied in four cross-sectional and one longitudinal study, and one study ${ }^{23}$ reported on both cross-sectional and longitudinal data. Fasting venous blood samples were used in all studies to determine insulin concentration and insulin resistance. Mean fasting insulin concentration ranged from 19 to $148 \mathrm{pmol} / \mathrm{L}$. HOMA-IR varied between 4.2 and 6.6. None of the studies focusing on insulin concentration or insulin resistance had a high risk of bias.

Two (40\%) of the five cross-sectional studies showed a significant, moderate, negative correlation between fasting insulin and different measures of cognitive function, such as the Mini Mental State Examination ${ }^{52}$ or memory function ${ }^{42}$ (Table 5.3). Measures of cognitive function in other domains, such as information processing speed ${ }^{42,52,63}$ and executive function and attention, ${ }^{42,63}$ showed no correlations with fasting insulin concentrations. One ${ }^{23}(25 \%)$ of four ${ }^{23,25,51,58}$ studies reported a negative association between HOMA-IR and cognitive function.

Three out of four longitudinal studies showed no significant association between fasting insulin concentration and changes in cognitive function ${ }^{38,42}$ or the risk of dementia. ${ }^{91}$ The other study showed that high fasting insulin concentrations were associated with a decline in executive function. ${ }^{93}$

One study on HOMA-IR and cognition, in participants who were overweight or obese and completed an 8-week energy-restricted diet, showed that a high HOMA-IR index was predictive of poorer cognitive performance at follow-up. ${ }^{23}$

\section{Glucose-lowering treatment}

Eight cross-sectional and 13 longitudinal observational studies explored the association between glucose-lowering treatment and cognitive function (of these, three reported on both cross-sectional and longitudinal data ${ }^{45,82,94}$ ). The risk of bias was high in four of these studies. ${ }^{43,45,68,70}$ Seven randomised controlled trials compared the effects of different treatment strategies on cognitive function.

Five $^{43,45,53,70,82}$ of eight ${ }^{43,45,53,68,70,82,85,94}$ cross-sectional observational studies did not report a significant difference in cognitive function with glucose-lowering treatments (Supplement). Two studies reported that people taking oral glucose-lowering treatment had better cognitive function ${ }^{68}$ or had a lower prevalence of dementia ${ }^{85}$ compared with people not taking glucose-lowering drugs. One of these studies was, however, judged as being at high risk of bias. ${ }^{68}$ In one study the use of metformin for more than 6 years, compared with non-use, was associated with a decreased risk of cognitive impairment. ${ }^{94}$ Seven longitudinal observational studies focused on the association between 


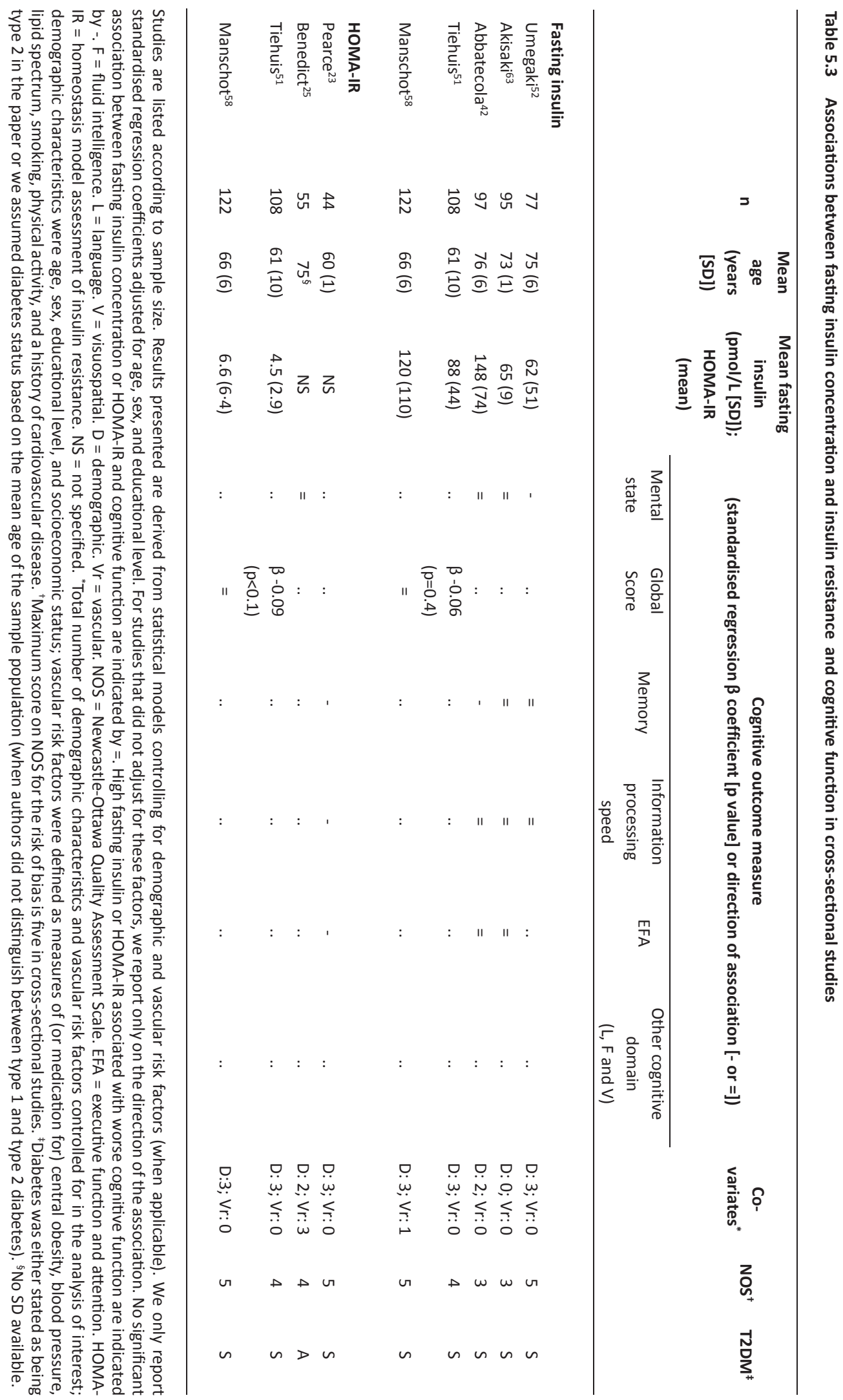




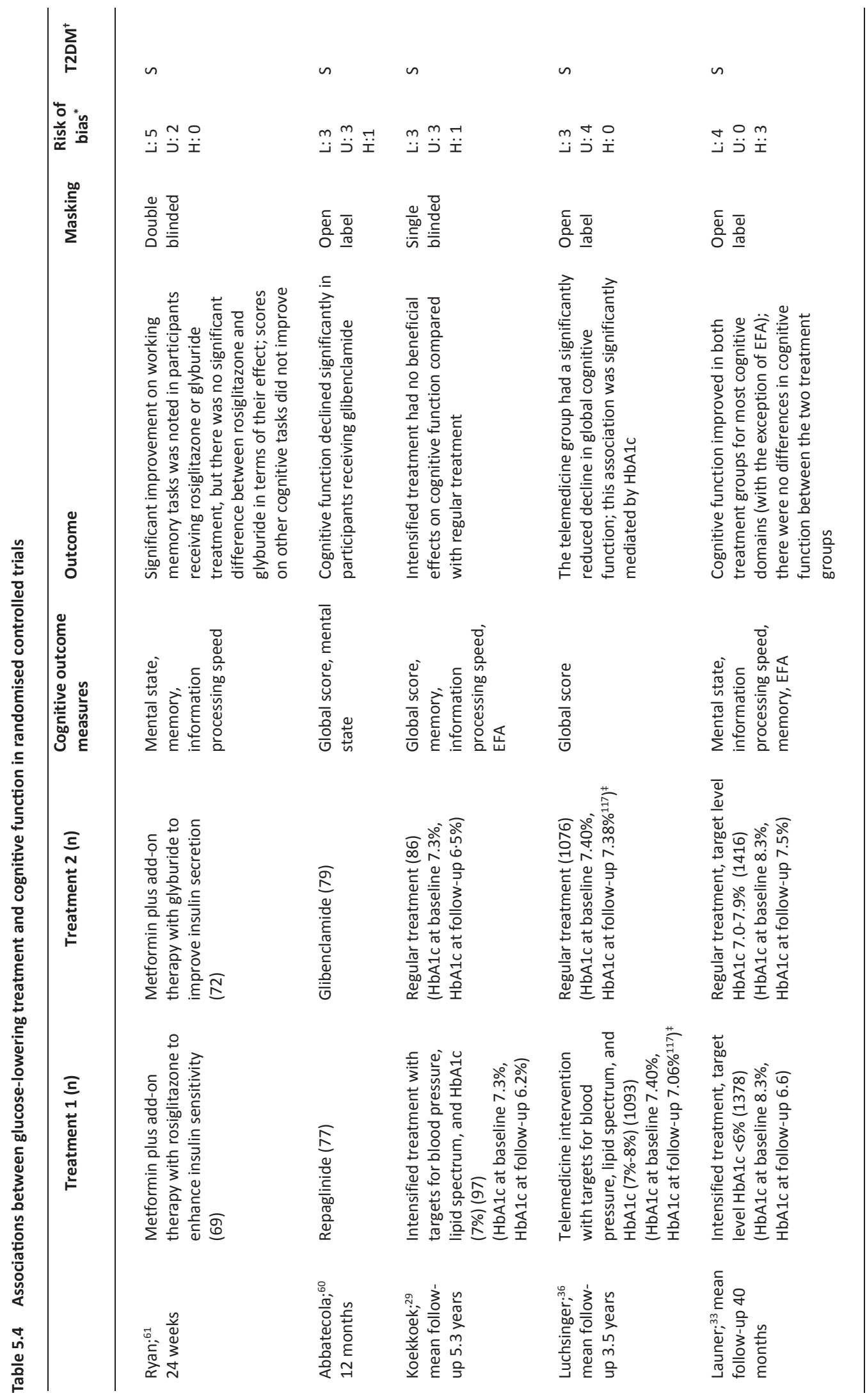




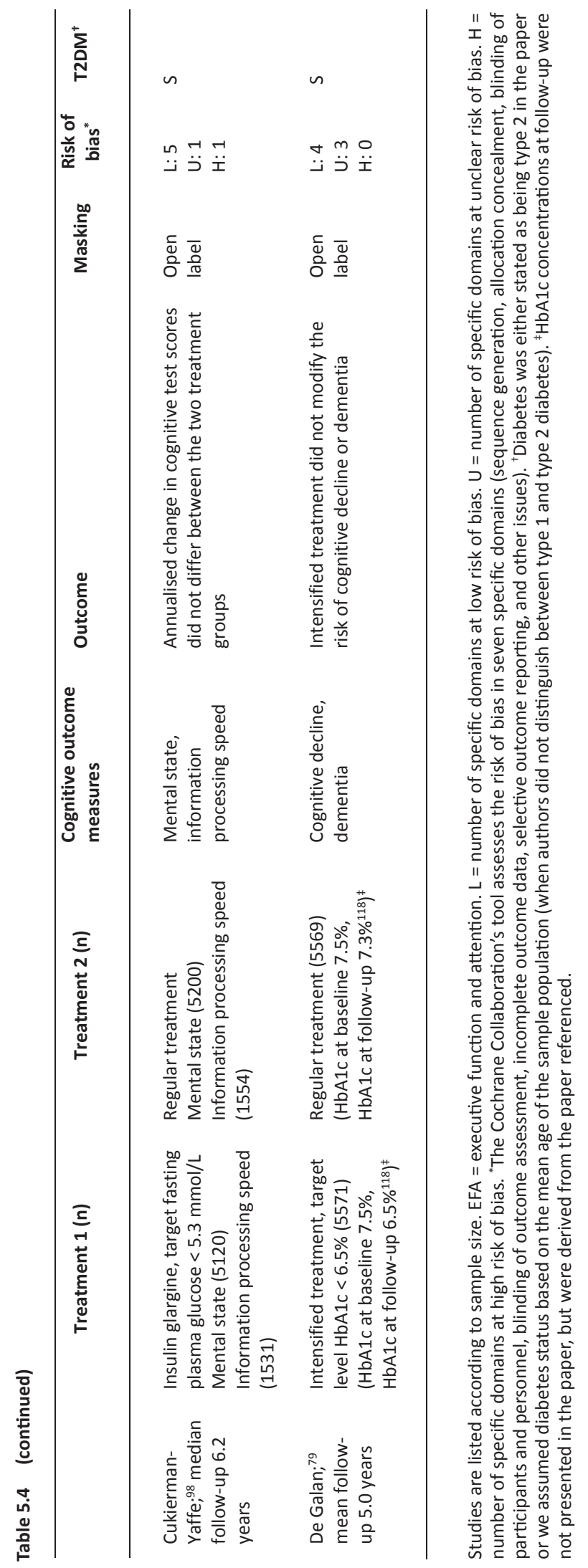


dementia $32,37,7,81,83,89,95$ (one combined dementia with CIND $^{83}$ ) and glucose-lowering treatments. From these observational studies, no consistent pattern was observed (Supplement). Six studies focused on cognitive decline, ${ }^{27,31,45,69,82,94}$ but study designs and results were heterogeneous and did not allow definite conclusions.

Two randomised controlled trials involved head-to-head comparison of the effect of different glucose-lowering drugs on glucose regulation, and potentially on cerebral outcomes (Table 5.4). One trial compared 24 weeks of rosiglitazone with glyburide treatment (based as add-on therapy to metformin). ${ }^{61}$ Working memory improved in both treatment groups and there was no significant difference between the two groups. The other trial compared 12 months of repaglinide with glibenclamide treatment; only glibenclamide was associated with cognitive decline. ${ }^{60}$

One trial compared whether titrated insulin glargine versus standard care affected cognitive decline. ${ }^{98}$ Patients with type 2 diabetes using more than one oral glucoselowering drug were excluded from the trial. During 6.2 years of follow-up, annualised decline in cognitive function and information processing speed did not differ between the two groups.

Four trials assessed the effects of intensified versus regular glucose-lowering treatment on cognitive function (Table 5.4). ${ }^{29,33,36,79}$ Target $\mathrm{HbA} 1 \mathrm{c}$ concentrations for intensified treatment varied between the trials $\left(<6 \%[42 \mathrm{mmol} / \mathrm{mol}],{ }^{33}<6.5 \%[48 \mathrm{mmol} / \mathrm{mol}],{ }^{79}<7 \%\right.$ [53 mmol/mol], ${ }^{29}$ and $<7$ to $8 \%$ [53 to $\left.\left.64 \mathrm{mmol} / \mathrm{mol}\right]^{36}\right)$. Although target concentrations were not achieved in all trials, there was a difference in $\mathrm{HbA1c}$ concentrations between the two treatment groups (ranging from $0.3 \%$ to $0.9 \%$ ). Only one trial, ${ }^{36}$ in which the intervention was based on a telemedicine coaching programme, reported that intensified treatment had more positive effects on cognitive function than did regular treatment. The other trials showed no benefit of intensified treatment. In two trials, ${ }^{29,33}$ positive effects on cognitive function compared with baseline performance were seen in both treatment groups, but differences between the two groups were not significant.

\section{Discussion}

This systematic review provides evidence that measures of glycaemia, particularly high concentrations of $\mathrm{HbAlc}$ and glucose variability, are negatively associated with cognitive function in people with type 2 diabetes without dementia. However, the strength of this association is weak, with an explained variance of HbA1c of mostly below $10 \%$. Uncertainty remains about the association between cognitive function and other measures of glucose regulation (ie, insulin concentration, insulin resistance, 
and hypoglycaemic events). Our review also showed that there are limited data about long-term cerebral outcomes, such as dementia and structural changes on MRI, and the effects of glucose-lowering treatment on these outcomes. Figure 5.2 shows a summary of the findings of this review; the findings with exclusion of studies at high risk of bias are shown in the Supplement.

To our knowledge, apart from a Cochrane systematic review ${ }^{108}$ on the effects of glucoselowering treatment on cognitive function, this is the first systematic review to evaluate the associations between different measures of glucose regulation and cognitive function and brain abnormalities on MRI in people with type 2 diabetes. We emphasise that our intention in this review was not to compare these parameters in people with diabetes and those without diabetes. Our primary aim was to explore the links between glucose regulation and the severity and progression of cerebral complications in people with type 2 diabetes. To identify all relevant papers, a broad literature search was done. We included several measures of glucose regulation, because these measures are interrelated, but each provides different information. We also used several outcome measures for cognitive function and structural brain abnormalities on MRI. The main restriction we applied in the selection of studies -based on our knowledge of the literature- was a sample size of at least 40 patients, because smaller studies are likely to be underpowered. The weak associations reported in this review justify the use of a sample size criterion. Moreover, this review summarises published data and the use of a sample size might have limited distortion of the findings by the general tendency of small negative studies to remain unpublished.

Some methodological issues deserve condiseration. First, the included studies were heterogeneous in terms of participants, measures of glucose regulation, cognitive outcomes, and statistical analyses. Although this is partly due to our broad search strategy, a formal meta-analysis could not be done, even when subsets of determinants and outcomes were considered. A qualitative comparison showed no systematic differences in the results of the included studies according to demographic and diabetes-specific characteristics. In fact, the consistency of the results in different study populations favours the generalisability of our findings. Second, few studies included vascular risk factors (eg, blood pressure) or comorbid conditions (eg, depression) as covariates in their statistical models. Thus, we cannot say whether the observed associations are causal or can be explained by confounding or mediating effects of other factors that were not sufficiently taken into account. For example, hypertension, particularly in midlife, has been associated with decrements in cognitive function. ${ }^{15,109}$ Third, most studies failed to take into consideration non-linearity of the associations between markers of glucose regulation and cognitive function or brain abnormalities on MRI. Consequently, 
studies might have been unable to detect such associations, which might lead to a slight underestimation of the role of glucose regulation in cognitive function in people with type 2 diabetes.

Of measures of glucose regulation, $\mathrm{HbA1c}$ concentration was studied most frequently and consistently across studies. HbA1c concentration reflects the mean glucose concentration over a period of 8 to 12 weeks, resulting from both fasting and postprandial glucose concentrations ${ }^{110}$ and provides clinically meaningful information about longterm glycaemic control in people with type 2 diabetes. A substantial number of studies also assessed fasting blood glucose concentrations, which mainly reflect nocturnal hepatic gluconeogenesis, influenced by hepatic insulin sensitivity. Given the intraindividual day-to-day variability of fasting blood glucose concentrations, ${ }^{111}$ it is important to mention that most studies did not report whether blood sampling and cognitive assessment took place on the same day. Other measures of glucose regulation were assessed less frequently or less consistently than $\mathrm{HbA} 1 \mathrm{c}$ and fasting blood glucose. For example, postprandial or post-load blood glucose concentrations, which reflect insulin secretory responses to different stimuli, ${ }^{112}$ were used to asses non-fasting glycaemia. To assess insulin resistance, studies generally used surrogate indices, such as fasting insulin concentrations and HOMA-IR.

Cognitive outcomes examined in this review included both cognitive status (ie, cognitive performance at a single point in time) and changes in cognitive performance over time, which ranged from subtle cognitive changes to incident dementia. Most studies focused on subtle cognitive changes, using various tests and screening methods to assess specific aspects of cognitive function. We categorised these tests according to the cognitive domains they assessed, using an established approach. ${ }^{15,113}$ The present review shows that measures of glycaemia are negatively, but weakly, associated with scores on cognitive function tests in people with type 2 diabetes. This association is particularly true for increased $\mathrm{HbA} 1 \mathrm{c}$ concentration and acute glucose fluctuations, whereas fasting blood glucose concentrations tend to be unrelated to cognitive function in people with type 2 diabetes. The relation between long-term glycaemic control (reflected by $\mathrm{HbA1C}$ concentrations) and cognitive status might indicate adverse effects of prolonged hyperglycaemia on the brain, although reverse causality needs to be considered, whereby people with poor cognitive abilities that might be independent of diabetes have difficulty maintaining glycaemic control. The relation between glucose variability and cognitive function in the short term might reflect other processes, possibly changes in osmotic pressure or other short-term metabolic effects. The available evidence does not allow conclusions to be drawn about the association between measures of insulin concentration and insulin resistance and cognitive test performance in people with type 2 diabetes. 
Figure 5.2 Summary of results of included studies

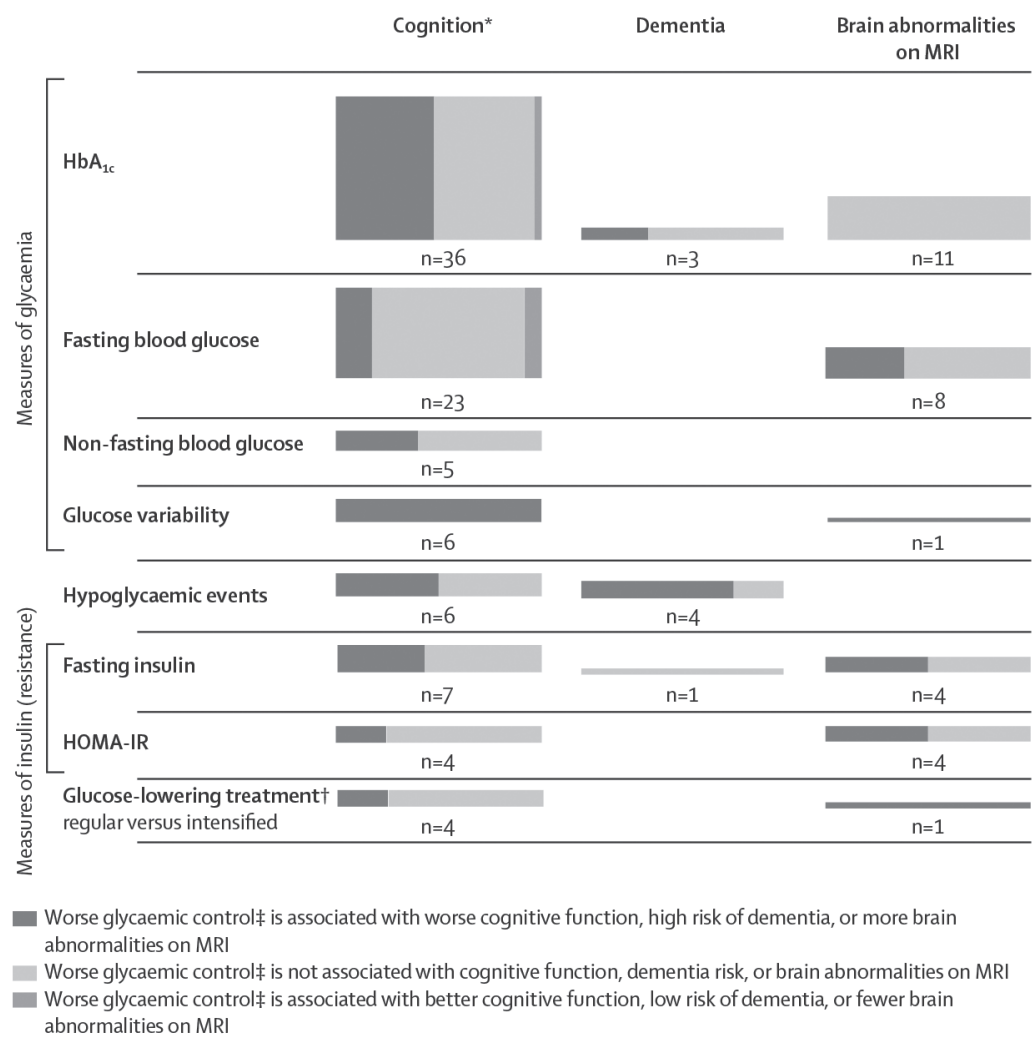

A summary of results excluding studies at high risk of bias is shown in the Supplement. Results are derived from statistical models controlled for demographic factors en vascular risk factors (when applicable). The height of each bar indicates the amount of evidence available. Results of cross-sectional and longitudinal analyses are combined. ${ }^{*}$ Cognition includes measures that reflect cognitive status (cognitive function at a single point in time) or change in cognitive function over time. 'Intensified glucose-lowering treatment was associated with less decline in total brain volume, but more progression of white matter hyperintensities on MRI. ${ }^{\ddagger}$ Worse glycaemic controles is defined as high concentrations of $\mathrm{HbA} 1 \mathrm{c}$, fasting blood glucose, or non-fasting blood glucose; greater glucose variability; hypoglycaemic events; high fasting insulin concentration; high HOMA-IR; or achieved with glucose-lowering treatment. HOMA-IR = homeostasis model assessment of insulin resistance. 
Cognitive decline in people with type 2 diabetes is thought to progress slowly over time compared with people without diabetes. ${ }^{1}$ Thus, the ability to detect associations between measures of glucose regulation and changes in cognitive function over time is generally difficult. Not surprisingly, therefore, studies have shown inconsistent results on the association between measures of glucose regulation and cognitive decline. Brain abnormalities on MRI might serve as a proxy for long-term cognitive outcomes and the future risk of dementia. Results of the few studies focusing on the association between structural brain abnormalities and glucose regulation were inconclusive, although several studies found an association between fasting blood glucose concentrations and brain volume, possibly reflecting osmotic effects of short-term hyperglycaemia on brain tissue. $\mathrm{HbA1c}$ concentration was generally unrelated to brain abnormalities on MRI.

Although dementia is clearly a clinically relevant long-term outcome, this review shows that information about its association with measures of glucose regulation in type 2 diabetes is scarce. Evidence exists to supports an association between hypoglycaemic events and increased risk of dementia. Additionally, an $\mathrm{HbA1c}$ concentration higher than $10 \%$ ( $86 \mathrm{mmol} / \mathrm{mol}$ ) has been proven to increase the risk of development of dementia. ${ }^{89}$

As the population ages, and the prevalence of diabetes continues to grow, an increasing number of people will face the cerebral complications of diabetes. Prevention of cognitive decline, and dementia, should therefore become important treatment targets for people with type 2 diabetes. ${ }^{1}$ However, evidence on the potential benefits of glucose-lowering treatment is limited to observational studies and a handful of randomised controlled trials. Observational studies have compared a wide variety of glucose-lowering drugs or treatment modalities, and results showed no consistent pattern. In addition, confounding by indication can lead to bias, limiting the interpretation of results. Randomised controlled trials have mainly explored the effect of intensified versus regular glycaemic control on cognitive function. In these trials, cognitive function was often a secondary outcome. Although all trials recorded a difference in $\mathrm{HbA1c}$ concentration between treatment groups, intensified treatment was not generally associated with improved cognitive function. The question is does the slow decline in cognitive function in people with type 2 diabetes leave room for observing a treatment effect, even in studies with several years of follow-up? Another issue is that the slight cognitive impairments that are associated with type 2 diabetes and the increased risk of dementia might not be part of a continuum. ${ }^{1}$ Mean scores on cognitive function tests mainly relate to the slight cognitive impairment associated with type 2 diabetes, whereas the incidence of dementia or accelerated cognitive decline might be a more relevant clinical outcome with a different underlying pathophysiological mechanism. Nonetheless, positive effects on cognitive function were observed in both treatment groups in most trials, suggesting 
that improving glycaemic control does have beneficial effects on brain function.

Because this review indicates that the role of hyperglycaemia in cognition is limited, other factors that might contribute to the onset and progression of cerebral changes in people with type 2 diabetes need to be considered. Vascular risk factors (eg, hypertension, dyslipidaemia, and obesity) are associated with ageing-related cognitive decline in the general population ${ }^{12,13}$ and are more prevalent among people with type 2 diabetes. Indeed, the risk of cognitive decline in people with type 2 diabetes has been shown to increase with high systolic blood pressure. ${ }^{113}$ Moreover, microvascular changes in the retina have been linked to cognitive impairment in people with diabetes, ${ }^{114}$ supporting the hypothesis that vascular pathology is involved in the cerebral complications of type 2 diabetes. Other possible risk factors are low-grade inflammation and abnormalities in the hypothalamic-pituitary-adrenal axis. ${ }^{1}$ Recommendations for future research are in Panel 5.2.

This present review shows that in people with type 2 diabetes, measures of glycaemia are negatively, but weakly, associated with cognitive function. At present, insight into the role of insulin in the cerebral complications of type 2 diabetes is incomplete, although cerebral effects of insulin are increasingly recognised. ${ }^{11}$ Further randomised controlled trials are needed to establish whether glucose-lowering treatment prevents cognitive decline in people with type 2 diabetes in the long term. 


\section{Panel 5.2 - Recommendations for future research}

Numerous neuropsychological tests are used to assess subtle cognitive changes in people with type 2 diabetes, complicating the comparison of data. Harmonisation of neuropsychological tests across future studies is strongly recommended to increase coherence in the acquired data. Cognitive domains that should be assessed are memory, executive function and attention, and information processing speed. A recent systematic review provides guidance on the sensitivity of tests. ${ }^{115}$

Apart from changes in cognitive function, long-term cerebral outcomes (ie, brain abnormalities on MRI, accelerated cognitive decline, or dementia) are of particular interest because they profoundly affect a person's functional independence and quality of life. Longitudinal studies that are statistically powered with adequate follow-up duration are needed to understand the effect of glucose regulation on long-term cerebral outcomes, including the incidence of dementia. Ideally, studies should explore diverse aspects of glucose regulation, beyond $\mathrm{HbA} 1 \mathrm{c}$ and fasting glucose concentrations, and can thereby adequately adjust effect sizes for potential confounding effects of several demographic factors (ie, age, sex, and education level) and comorbid conditions (ie, hypertension and depression).

Little is known about the effect of different types of glucose-lowering treatment on brain structure and function. Treatment effects might not be restricted to the glucose-lowering effects of these drugs, but might include pleiotropic effects directly on the brain. ${ }^{116}$ Cognitive function is a clinically relevant outcome measure in trials of glucose-lowering drugs in patients with type 2 diabetes. Future trials should focus on accelerated cognitive decline or dementia as cognitive outcomes, ${ }^{1}$ and investigators should consider including patients who are at high risk of these outcomes (ie, elderly patients with cardiovascular comorbidities). ${ }^{89}$ 


\section{References}

1. Biessels GJ, Strachan MWJ, Visseren FLJ, Kappelle LJ, Whitmer RA. Dementia and cognitive decline in type 2 diabetes and prediabetic stages: towards targeted interventions. The Lancet Diabetes \& Endocrinology 2013; (0).

2. Cheng $G$, Huang $C$, Deng $H$, Wang $H$. Diabetes as a risk factor for dementia and mild cognitive impairment: a meta-analysis of longitudinal studies. Intern Med J 2012; 42(5): 484-91.

3. Van Harten B, de Leeuw FE, Weinstein HC, Scheltens P, Biessels GJ. Brain imaging in patients with diabetes: a systematic review. Diabetes Care 2006; 29(11): 2539-48.

4. Biessels GJ, Reijmer YD. Brain changes underlying cognitive dysfunction in diabetes: what can we learn from MRI? Diabetes 2014; 63: 2244-52.

5. Antonetti DA, Klein R, Gardner TW. Diabetic retinopathy. N Engl J Med 2012; 366(13): 122739.

6. Callaghan BC LA, Feldman EL, Hughes RAC. Enhanced glucose control for preventing and treating diabetic neuropathy. Cochrane Database of Systematic Reviews 2012; 6.

7. Chait A, Bornfeldt KE. Diabetes and atherosclerosis: is there a role for hyperglycemia? Journal of lipid research 2009; 50 Suppl: S335-9.

8. Sima AA, Kamiya H, Li ZG. Insulin, C-peptide, hyperglycemia, and central nervous system complications in diabetes. Eur J Pharmacol 2004; 490(1-3): 187-97.

9. Biessels GJ, Staekenborg S, Brunner E, Brayne C, Scheltens P. Risk of dementia in diabetes mellitus: a systematic review. Lancet Neurol 2006; 5(1): 64-74.

10. Last D, Alsop DC, Abduljalil AM, et al. Global and regional effects of type 2 diabetes on brain tissue volumes and cerebral vasoreactivity. Diabetes Care 2007; 30(5): 1193-9.

11. Cholerton B, Baker LD, Craft S. Insulin, cognition, and dementia. Eur J Pharmacol 2013; 719(13): $170-9$.

12. Plassman BL, Williams JW, Jr., Burke JR, Holsinger T, Benjamin S. Systematic review: factors associated with risk for and possible prevention of cognitive decline in later life. Ann Intern Med 2010; 153(3): 182-93.

13. Kloppenborg RP, van den Berg E, Kappelle LJ, Biessels GJ. Diabetes and other vascular risk factors for dementia: which factor matters most? A systematic review. Eur J Pharmacol 2008; 585(1): 97-108.

14. Moher D, Liberati A, Tetzlaff J, Altman DG, Group P. Preferred reporting items for systematic reviews and meta-analyses: the PRISMA statement. BMJ 2009; 339: b2535.

15. Van den Berg E, Kloppenborg RP, Kessels RP, Kappelle LJ, Biessels GJ. Type 2 diabetes mellitus, hypertension, dyslipidemia and obesity: A systematic comparison of their impact on cognition. Biochim Biophys Acta 2009; 1792(5): 470-81.

16. Centers for Disease Control and Prevention. National diabetes fact sheet: national estimates and general information on diabetes and prediabetes in the United States, 2011. http://www. cdc.gov/diabetes/pubs/pdf/ndfs_2011.pdf (accessed June 8, 2014).

17. Brundel M, Kappelle LJ, Biessels GJ. Brain imaging intype 2 diabetes. Eur Neuropsychopharmacol 2014.

18. Schmidt IW, Berg IJ, Deelman BG. Relations between subjective evaluations of memory and objective memory performance. Percept Mot Skills 2001; 93(3): 761-76.

19. Wardlaw JM, Smith EE, Biessels GJ, et al. Neuroimaging standards for research into small vessel disease and its contribution to ageing and neurodegeneration. Lancet Neurol 2013; 12(8): 822-38.

20. Wells GA, Shea B, O'Connell D, et al. The Newcastle-Ottawa Scale (NOS) for assessing the quality of nonrandomised studies in meta-analyses. 2006. http://www.ohri.ca/programs/ clinical_epidemiology/nos_manual.pdf (accessed Jan 10, 2014).

21. Higgins JP, Altman DG, Gotzsche PC, et al. The Cochrane Collaboration's tool for assessing risk of bias in randomised trials. BMJ 2011; 343: d5928. 
22. Lin $\mathrm{CH}$, Sheu WH. Hypoglycaemic episodes and risk of dementia in diabetes mellitus: 7-year follow-up study. J Intern Med 2012.

23. Pearce KL, Noakes M, Wilson C, Clifton PM. Continuous Glucose Monitoring and Cognitive Performance in Type 2 Diabetes. Diabetes Technol Ther 2012; 14(12): 1126-33.

24. Mukherjee P, Mazumdar S, Goswami S, et al. Cognitive dysfunction in diabetic patients with special reference to age of onset, duration and control of diabetes. Activitas Nervosa Superior 2012; 54(1-2): 67-75.

25. Benedict C, Kilander L, Brooks SJ, et al. Impaired insulin sensitivity as indexed by the HOMA score is associated with deficits in verbal fluency and temporal lobe gray matter volume in the elderly. Diabetes Care 2012; 35(3): 488-94.

26. Zhong $Y$, Zhang $X Y$, Miao $Y$, et al. The relationship between glucose excursion and cognitive function in aged type 2 diabetes patients. Biomedical and Environmental Sciences 2012; 25(1): 1-7.

27. Umegaki $H$, limuro $S$, Shinozaki $T$, et al. Risk factors associated with cognitive decline in the elderly with type 2 diabetes: Baseline data analysis of the Japanese elderly diabetes intervention trial. Geriatrics and Gerontology International 2012; 12(SUPPL.1): 103-9.

28. Aung PP, Strachan MWJ, Frier BM, Butcher I, Deary IJ, Price JF. Severe hypoglycaemia and late-life cognitive ability in older people with Type2 diabetes: The Edinburgh Type2 Diabetes Study. Diabetic Medicine 2012; 29(3): 328-36.

29. Koekkoek PS, Ruis C, Van Den Donk M, et al. Intensive multifactorial treatment and cognitive functioning in screen-detected type 2 diabetes - The ADDITION-Netherlands study: A clusterrandomized trial. Journal of the Neurological Sciences 2012; 314(1-2): 71-7.

30. Hayashi K, Kurioka S, Yamaguchi T, et al. Association of cognitive dysfunction with hippocampal atrophy in elderly Japanese people with type 2 diabetes. Diabetes Research and Clinical Practice 2011; 94(2): 180-5.

31. Espeland MA, Miller ME, Goveas JS, et al. Cognitive function and fine motor speed in older women with diabetes mellitus: Rresults from the women's health initiative study of cognitive aging. Journal of Women's Health 2011; 20(10): 1435-43.

32. Hsu CC, Wahlqvist ML, Lee MS, Tsai HN. Incidence of dementia is increased in type 2 diabetes and reduced by the use of sulfonylureas and metformin. Journal of Alzheimer's Disease 2011; 24(3): 485-93.

33. Launer L, Miller ME, Williamson JD, et al. Effects of intensive glucose lowering on brain structure and function in people with type 2 diabetes (ACCORD MIND): A randomised openlabel substudy. The Lancet Neurology 2011; 10(11): 969-77.

34. Imamine R, Kawamura T, Umemura T, et al. Does cerebral small vessel disease predict future decline of cognitive function in elderly people with type 2 diabetes? Diabetes Research and Clinical Practice 2011; 94(1): 91-9.

35. Mahakaeo S, Zeimer H, Woodward M. Relationship between glycemic control and cognitive function in patients with type 2 diabetes in a hospital aged care unit. European Geriatric Medicine 2011; 2(4): 204-7.

36. Luchsinger JA, Palmas W, Teresi JA, et al. Improved diabetes control in the elderly delays global cognitive decline. Journal of Nutrition, Health and Aging 2011; 15(6): 445-9.

37. Parikh NM, Morgan RO, Kunik ME, et al. Risk factors for dementia in patients over 65 with diabetes. International Journal of Geriatric Psychiatry 2011; 26(7): 749-57.

38. Reijmer YD, Van Den Berg E, De Bresser J, et al. Accelerated cognitive decline in patients with type 2 diabetes: MRI correlates and risk factors. Diabetes/Metabolism Research and Reviews 2011; 27(2): 195-202.

39. Kadoi $Y$, Kawauchi $C$, Ide M, et al. Preoperative depression is a risk factor for postoperative short-term and long-term cognitive dysfunction in patients with diabetes mellitus. Journal of Anesthesia 2011; 25(1): 10-7.

40. Rizzo MR, Marfella R, Barbieri M, et al. Relationships between daily acute glucose fluctuations 
and cognitive performance among aged type 2 diabetic patients. Diabetes Care 2010; 33(10): 2169-74.

41. Reynolds RM, Strachan MWJ, Labad J, et al. Morning cortisol levels and cognitive abilities in with type 2 diabetes: The Edinburgh type 2 diabetes study. Diabetes Care 2010; 33(4): 71420.

42. Abbatecola AM, Lattanzio F, Molinari AM, et al. Rosiglitazone and cognitive stability in older individuals with type 2 diabetes and mild cognitive impairment. Diabetes Care 2010; 33(8): 1706-11.

43. Alencar RC, Cobas RA, Gomes MB. Assessment of cognitive status in patients with type 2 diabetes through the mini-mental status examination: A cross-sectional study. Diabetology and Metabolic Syndrome 2010; 2(1).

44. Shimada H, Miki T, Tamura A, Ataka S, Emoto M, Nishizawa Y. Neuropsychological status of elderly patients with diabetes mellitus. Diabetes Research and Clinical Practice 2010; 87(2): 224-7.

45. Plastino M, Fava A, Pirritano D, et al. Effects of insulinic therapy on cognitive impairment in patients with Alzheimer disease and Diabetes Mellitus type-2. Journal of the Neurological Sciences 2010; 288(1-2): 112-6.

46. Perlmuter LC, Shah PH, Flanagan BP, et al. Rate of peripheral glucose change during cognitive testing predicts performance in diabetes mellitus. Journal of diabetes 2009; 1(1): 43-9.

47. Whitmer RA, Karter AJ, Yaffe K, Quesenberry Jr CP, Selby JV. Hypoglycemic episodes and risk of dementia in older patients with type 2 diabetes mellitus. JAMA : the journal of the American Medical Association 2009; 301(15): 1565-72.

48. Cukierman-Yaffe T, Gerstein HC, Williamson JD, et al. Relationship between baseline glycemic control and cognitive function in individuals with type 2 diabetes and other cardiovascular risk factors: the action to control cardiovascular risk in diabetes-memory in diabetes (ACCORDMIND) trial. Diabetes care 2009; 32(2): 221-6.

49. Dore GA, Elias MF, Robbins MA, Elias PK, Nagy Z. Presence of the APOE 4 allele modifies the relationship between type 2 diabetes and cognitive performance: The Maine-Syracuse Study. Diabetologia 2009; 52(12): 2551-60.

50. Bruce DG, Davis WA, Casey GP, et al. Severe hypoglycaemia and cognitive impairment in older patients with diabetes: The Fremantle Diabetes Study. Diabetologia 2009; 52(9): 1808-15.

51. Tiehuis AM, Mali WPTM, van Raamt AF, et al. Cognitive dysfunction and its clinical and radiological determinants in patients with symptomatic arterial disease and diabetes. Journal of the Neurological Sciences 2009; 283(1-2): 170-4.

52. Umegaki H, Kawamura T, Mogi N, Umemura T, Kanai A, Sano T. Glucose control levels, ischaemic brain lesions, and hyperinsulinaemia were associated with cognitive dysfunction in diabetic elderly. Age Ageing 2008; 37(4): 458-61.

53. Saczynski JS, Jonsdottir MK, Garcia ME, et al. Cognitive impairment: an increasingly important complication of type 2 diabetes: the age, gene/environment susceptibility--Reykjavik study. American Journal of Epidemiology 2008; 168(10): 1132-9.

54. Kim E, Cho MH, Cha KR, et al. Interactive effect of central obesity and hypertension on cognitive function in older out-patients with type 2 diabetes. Diabetic Medicine 2008; 25(12): 1440-6.

55. Obidi CS, Pugeda JP, Fan X, et al. Race moderates age-related cognitive decline in type 2 diabetes. Experimental Aging Research 2008; 34(2): 114-25.

56. Dickinson D, Gold JM, Dickerson FB, Medoff D, Dixon LB. Evidence of exacerbated cognitive deficits in schizophrenia patients with comorbid diabetes. Psychosomatics 2008; 49(2): 12331.

57. Irie F, Fitzpatrick AL, Lopez OL, et al. Enhanced risk for Alzheimer disease in persons with type 2 diabetes and APOE 4: The cardiovascular health study cognition study. Archives of Neurology 2008; 65(1): 89-93. 
58. Manschot SM, Biessels GJ, De Valk H, et al. Metabolic and vascular determinants of impaired cognitive performance and abnormalities on brain magnetic resonance imaging in patients with type 2 diabetes. Diabetologia 2007; 50(11): 2388-97.

59. Van Harten B, Oosterman J, Muslimovic D, van Loon BJP, Scheltens P, Weinstein HC. Cognitive impairment and MRI correlates in the elderly patients with type 2 diabetes mellitus. Age and Ageing 2007; 36(2): 164-70.

60. Abbatecola AM, Rizzo MR, Barbieri M, et al. Postprandial plasma glucose excursions and cognitive functioning in aged type 2 diabetics. Neurology 2006; 67(2): 235-40.

61. Ryan CM, Freed MI, Rood JA, Cobitz AR, Waterhouse BR, Strachan MWJ. Improving metabolic control leads to better working memory in adults with type 2 diabetes. Diabetes Care 2006; 29(2): 345-51.

62. Manschot SM, Brands AM, van der Grond J, et al. Brain magnetic resonance imaging correlates of impaired cognition in patients with type 2 diabetes. Diabetes 2006; 55(4): 1106-13.

63. Akisaki T, Sakurai T, Takata $\mathrm{T}$, et al. Cognitive dysfunction associates with white matter hyperintensities and subcortical atrophy on magnetic resonance imaging of the elderly diabetes mellitus Japanese elderly diabetes intervention trial (J-EDIT). Diabetes/Metabolism Research and Reviews 2006; 22(5): 376-84.

64. Shorr RI, De Rekeneire N, Resnick HE, et al. Glycemia and cognitive function in older adults using glucose-lowering drugs. Journal of Nutrition, Health and Aging 2006; 10(4): 297-301.

65. Van den Berg E, De Craen AJM, Biessels GJ, Gussekloo J, Westendorp RGJ. The impact of diabetes mellitus on cognitive decline in the oldest of the old: A prospective populationbased study. Diabetologia 2006; 49(9): 2015-23.

66. Gallacher JEJ, Pickering J, Elwood PC, Bayer AJ, Yarnell JW, Ben-Shlomo Y. Glucoregulation has greater impact on cognitive performance than macro-vascular disease in men with type 2 diabetes: Data from the Caerphilly study. European Journal of Epidemiology 2005; 20(9): 761-8.

67. Mogi N, Umegaki $\mathrm{H}$, Hattori A, et al. Cognitive function in Japanese elderly with type 2 diabetes mellitus. Journal of Diabetes and its Complications 2004; 18(1): 42-6.

68. Logroscino G, Kang JH, Grodstein F. Prospective study of type 2 diabetes and cognitive decline in women aged 70-81 years. British Medical Journal 2004; 328(7439): 548-51.

69. Wu JH, Haan MN, Liang J, Ghosh D, Gonzalez HM, Herman WH. Impact of antidiabetic medications on physical and cognitive functioning of older Mexican Americans with diabetes mellitus: A population-based cohort study. Annals of Epidemiology 2003; 13(5): 369-76.

70. Crooks VC, Buckwalter JG, Petitti DB. Diabetes mellitus and cognitive performance in older women. Annals of Epidemiology 2003; 13(9): 613-9.

71. De Luis DA, Fernandez N, Arranz M, Aller R, Izaola O. Total homocysteine and cognitive deterioration in people with type 2 diabetes. Diabetes Research and Clinical Practice 2002; 55(3): 185-90.

72. Vanhanen $\mathrm{M}$, Kuusisto J, Koivisto $\mathrm{K}$, et al. Type-2 diabetes and cognitive function in a nondemented population. Acta Neurologica Scandinavica 1999; 100(2): 97-101.

73. Naor M, Steingruber HJ, Westhoff K, Schottenfeld-Naor Y, Gries AF. Cognitive function in elderly non-insulin-dependent diabetic patients before and after inpatient treatment for metabolic control. Journal of Diabetes and its Complications 1997; 11(1): 40-6.

74. Worrall GJ, Chaulk PC, Moulton N. Cognitive function and glycosylated hemoglobin in older patients with type II diabetes. Journal of Diabetes and its Complications 1996; 10(6): 320-4.

75. Zaslavsky LMA, Gross JL, Chaves ML, Machado R. Memory dysfunction and autonomic neuropathy in non-insulin-dependent (type 2) diabetic patients. Diabetes Research and Clinical Practice 1995; 30(2): 101-10.

76. Kalmijn S, Feskens EJM, Launer LJ, Stijnen T, Kromhout D. Glucose intolerance, hyperinsulinaemia and cognitive function in a general population of elderly men. Diabetologia 1995; 38(9): 1096-102. 
77. Lowe LP, Tranel D, Wallace RB, Welty TK. Type II diabetes and cognitive function: A populationbased study of Native Americans. Diabetes Care 1994; 17(8): 891-6.

78. Malacco E, Di Cesare F. Effects of dihydroergocristine treatment on carbohydrate tolerance and cognitive function in patients with non-insulin-dependent diabetes. Current Therapeutic Research - Clinical and Experimental 1992; 51(4): 515-23.

79. De Galan BE, Zoungas S, Chalmers J, et al. Cognitive function and risks of cardiovascular disease and hypoglycaemia in patients with type 2 diabetes: The action in diabetes and vascular disease: Preterax and diamicron modified release controlled evaluation (ADVANCE) trial. Diabetologia 2009; 52(11): 2328-36.

80. Zhen YF, Zhang J, Liu XY, et al. Low BDNF is associated with cognitive deficits in patients with type 2 diabetes. Psychopharmacology (Berl) 2013; 227(1): 93-100.

81. Yaffe K, Falvey $\mathrm{CM}$, Hamilton N, et al. Association between hypoglycemia and dementia in a biracial cohort of older adults with diabetes mellitus. JAMA internal medicine 2013; 173(14): 1300-6.

82. Seaquist ER, Miller ME, Fonseca $\mathrm{V}$, et al. Effect of thiazolidinediones and insulin on cognitive outcomes in ACCORD-MIND. J Diabetes Complications 2013; 27(5): 485-91.

83. Mayeda ER, Haan MN, Kanaya AM, Yaffe K, Neuhaus J. Type 2 diabetes and 10-year risk of dementia and cognitive impairment among older Mexican Americans. Diabetes Care 2013; 36(9): 2600-6.

84. Feinkohl I, Aung PP, Keller M, et al. Severe hypoglycemia and cognitive decline in older people with type 2 diabetes: the Edinburgh Type 2 Diabetes Study. Diabetes Care 2013.

85. Fei M, Yan Ping Z, Ru Juan M, Ning Ning L, Lin G. Risk factors for dementia with type 2 diabetes mellitus among elderly people in China. Age Ageing 2013; 42(3): 398-400.

86. Crane PK, Walker R, Hubbard RA, et al. Glucose levels and risk of dementia. N Engl J Med 2013; 369(6): 540-8.

87. Tuligenga RH, Dugravot A, Tabák AG, et al. Midlife type 2 diabetes and poor glycaemic control as risk factors for cognitive decline in early old age: a post-hoc analysis of the Whitehall II cohort study. The Lancet Diabetes \& Endocrinology; (0).

88. Gutierrez-Hermosillo H, Diaz De Leon-Gonzalez E, Palacios-Corona R, et al. C allele of the rs2209972 single nucleotide polymorphism of the insulin degrading enzyme gene and Alzheimer's disease in type 2 diabetes, a case control study. Medicina clinica 2013.

89. Exalto LG, Biessels GJ, Karter AJ, et al. Risk score for prediction of 10 year dementia risk in individuals with type 2 diabetes: A cohort study. The Lancet Diabetes and Endocrinology 2013; 1(3): 183-90.

90. Munshi M, Grande L, Hayes M, et al. Cognitive dysfunction is associated with poor diabetes control in older adults. Diabetes Care 2006; 29(8): 1794-9.

91. Luchsinger JA, Tang MX, Shea S, Mayeux R. Hyperinsulinemia and risk of Alzheimer disease. Neurology 2004; 63(7): 1187-92.

92. Christman AL, Matsushita K, Gottesman RF, et al. Glycated haemoglobin and cognitive decline: the Atherosclerosis Risk in Communities (ARIC) study. Diabetologia 2011; 54(7): 1645-52.

93. Umegaki $H$, Kawamura T, Umemura T, Kawano N. Factors associated with cognitive decline in older adults with type 2 diabetes mellitus during a 6-year observation. Geriatr Gerontol Int 2014.

94. Ng TP, Feng L, Yap KB, Lee TS, Tan CH, Winblad B. Long-Term Metformin Usage and Cognitive Function among Older Adults with Diabetes. J Alzheimers Dis 2014.

95. Huang CC, Chung CM, Leu HB, et al. Diabetes mellitus and the risk of Alzheimer's disease: a nationwide population-based study. PLoS One 2014; 9(1): e87095.

96. Cui X, Abduljalil A, Manor BD, Peng CK, Novak V. Multi-scale glycemic variability: a link to gray matter atrophy and cognitive decline in type 2 diabetes. PLoS One 2014; 9(1): e86284.

97. Bruce DG, Davis WA, Starkstein SE, Davis TM. Mid-life predictors of cognitive impairment and dementia in type 2 diabetes mellitus: the Fremantle diabetes study. J Alzheimers Dis 2014; 
98. Cukierman-Yaffe T, Bosch J, Diaz R, et al. Effects of basal insulin glargine and omega-3 fatty acid on cognitive decline and probable cognitive impairment in people with dysglycaemia: a subsutudy of the ORIGIN trial. Lancet Diabetes Endocrinol 2014; 2: 562-72.

99. Ravona-Springer R, Heymann A, Schmeidler J, et al. Trajectories in glycemic control over time are associated with cognitive performance in elderly subjects with type 2 diabetes. PLoS One 2014; 9: e97384.

100. Hsu JL, Chen YL, Leu JG, et al. Microstructural white matter abnormalities in type 2 diabetes mellitus: A diffusion tensor imaging study. Neurolmage 2012; 59(2): 1098-105.

101. De Bresser J, Tiehuis AM, van den Berg E, et al. Progression of cerebral atrophy and white matter hyperintensities in patients with type 2 diabetes. Diabetes care 2010; 33(6): 1309-14.

102 Anan F, Masaki T, Kikuchi H, et al. Association between plasma high-sensitivity C-reactive protein and insulin resistance and white matter lesions in Japanese type 2 diabetic patients. Diabetes Research and Clinical Practice 2010; 87(2): 233-9.

103. Brundel M, Van Den Heuvel M, De Bresser J, Kappelle LJ, Biessels GJ. Cerebral cortical thickness in patients with type 2 diabetes. Journal of the Neurological Sciences 2010; 299(12): 126-30.

104. Tiehuis AM, van der Graaf Y, Visseren FL, et al. Diabetes increases atrophy and vascular lesions on brain MRI in patients with symptomatic arterial disease. Stroke 2008; 39(5): 1600-3.

105. Kumar A, Haroon E, Darwin C, et al. Gray matter prefrontal changes in type 2 diabetes detected using MRI. Journal of Magnetic Resonance Imaging 2008; 27(1): 14-9.

106. Eguchi K, Ishikawa J, Hoshide S, Ishikawa S, Shimada K, Kario K. Impact of blood pressure vs. glycemic factors on target organ damage in patients with type 2 diabetes mellitus. Journal of clinical hypertension (Greenwich, Conn) 2006; 8(6): 404-10.

107. Bryan RN, Bilello M, Davatzikos C, et al. Effect of Diabetes on Brain Structure: The Action to Control Cardiovascular Risk in Diabetes MR Imaging Baseline Data. Radiology 2014: 131494.

108. Grimley EJ AS. Effect of the treatment of Type II diabetes mellitus on the development of cognitive impairment and dementia. Cochrane Database of Systematic Reviews 2003; (1).

109. Kennelly SP, Lawlor BA, Kenny RA. Blood pressure and dementia - a comprehensive review. Therapeutic advances in neurological disorders 2009; 2(4): 241-60.

110. Monnier L, Colette C. Contributions of fasting and postprandial glucose to hemoglobin A1C. Endocr Pract 2006; 12 Suppl 1: 42-6.

111. Ollerton RL, Playle R, Ahmed K, Dunstan FD, Luzio SD, Owens DR. Day-to-day variability of fasting plasma glucose in newly diagnosed type 2 diabetic subjects. Diabetes Care 1999; 22(3): 394-8.

112. Marena S, Montegrosso G, De Michieli F, Pisu E, Pagano G. Comparison of the metabolic effects of mixed meal and standard oral glucose tolerance test on glucose, insulin and C-peptide response in healthy, impaired glucose tolerance, mild and severe non-insulindependent diabetic subjects. Acta Diabetol 1992; 29(1): 29-33.

113. Umegaki H, limuro S, Shinozake T, et al. Risk factors associated with cognitive decline in the elderly with type 2 diabetes: pooled logistic analysis of a 6 -year observation in the Japanese Elderly Diabetes Intervention Trial. Geriatr Gerontol Int 2012; 12 (suppl 1): 110-16.

114. Heringa SM, Bouvy WH, van den Berg E, Moll AC, Kappelle LJ, Biessels GJ. Associations between retinal microvascular changes and dementia, cognitive functioning, and brain imaging abnormalities: a systematic review. J Cereb Blood Flow Metab 2013; 33(7): 983-95.

115. Palta P, Schneider AL, Biessels GJ, Touradji P, Hill-Briggs F. Magnitude of cognitive dysfunction in adults with type 2 diabetes: a meta-analysis of six cognitive domains and the most frequently reported neuropsychological tests within domains. J Int Neuropsychol Soc 2014; 20(3): 278-91.

116. Patrone C, Eriksson O, Lindholm D. Diabetes drugs and neurological disorders: new views and therapeutic possibilities. The lancet Diabetes \& endocrinology 2014; 2(3): 256-62.

117. Shea S, Weinstock RS, Teresi JA, et al. A randomized trial comparing telemedicine case 
management with usual care in older, ethnically diverse, medically underserved patients with diabetes mellitus: 5 year results of the IDEATel study. Journal of the American Medical Informatics Association : JAMIA 2009; 16(4): 446-56.

118. Heller SR, Group AC. A summary of the ADVANCE Trial. Diabetes Care 2009; 32 Suppl 2: S35761. 


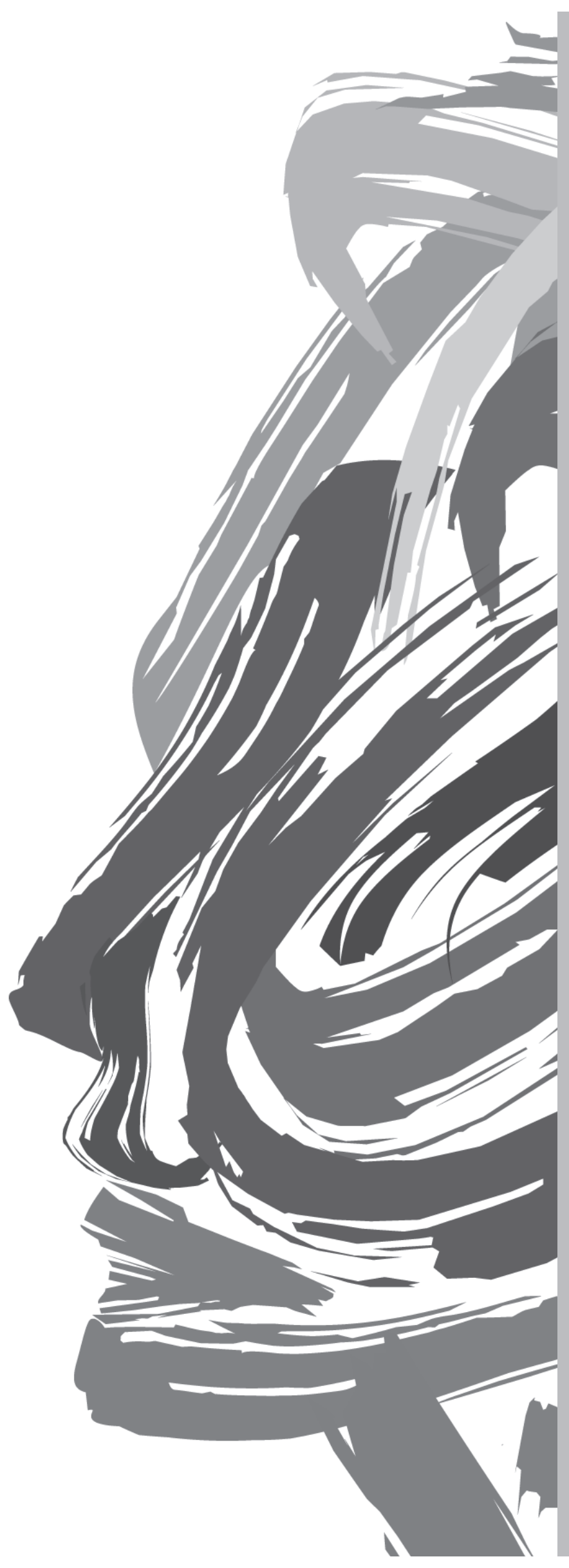


Supplement to Chapter 5 


\section{Search strategy}

Medline (PubMed) and Embase were searched to identify relevant hits. The searches performed in Medline and Embase were essentially similar, except for the subject headings. The search strategy for both databases is presented below.

The search strategy consisted of three parts. First, a search strategy was created to identify studies that investigated cognitive functioning and/or structural brain MRI abnormalities. A bottom-up approach was used to increase the sensitivity of the search. A set of subject headings (explode or non-exploded) was extended by including free search terms one by one, while continuously checking if valuable hits were added to the search. These free search terms were limited to title and abstract. Second, free search terms were used to identify studies focusing on (a subgroup of) participants with T2DM. This part of the search was limited to the titles only and included free search terms related to T2DM pathophysiology or medication (diabetes, T2DM, type 2 DM, DM type 2, glucose, insulin, glycaemic, glycemic and antidiab*). Last, the different parts of the search strategy were combined as follows: (cognitive dysfunction OR brain MRI abnormalities) AND T2DM. The search did not include additional terms related to glucose regulation to avoid missing relevant articles without these terms in the title or abstract.

\section{Search strategy for Medline (PubMed):}

("diabetes"[Title] OR "glucose"[Title] OR “insulin"[Title] OR "glycaemic"[Title] OR "glycemic"[Title] OR antidiab*[Title]) AND (("Cognition"[Mesh:noexp] OR "Attention"[Mesh] OR “Executive Function"[Mesh] OR "Learning"[Mesh:noexp] OR "Verbal Learning"[Mesh] OR "Memory"[Mesh] OR "Visual Perception"[Mesh:noexp] OR “Cognition Disorders"[Mesh:noexp] OR "Mild Cognitive Impairment"[Mesh] OR "Dementia"[Mesh] OR "cognition"[tiab] OR "cognitive"[tiab] OR "neurocognitive"[tiab] OR "neuropsychologic"[tiab] OR "neuropsychological"[tiab] OR "Mini Mental State Examination"[tiab] OR "MMSE"[tiab] OR ("executive function"[tiab] OR "executive functions"[tiab] OR "executive functioning"[tiab]) OR ("learning"[tiab] AND "hippocampus"[All Fields]) OR "memory"[tiab] OR "visual perception"[tiab] OR "dementia"[tiab] OR "Alzheimer"[tiab] OR "Alzheimer's"[tiab]) OR ((“Magnetic Resonance Imaging"[Mesh:noexp] AND "Brain"[Mesh]) OR (“Magnetic Resonance Angiography"[Mesh] AND "Brain"[Mesh]) OR ("Neuroimaging"[Mesh]) OR ("Cerebral Infarction/radiography"[Mesh]) OR ("Cerebral Small Vessel Diseases/ radiography"[Mesh]) OR ("brain"[Title/Abstract] AND "magnetic resonance imaging"[Title/Abstract]) OR ("cerebral"[Title/Abstract] AND "magnetic resonance imaging"[Title/Abstract]) OR ("brain"[Title/Abstract] AND "MRI"[Title/Abstract]) OR ("cerebral"[Title/Abstract] AND "MRI"[Title/Abstract]) OR ("brain volume"[Title/ 
Abstract]) OR ("brain atrophy"[Title/Abstract]) OR ("cerebral atrophy"[Title/Abstract]) OR ("hippocampal volume"[Title/Abstract]) OR ("hippocampus"[Title/Abstract] AND "volume"[Title/Abstract]) OR ("white matter"[Title/Abstract]) OR ("brain"[Title/ Abstract] AND "small vessel"[Title/Abstract]) OR ("cerebral"[Title/Abstract] AND "small vessel"[Title/Abstract]) OR ((brain[Title/Abstract]) AND (infarct*[Title/Abstract])) OR ((cerebral[Title/Abstract]) AND (infarct*[Title/Abstract])) OR ("lacunar"[Title/Abstract]) OR ("brain"[Title/Abstract] AND “microangiopathy"[Title/Abstract]) OR ("cerebral”[Title/ Abstract] AND "microangiopathy"[Title/Abstract]) OR ("brain"[Title/Abstract] AND "microvascular abnormalities"[Title/Abstract]) OR ("cerebral"[Title/Abstract] AND "microvascular abnormalities"[Title/Abstract])))

\section{Search strategy for Embase:}

1. cognition/ or attention/ or executive function/ or learning/ or memory/ or socialcognition/

2. cognitive defect/

3. mild cognitive impairment/

4. dementia/

5. Alzheimer disease/

6. 1 or 2 or 3 or 4 or 5

7. “cognition".ti,ab.

8. 6 or 7

9. "cognitive function".ti,ab.

10. 8 or 9

11. "cognitive dysfunction".ti,ab.

12. 10 or 11

13. "cognitive impairment".ti,ab.

14. 12 or 13

15. "neurocognitive".ti,ab.

16. 14 or 15

17. "Mini Mental State Examination".ti,ab.

18. 16 or 17

19. "MMSE".ti,ab.

20. 18 or 19

21. "executive function".ti,ab.

22. 20 or 21

23. "executive functions".ti,ab.

24. 22 or 23

25. "executive functioning".ti,ab.

26. 24 or 25 
27. ("learning" and "memory").ti,ab.

28. 26 or 27

29. "memory impairment".ti,ab.

30. 28 or 29

31. "visual perception".ti,ab.

32. 30 or 31

33. "dementia".ti,ab.

34. 32 or 33

35. "Alzheimer".ti,ab.

36. 34 or 35

37. "Alzheimer's".ti,ab.

38. 36 or 37

39. brain/

40. nuclear magnetic resonance imaging/

41. exp magnetic resonance angiography/

42. neuroimaging/

43. brain infarction/di [Diagnosis]

44. 39 and 40

45. 39 and 41

46. 44 or 45 or 42 or 43

47. ("brain" and "magnetic resonance imaging").ti,ab.

48. 46 or 47

49. ("cerebral" and "magnetic resonance imaging").ti,ab.

50. 48 or 49

51. ("brain" and "MRI").ti,ab.

52. 50 or 51

53. ("cerebral" and "MRI").ti,ab.

54.52 or 53

55. "brain volume".ti,ab.

56. 54 or 55

57. "brain atrophy".ti,ab.

58. 56 or 57

59. "cerebral atrophy".ti,ab.

60. 58 or 59

61. "hippocampal volume".ti,ab.

62. 60 or 61

63. "white matter".ti,ab.

64. 62 or 63

65. ("brain" and "small vessel").ti,ab. 
66. 64 or 65

67. ("cerebral" and "small vessel").ti,ab.

68.66 or 67

69. ("brain" and "microbleeds").ti,ab.

70.68 or 69

71. ("cerebral" and "microbleeds").ti,ab.

72. 70 or 71

73. ("brain" and "infarct").ti,ab.

74. 72 or 73

75. ("brain" and "infarcts").ti,ab.

76. 74 or 75

77. "brain infarction".ti,ab.

78. 76 or 77

79. "cerebral infarct".ti,ab.

80.78 or 79

81. "cerebral infarcts".ti,ab.

82.80 or 81

83. "cerebral infarction".ti,ab.

84.82 or 83

85. "Iacunar".ti,ab.

86.84 or 85

87. ("brain" and "microangiopathy").ti,ab.

88.86 or 87

89. ("cerebral" and "microangiopathy").ti,ab.

90.88 or 89

91. ("brain" and "microvascular abnormalities").ti,ab.

92. 90 or 91

93. "diabetes".ti.

94. "glucose".ti.

95. 93 or 94

96. “insulin”.ti.

97. 95 or 96

98. "glycaemic".ti.

99. 97 or 98

100. "glycemic".ti.

101. 99 or 100

102. "antidiab*".ti.

103. 101 or 102

104. 38 or 92 
105. 104 and 103

106. 105 and 1990:2012.(sa_year).

107. 106 and "Journal: Article".sa_pubt.

\section{Modified Newcastle-Ottawa Quality Assessment Scale}

Risk of bias in studies with cross-sectional and/or longitudinal analyses was assessed by using a modified version of the Newcastle-Ottawa Quality Assessment Scale.

Individual items of the scale are presented below. In the original Newcastle-Ottawa Quality Assessment Scale, selection of both the exposed and non-exposed cohort is rated. The present review included studies that focused on participants with T2DM. Therefore, items 1 and 2 of the Selection category were combined as "representativeness of the diabetic population". Risk of bias was assessed separately for (1) cross-sectional and longitudinal analyses, (2) cognitive and brain MRI outcome measures, and (3) each determinant (ie, HbA1c, fasting glucose levels, fasting insulin levels, HOMA-IR, glucoselowering treatment, and hypoglycaemic events).

For cross-sectional studies, items S1, S2, C1, and $\mathrm{O} 1$ are rated. For longitudinal studies, all items are rated. A study can be awarded a maximum of one star for each numbered item within the Selection and Outcome categories. A maximum of two stars can be given for Comparability.

We considered cross-sectional studies that received 4 or 5 points to be at low risk of bias, studies that scored 3 points to be at moderate risk, and those with less than 3 points to be at high risk of bias. Longitudinal studies were at low risk of bias if their score was above 6 , at moderate risk if they scored 5 or 6 points, and at high risk of bias with scores below 5 .

\section{Selection}

1. Representativeness of the diabetic (T2DM) population (based on age, sex, diabetes duration, glucose-lowering treatment, complications, and comorbidities)

a) Truly representative of the general diabetic (T2DM) population *

b) Somewhat representative of the general diabetic (T2DM) population *

c) Selected group of participants with T2DM

d) No description of the derivation of the population

2. Ascertainment of determinants

a) For glucose-related and insulin-related variables: based on venous blood sampling * 
For glucose-lowering treatment: based on medical/ pharmaceutical records * For hypoglycaemic events: based on medical records *

b) For glucose-related and insulin-related variables: based on peripheral blood sampling* For glucose-lowering treatment: medication containers brought to the clinic visit or self-reported*

For hypoglycaemic events: self-reported *

c) For glucose-related and insulin-related variables: based on medical records

d) No description

3. Demonstration that outcome of interest was not present at start of study (ie, cognitive function was better, or brain MRI abnormalities were not, or to a lesser extent, present at start of the study)

a) Yes*

b) No

\section{Comparability}

1. Comparability of cohorts on the basis of the design or analysis

a) Analysis controls for age *

b) Analysis controls for sex (and educational level [when reporting on cognitive outcome measures])* if the study population consisted of only male or female participants, a star was awarded

\section{Outcome}

1. Assessment of outcome

a) For cognitive outcome measures: based on well-validated cognitive tests* For brain MRI abnormalities: based on a 1.5 Tesla MRI scan with the following minimum essential sequences: for brain atrophy (T1-weighted), for WML (T2weighted and/or FLAIR), for (silent) cerebral infarcts (T1-weighted and/or T2weighted), for cerebral microbleeds (T2*-weighted gradient-recalled echo) *

b) For cognitive outcome measures (ie,): based on ICD codes*

c) Otherwise than described above

d) No description

2. Was follow-up long enough for outcomes to occur

a) Yes (follow-up period of at least 3 years) *

b) No

3. Adequacy of follow up of cohorts

a) Complete follow up - all subjects accounted for * 
b) Subjects lost to follow up unlikely to introduce bias ( $>80 \%$ follow up, or description provided of those lost) *

c) Follow up rate $<80 \%$ and no description of those lost

d) No statement

\section{Abbreviations}

T2DM = type 2 diabetes mellitus; HOMA-IR = Homeostasis Model Assessment of insulin resistance; $\mathrm{MRI}=$ Magnetic Resonance Imaging; FLAIR = Fluid Attenuated Inversion Recovery 


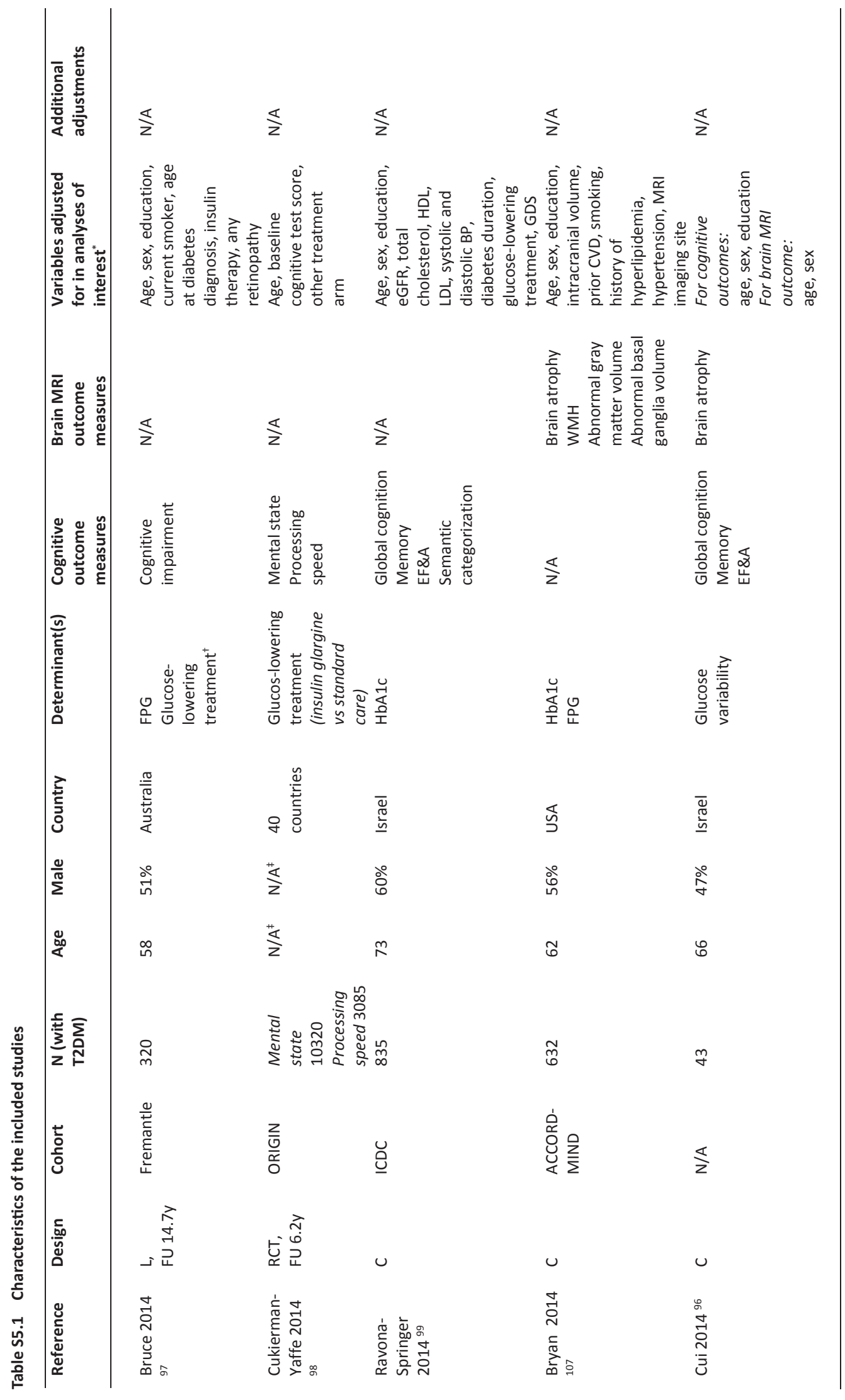




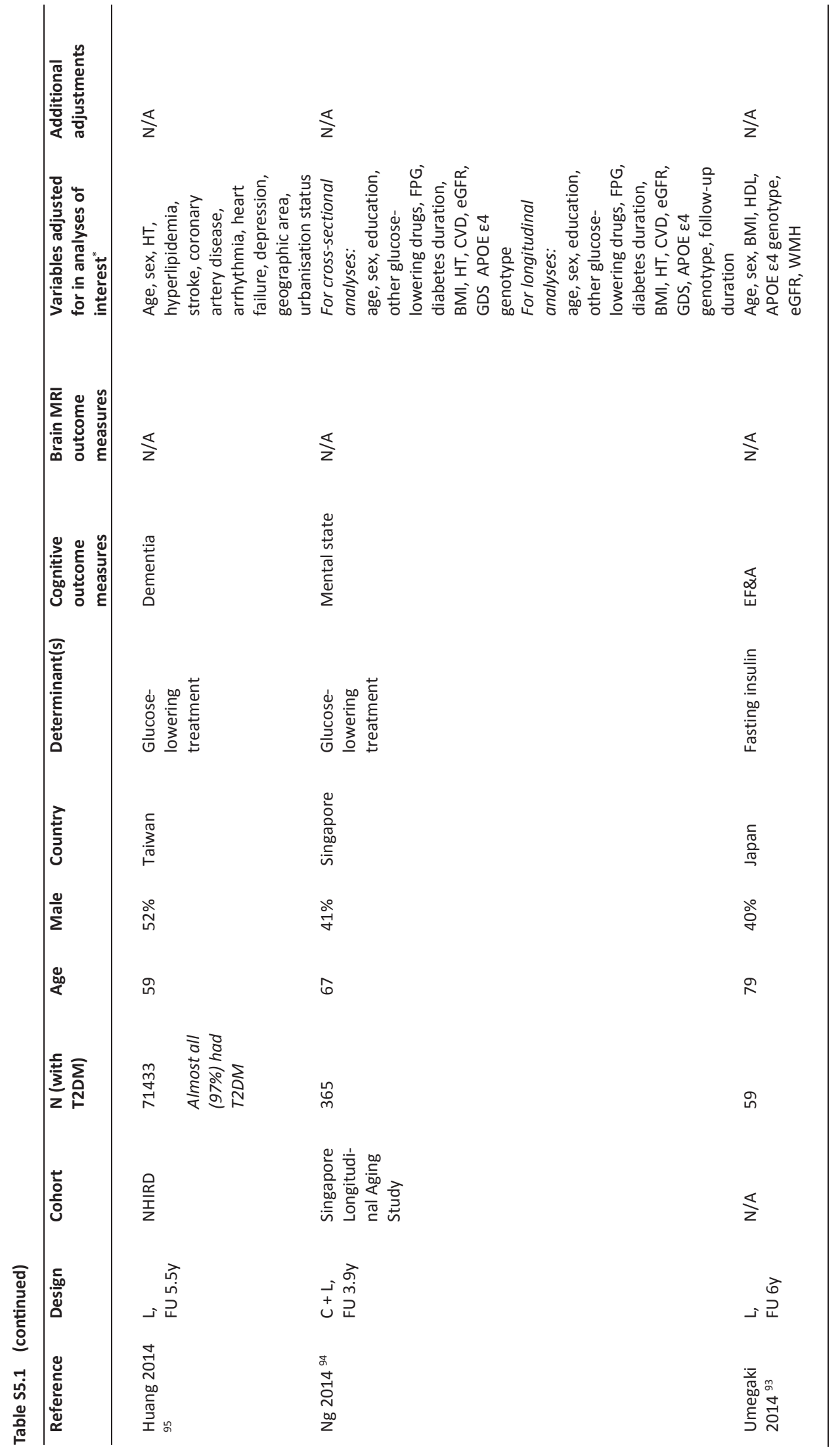




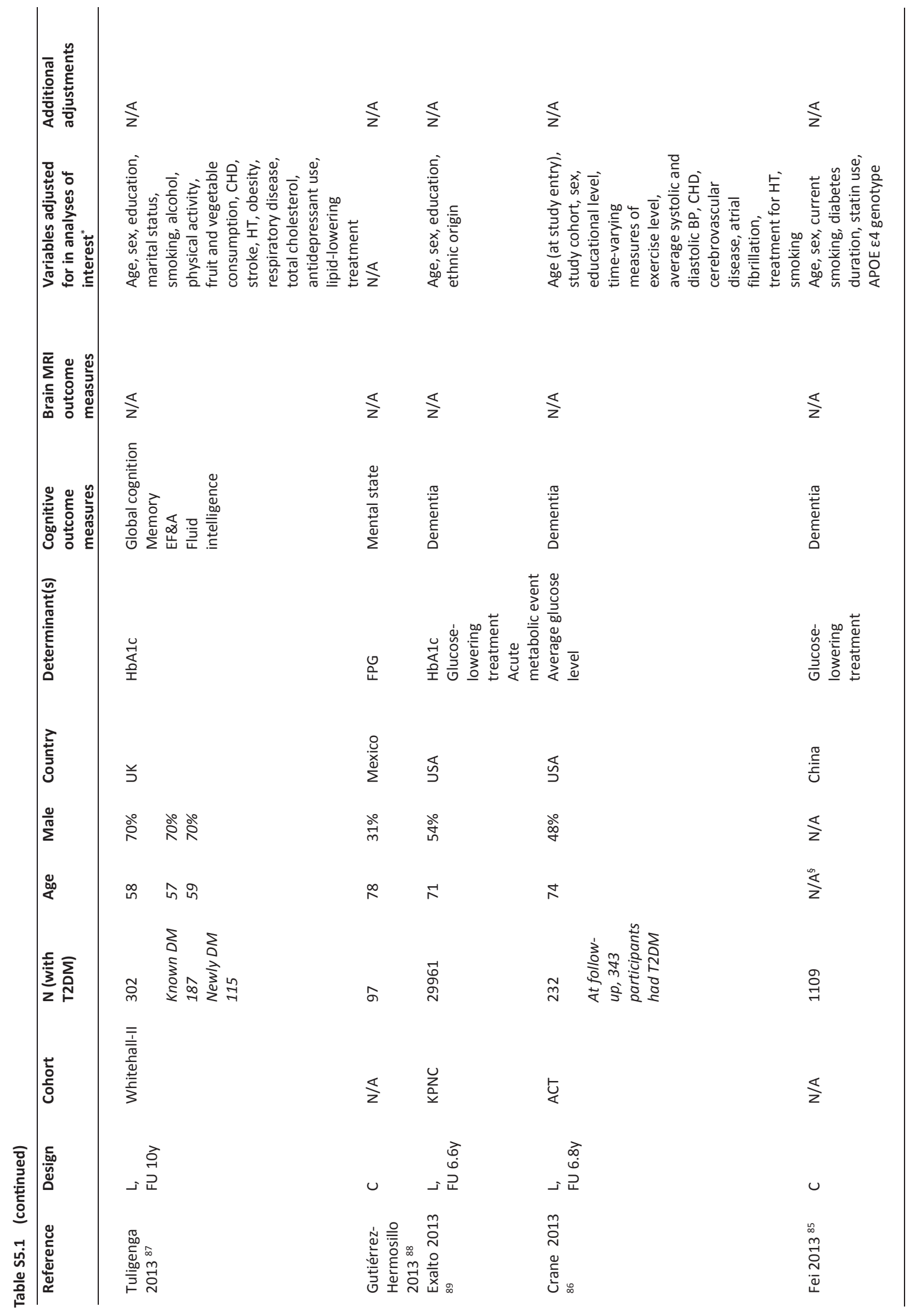




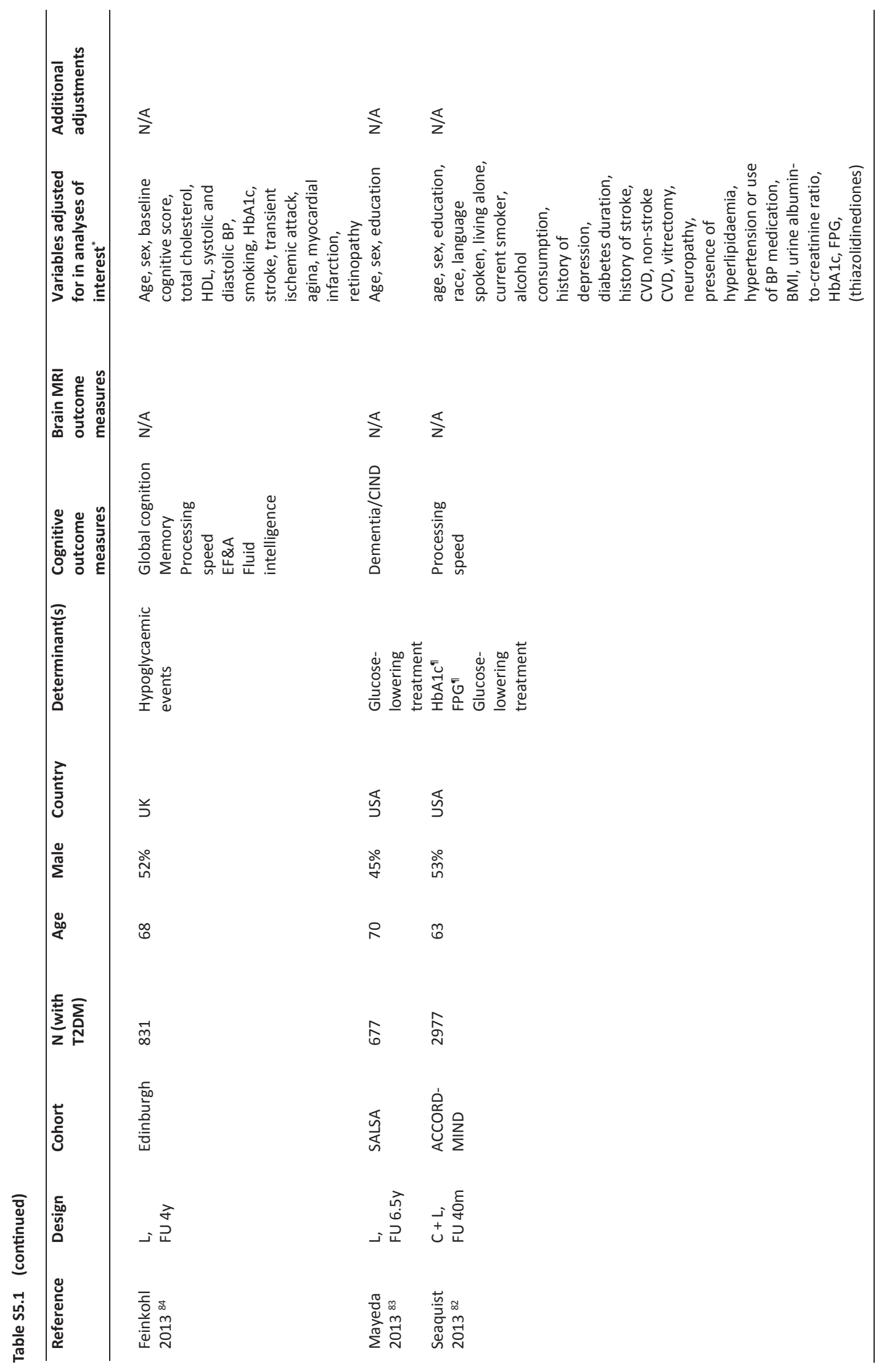




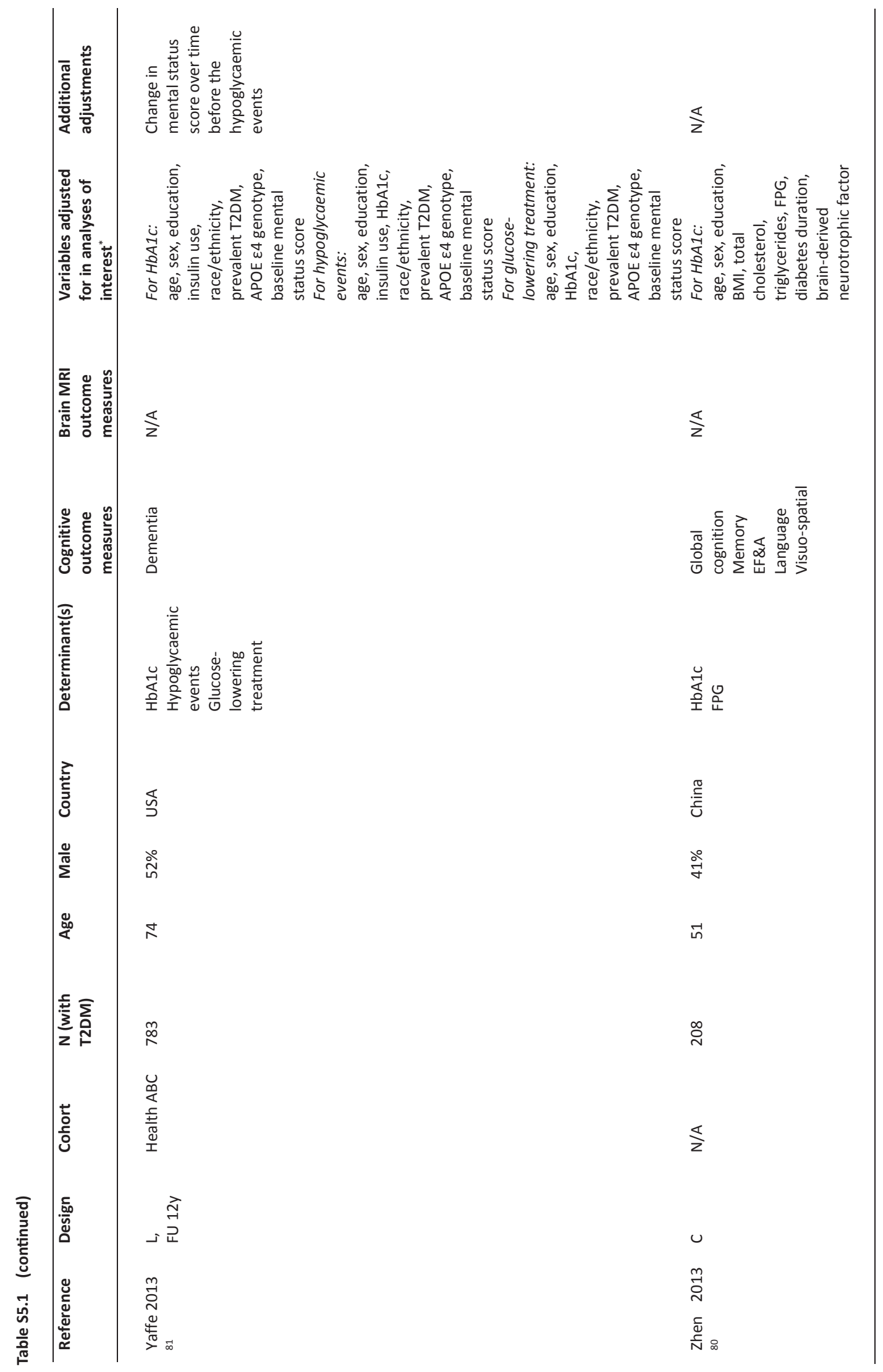




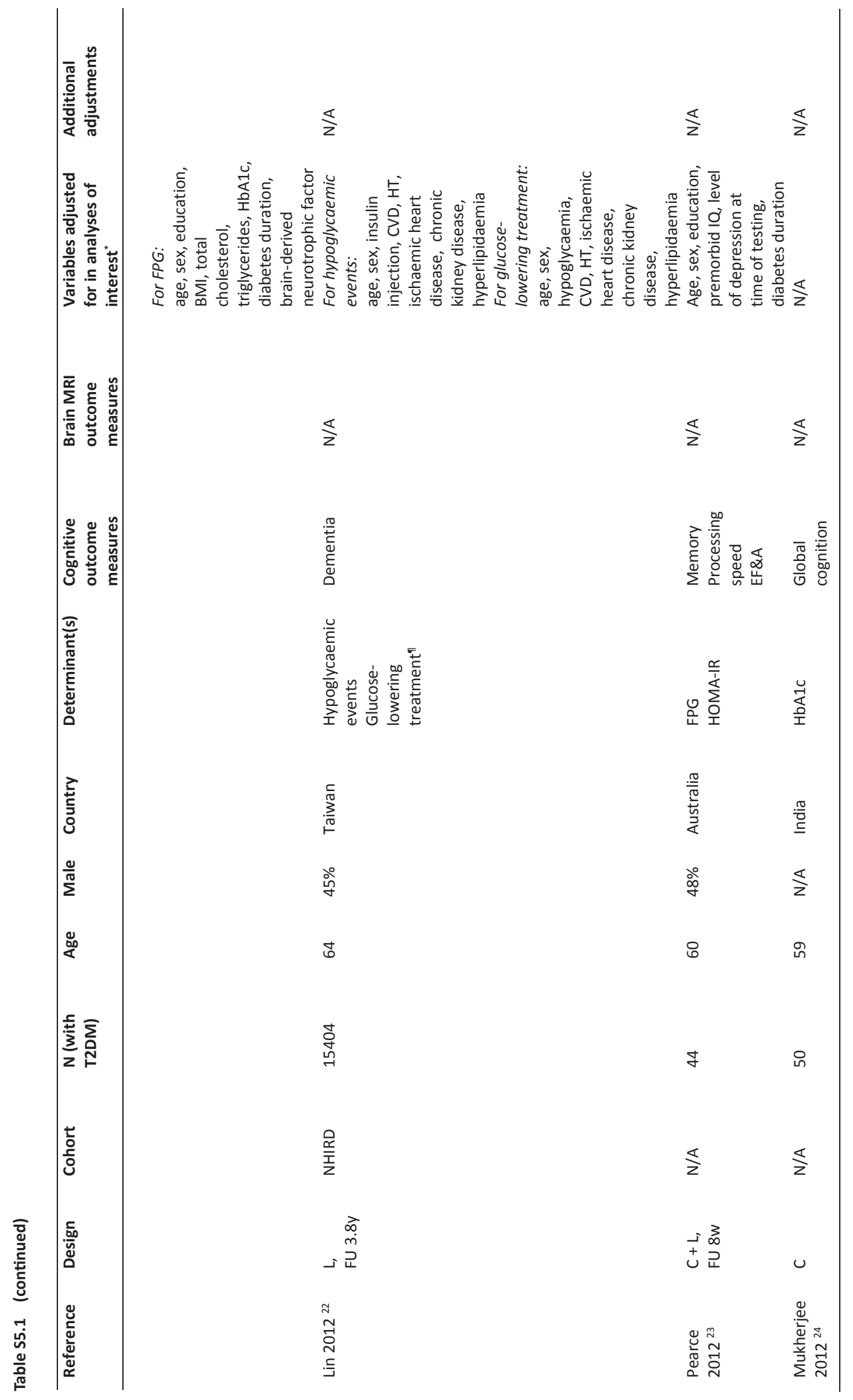




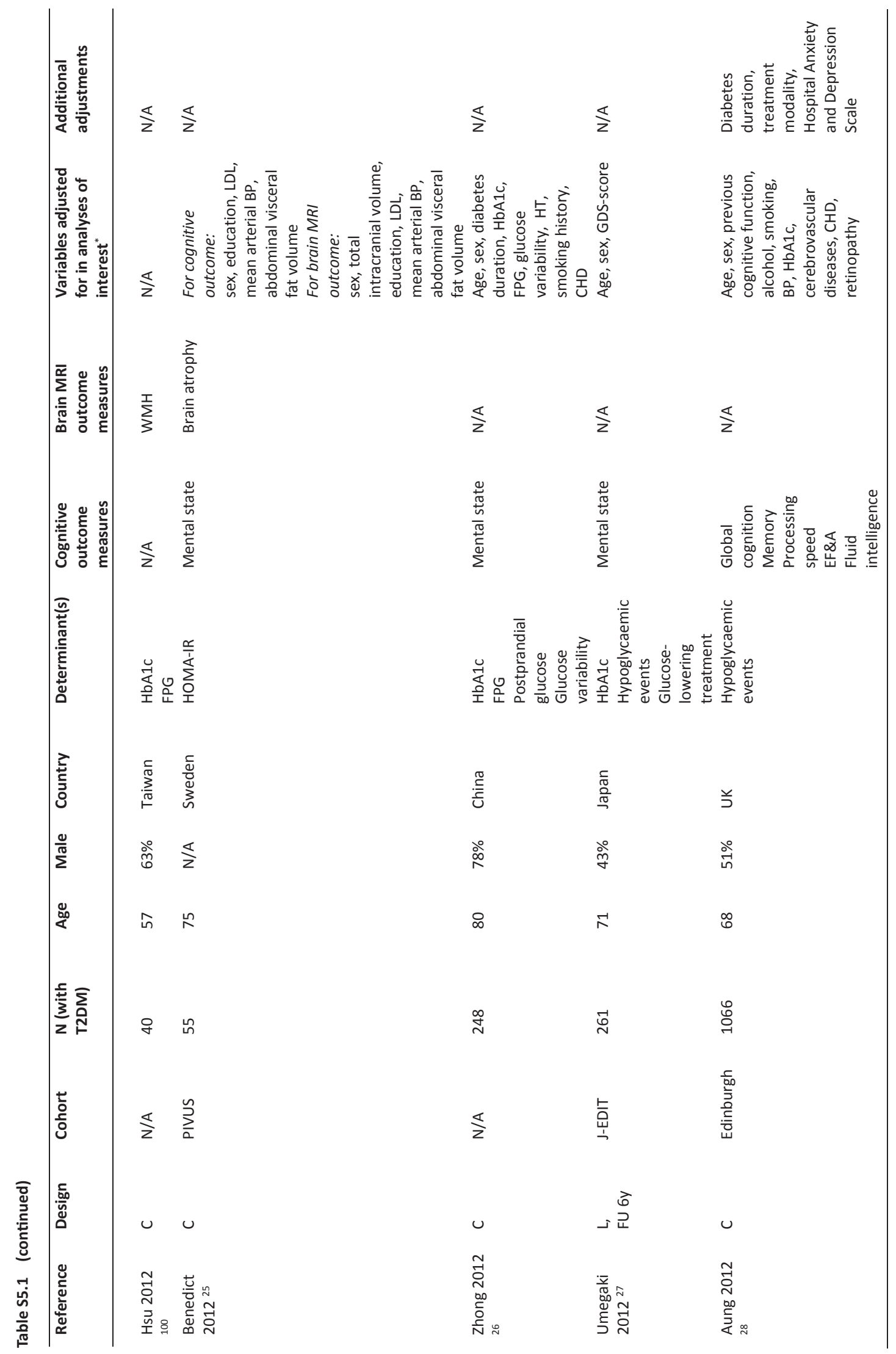




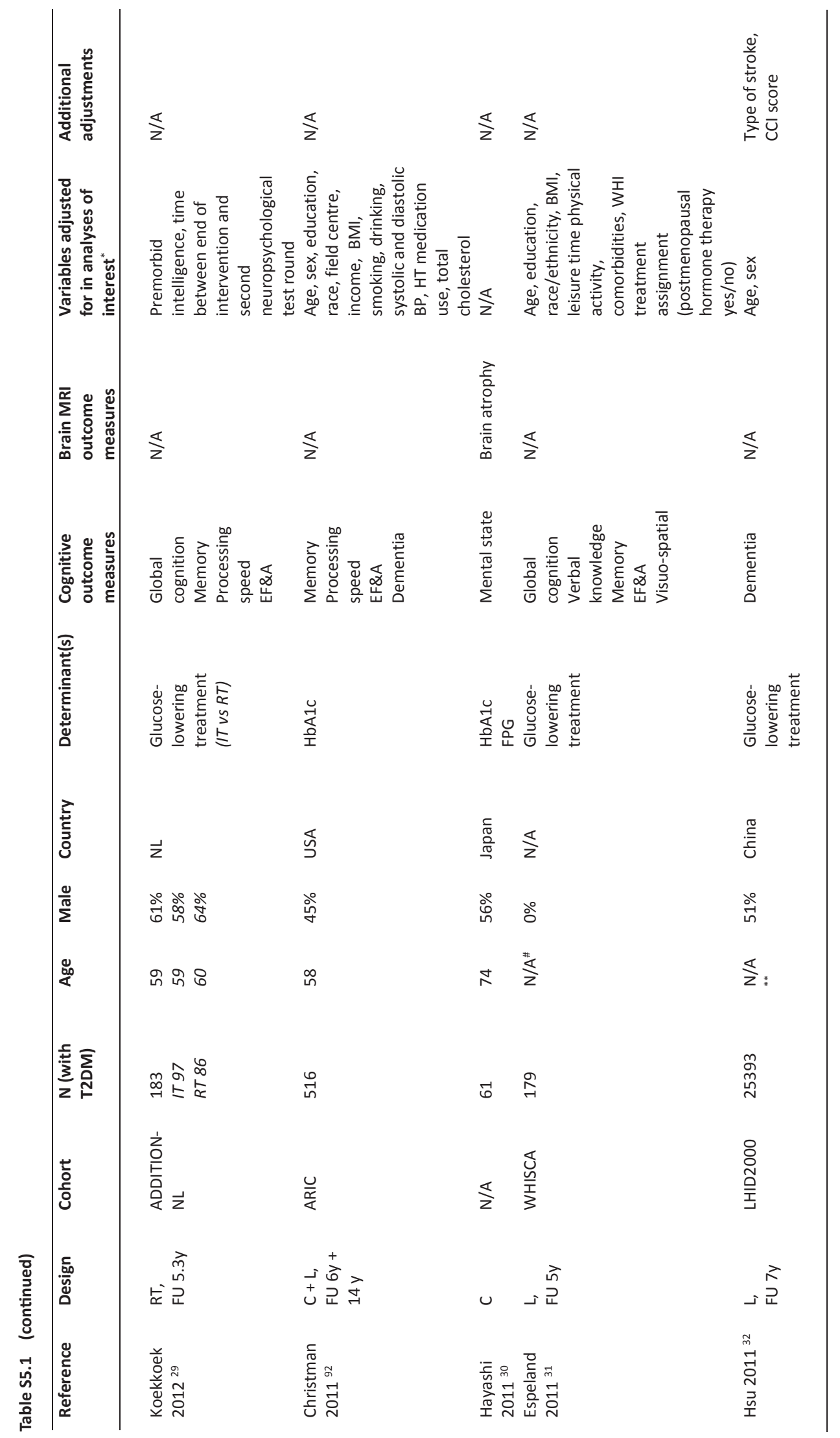




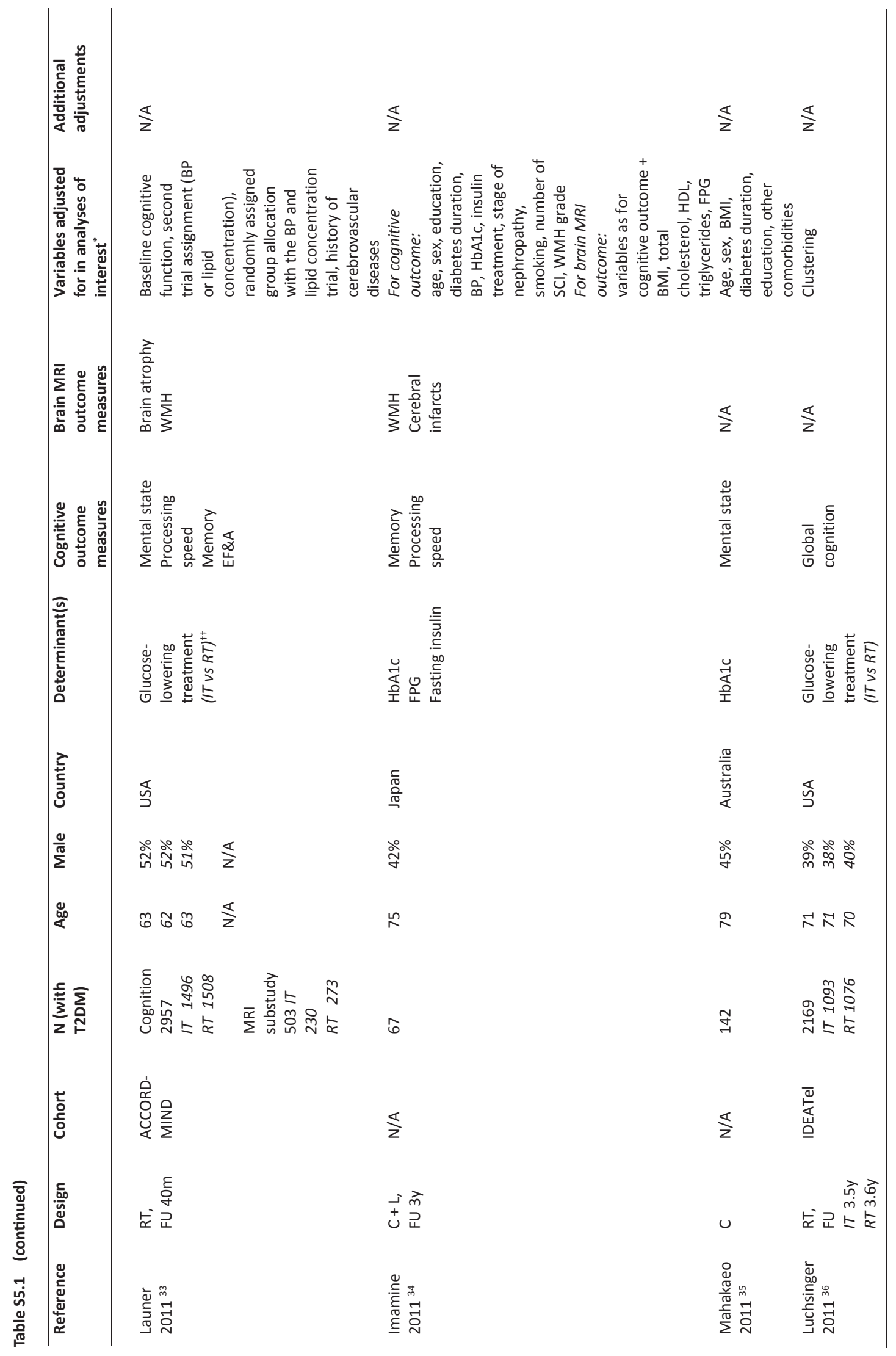




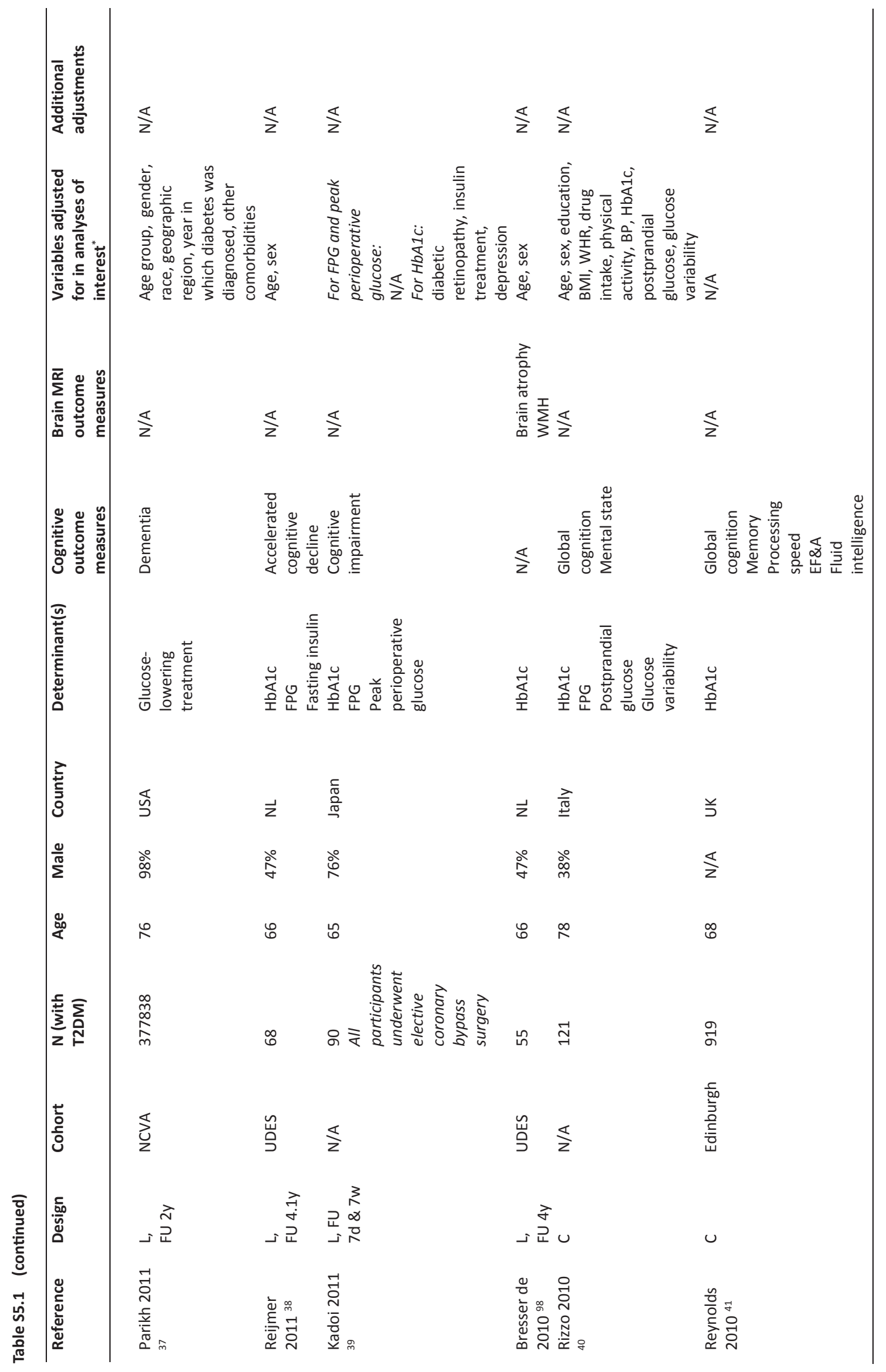




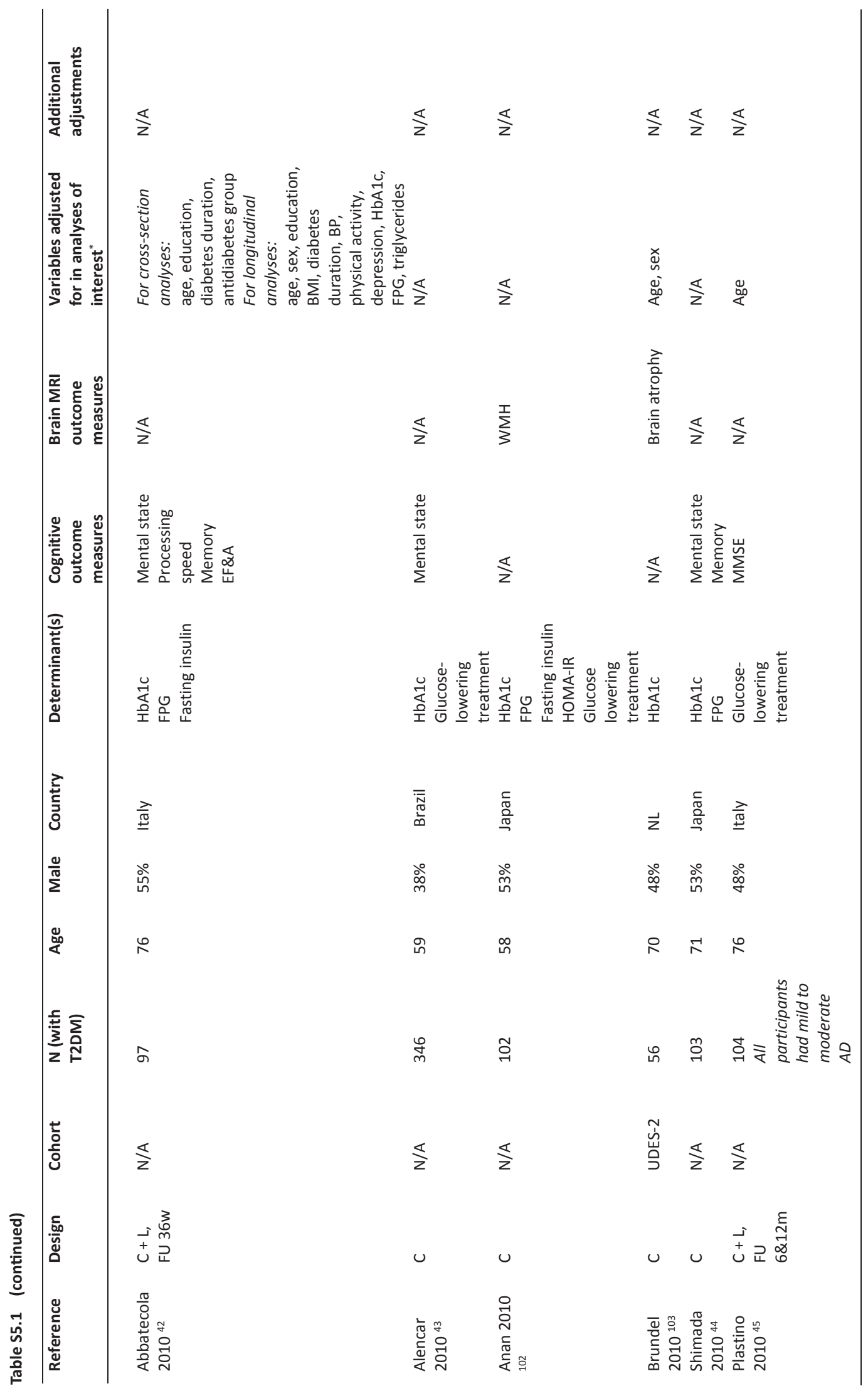




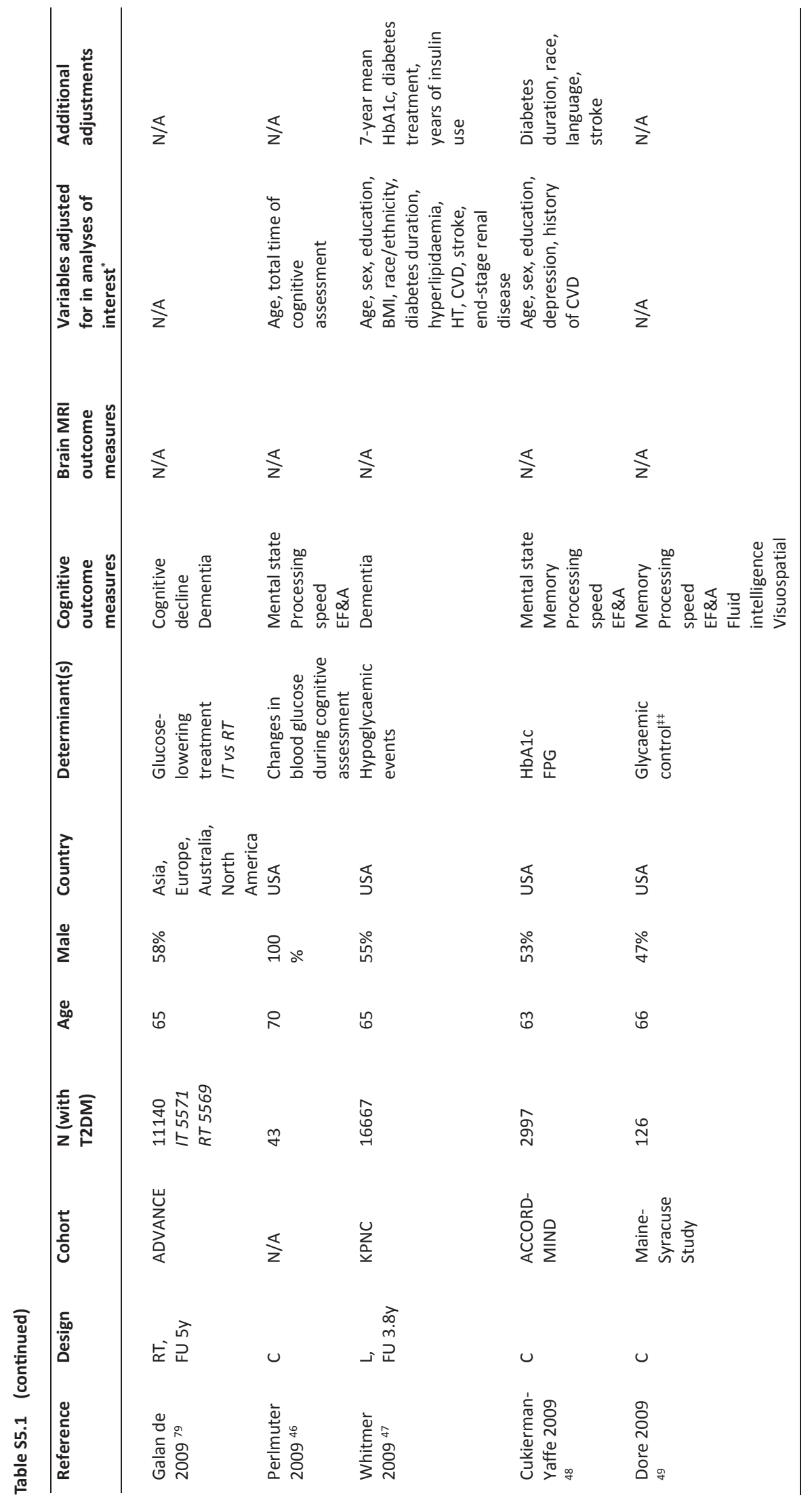


$\frac{\pi}{2} \quad \frac{\pi}{z} \quad \frac{\pi}{z} \quad \frac{\pi}{z}$

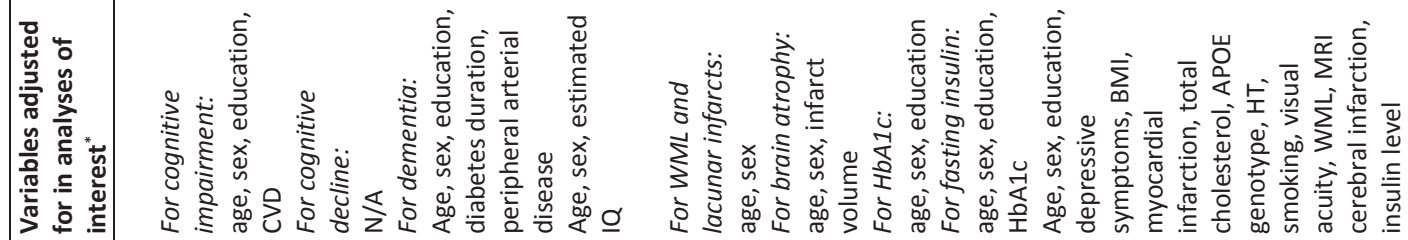

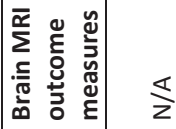

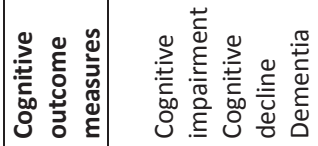

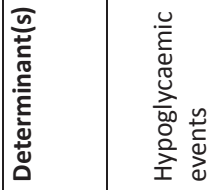

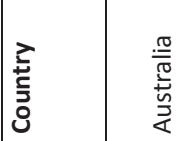

एँ.

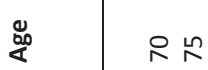

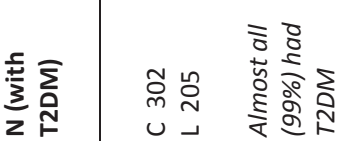

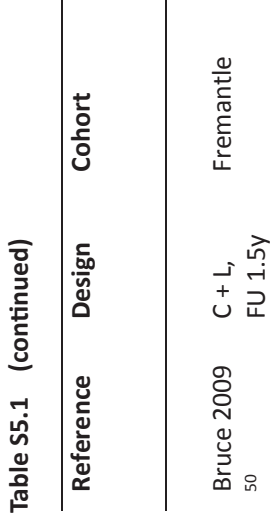

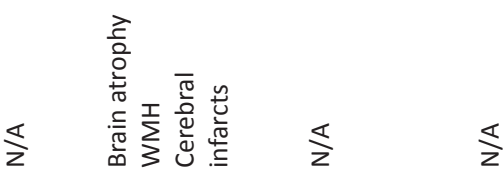

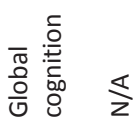

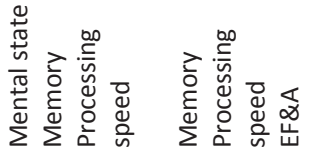

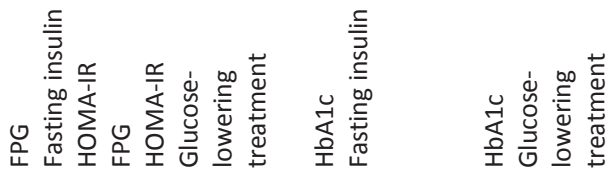

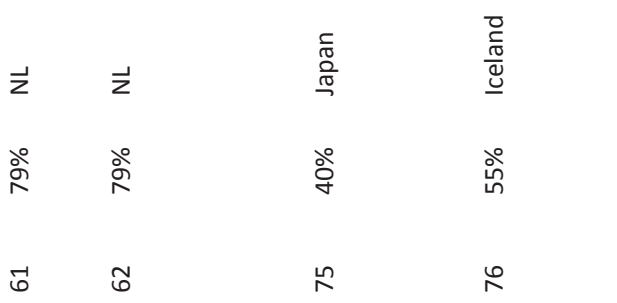

유 롬

$\curvearrowright$

$\stackrel{\infty}{\sim}$

$\sum_{\text {in }}^{\frac{c}{c}} \sum_{n}^{\frac{1}{c}}$

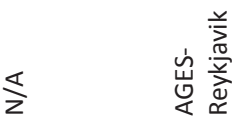

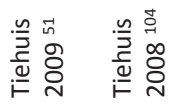

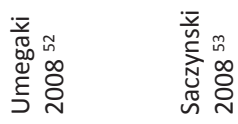




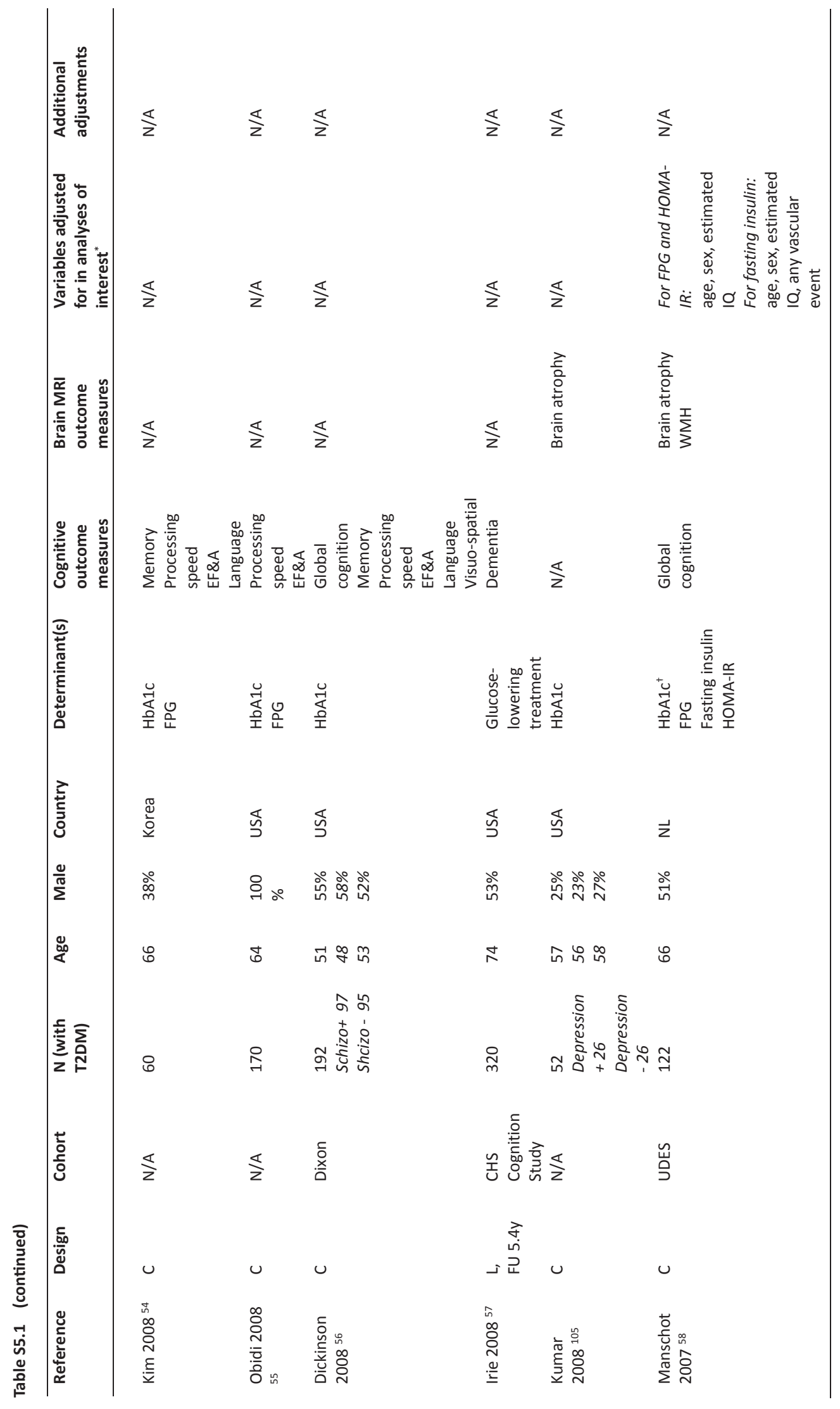




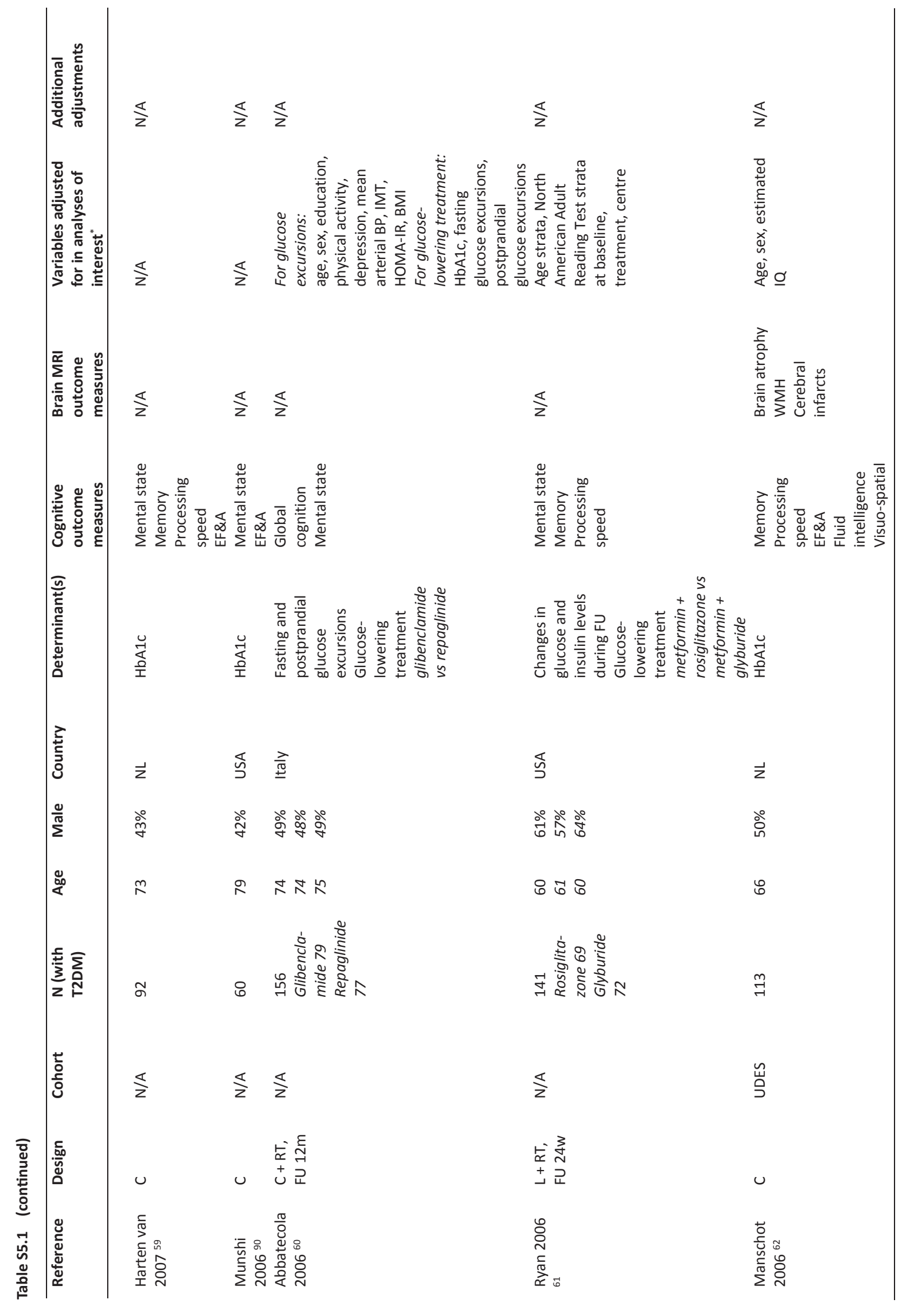




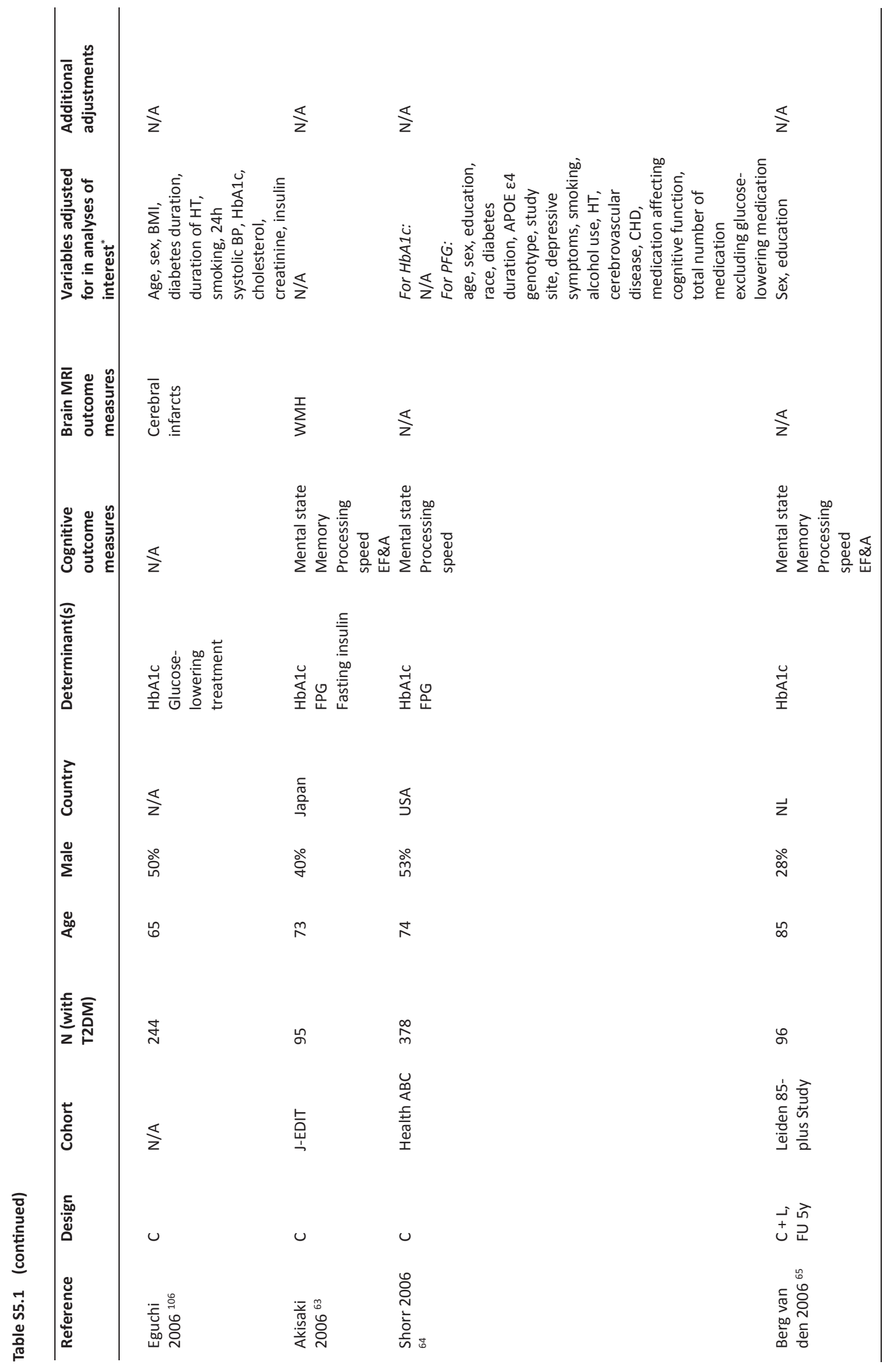




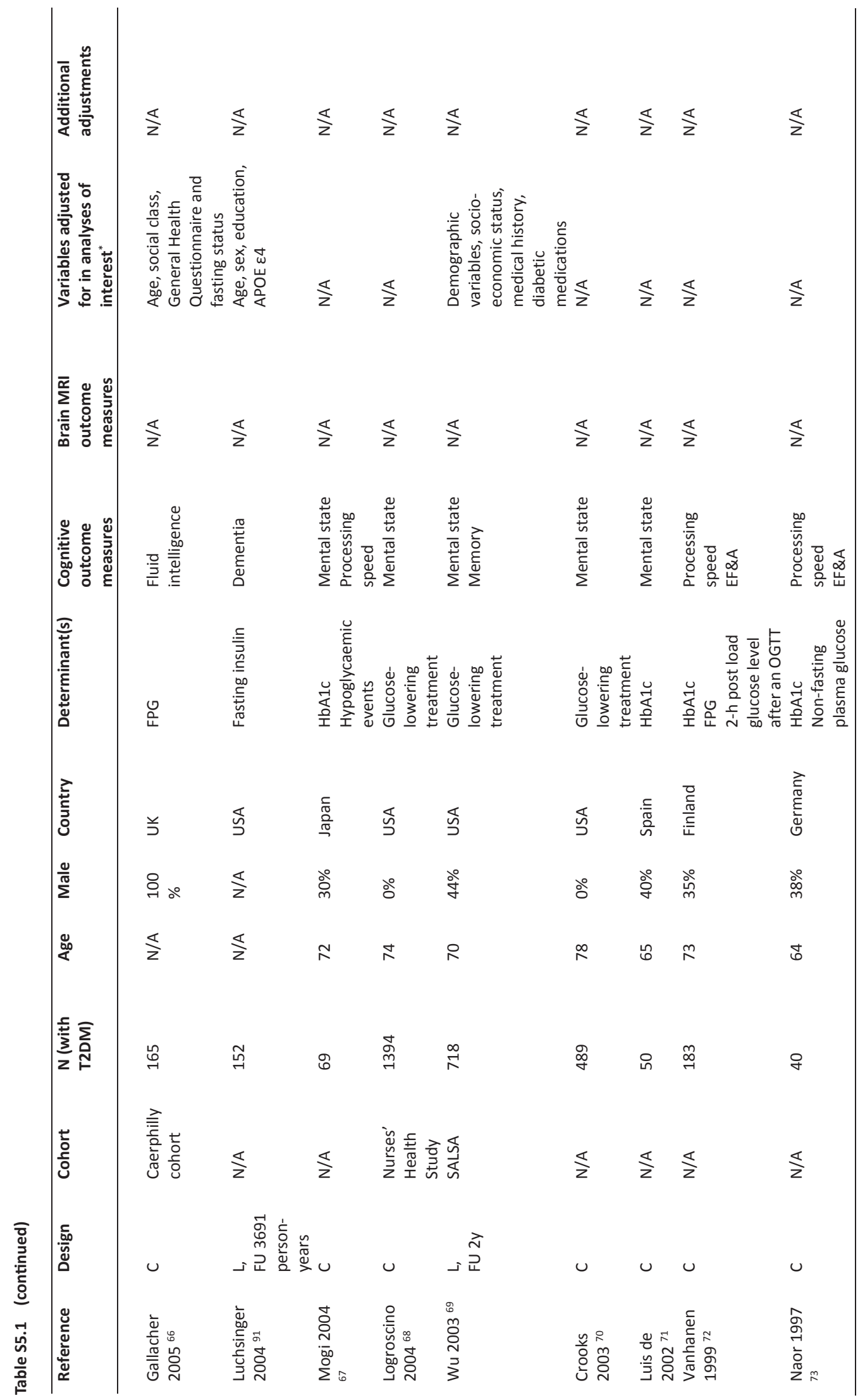




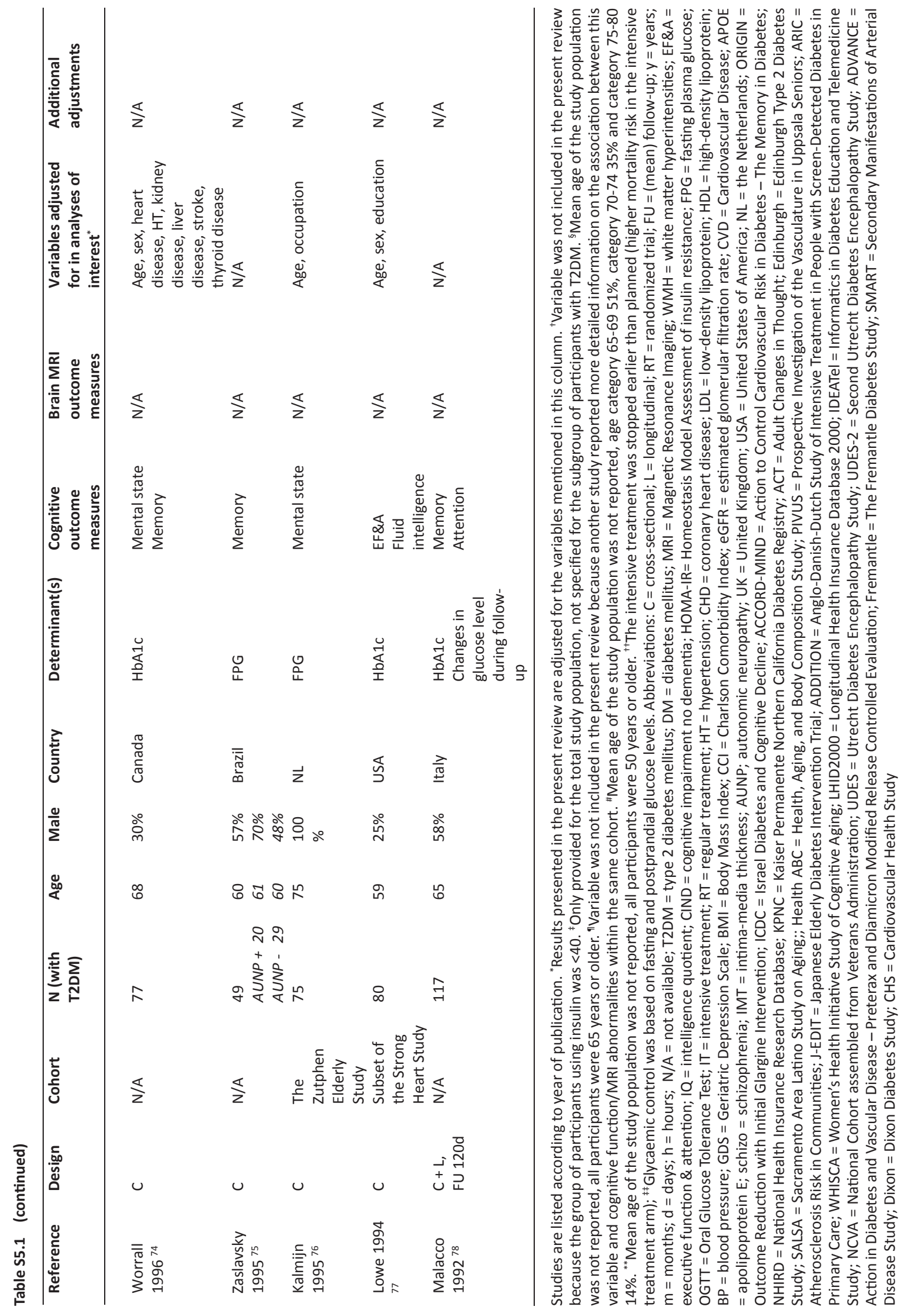


Table S5.2 Classification of neuropsychological tests into cognitive domains*

\begin{tabular}{|c|c|}
\hline Cognitive domain & Cognitive tests \\
\hline \multirow[t]{8}{*}{ Mental state } & Mini-Mental State Examination \\
\hline & Modified Mini Mental State Examination (3MS/7MS) \\
\hline & Cambridge Cognitive Examination \\
\hline & Cambridge Neuropsychological Test Automated Battery \\
\hline & Hiv Dementia Scale \\
\hline & Revised Hasegawa's Dementia Scale \\
\hline & Clock Drawing Test \\
\hline & Modified Telephone Interview for Cognitive Status \\
\hline \multirow[t]{19}{*}{ Memory } & Rey's Auditory Verbal Learning Test \\
\hline & Simplified Rey Figure Test \\
\hline & Logical Memory Test (Wechsler Memory Scale) \\
\hline & Faces and Family Picture Test ( Wechsler Memory Scale ) \\
\hline & Digit Span Forward (Wechsler Adult Intelligence Scale) \\
\hline & Elderly Verbal Learning Test \\
\hline & Word recall (Alzheimer's Disease Assessment Scale) \\
\hline & Delayed Word Recall Test \\
\hline & Famous Faces Test \\
\hline & Hopkins Verbal Learning Test \\
\hline & The Visual Reproduction Test \\
\hline & Paired Associates Learning \\
\hline & 12 Word Learning Test \\
\hline & Rivermead Behavioural Memory Test \\
\hline & Paragraph recall (Neuropsychological test from \\
\hline & National Centre of Neurology and Psychiatry, Japan) \\
\hline & List, story and figure learning (Repeatable Battery for \\
\hline & the Assessment of Neuropsychological Status) \\
\hline & Recognition of tower silhouettes \\
\hline \multirow[t]{8}{*}{ Processing speed } & Digit Symbol Substitution Test (Wechsler Adult Intelligence Scale) \\
\hline & Trail Making Test Part A \\
\hline & Stroop Color Word Test Part I and II \\
\hline & Choice Reaction Time \\
\hline & (Simple) Reaction Time Test \\
\hline & Inspection Time \\
\hline & Aufmerksamkeits-Belasting-Test d2 (speed component) \\
\hline & Bourdon-Freyberg-concentration test (speed component) \\
\hline \multirow[t]{9}{*}{ Executive function \& attention } & Digit Span Backwards (Wechsler Adult Intelligence Scale) \\
\hline & Letter Number Sequencing (Wechsler Adult Intelligence Scale) \\
\hline & Trail Making Test Part B \\
\hline & Stroop Color Word Test Part III \\
\hline & Verbal Fluency (in some studies specified as \\
\hline & Controlled Oral Word Association Test) \\
\hline & Clock-in-a-box test \\
\hline & Aufmerksamkeits-Belasting-Test d2 (number of errors) \\
\hline & Bourdon-Freyberg-concentration test (number of errors) \\
\hline \multirow[t]{3}{*}{ Fluid intelligence } & Matrix Reasoning (Wechsler Adult Intelligence Scale) \\
\hline & Similarities (Wechsler Adult Intelligence Scale) \\
\hline & Alice Heim 4 (part 1) \\
\hline Language & A short form of the Korean-Boston Naming Test \\
\hline \multirow[t]{5}{*}{ Visuospatial abilities } & Block Design (Wechsler Adult Intelligence Scale) \\
\hline & Object Assembly (Wechsler Adult Intelligence Scale) \\
\hline & Hooper Visual Organization Test \\
\hline & Judgment of Line Orientation Test \\
\hline & Card Rotations Test \\
\hline
\end{tabular}

${ }^{*}$ Cognitive tests were only classified into domains when this was not done in the original paper. Otherwise, cognitive domains were based on the classifications used in the original papers. 


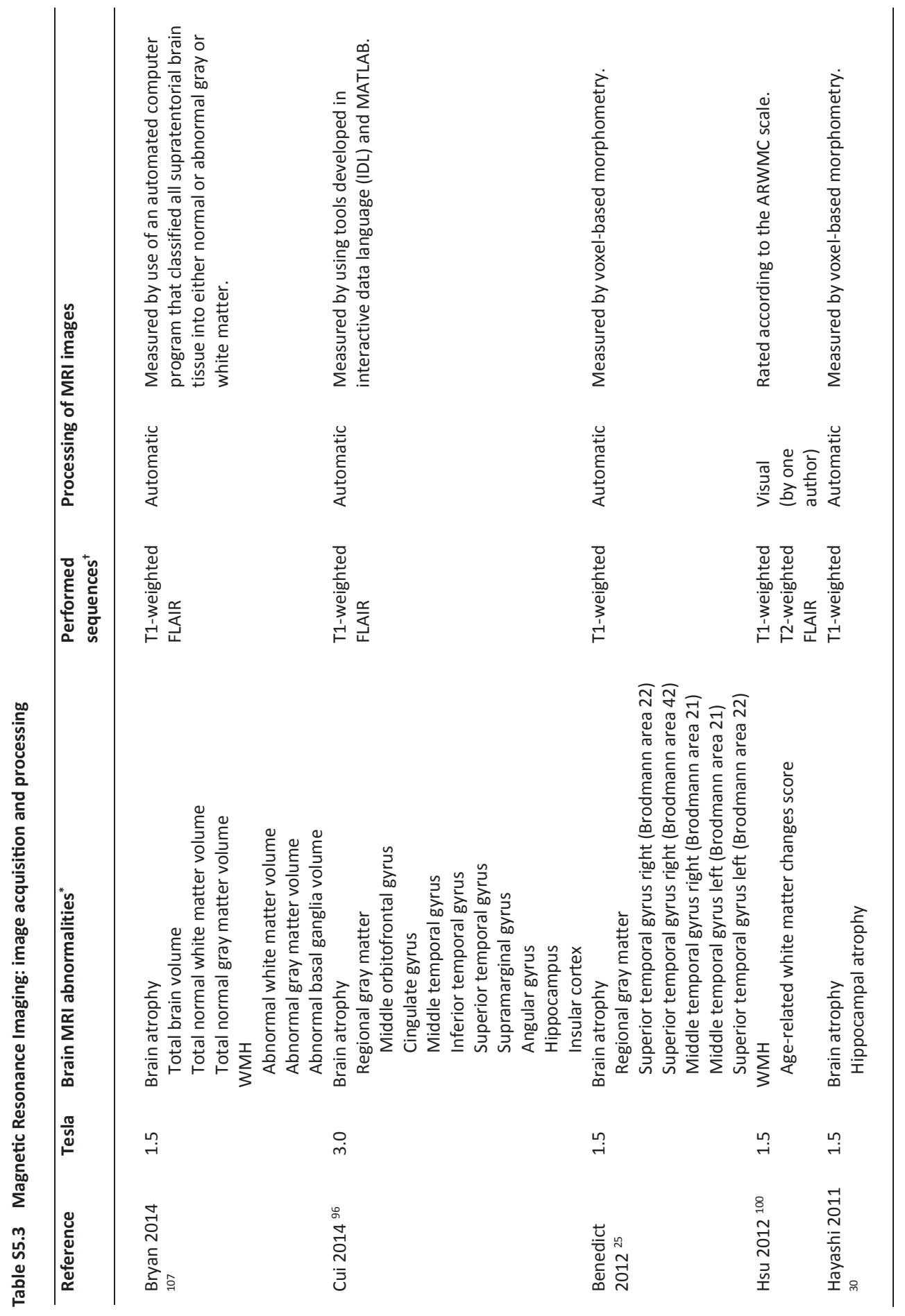


Type 2 diabetes, cognition, and brain MRI

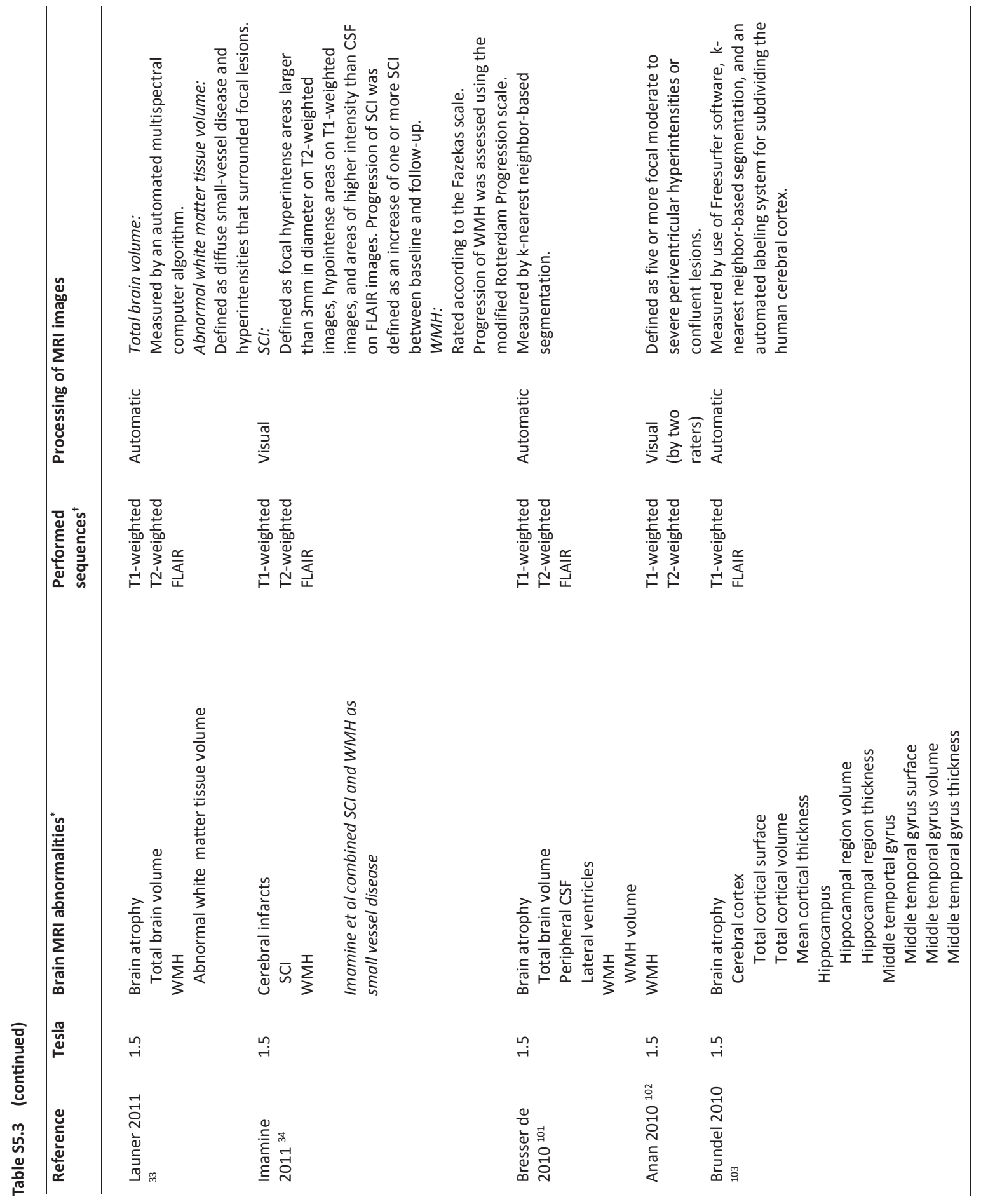




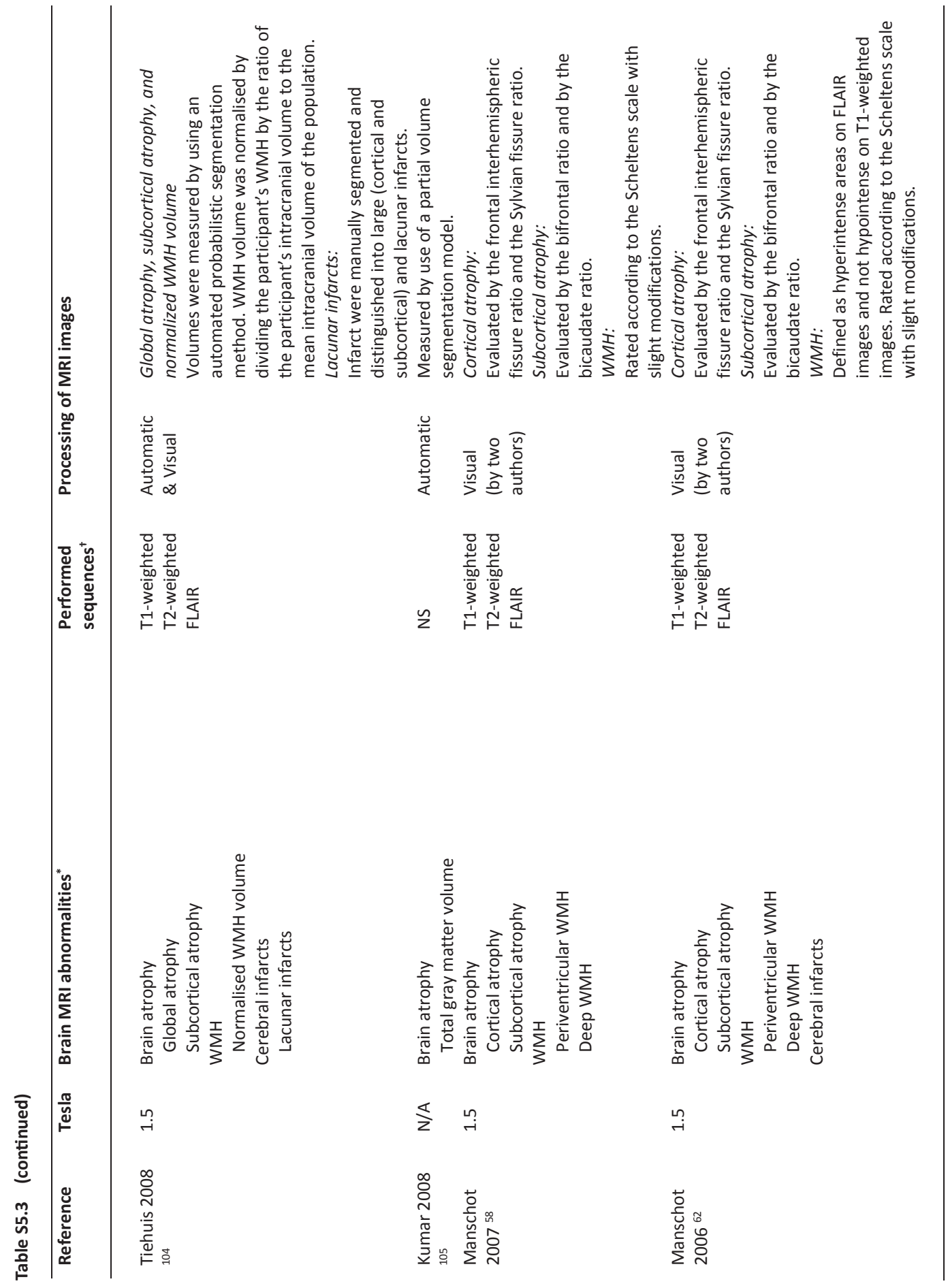


Type 2 diabetes, cognition, and brain MRI

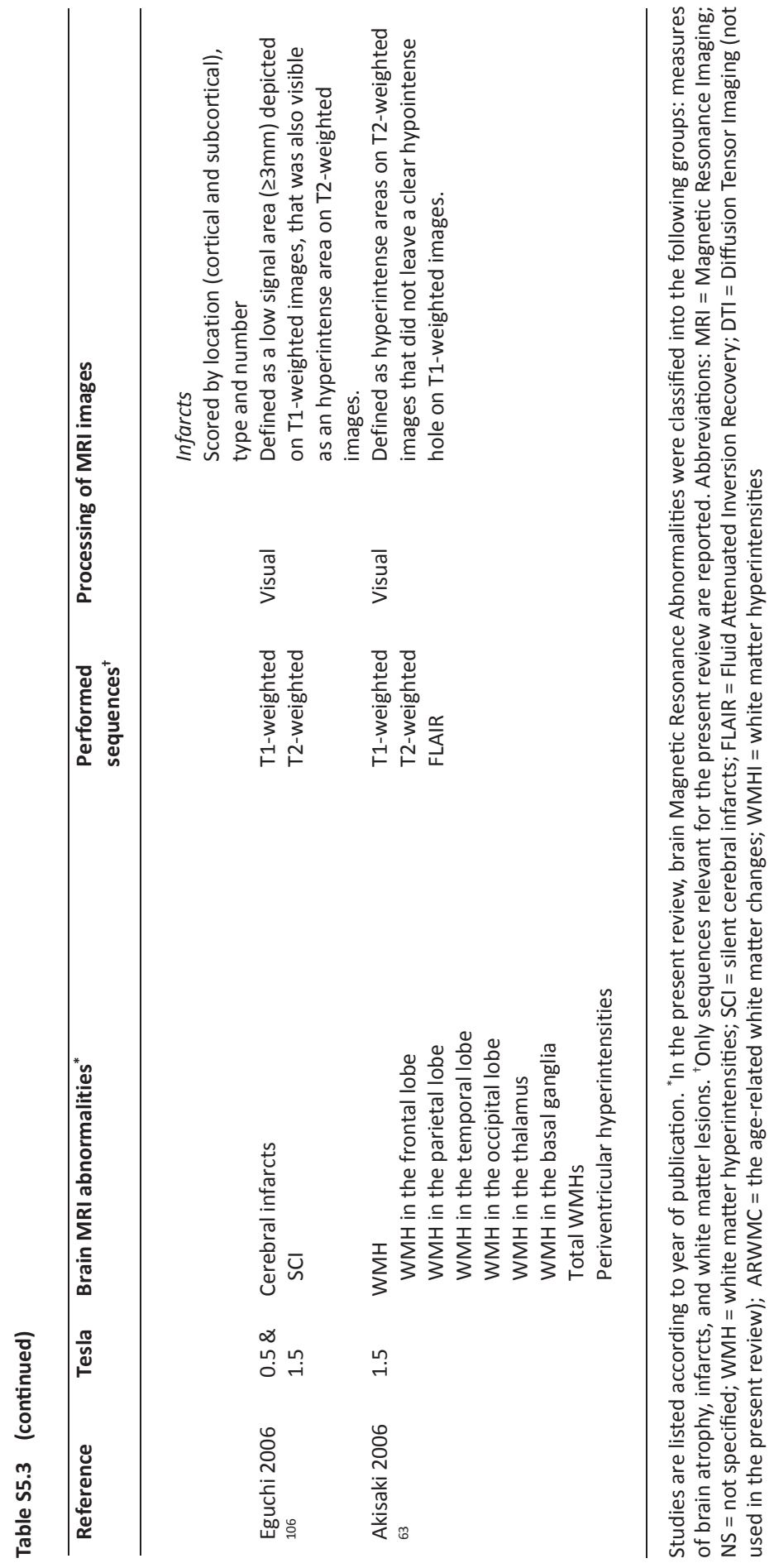


Table S5.4 Risk of bias for studies with cross-sectional and/or longitudinal study-design

\begin{tabular}{lllllllll}
\hline Reference & S1 & S2 & S3 $^{*}$ & C1 & 01 & O2 $^{*}$ & O3 $^{*}$ & $\begin{array}{c}\text { Total score } \\
\text { (max) }\end{array}$
\end{tabular}

$(\max )$

HbA1c \& cognitive function

Ravona-Springer 201499

Tuligenga $2013^{87}$

Exalto $2013^{89}$

Yaffe $2013^{81}$

Zhen $2013^{80}$

Mukherjee $2012{ }^{24}$

Zhong $2012^{26}$

Umegaki $20122^{27}$

Hayashi $2011^{30}$

Christman 201192

Christman $2011^{92}$

Imamine $2011^{34}$

Mahakaeo $2011^{35}$

Reijmer $2011^{38}$

Kadoi $2011^{39}$

Rizzo $2010^{40}$

Reynolds $2010^{41}$

Abbatecola $2010^{42}$

Alencar $2010^{43}$

Shimada $2010^{44}$

Cukierman-Yaffe 200948

Umegaki $2008^{52}$

Saczynski $2008^{53}$

Kim $2008^{54}$

Obidi $2008^{55}$

Dickinson $2008^{56}$

Harten van $2007^{59}$

Manschot $2006^{62}$

Munshi $2006^{90}$

Akisaki $2006^{63}$

Shorr $2006^{64}$

Berg van den $2006^{65}$

Berg van den $2006^{65}$

Mogi 200467

Luis de $2002^{71}$

Vanhanen $1999^{72}$

Naor $1997^{73}$

Worrall $1996^{74}$

Lowe 199477

Malacco $1992^{78}$

$\begin{array}{cccccccc}1 & 0 & \ldots & 2 & 1 & \ldots & \ldots & 4(5) \\ 1 & 1 & 1 & 2 & 1 & 1 & 1 & 8(8) \\ 1 & 1 & 1 & 2 & 1 & 1 & 1 & 8(8) \\ 1 & 1 & 1 & 2 & 1 & 1 & 1 & 8(8) \\ 0 & 1 & \ldots & 2 & 1 & \ldots & \ldots & 4(5) \\ 0 & 0 & \ldots & 0 & 1 & \ldots & \ldots & 1(5) \\ 1 & 1 & \ldots & 1 & 1 & \ldots & \ldots & 4(5) \\ 1 & 1 & 1 & 1 & 1 & 1 & 1 & 7(8) \\ 0 & 0 & \ldots & 0 & 1 & \ldots & \ldots & 1(5) \\ 1 & 1 & \ldots & 2 & 1 & \ldots & \ldots & 5(5) \\ 1 & 1 & 1 & 2 & 1 & 1 & 1 & 8(8) \\ 1 & 1 & \ldots & 2 & 1 & \ldots & \ldots & 5(5) \\ 1 & 1 & \ldots & 2 & 1 & \ldots & \ldots & 5(5) \\ 1 & 1 & 1 & 1 & 1 & 1 & 1 & 7(8) \\ 0 & 1 & 1 & 0 & 1 & 0 & 1 & 4(8) \\ 0 & 1 & \ldots & 2 & 1 & \ldots & \ldots & 4(5) \\ 1 & 1 & \ldots & 0 & 1 & \ldots & \ldots & 3(5) \\ 0 & 1 & \ldots & 1 & 1 & \ldots & \ldots & 3(5) \\ 1 & 1 & \ldots & 0 & 1 & \ldots & \ldots & 3(5) \\ 0 & 1 & \ldots & 0 & 1 & \ldots & \ldots & 2(5) \\ 0 & 1 & \ldots & 2 & 1 & \ldots & \ldots & 4(5) \\ 1 & 1 & \ldots & 2 & 1 & \ldots & \ldots & 5(5) \\ 0 & 1 & \ldots & 2 & 1 & \ldots & \ldots & 4(5) \\ 1 & 1 & \ldots & 0 & 1 & \ldots & \ldots & 3(5) \\ 0 & 0 & \ldots & 0 & 1 & \ldots & \ldots & 1(5) \\ 0 & 0 & \ldots & 0 & 1 & \ldots & \ldots & 1(5) \\ 0 & 1 & \ldots & 0 & 1 & \ldots & \ldots & 2(5) \\ 1 & 1 & \ldots & 2 & 1 & \ldots & \ldots & 5(5) \\ 1 & 0 & \ldots & 0 & 1 & \ldots & \ldots & 2(5) \\ 1 & 1 & \ldots & 0 & 1 & \ldots & \ldots & 3(5) \\ 1 & 1 & \ldots & 0 & 1 & \ldots & \ldots & 3(5) \\ 0 & 1 & \ldots & 2 & 1 & \ldots & \ldots & 4(5) \\ 0 & 1 & 1 & 2 & 1 & 1 & 1 & 7(8) \\ 1 & 1 & \ldots & 0 & 1 & \ldots & \ldots & 3(5) \\ 1 & 1 & \ldots & 0 & 1 & \ldots & \ldots & 3(5) \\ 0 & 1 & \ldots & 0 & 1 & \ldots & \ldots & 2(5) \\ 0 & 1 & \ldots & 0 & 1 & \ldots & \ldots & 2(5) \\ 1 & 1 & \ldots & 1 & 1 & \ldots & \ldots & 4(5) \\ 0 & 1 & \ldots & 2 & 1 & \ldots & \ldots & 4(5) \\ 0 & 1 & \ldots & 0 & 1 & \ldots & \ldots & 2(5)\end{array}$

Fasting glucose levels \& cognitive function

\begin{tabular}{lllllllll} 
Bruce $2014^{97}$ & 1 & 1 & 0 & 2 & 1 & 1 & 1 & $7(8)$ \\
Gutiérrez-Hermosillo $2013^{88}$ & 1 & 1 & $\ldots$ & 0 & 1 & $\ldots$ & $\ldots$ & $3(5)$ \\
Zhen $2013^{80}$ & 0 & 1 & $\ldots$ & 2 & 1 & $\ldots$ & $\ldots$ & $4(5)$ \\
Pearce $2012^{23}$ & 1 & 1 & $\ldots$ & 2 & 1 & $\ldots$ & $\ldots$ & $5(5)$ \\
Zhong $2012^{26}$ & 1 & 1 & $\ldots$ & 1 & 1 & $\ldots$ & $\ldots$ & $4(5)$ \\
Hayashi $2011^{30}$ & 0 & 0 & $\ldots$ & 0 & 1 & $\ldots$ & $\ldots$ & $1(5)$ \\
Imamine $2011^{34}$ & 1 & 1 & 1 & 2 & 1 & 1 & 1 & $8(8)$ \\
\hline
\end{tabular}


Table S5.4 (continued)

\begin{tabular}{|c|c|c|c|c|c|c|c|c|}
\hline Reference & S1 & S2 & S3 $^{*}$ & C1 & 01 & O2* & $03^{*}$ & $\begin{array}{l}\text { Total score } \\
\text { (max) }\end{array}$ \\
\hline Reijmer $2011^{38}$ & 1 & 1 & 1 & 1 & 1 & 1 & 1 & $7(8)$ \\
\hline Kadoi $2011^{39}$ & 0 & 1 & 1 & 0 & 1 & 0 & 1 & $4(8)$ \\
\hline Rizzo $2010^{40}$ & 0 & 1 & $\ldots$ & 2 & 1 & $\ldots$ & $\ldots$ & $4(5)$ \\
\hline Abbatecola 201042 & 0 & 1 & $\ldots$ & 1 & 1 & $\ldots$ & $\ldots$ & $3(5)$ \\
\hline Shimada $2010^{44}$ & 0 & 1 & $\ldots$ & 0 & 1 & $\ldots$ & $\ldots$ & $2(5)$ \\
\hline Cukierman-Yaffe 200948 & 0 & 1 & $\ldots$ & 2 & 1 & $\ldots$ & $\ldots$ & $4(5)$ \\
\hline Tiehuis $2009^{51}$ & 0 & 1 & $\ldots$ & 2 & 1 & $\ldots$ & $\ldots$ & $4(5)$ \\
\hline Kim $2008^{54}$ & 1 & 1 & $\ldots$ & 0 & 1 & $\ldots$ & $\ldots$ & $3(5)$ \\
\hline Obidi $2008^{55}$ & 0 & 0 & $\ldots$ & 0 & 1 & $\ldots$ & $\ldots$ & $1(5)$ \\
\hline Manschot $2007^{58}$ & 1 & 1 & $\ldots$ & 2 & 1 & $\ldots$ & $\ldots$ & $5(5)$ \\
\hline Akisaki $2006^{63}$ & 1 & 1 & $\ldots$ & 0 & 1 & $\ldots$ & $\ldots$ & $3(5)$ \\
\hline Shorr $2006^{64}$ & 1 & 1 & $\ldots$ & 2 & 1 & $\ldots$ & $\ldots$ & $5(5)$ \\
\hline Gallacher $2005^{66}$ & 0 & 1 & $\ldots$ & 1 & 1 & $\ldots$ & $\ldots$ & $3(5)$ \\
\hline Vanhanen $1999^{72}$ & 0 & 1 & $\ldots$ & 0 & 1 & $\ldots$ & $\ldots$ & $2(5)$ \\
\hline Zaslavsky $1995^{75}$ & 0 & 1 & $\ldots$ & 0 & 1 & $\ldots$ & $\ldots$ & $2(5)$ \\
\hline Kalmijn $1995^{76}$ & 0 & 1 & $\ldots$ & 2 & 1 & $\ldots$ & $\ldots$ & $4(5)$ \\
\hline \multicolumn{9}{|c|}{ Fasting insulin levels $\&$ cognitive function } \\
\hline Umegaki $2014^{93}$ & 1 & 1 & 1 & 1 & 1 & 1 & 1 & $7(8)$ \\
\hline Reijmer $2011^{38}$ & 1 & 1 & 1 & 1 & 1 & 1 & 1 & $7(8)$ \\
\hline Abbatecola $2010^{42}$ & 0 & 1 & $\ldots$ & 1 & 1 & $\ldots$ & $\ldots$ & $3(5)$ \\
\hline Abbatecola $2010^{42}$ & 0 & 1 & 1 & 2 & 1 & 0 & 1 & $6(8)$ \\
\hline Tiehuis $2009^{51}$ & 0 & 1 & $\ldots$ & 2 & 1 & $\ldots$ & $\ldots$ & $4(5)$ \\
\hline Umegaki $2008^{52}$ & 1 & 1 & $\ldots$ & 2 & 1 & $\ldots$ & $\ldots$ & $5(5)$ \\
\hline Manschot $2007^{58}$ & 1 & 1 & $\ldots$ & 2 & 1 & $\ldots$ & $\ldots$ & $5(5)$ \\
\hline Akisaki $2006^{63}$ & 1 & 1 & $\ldots$ & 0 & 1 & $\ldots$ & $\ldots$ & $3(5)$ \\
\hline Luchsinger $2004^{91}$ & 1 & 1 & 1 & 2 & 1 & 1 & 1 & $8(8)$ \\
\hline \multicolumn{9}{|c|}{ HOMA-IR \& cognitive function } \\
\hline Pearce $2012^{23}$ & 1 & 1 & $\ldots$ & 2 & 1 & $\ldots$ & $\ldots$ & $5(5)$ \\
\hline Pearce $2012^{23}$ & 1 & 1 & 1 & 2 & 1 & 0 & 0 & $6(8)$ \\
\hline Benedict $2012^{25}$ & 0 & 1 & $\ldots$ & 2 & 1 & $\ldots$ & $\ldots$ & $4(5)$ \\
\hline Tiehuis $2009^{51}$ & 0 & 1 & $\ldots$ & 2 & 1 & $\ldots$ & $\ldots$ & $4(5)$ \\
\hline Manschot $2007^{58}$ & 1 & 1 & $\cdots$ & 2 & 1 & $\cdots$ & $\cdots$ & $5(5)$ \\
\hline \multicolumn{9}{|c|}{ Other glucose-related variables $\&$ cognition } \\
\hline Cui $2014^{96}$ & 1 & 1 & $\ldots$ & 2 & 1 & $\ldots$ & $\ldots$ & $5(5)$ \\
\hline Exalto $2013^{89}$ & 1 & 1 & 1 & 2 & 1 & 1 & 1 & $8(8)$ \\
\hline Crane $2013^{86}$ & 1 & 1 & 1 & 2 & 1 & 1 & 1 & $8(8)$ \\
\hline Zhong $2012^{26}$ & 1 & 1 & $\ldots$ & 1 & 1 & $\ldots$ & $\ldots$ & $4(5)$ \\
\hline Kadoi $2011^{39}$ & 0 & 1 & 1 & 0 & 1 & 0 & 1 & $4(8)$ \\
\hline Rizzo $2010^{40}$ & 0 & 1 & $\ldots$ & 2 & 1 & $\ldots$ & $\ldots$ & $4(5)$ \\
\hline Perlmuter 200946 & 0 & 1 & $\ldots$ & 1 & 1 & $\ldots$ & $\ldots$ & $3(5)$ \\
\hline Dore $2009^{49}$ & 1 & 1 & $\ldots$ & 0 & 1 & $\ldots$ & $\cdots$ & $3(5)$ \\
\hline Abbatecola $2006^{60}$ & 0 & 1 & $\ldots$ & 2 & 1 & $\ldots$ & $\ldots$ & $4(5)$ \\
\hline Ryan $2006^{61}$ & 0 & 1 & 1 & 0 & 1 & 0 & 1 & $4(8)$ \\
\hline Vanhanen $1999^{72}$ & 0 & 1 & $\ldots$ & 0 & 1 & $\ldots$ & $\ldots$ & $2(5)$ \\
\hline Naor $1997^{73}$ & 0 & 1 & $\ldots$ & 0 & 1 & $\ldots$ & $\ldots$ & $2(5)$ \\
\hline Malacco $1992^{78}$ & 0 & 1 & $\ldots$ & 0 & 1 & $\ldots$ & $\ldots$ & $2(5)$ \\
\hline
\end{tabular}


Table S5.4 (continued)

\begin{tabular}{|c|c|c|c|c|c|c|c|c|}
\hline Reference & S1 & $\mathbf{S 2}$ & S3 $^{*}$ & C1 & 01 & O2* & $\mathrm{O3}^{*}$ & $\begin{array}{l}\text { Total score } \\
\text { (max) }\end{array}$ \\
\hline \multicolumn{9}{|c|}{ Glucose-lowering treatment $\&$ cognition } \\
\hline Huang 201495 & 0 & 1 & 1 & 1 & 1 & 1 & 1 & $6(8)$ \\
\hline Ng $2014^{94}$ & 1 & 1 & $\ldots$ & 2 & 1 & $\ldots$ & $\ldots$ & $5(5)$ \\
\hline Ng $2014{ }^{94}$ & 1 & 1 & 1 & 2 & 1 & 1 & 0 & $7(8)$ \\
\hline Exalto $2013^{89}$ & 1 & 1 & 1 & 2 & 1 & 1 & 1 & $8(8)$ \\
\hline Fei $2013^{85}$ & 1 & 0 & $\ldots$ & 1 & 1 & $\ldots$ & $\ldots$ & $3(5)$ \\
\hline Mayeda $2013^{83}$ & 1 & 1 & 1 & 2 & 1 & 1 & 1 & $8(8)$ \\
\hline Seaquist $2013^{82}$ & 0 & 1 & $\ldots$ & 2 & 1 & $\ldots$ & $\ldots$ & $4(5)$ \\
\hline Seaquist $2013^{82}$ & 0 & 1 & 1 & 2 & 1 & 0 & 1 & $6(8)$ \\
\hline Yaffe $2013^{81}$ & 1 & 1 & 1 & 2 & 1 & 1 & 1 & $8(8)$ \\
\hline Umegaki 201227 & 1 & 1 & 1 & 1 & 1 & 1 & 1 & $7(8)$ \\
\hline Espeland $2011^{31}$ & 0 & 1 & 1 & 2 & 1 & 1 & 0 & $6(8)$ \\
\hline Hsu $2011^{32}$ & 1 & 1 & 1 & 1 & 1 & 1 & 1 & $7(8)$ \\
\hline Parikh $2011^{37}$ & 0 & 1 & 1 & 1 & 1 & 0 & 1 & $5(8)$ \\
\hline Alencar $2010^{43}$ & 1 & 0 & $\ldots$ & 0 & 1 & $\ldots$ & $\ldots$ & $2(5)$ \\
\hline Plastino $2010^{45}$ & 0 & 1 & $\ldots$ & 1 & 1 & $\ldots$ & $\ldots$ & $3(5)$ \\
\hline Plastino $2010^{45}$ & 0 & 1 & 1 & 1 & 1 & 0 & 0 & $4(8)$ \\
\hline Saczynski $2008^{53}$ & 0 & 1 & $\ldots$ & 2 & 1 & $\ldots$ & $\ldots$ & $4(5)$ \\
\hline Irie $2008^{57}$ & 1 & 1 & 1 & 0 & 1 & 1 & 1 & $6(8)$ \\
\hline Logroscino $2004^{68}$ & 0 & 1 & $\ldots$ & 0 & 1 & $\ldots$ & $\ldots$ & $2(5)$ \\
\hline Wu $2003^{69}$ & 1 & 1 & 1 & 1 & 1 & 0 & 1 & $6(8)$ \\
\hline Crooks 200370 & 0 & 1 & $\ldots$ & 0 & 1 & $\ldots$ & $\ldots$ & $2(5)$ \\
\hline \multicolumn{9}{|c|}{ Hypoglycaemic events \& cognition } \\
\hline Feinkohl $2013^{84}$ & 1 & 1 & 1 & 2 & 1 & 1 & 1 & $8(8)$ \\
\hline Yaffe $2013^{81}$ & 1 & 1 & 1 & 2 & 1 & 1 & 1 & $8(8)$ \\
\hline $\operatorname{Lin} 2012^{22}$ & 1 & 1 & 1 & 1 & 1 & 1 & 1 & $7(8)$ \\
\hline Umegaki $2012^{27}$ & 1 & 1 & 1 & 1 & 1 & 1 & 1 & $7(8)$ \\
\hline Aung $2011^{28}$ & 1 & 1 & $\ldots$ & 2 & 1 & $\ldots$ & $\ldots$ & $5(5)$ \\
\hline Whitmer $2009^{47}$ & 1 & 1 & 1 & 2 & 1 & 1 & 1 & $8(8)$ \\
\hline Bruce $2009^{50}$ & 1 & 1 & $\ldots$ & 2 & 1 & $\ldots$ & $\ldots$ & $5(5)$ \\
\hline Bruce $2009^{50}$ & 1 & 1 & 1 & 2 & 1 & 0 & 1 & $7(8)$ \\
\hline Mogi $2004^{67}$ & 1 & 1 & $\cdots$ & 0 & 1 & $\cdots$ & $\ldots$ & $3(5)$ \\
\hline \multicolumn{9}{|c|}{ HbA1c \& brain MRI abnormalities } \\
\hline Bryan $20144^{107}$ & 1 & 1 & $\ldots$ & 2 & 1 & $\ldots$ & $\ldots$ & $5(5)$ \\
\hline Hsu $2012^{100}$ & 0 & 1 & $\ldots$ & 0 & 1 & $\ldots$ & $\ldots$ & $2(5)$ \\
\hline Hayashi $2011^{30}$ & 0 & 0 & $\ldots$ & 0 & 1 & $\ldots$ & $\ldots$ & $1(5)$ \\
\hline Imamine $2011^{34}$ & 1 & 1 & 1 & 2 & 1 & 1 & 1 & $8(8)$ \\
\hline Bresser de $2010^{101}$ & 1 & 1 & 1 & 2 & 1 & 1 & 1 & $8(8)$ \\
\hline Anan $2010^{102}$ & 0 & 1 & $\ldots$ & 0 & 1 & $\ldots$ & $\ldots$ & $2(5)$ \\
\hline Brundel $2010^{103}$ & 0 & 1 & $\ldots$ & 2 & 1 & $\ldots$ & $\ldots$ & $4(5)$ \\
\hline Kumar $2008^{105}$ & 0 & 1 & $\ldots$ & 0 & 0 & $\ldots$ & $\ldots$ & $1(5)$ \\
\hline Manschot $2006^{62}$ & 1 & 1 & $\ldots$ & 2 & 1 & $\ldots$ & $\ldots$ & $5(5)$ \\
\hline Eguchi $2006^{106}$ & 1 & 0 & $\ldots$ & 2 & 0 & $\ldots$ & $\ldots$ & $3(5)$ \\
\hline Akisaki $2006^{63}$ & 1 & 1 & $\ldots$ & 0 & 1 & $\ldots$ & $\ldots$ & $3(5)$ \\
\hline \multicolumn{9}{|c|}{ Fasting glucose levels \& brain MRI abnormalities } \\
\hline Bryan 2014107 & 1 & 1 & $\ldots$ & 2 & 1 & $\ldots$ & $\ldots$ & $5(5)$ \\
\hline Hsu $2012^{100}$ & 0 & 1 & $\ldots$ & 0 & 1 & $\ldots$ & $\ldots$ & $2(5)$ \\
\hline
\end{tabular}


Table S5.4 (continued)

\begin{tabular}{|c|c|c|c|c|c|c|c|c|}
\hline Reference & S1 & S2 & S3 $^{*}$ & C1 & 01 & $\mathrm{O2}^{*}$ & O3 $^{*}$ & $\begin{array}{l}\text { Total score } \\
\text { (max) }\end{array}$ \\
\hline Hayashi $2011^{30}$ & 0 & 0 & $\ldots$ & 0 & 1 & $\ldots$ & $\ldots$ & $1(5)$ \\
\hline Imamine $2011^{34}$ & 1 & 1 & 1 & 2 & 1 & 1 & 1 & $8(8)$ \\
\hline Anan $2010^{102}$ & 0 & 1 & $\ldots$ & 0 & 1 & $\ldots$ & $\ldots$ & $2(5)$ \\
\hline Tiehuis $2008^{104}$ & 0 & 1 & $\ldots$ & 2 & 1 & $\ldots$ & $\ldots$ & $4(5)$ \\
\hline Manschot $2007^{58}$ & 1 & 1 & $\ldots$ & 2 & 1 & $\ldots$ & $\ldots$ & $5(5)$ \\
\hline Akisaki $2006^{63}$ & 1 & 1 & $\ldots$ & 0 & 1 & $\ldots$ & $\ldots$ & $3(5)$ \\
\hline \multicolumn{9}{|c|}{ Fasting insulin levels \& brain MRI abnormalities } \\
\hline Imamine $2011^{34}$ & 1 & 1 & 1 & 2 & 1 & 1 & 1 & $8(8)$ \\
\hline Anan $2010^{102}$ & 0 & 1 & $\ldots$ & 0 & 1 & $\ldots$ & $\ldots$ & $2(5)$ \\
\hline Manschot $2007^{58}$ & 1 & 1 & $\ldots$ & 2 & 1 & $\ldots$ & $\ldots$ & $5(5)$ \\
\hline Akisaki $2006^{63}$ & 1 & 1 & $\ldots$ & 0 & 1 & $\ldots$ & $\ldots$ & $3(5)$ \\
\hline \multicolumn{9}{|c|}{ HOMA-IR \& brain MRI abnormalities } \\
\hline Benedict $2012^{25}$ & 0 & 1 & $\ldots$ & 2 & 1 & $\ldots$ & $\ldots$ & $4(5)$ \\
\hline Anan $2010^{102}$ & 0 & 1 & $\ldots$ & 0 & 1 & $\ldots$ & $\ldots$ & $2(5)$ \\
\hline Tiehuis $2008^{104}$ & 0 & 1 & $\ldots$ & 2 & 1 & $\ldots$ & $\ldots$ & $4(5)$ \\
\hline Manschot $2007^{58}$ & 1 & 1 & $\cdots$ & 2 & 1 & $\ldots$ & $\cdots$ & $5(5)$ \\
\hline \multicolumn{9}{|c|}{ Other glucose-related variables \& brain MRI abnormalities } \\
\hline Cui $2014^{96}$ & 1 & 1 & $\ldots$ & 2 & 1 & $\ldots$ & $\ldots$ & $5(5)$ \\
\hline \multicolumn{9}{|c|}{ Glucose-lowering treatment \& brain MRI abnormalities } \\
\hline Anan $2010^{102}$ & 0 & 1 & $\ldots$ & 0 & 1 & $\ldots$ & $\ldots$ & $2(5)$ \\
\hline Tiehuis $2008^{104}$ & 0 & 1 & $\ldots$ & 2 & 1 & $\ldots$ & $\ldots$ & $4(5)$ \\
\hline Eguchi $2006^{106}$ & 1 & 0 & $\ldots$ & 2 & 0 & $\ldots$ & $\ldots$ & $3(5)$ \\
\hline
\end{tabular}

Studies are listed according to year of publication. ${ }^{*}$ Items $\mathrm{S} 3, \mathrm{O} 2$, and $\mathrm{O} 3$ are only applicable in case of longitudinal analyses. Abbreviations: $\mathrm{S}=$ selection; $\mathrm{C}=$ comparability; $\mathrm{O}=$ outcome; $\mathrm{HOMA}-\mathrm{IR}=$ Homeostasis Model Assessment of insulin resistance; MRI = Magnetic Resonance Imaging. 
Table S5.5 Risk of bias for randomised trials

\begin{tabular}{|c|c|c|}
\hline Bias & $\begin{array}{l}\text { Author's } \\
\text { judgment (SG) }\end{array}$ & Support for judgment \\
\hline \multicolumn{3}{|l|}{ Cukierman-Yaffe $2014^{98}$} \\
\hline $\begin{array}{l}\text { Random sequence generation } \\
\text { (selection bias) }\end{array}$ & Low risk & $\begin{array}{l}\text { Quote: "were randomly assigned by an } \\
\text { automated telephone randomization system } \\
\text { (with randomly varying block sizes)" }\end{array}$ \\
\hline $\begin{array}{l}\text { Allocation concealment } \\
\text { (selection bias) }\end{array}$ & Low risk & $\begin{array}{l}\text { Quote: "were randomly assigned by an } \\
\text { automated telephone randomization system } \\
\text { (with randomly varying block sizes)" }\end{array}$ \\
\hline $\begin{array}{l}\text { Blinding of participants and researchers } \\
\text { (performance bias) }\end{array}$ & High risk & Open-label \\
\hline $\begin{array}{l}\text { Blinding of outcome assessment } \\
\text { (detection bias) }\end{array}$ & Low risk & $\begin{array}{l}\text { Quote: "outcome adjudicators and data } \\
\text { analysts were masked to treatment } \\
\text { allocation" }\end{array}$ \\
\hline $\begin{array}{l}\text { Incomplete outcome data } \\
\text { (attrition bias) }\end{array}$ & Low risk & $\begin{array}{l}\text { Quote: "all analyses were restricted to those } \\
\text { participants who had a cognitive } \\
\text { measurement at both baseline and at least } \\
\text { one follow-up" }\end{array}$ \\
\hline $\begin{array}{l}\text { Selective reporting } \\
\text { (reporting bias) }\end{array}$ & Low risk & All outcomes were reported \\
\hline Other bias & Unclear risk & $\begin{array}{l}\text { Insufficient information to assess whether } \\
\text { or not other forms of bias might be present }\end{array}$ \\
\hline
\end{tabular}

\section{Koekkoek $2012^{29}$}

Random sequence generation

(selection bias)

Allocation concealment

(selection bias)

Blinding of participants and researchers (performance bias)

Blinding of outcome assessment (detection bias)

Incomplete outcome data (attrition bias)
Low risk

Low risk

Unclear risk

Unclear risk

High risk
Quote: "general practices were randomly allocated to intensive multifactorial treatment or routine care using computergenerated random numbers".

Central allocation via computer-generated numbers.

Quote: "allocation was concealed from participants".

Comment: participants were blinded, unclear whether or not researchers are blinded.

Quote: "the tests were administered in a fixed order at the participant's home by neuropsychologists and neuropsychologists in training".

Comment: unclear whether or not neuropsychologists (in training) were aware of allocation.

Loss to follow-up was reported and specified.

Participants versus non-participants at the $2^{\text {nd }}$ neuropsychological assessment:

Quote: “cognitive performance at the $1^{\text {st }}$ neuropsychological assessment differed with a mean difference on the composite $z-$ score of $-0.18^{\prime \prime}$. 
Table S5.5 (continued)

\begin{tabular}{lll}
\hline Bias & $\begin{array}{l}\text { Author's } \\
\text { judgment (SG) }\end{array}$ & Support for judgment \\
&
\end{tabular}

Selective reporting

(reporting bias)

Other bias

Launer $2011^{33}$

Random sequence generation

(selection bias)

Allocation concealment

(selection bias)

Blinding of participants and researchers

(performance bias)

Blinding of outcome assessment

(detection bias)

Incomplete outcome data

(attrition bias)
Low risk

Unclear risk

Low risk

Low risk

High risk

High risk

Low risk

Selective reporting

(reporting bias)
Participants versus non-participants at the

$2^{\text {nd }}$ neuropsychological assessment:

Quote: "In the intensive treatment group

participants of the second

neuropsychological assessment scored

higher than non-participants on the

composite score of the first

neuropsychological assessment".

All outcomes were reported

Quote: "Randomisation was stratified according to practice organization". Comment: patient populations might differ among practice organizations, thereby introducing selection bias

Quote: "A computer at the central coordinating centre generated unique randomisation sequences for every clinical site and electronically verified exclusion and inclusion criteria for every individual before assigning a treatment group" Quotes: "A computer at the central coordinating centre" and "Clinical staff implemented the randomisation via secure access to the ACCORD trial website". Open-label

Open-label

Loss to follow up was reported, specified and did not markedly differ between the two treatment arms. The authors argued that is unlikely that selective loss to followup influenced the results.

Quote: "For the deaths to have affected our conclusion in favour of intensive treatment, substantially higher follow-up cognitive scores would have been needed from the 47 people who died in the intensive group than from the 39 in the standard group. We think this would be unlikely, because it assumes that those on intensive therapy who died would have experienced a greater treatment-group effect than those who survived."

All outcomes were reported. 


\section{Chapter 5}

Table S5.5 (continued)

\begin{tabular}{lll}
\hline Bias & $\begin{array}{l}\text { Author's } \\
\text { judgment (SG) }\end{array}$ & Support for judgment
\end{tabular}

Other bias

Luchsinger $2011^{36}$

Random sequence generation

(selection bias)

Allocation concealment

(selection bias)

Blinding of participants and researchers

(performance bias)

Blinding of outcome assessment

(detection bias)

Incomplete outcome data

(attrition bias)

Selective reporting

(reporting bias)

Other bias
High risk

Unclear

Unclear

Low risk

Low risk

Low risk

Unclear

Low risk
Quote: "The intensive intervention was stopped on Feb 6, 2008, when an increased risk (hazard ratio $1.22,95 \% \mathrm{Cl} 1.01$ - 1.46) for mortality was reported; participants in that group were moved to the standard glycaemic treatment".

Quote: "participants were randomized by PCP (primary care provider) - clusters".

Quote from the design and rationale article of the IDEATel [ref (Shea $S$ et al, 2002)]:

"Each subject enrolled in the study has a primary care physician, and randomization is within blocks defined by PCP patient panels".

Not specified

Not specified, both it is unlikely that participants and primary care providers were blinded to allocation.

Blinded

Loss to follow-up was reported but not specified.

Quote: "Analyses used an intent-to-treat approach".

All outcome were reported.

There might be performance bias because no information is provided on the uptake of the guidelines by primary care providers.

Quote: "PCPs were mailed diabetes care guidelines".

Not specified in paper, but stated in design and rationale article: "computer-based randomisation".

Not specified in paper, but stated in design and rationale article: "central computerbased". 
Table S5.5 (continued)

\begin{tabular}{|c|c|c|}
\hline Bias & $\begin{array}{l}\text { Author's } \\
\text { judgment (SG) }\end{array}$ & Support for judgment \\
\hline $\begin{array}{l}\text { Blinding of participants and researchers } \\
\text { (performance bias) }\end{array}$ & Unclear & Not specified, but likely to be open label. \\
\hline $\begin{array}{l}\text { Blinding of outcome assessment } \\
\text { (detection bias) }\end{array}$ & Low risk & $\begin{array}{l}\text { Not specified in paper, but stated in design } \\
\text { and rationale article: "an Endpoint } \\
\text { Adjudication Committee blind to treatment } \\
\text { assignment, will review all suspected } \\
\text { primary outcomes". }\end{array}$ \\
\hline $\begin{array}{l}\text { Incomplete outcome data } \\
\text { (attrition bias) }\end{array}$ & Low risk & $\begin{array}{l}\text { Loss to follow-up is mentioned and } \\
\text { specified. }\end{array}$ \\
\hline $\begin{array}{l}\text { Selective reporting } \\
\text { (reporting bias) }\end{array}$ & Unclear & $\begin{array}{l}\text { Cognition is shortly reported as an outcome } \\
\text { measure, while it is not described as an } \\
\text { outcome measure of interest in the } \\
\text { Methods section. }\end{array}$ \\
\hline Other bias & Unclear & $\begin{array}{l}\text { Insufficient information to assess whether } \\
\text { or not other forms of bias might be present. }\end{array}$ \\
\hline
\end{tabular}

\section{Abbatecola $2006^{59}$}

Random sequence generation (selection bias)

Quote: "patients were randomly selected to be treated with repaglinide $(n=77)$ or glibenclamide $(\mathrm{n}=79)^{\prime \prime}$.

Allocation concealment

Unclear risk

Not further specified.

(selection bias)

Blinding of participants and researchers

High risk

Not specified.

(performance bias)

Blinding of outcome assessment

(detection bias)

Low risk

Open study

Blinded

Incomplete outcome data

Low risk (attrition bias)

Quote: "All cognitive evaluation were made by physicians who were unaware of both study design and patient treatment allocation".

Loss to follow-up is mentioned and specified, as well as participants who violated the study protocol. The loss to follow-up was small and did not markedly differ between the treatment group. In addition, an intention-to-treat analyses was performed.

Selective reporting

Low risk All outcomes were reported.

(reporting bias)

Other bias

Unclear 
Table S5.5 (continued)

\begin{tabular}{|c|c|c|}
\hline Bias & $\begin{array}{l}\text { Author's } \\
\text { judgment (SG) }\end{array}$ & Support for judgment \\
\hline \multicolumn{3}{|l|}{ Ryan $2006^{61}$} \\
\hline $\begin{array}{l}\text { Random sequence generation } \\
\text { (selection bias) }\end{array}$ & Low risk & $\begin{array}{l}\text { Randomisation was based on a computer- } \\
\text { generated allocation sequence. }\end{array}$ \\
\hline $\begin{array}{l}\text { Allocation concealment } \\
\text { (selection bias) }\end{array}$ & Unclear risk & Not further specified \\
\hline $\begin{array}{l}\text { Blinding of participants and researchers } \\
\text { (performance bias) }\end{array}$ & Low risk & Double blinded \\
\hline $\begin{array}{l}\text { Blinding of outcome assessment } \\
\text { (detection bias) }\end{array}$ & Low risk & Double blinded \\
\hline $\begin{array}{l}\text { Incomplete outcome data } \\
\text { (attrition bias) }\end{array}$ & Low risk & $\begin{array}{l}\text { Loss to follow-up was reported, specified, } \\
\text { and did not markedly differ between the } \\
\text { treatment groups. An intention-to-treat } \\
\text { analysis was conducted. }\end{array}$ \\
\hline $\begin{array}{l}\text { Selective reporting } \\
\text { (reporting bias) }\end{array}$ & Low risk & All outcome measures were reported. \\
\hline Other bias & Unclear & $\begin{array}{l}\text { Insufficient information to assess whether } \\
\text { or not other forms of bias might be present. }\end{array}$ \\
\hline
\end{tabular}




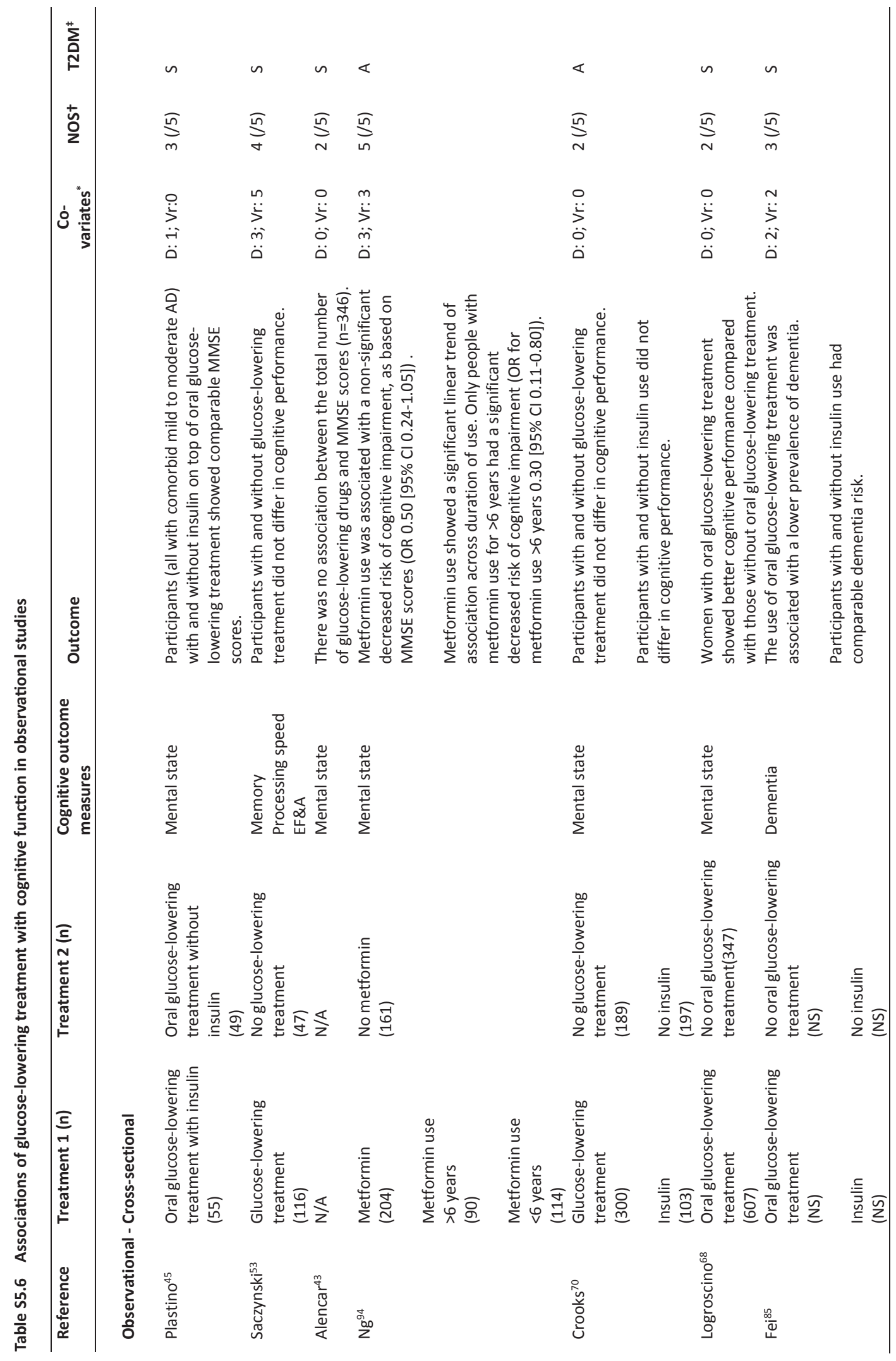




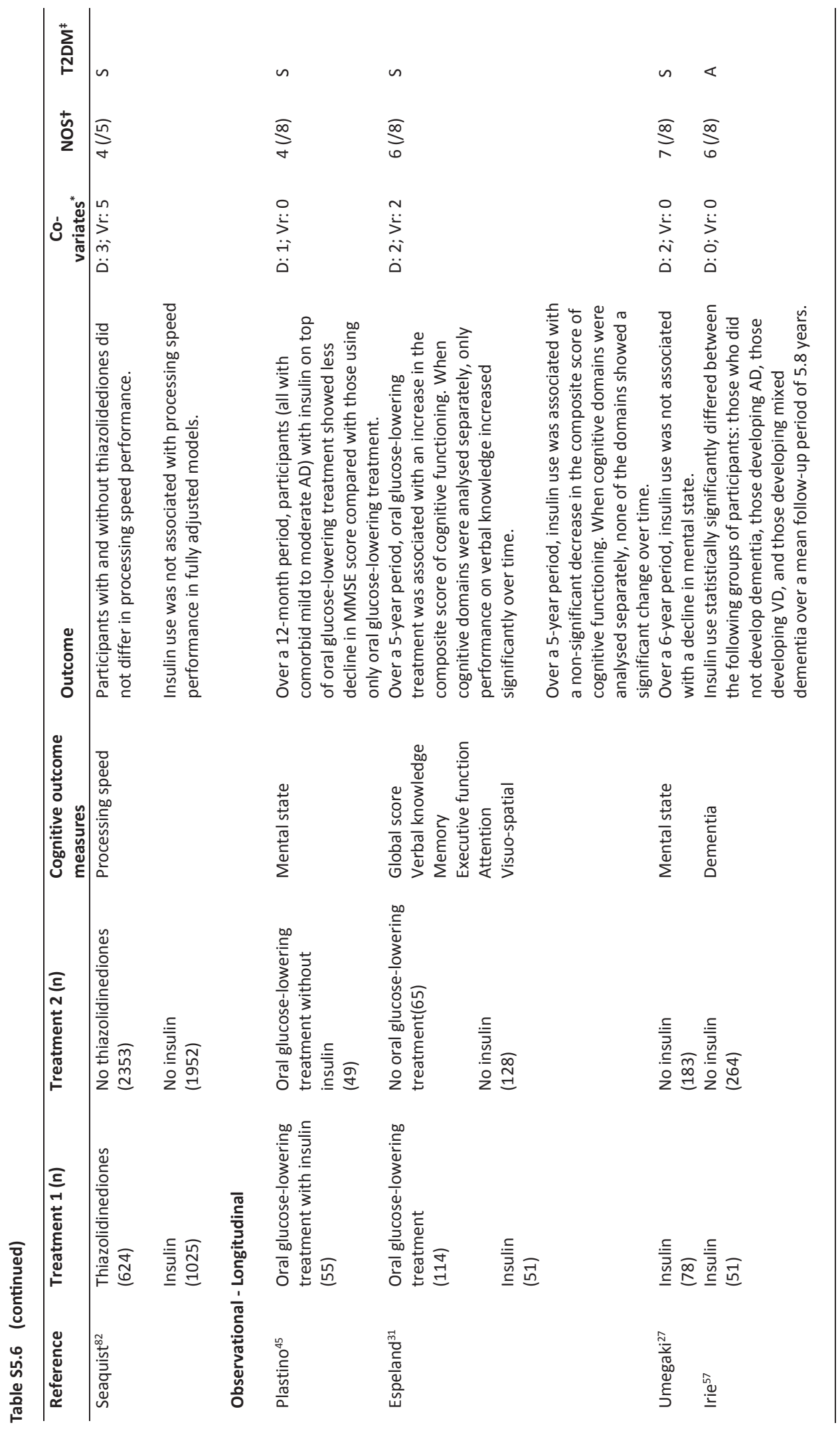




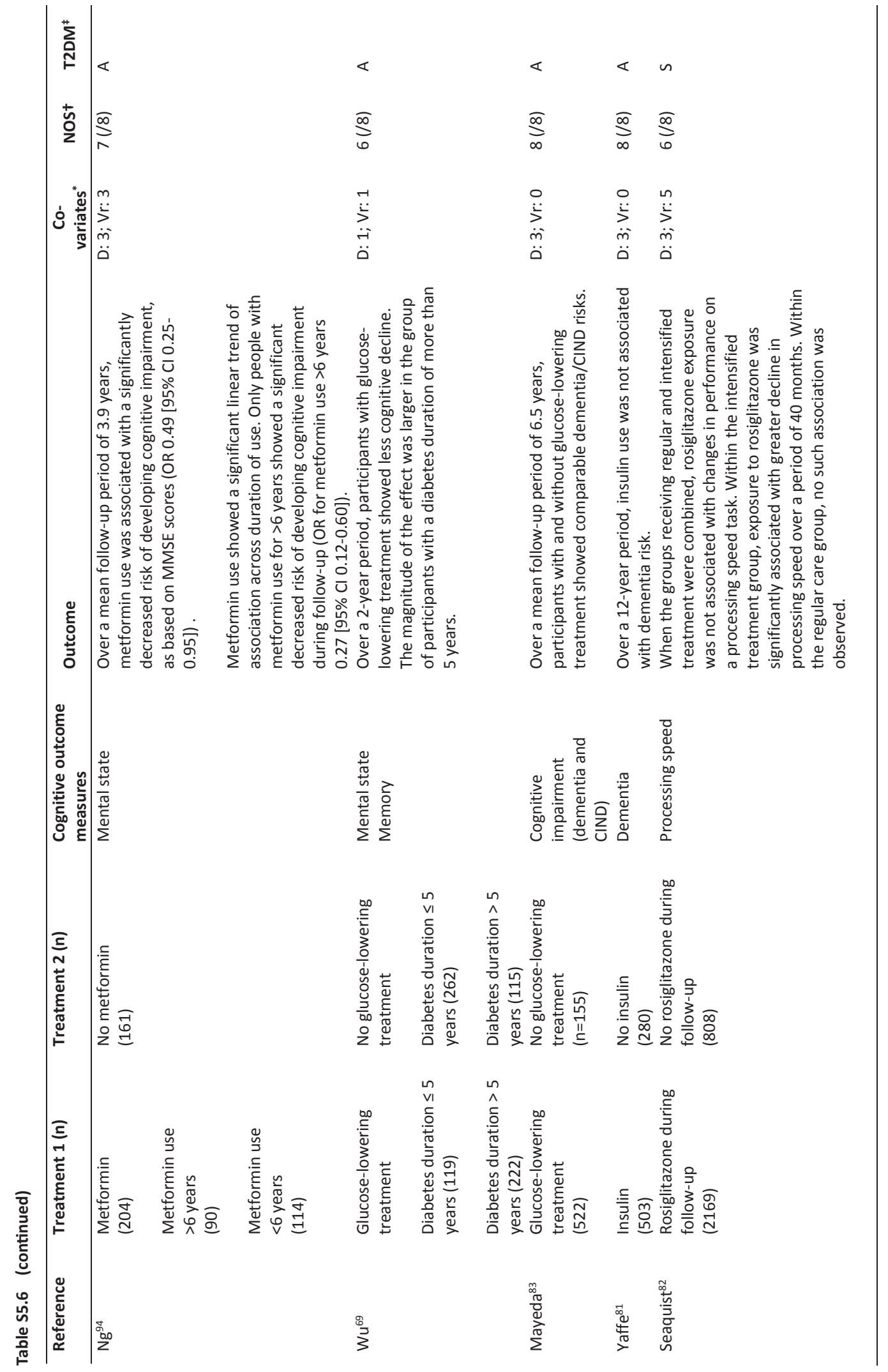




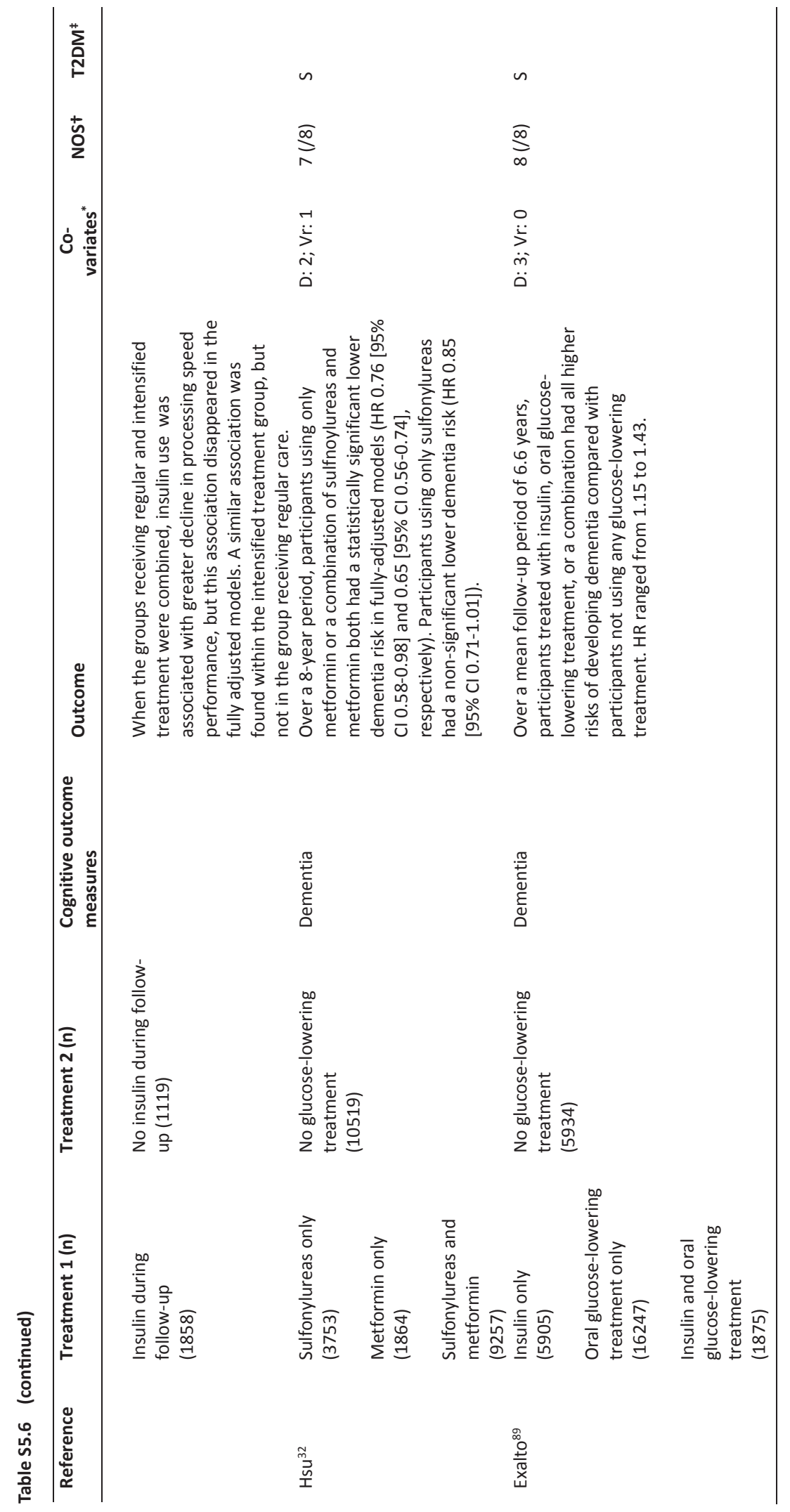




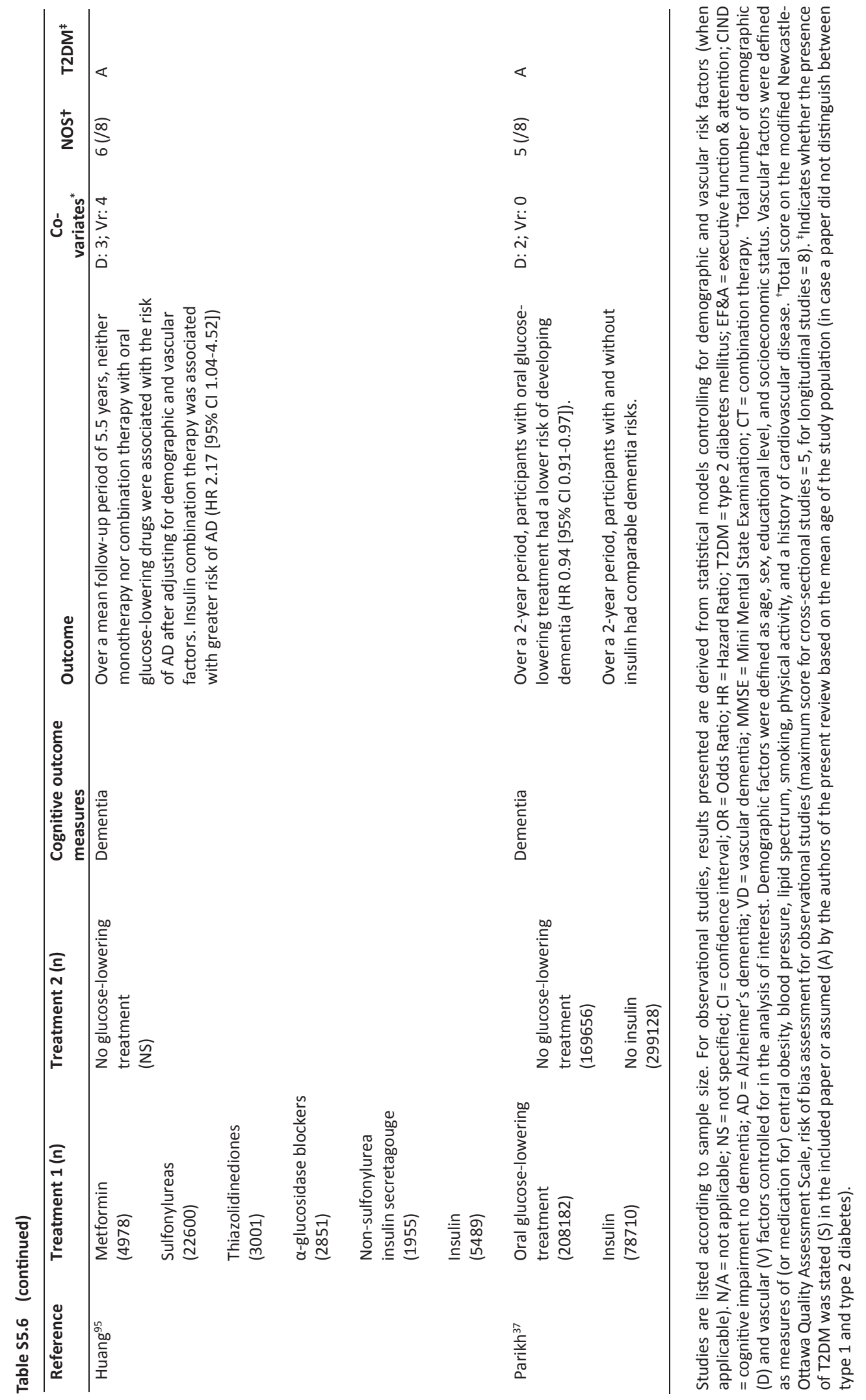


Figure S5.1 Summary of results of included studies with exclusion of studies at high risk of bias

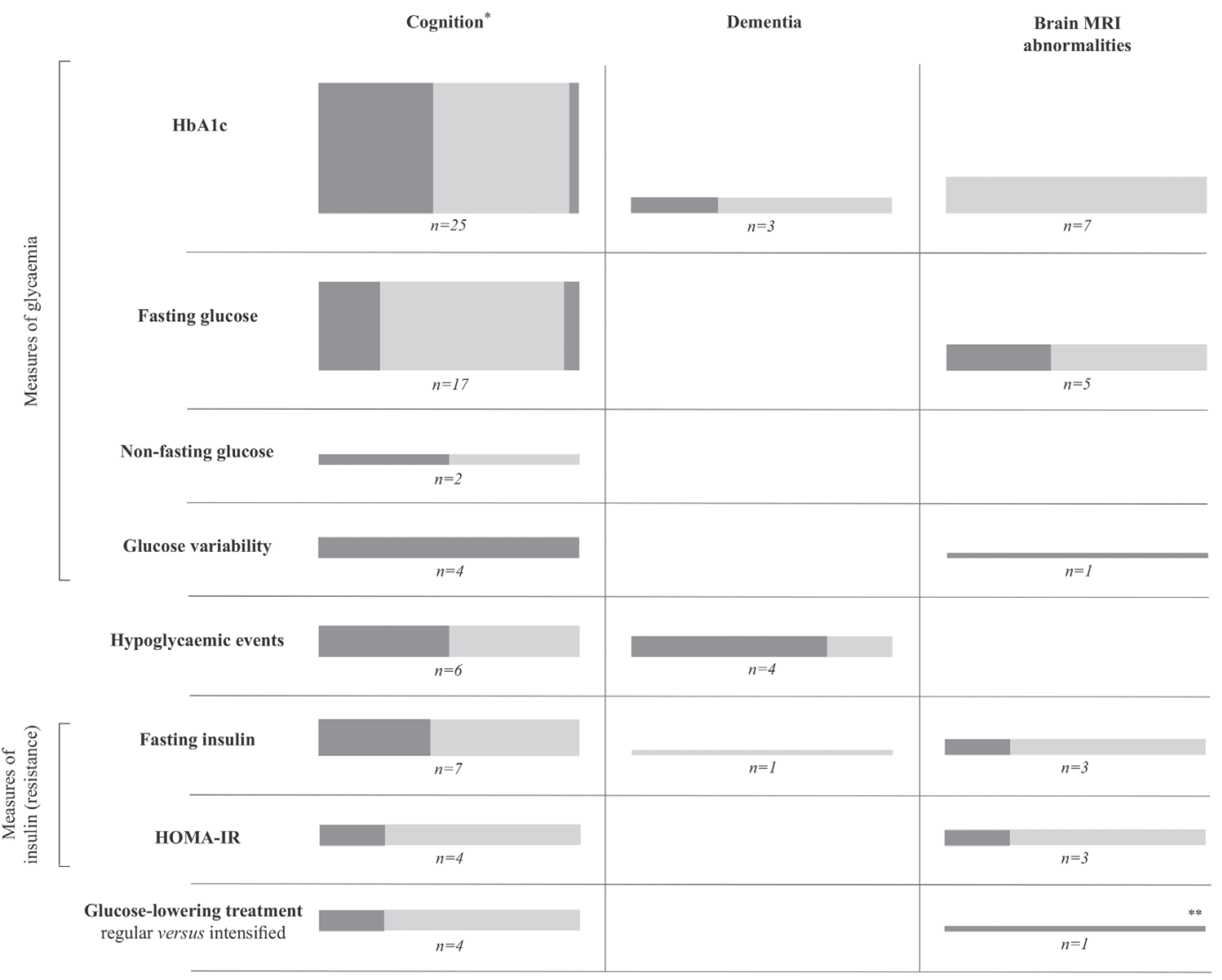

worse glycaemic control $^{* * *}$ is associated with worse cognitive functioning, higher dementia risk, or more brain MRI abnormalities

worse glycaemic control $^{* * *}$ is not associated with cognitive functioning, dementia risk, or brain MRI abnormalities

worse glycaemic control $^{* * *}$ is associated with better cognitive functioning, lower dementia risk, or fewer brain MRI abnormalities

Results are derived from statistical models controlled for demographic factors en vascular risk factors (when applicable). The height of each bar indicates the amount of evidence available. Results of cross-sectional and longitudinal analyses are combined. * Cognition includes measures that reflect cognitive status (cognitive function at a single point in time) or change in cognitive function over time. ${ }^{* *}$ Intensified glucose-lowering treatment was associated with less decline in total brain volume, but more progression of white matter hyperintensities on MRI. ${ }^{* * *}$ Worse glycaemic controles is defined as high concentrations of HbA1c, fasting blood glucose, or non-fasting blood glucose; greater glucose variability; hypoglycaemic events; high fasting insulin concentration; high HOMA-IR; or achieved with glucose-lowering treatment. HOMA-IR = homeostasis model assessment of insulin resistance. 
Type 2 diabetes, cognition, and brain MRI 


\section{Chapter 6}

\section{INSULIN RESISTANCE AND COGNITIVE PERFORMANCE IN TYPE 2 DIABETES: THE MAASTRICHT STUDY}

Stefan L.C. Geijselaers, Simone J.S. Sep, Miranda T. Schram, Martin P.J. van Boxtel, Ronald M.A. Henry, Frans R.J. Verhey, Abaraham A. Kroon, Nicolaas C. Schaper, Pieter C. Dagnelie, Carla J.H. van der Kallen, Coen D.A. Stehouwer, Geert Jan Biessels 


\title{
Chapter 6
}

\begin{abstract}
Objective

Type 2 diabetes, hyperinsulinaemia, and insulin resistance are associated with cognitive impairment and dementia. Experimental studies indicate that insulin signalling in the brain is related to cognitive performance. In this cross-sectional study, we evaluated whether insulinrelated variables contribute to the variance in cognitive performance among individuals with type 2 diabetes.
\end{abstract}

\section{Research Design and Methods}

A total of 806 individuals with type 2 diabetes (mean age $62 \pm 8$ years, $67 \%$ men, $\mathrm{HbA} 1 \mathrm{c} 6.9 \pm 1.1 \%$ $\left.(52 \pm 12 \mathrm{mmol} / \mathrm{mol}), \mathrm{BMI} 29.8 \pm 5.0 \mathrm{~kg} / \mathrm{m}^{2}\right)$ completed a neuropsychological test battery assessing memory function, information processing speed, and executive function and attention. The following insulin-related variables were measured: fasting plasma insulin, C-peptide, and insulin resistance estimated using the Homeostasis Model Assessment (HOMA2-IR; only in individuals without insulin treatment; $n=641$ ). The unadjusted coefficient of determination $\left(R^{2}\right)$, obtained from multiple linear regression analyses, was used to estimate the proportion of variance in cognition explained by insulin-related variables.

\section{Results}

Sex, age, and educational level together explained $18.0 \%\left(\mathrm{R}^{2}\right)$ of the variance in memory function, $26.5 \%$ in information processing speed, and $22.8 \%$ in executive function and attention. Fasting insulin, C-peptide, or HOMA2-IR did not increase the explained variance (maximum $\Delta R^{2} 0.3 \%$, $P \geq 0.14)$. Similar results were obtained when insulin-related variables were added to models that additionally included glycaemic control, cardiovascular risk factors, and depression.

\section{Conclusions}

Our results show that measures of peripheral insulin resistance are unrelated to cognitive performance among late-middle-aged individuals with adequately controlled type 2 diabetes. 


\section{Introduction}

Type 2 diabetes is known to be associated with cognitive dysfunction. The severity of diabetes-associated cognitive changes varies, ranging from subtle cognitive decrements to mild cognitive impairment and dementia. ${ }^{1}$ Yet it is unclear why individuals with type 2 diabetes develop these cognitive changes. Given the ongoing diabetes epidemic and the increasing life-expectancy of individuals with type 2 diabetes, ${ }^{2}$ there is an urgent need to identify factors that contribute to the severity and progression of cognitive problems among individuals with type 2 diabetes.

We recently conducted a systematic review of the literature to examine whether abnormalities in glucose regulation, the hallmark of diabetes, are related to cognitive performance among individuals with type 2 diabetes. ${ }^{3}$ Based on the findings of 86 papers, we concluded that measures of glycaemia, particularly glycated hemoglobin (HbA1c) and glucose variability, are negatively, but weakly, associated with cognitive performance. We also noted that, compared to glycaemia, far less is currently known about the association of hyperinsulinaemia and insulin resistance with cognitive performance among individuals with type 2 diabetes. ${ }^{3}$ This topic is clearly worthy of further study, not only because these insulin-related measures have been found to be inversely associated with cognitive performance in individuals without diabetes (eg, ${ }^{4-}$ $\left.{ }^{6}\right)$, but especially because experimental studies have linked insulin signalling to normal brain functioning, as well as to brain disease. ${ }^{7}$

The aim of the present study was to assess whether insulin-related variables (ie, fasting insulin, C-peptide, and the Homeostasis Model Assessment of Insulin Resistance (HOMA2-IR)) contribute to the variance in cognitive performance among individuals with type 2 diabetes.

\section{Research design and methods}

\section{Study population}

We used data from The Maastricht Study, an observational prospective populationbased cohort study enriched with individuals with type 2 diabetes. The rationale and methodology have been described previously. ${ }^{8}$ In brief, the study focuses on the aetiology, pathophysiology, complications, and comorbidities of type 2 diabetes and is characterised by an extensive phenotyping approach. Eligible for participation were all individuals aged between 40 and 75 years living in the southern part of the Netherlands. Participants were recruited through mass media campaigns and from the municipal registries and the regional Diabetes Patient Registry via mailings. 
For the present study, cross-sectional data from the first 975 participants with type 2 diabetes were used, who completed the baseline survey between November 2010 and September 2013. Diabetes status was determined by a 75-g oral glucose tolerance test and defined according to the $2006 \mathrm{WHO}^{9}$ diagnostic criteria, as described in more detail before. ${ }^{8}$ Participants were also considered to have type 2 diabetes if they used glucoselowering medication without a prior diagnosis of type 1 diabetes.

The Maastricht Study was approved by the institutional medical ethical committee (NL31329.068.10) and the Minister of Health, Welfare and Sports of the Netherlands, on the basis of the Health Council's opinion (Permit 131088-105234-PG). All participants gave written informed consent.

\section{Insulin-related variables}

The following insulin-related variables were considered in the present study: fasting insulin, C-peptide, and HOMA2-IR. Both insulin and C-peptide were included as they provide similar but complementary information. Specifically, while insulin and C-peptide are secreted in equimolar amounts by the pancreatic $\beta$-cells, $C$-peptide is not extracted from the circulation by the liver, and hence its half-life is longer than that of insulin, ${ }^{10}$ which might make it a more stable and reliable reflector of insulin secretion. In addition, recent research has shown that $\mathrm{C}$-peptide interacts with insulin and may prevent neuronal loss and cognitive deficits in experimental type 1 diabetes. ${ }^{11}$ All insulin-related variables were based on venous blood samples, which were collected after an overnight fast. Serum and plasma were separated after centrifugation ( $3000 \mathrm{xg}$ for $15 \mathrm{~min}$ at $4{ }^{\circ} \mathrm{C}$ ) and were stored at $-80^{\circ} \mathrm{C}$ until the assays were performed.

Insulin and C-peptide were quantified on a Meso Scale custom duplex assay (Meso Scale Discovery, Gaithersburg, Maryland, USA). In short, 96 well-plates, with capture antibodies against insulin and C-peptide patterned on distinct spots in the same well, were supplied by the manufacturer. Samples $(10 \mu \mathrm{L} /$ well $)$, detection antibodies, and read buffer for electrochemiluminescence were applied according to manufacturer's instruction, and plates were read using a SECTOR ${ }^{\circledR} 2400$ Imager. Detection ranges of the assay were $35-25,000 \mathrm{pg} / \mathrm{mL}$ for insulin and $70-50,000 \mathrm{pg} / \mathrm{mL}$ for C-peptide. Interassay coefficients of variation for insulin and C-peptide were $10.1 \%$ and $8.2 \%$, respectively. Insulin and C-peptide were converted from $\mathrm{pg} / \mathrm{mL}$ to $\mathrm{pmol} / \mathrm{L}$ using a molar mass of $5808 \mathrm{~g}$ for insulin and 3010g for C-peptide.

Insulin resistance was estimated from fasting insulin and glucose by the Homeostasis Model Assessment of Insulin Resistance (HOMA2-IR) and calculated by using the HOMA2 calculator (version 2.2.3 for Windows; available from https://www.dtu.ox.ac. 
uk/ homacalculator). The fasting plasma glucose concentrations required to calculate HOMA2-IR were measured in venous blood samples with the enzymatic hexokinase method using two automatic analyzers (ie, the Beckman Synchron LX20 [Beckman Coulter Inc., USA] for samples obtained between November 2010 and April 2012, and the Roche Cobas 6000 [Roche Diagnostics, Mannheim, Germany] for samples obtained thereafter). Because the HOMA2-IR has not been validated for individuals using exogenous insulin, ${ }^{12}$ calculations were limited to those not treated with insulin.

\section{Cognitive performance}

A concise (30 $\mathrm{min})$ neuropsychological test battery was applied to assess cognitive performance. ${ }^{8}$ Test scores were standardised and divided into three cognitive domains (ie, memory function, executive function and attention, and information processing speed), as detailed in the Supplement. In short, memory function was evaluated using the Verbal Learning Test by calculating the mean of total immediate and delayed recall scores. The composite score for information processing speed was derived from the Stroop Colour Word Test Part I and II, the Concept Shifting Test Part A and B, and the Letter-Digit Substitution Test. Executive function and attention was assessed by the Stroop Colour Word Test Part III and the Concept Shifting Test Part C. Raw test scores were standardised using the mean and the standard deviation of the subpopulation with type 2 diabetes rather than the whole Maastricht Study population. Where appropriate, individual test scores were inverted so that higher scores indicated better cognitive performance.

\section{Covariates}

Fasting serum total and high-density lipoprotein (HDL) cholesterol were measured with standard enzymatic methods on the same auto-analyzers as used to measure glucose. $\mathrm{HbA1c}$ was measured with ion-exchange high performance liquid chromatography. Questionnaires were used to gather information on, among others, diabetes duration, smoking behaviour (never/former/current), and history of cardiovascular disease (CVD), as described in detail before. ${ }^{8}$ Personal interviews were conducted to evaluate medication $u e^{8}$ and determine educational level, which was categorised as low, intermediate, or high. ${ }^{13}$ Suspected presence of a depression was assessed with the Mini International Neuropsychiatric Interview (MINI). ${ }^{8}$ Waist circumference was measured in duplicate midway between the lower rib margin and the iliac crest at the end of expiration and recorded to the nearest $0.5 \mathrm{~cm}$. Systolic and diastolic blood pressure were calculated as the average of at least three consecutive blood pressure readings (Omron 705IT, Omron Corporation, Kyoto, Japan) performed after a minimum of 10 min seated rest. Data on 24-h ambulatory blood pressure monitoring (WatchBP03, Microlife AG, Widnau, Switzerland $)^{8}$ were only available in a subset of participants. 


\section{Statistical analyses}

All statistical analyses were performed with use of SPSS version 20.0 for Windows and version 23.0 for Mac (IBM SPSS, IBM Corp, Armonk, New York, USA). Fasting insulin concentrations and HOMA2-IR values were transformed with the natural logarithm prior to analyses because of non-normal distribution. A two-sided p-value $<0.05$ was considered statistically significant, except for interaction analyses, where the significance level was set at 0.10 .

Multiple linear regression analyses were performed to test whether insulin-related variables contributed to the variance in cognitive performance among individuals with type 2 diabetes. To this end, the unadjusted coefficient of determination, referred to as $R$-squared $\left(R^{2}\right)$ and reflecting explained variance, was used as effect estimate. More specifically, fasting insulin, C-peptide, and HOMA-IR were each combined with possible confounders in three subsequent regression models: model 1 demographic factors (ie, age, sex, and educational level); model 2 demographic factors and glycaemic control (ie, fasting glucose and $\mathrm{HbA1c}$ ); and model 3 demographic factors, glycaemic control, cardiovascular risk factors (ie, waist circumference, total/HDL-cholesterol ratio, use of lipid-modifying medication, systolic and diastolic blood pressure, use of antihypertensive medication, and smoking behaviour), and the presence of a depression. The incremental change in explained variance was assessed and statistically tested by the corresponding F-tests. In addition to changes in $\mathrm{R}^{2}$, we also report standardised regression coefficients that reflect the associations between insulin-related variables and cognitive performance.

We used multiplicative interaction terms to examine whether any association between insulin (resistance) and cognitive performance differed between individuals previously diagnosed with diabetes and those newly diagnosed at study entry, by gender, or by age. Non-linearity of the associations between fasting insulin or C-peptide and cognitive performance was explored by entering linear and quadratic terms of the mean-centered variables to the regression model, as at least fasting insulin concentrations have been shown to be quadratically associated with dementia risk in older individuals. ${ }^{14}$

As a sensitivity analysis, we re-examined the association between fasting insulin and cognitive performance after the exclusion of individuals treated with insulin. We also checked whether outliers influenced the results by excluding individuals with fasting insulin or C-peptide concentrations that were three standard deviations away from the sample mean. Finally, to test the robustness of our findings, analyses were rerun with adjustment for 24-hour ambulatory blood pressure instead of office blood pressure, as well as with additional adjustment for history of CVD. 
Table 6.1 Characteristics of the study population $(n=806)$

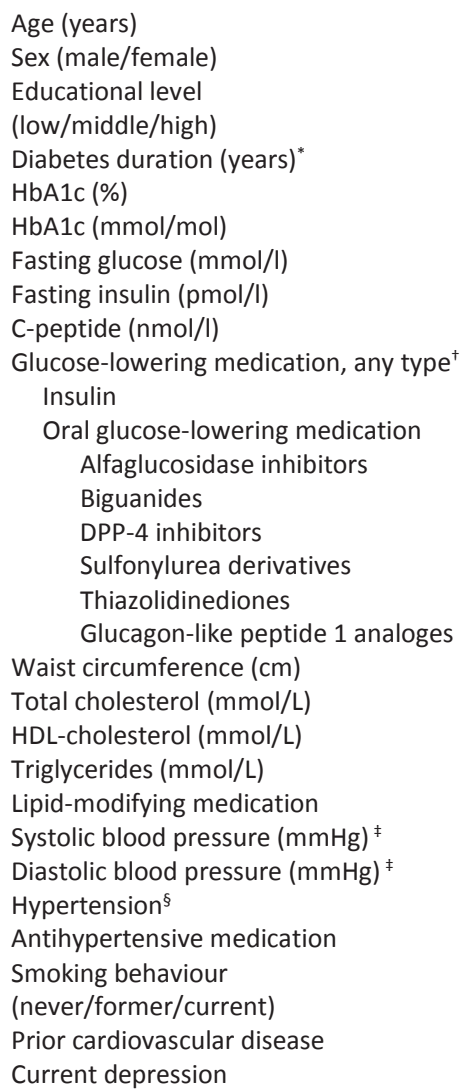

$62 \pm 8$

$542 / 264$

$215 / 357 / 234$

(26.7\%/44.3\%/29.0\%)

6 [3-12]

$6.9 \pm 1.1$

$52 \pm 12$

$7.9 \pm 2.0$

85 [53-134]

$0.85 \pm 0.43$

$628(91.4 \%)$

$165(24.0 \%)$

$591(86.0 \%)$

$0(0 \%)$

$562(81.8 \%)$

$51(7.4 \%)$

$168(24.5 \%)$

$8(1.2 \%)$

7 (1.0\%)

$105 \pm 13$

$4.5 \pm 1.0$

$1.3 \pm 0.4$

$1.5[1.1-2.1]$

$594(73.7 \%)$

$142 \pm 18$

$77 \pm 9$

$669(83.0 \%)$

577 (71.6\%)

$223 / 455 / 128$

$(27.7 \% / 56.5 \% / 15.9 \%)$

$218(27.0 \%)$

$76(9.4 \%)$

Data are presented as mean $\pm S D$, median [IQR], or $n(\%) .{ }^{*}$ Data available for $n=570$. ${ }^{\dagger}$ Data available for individuals with known type 2 diabetes $(n=687)$. ${ }^{\ddagger}$ Obtained from office blood pressure measurements. ${ }^{8}$ Hypertension was defined as a systolic blood pressure $\geq 140 \mathrm{mmHg}$ (based on office blood pressure measurements), a diastolic blood pressure $\geq 90 \mathrm{mmHg}$, and/or current use of antihypertensive medication. Abbreviations: DPP-4 = dipeptidyl peptidase-4.

\section{Results}

Of the first 975 individuals with type 2 diabetes who completed the baseline survey of The Maastricht Study, 114 (11.7\%) had no, or incomplete, data on measures of cognitive performance, and $22(2.3 \%)$ had missing data on fasting insulin or C-peptide concentrations. An additional 38 (3.9\%) individuals were excluded from the present analyses because of missing data on covariates, resulting in a final study population of 806 individuals. Differences in demographic, clinical, and diabetes-related characteristics 
between individuals with complete and incomplete data are depicted in Table S6.1 (Supplement). Notably, those excluded were generally older and less well educated.

Characteristics of the final study population are presented in Table 6.1. The mean age was $62 \pm 8$ years, $67.2 \%$ of the individuals were men, and $119(14.8 \%)$ were newly diagnosed with diabetes at study entry. Individuals with known type 2 diabetes had a median disease duration of 6 years and their overall glycaemic control was good with a mean $\mathrm{HbA} 1 \mathrm{c}$ of $7.0 \%$ (53 mmol/mol). Mean HbA1c concentrations were $6.2 \%$ (44 mmol/ $\mathrm{mol}$ ) in individuals with newly diagnosed diabetes.

\section{Fasting insulin, C-peptide, and cognitive performance}

Table 6.2 shows the variance in cognitive performance explained by fasting insulin and C-peptide concentrations above and beyond that explained by other factors (ie, demographics, glycaemic control, cardiovascular risk factors, and depression). Demographic factors alone (model 1 ) explained one-fifth to one-fourth of the variance in cognitive performance, with varying contributions across domains (ie, memory function (18.0\%), information processing speed (26.5\%), executive function and attention (22.8\%)). Fasting insulin or C-peptide were not associated with cognitive performance when added to this first model, nor did they increase the total explained variance of the model. Similar results were obtained when they were added to subsequent models with glycaemic control (model 2) and with cardiovascular risk factors and the presence of a depression (model 3). Specifically, across all models, the maximum increase in explained variance by either fasting insulin or C-peptide was $0.1 \%$ ( $p$-value for F-tests $\geq 0.48$ ). We did also not observe any quadratic association of insulin or C-peptide with cognitive performance (Table S6.2, Supplement).

Of note, incorporating measures of glycaemic control did not explain more variance in cognitive performance than did demographics alone, whereas the inclusion of cardiovascular risk factors and depression increased the explained variance with $1.1 \%$ to $2.4 \%$. This increase only reached statistical significance for information processing speed (p-value for F-tests $<0.01$ ).

\section{HOMA2-IR and cognitive performance}

Table 6.3 shows the variance in cognitive performance explained by HOMA2-IR as a marker of insulin resistance in non-insulin treated individuals $(n=641)$. Results for HOMA2-IR were roughly similar to those for insulin and C-peptide in that HOMA2-IR was not associated with cognitive performance and did not lead to an increase in the explained variance in cognition. 


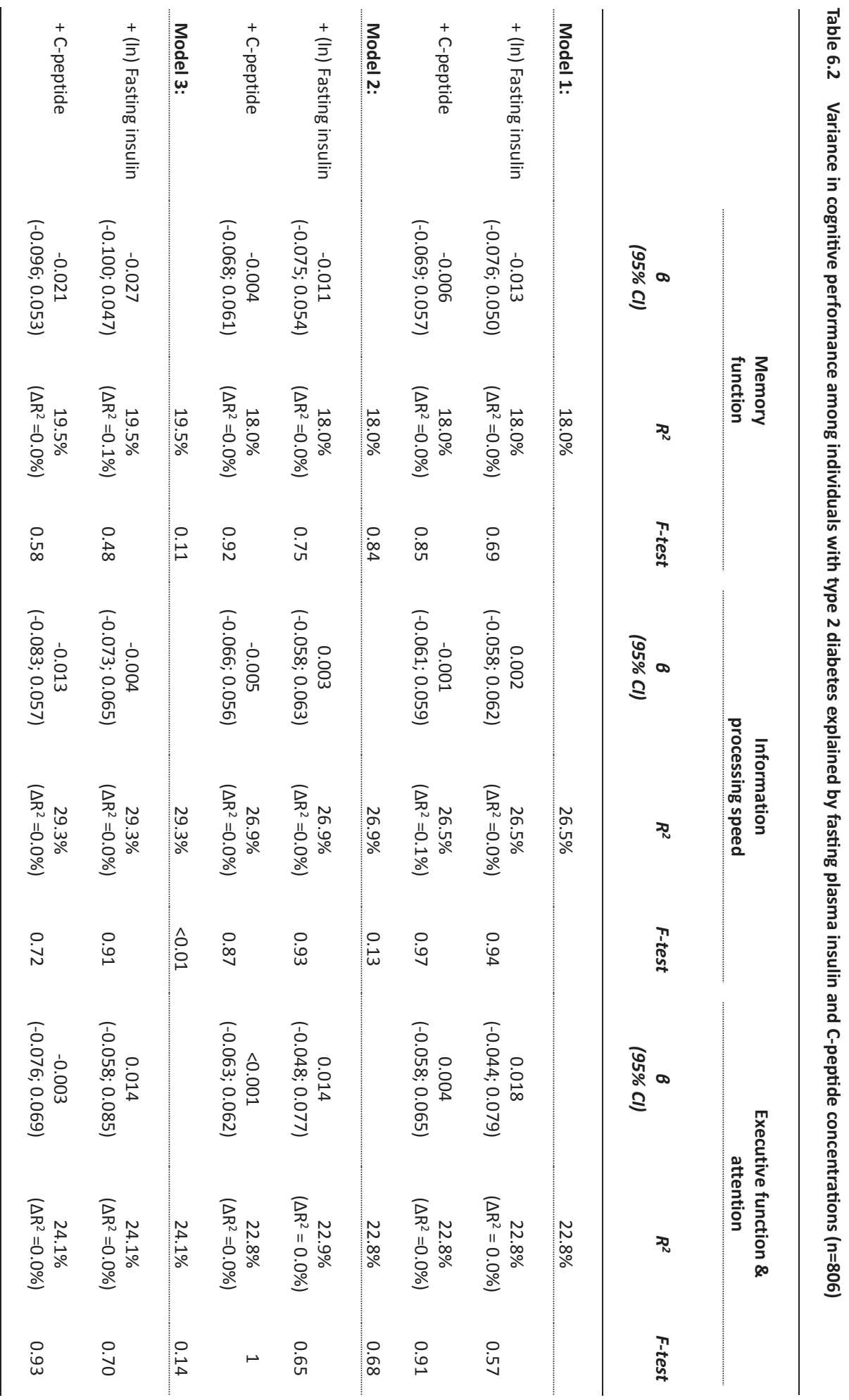




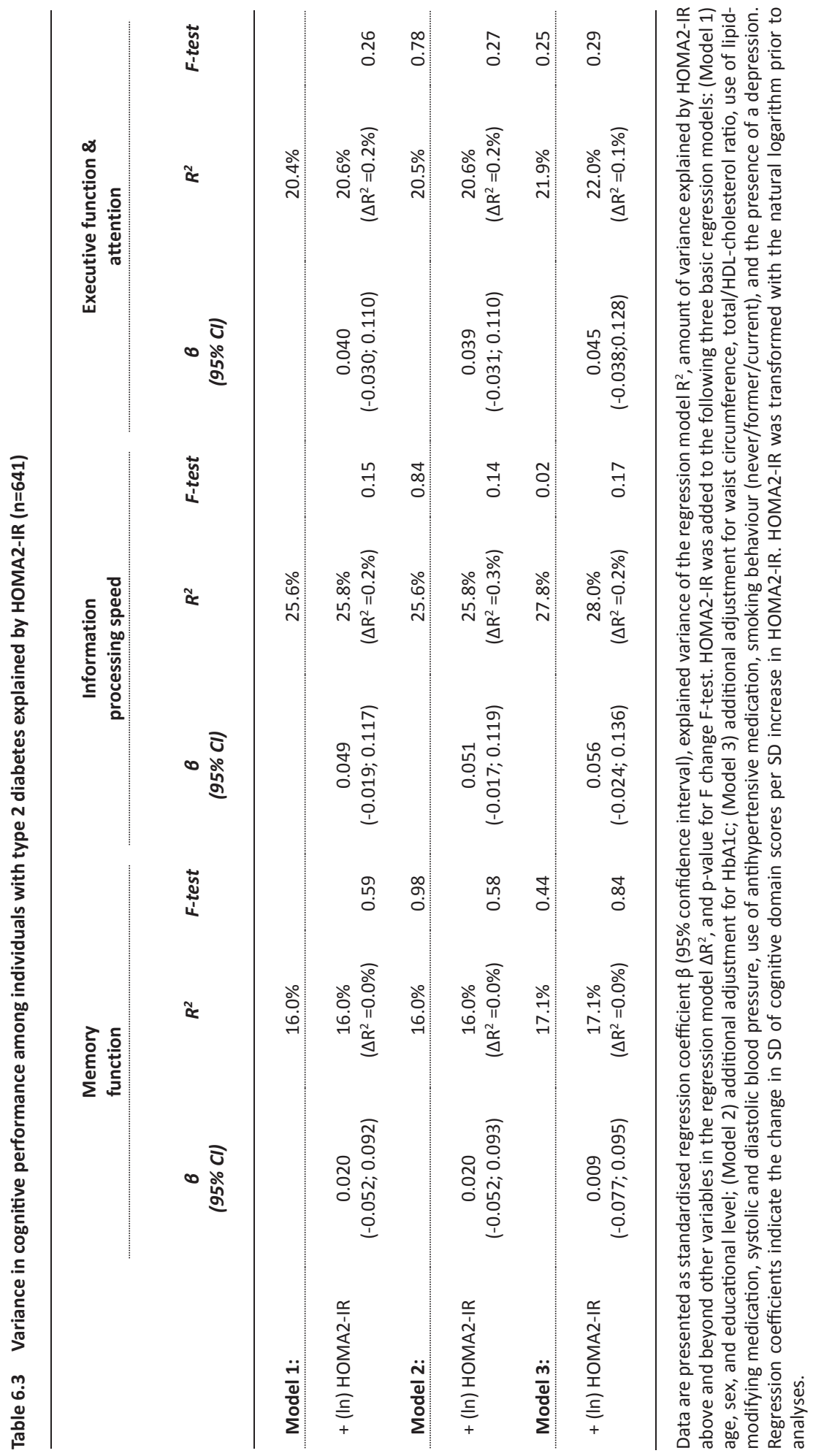


Legend to Table 6.2 (page 213)

Data are presented as standardised regression coefficient $\beta$ (95\% confidence interval), explained variance of the regression model $\mathrm{R}^{2}$, amount of variance explained by fasting insulin or $\mathrm{C}$-peptide concentrations above and beyond other variables in the regression model $\Delta R^{2}$, and $p$-value for $F$ change $F$-test. Fasting insulin and C-peptide concentrations were each added to the following three basic regression models: (Model 1) age, sex, and educational level; (Model 2) additional adjustment for HbA1c, and fasting glucose; (Model 3) additional adjustment for waist circumference, total/HDL-cholesterol ratio, use of lipid-modifying medication, systolic and diastolic blood pressure, use of antihypertensive medication, smoking behaviour (never/former/current), and the presence of a depression. Regression coefficients indicate the change in SD of cognitive domain scores per SD increase in fasting insulin or C-peptide concentrations. Fasting insulin concentrations were transformed with the natural logarithm prior to analyses.

\section{Interaction analyses}

Interaction analyses showed that age and sex did not clearly modify the null-association of insulin-related variables with cognitive performance (data not shown). Likewise, results were similar between individuals with previously and newly diagnosed diabetes (data not shown).

\section{Sensitivity analyses}

Because the use of exogenous insulin may affect the association of insulin concentrations with cognitive performance, we reran the analysis in the subgroup of individuals not treated with insulin ( $n=641)$. In this subgroup, the association between fasting insulin and cognitive performance was also non-significant (Table S6.3, Supplement). Likewise, after exclusion of individuals with extreme values of fasting insulin $(n=6), C$-peptide $(n=9)$, or HOMA2-IR $(n=1)$, the results remained virtually unchanged (data not shown). Alternative adjustment for 24-hour blood pressure and additional adjustment for a history of CVD did also not materially alter our results (data not shown).

\section{Conclusions}

The present study shows that neither HOMA2-IR nor fasting plasma insulin or C-peptide concentrations are associated with cognitive performance among individuals in late middle age with adequately controlled type 2 diabetes.

To our knowledge, this is the largest and most comprehensive study to date examining the association between insulin (resistance) and cognitive performance among people with type 2 diabetes. Our results reinforce those of previous smaller studies showing that insulin-related variables are unrelated to cognitive performance ${ }^{15-19}$ and cognitive decline $^{18,20}$ among individuals with diabetes, even in those with less well-controlled diabetes. ${ }^{17}$ A few other small studies, ${ }^{21-28}$ some of which evaluated overlapping populations, ${ }^{23-25}$ have reported seemingly contradictory results, but these studies were often conducted in selected populations, for example focusing on individuals with 
mild cognitive impairment, ${ }^{21,22}$ and did not always adequately control for demographic factors. ${ }^{21,22,26,27}$ Collectively, the currently available data thus strongly suggest that, in a population-based sample of individuals with type 2 diabetes, measures of peripheral insulin resistance are unrelated to cognitive performance.

The lack of an association between systemic insulin resistance and cognitive performance in type 2 diabetes might be somewhat surprising given that such an association has been established in (middle-aged) individuals without diabetes $\left(\mathrm{eg},{ }^{4-6}\right)$. The association of insulin (resistance) with cognitive performance might thus differ by diabetes status, which is supported by two previous studies showing that fasting hyperinsulinaemia ${ }^{29}$ and higher HOMA values ${ }^{19}$ are associated with worse cognitive performance ${ }^{19}$ and an increased risk for dementia ${ }^{29}$ among individuals without diabetes, but not in those with diabetes. Additional analyses in the Maastricht Study cohort also revealed a trend towards worse memory function with increasing insulin (resistance) among individuals with a normal glucose tolerance ( $n=1,773$, mean age $58 \pm 8$ years, $43 \%$ men), albeit not independent of vascular factors (data not shown). One potential explanation for this discrepancy lies in the receptor-mediated transport of insulin across the blood-brain barrier, which has been shown to decrease with the degree of insulin resistance. ${ }^{30}$ As a consequence, peripheral insulin may not accurately reflect central (ie, cerebral) concentrations of insulin in those with diabetes. Alternatively, however, such a discrepancy could also relate to the potential inaccuracy of fasting insulin and HOMA2IR as measures of (peripheral) insulin resistance in diabetes, not only because $\beta$-cells often lose their insulin secretion capacity as the disease progresses, but also because oral glucose-lowering medication can enhance insulin secretion. ${ }^{31}$

In a broader context, it is possible that the factors that contribute to cognitive dysfunction among individuals with type 2 diabetes differ from those in the general population. Noteworthy in this regard is our observation that traditional cardiovascular risk factors explained only a marginal amount of variance (ie, $1.1 \%$ to $2.4 \%$ ) in cognitive performance beyond that explained by demographics, even when we used the 24-hour blood pressure data. Although cardiovascular risk factors, such as obesity, hypertension, and dyslipidemia, may be associated with an increased risk of cognitive dysfunction in the general population, ${ }^{32,33}$ a recent review concluded that it remains to be determined whether these factors are also linked to cognitive performance among individuals with type 2 diabetes. ${ }^{34}$ It is conceivable that the earlier detection and more intensive treatment of cardiovascular risk factors in individuals diagnosed with diabetes might limit the potential detrimental effects these factors can have on the brain. Likewise, it is possible that multiple cardiovascular risk factors do not necessarily exert additive effects on the brain. Nevertheless, the relatively small proportion of variance explained by traditional 
cardiovascular risk factors in our study does not undoubtedly mean that these factors are irrelevant to cognitive performance in diabetes. Previous studies have, for example, suggested that cardiovascular risk factors in midlife are more closely associated with late-life than with midlife cognitive performance. ${ }^{35}$ In addition, risk factors such as blood pressure might be non-linearly related to cognitive performance among individuals with type 2 diabetes. ${ }^{36}$

Although we can conclude from our data that measures of peripheral insulin resistance are unrelated to cognitive performance in type 2 diabetes, we cannot preclude the possibility that central insulin resistance may affect cognitive performance. This is not only because peripheral insulin concentrations may not adequately reflect cerebral concentrations, as mentioned above, but also because of the possibility of peripheral insulin resistance being not invariably accompanied by central insulin resistance. ${ }^{37}$ Two recent pilot studies in a small sample of older individuals with type 2 diabetes and agematched healthy individuals have demonstrated that a single dose of intranasal insulin can acutely improve visuospatial memory performance, ${ }^{38,39}$ highlighting the effects cerebral insulin may have on the brain in diabetes. Experimental studies have identified multiple pathways through which insulin signalling may affect cognitive performance. It, for example, affects synaptic plasticity, modulates neurotransmitter release, and contributes to the glucose availability in the brain. ${ }^{7}$ At the same time, insulin is known to interact with the metabolism of amyloid- $\beta$ and tau, ${ }^{7}$ key proteins in the pathogenesis of Alzheimer's disease. Unfortunately, however, it is currently not possible to measure insulin signaling in the human brain in vivo. Consequently, the role of central insulin signaling in cognitive performance remains elusive.

Strengths of the present study include its relatively large sample size and the use of a comprehensive neuropsychological test battery to assess cognitive performance across multiple domains. The findings discussed above should, however, also be viewed in the light of a few limitations. Most importantly, our study population was characterized by a relatively large proportion of highly-educated (29\%) individuals, a relatively young age (mean age $62 \pm 8$ years), and overall adequate glycaemic control (mean $\mathrm{HbA1c}$ $6.9 \pm 1.1 \%(52 \pm 12 \mathrm{mmol} / \mathrm{mol}))$. This seemed partly enhanced by our complete case analysis approach (eg, for educational level and age, Table S6.1 (Supplement)), and may not only have resulted in slightly underestimated effects, but also somewhat limits the generalisability of our findings. Another limitation might be that the venous blood sampling and cognitive assessment were not performed at the same day. Consequently, we may have missed acute effects of hyperinsulinaemia due to intraindividual day-today variability in insulin resistance. ${ }^{40}$ We believe, however, that these limitations are unlikely to fully explain the null-associations observed in the present study. 


\section{Chapter 6}

In conclusion, the present study shows that peripheral hyperinsulinaemia and insulin resistance are unrelated to cognitive performance among individuals with type 2 diabetes. Our results therefore indicate that measures of peripheral insulin resistance are unlikely to explain the variety of cognitive problems among individuals with diabetes. Further studies are warranted to elucidate the effects of central insulin (resistance) on cognition in diabetes and to adequately address the possibility of central and peripheral insulin (resistance) being differentially associated with cognitive performance. 


\section{References}

1. Koekkoek, P.S., et al., Cognitive function in patients with diabetes mellitus: guidance for daily care. Lancet Neurol, 2015. 14(3): p. 329-40.

2. Lutgers, H.L., et al., Life expectancy in a large cohort of type 2 diabetes patients treated in primary care (ZODIAC-10). PLoS One, 2009. 4(8): p. e6817.

3. Geijselaers, S.L., et al., Glucose regulation, cognition, and brain MRI in type 2 diabetes: a systematic review. Lancet Diabetes Endocrinol, 2015. 3(1): p. 75-89.

4. Young, S.E., A.G. Mainous, 3rd, and M. Carnemolla, Hyperinsulinemia and cognitive decline in a middle-aged cohort. Diabetes Care, 2006. 29(12): p. 2688-93.

5. Okereke, O., et al., Plasma C peptide level and cognitive function among older women without diabetes mellitus. Arch Intern Med, 2005. 165(14): p. 1651-6.

6. Okereke, O.I., et al., Fasting plasma insulin, C-peptide and cognitive change in older men without diabetes: results from the Physicians' Health Study II. Neuroepidemiology, 2010. 34(4): p. 200-7.

7. Cholerton, B., L.D. Baker, and S. Craft, Insulin, cognition, and dementia. Eur J Pharmacol, 2013. 719(1-3): p. 170-9.

8. Schram, M.T., et al., The Maastricht Study: an extensive phenotyping study on determinants of type 2 diabetes, its complications and its comorbidities. Eur J Epidemiol, 2014. 29(6): p. 439-51.

9. World Health Organisation. Definition and diagnosis of diabetes mellitus and intermediate hyperglycemia: report of a WHO/IDF consultation. 2006. Available from: http://www.idf.org/ webdata/docs/WHO_IDF_definition_diagnosis_of_diabetes.pdf. Last accessed July 19, 2015.

10. Horwitz, D.L., et al., Proinsulin, insulin, and C-peptide concentrations in human portal and peripheral blood. J Clin Invest, 1975. 55(6): p. 1278-83.

11. Sima, A.A. and H. Kamiya, Is C-peptide replacement the missing link for successful treatment of neurological complications in type 1 diabetes? Curr Drug Targets, 2008. 9(1): p. 37-46.

12. Wallace, T.M., J.C. Levy, and D.R. Matthews, Use and abuse of HOMA modeling. Diabetes Care, 2004. 27(6): p. 1487-95.

13. Spauwen, P.J., et al., Associations of advanced glycation end-products with cognitive functions in individuals with and without type 2 diabetes: the maastricht study. J Clin Endocrinol Metab, 2015. 100(3): p. 951-60.

14. Peila, R., et al., Fasting insulin and incident dementia in an elderly population of JapaneseAmerican men. Neurology, 2004. 63(2): p. 228-33.

15. Manschot, S.M., et al., Metabolic and vascular determinants of impaired cognitive performance and abnormalities on brain magnetic resonance imaging in patients with type 2 diabetes. Diabetologia, 2007. 50(11): p. 2388-97.

16. Tiehuis, A.M., et al., Cognitive dysfunction and its clinical and radiological determinants in patients with symptomatic arterial disease and diabetes. J Neurol Sci, 2009. 283(1-2): p. 1704.

17. Akisaki, T., et al., Cognitive dysfunction associates with white matter hyperintensities and subcortical atrophy on magnetic resonance imaging of the elderly diabetes mellitus Japanese elderly diabetes intervention trial (J-EDIT). Diabetes Metab Res Rev, 2006. 22(5): p. 376-84.

18. Imamine, R., et al., Does cerebral small vessel disease predict future decline of cognitive function in elderly people with type 2 diabetes? Diabetes Res Clin Pract, 2011. 94(1): p. 91-9.

19. Benedict, C., et al., Impaired insulin sensitivity as indexed by the HOMA score is associated with deficits in verbal fluency and temporal lobe gray matter volume in the elderly. Diabetes Care, 2012. 35(3): p. 488-94.

20. Reijmer, Y.D., et al., Accelerated cognitive decline in patients with type 2 diabetes: MRI correlates and risk factors. Diabetes Metab Res Rev, 2011. 27(2): p. 195-202.

21. Gao, Y., et al., The characteristic of cognitive function in Type 2 diabetes mellitus. Diabetes 


\section{Chapter 6}

Res Clin Pract, 2015.

22. Abbatecola, A.M., et al., Rosiglitazone and cognitive stability in older individuals with type 2 diabetes and mild cognitive impairment. Diabetes Care, 2010. 33(8): p. 1706-11.

23. Umegaki, H., et al., Factors associated with cognitive decline in elderly diabetics. Dement Geriatr Cogn Dis Extra, 2011. 1(1): p. 1-9.

24. Umegaki, H., et al., Factors associated with cognitive decline in older adults with type 2 diabetes mellitus during a 6-year observation. Geriatr Gerontol Int, 2015. 15(3): p. 302-10.

25. Umegaki, H., et al., Glucose control levels, ischaemic brain lesions, and hyperinsulinaemia were associated with cognitive dysfunction in diabetic elderly. Age Ageing, 2008. 37(4): p. 458-61.

26. Yanagawa, M., et al., Association between improvements in insulin resistance and changes in cognitive function in elderly diabetic patients with normal cognitive function. Geriatr Gerontol Int, 2011. 11(3): p. 341-7.

27. Suzuki, M., et al., Association between insulin resistance and cognitive function in elderly diabetic patients. Geriatrics \& Gerontology International, 2006. 6(4): p. 254-259.

28. Pearce, K.L., et al., Continuous glucose monitoring and cognitive performance in type 2 diabetes. Diabetes Technol Ther, 2012. 14(12): p. 1126-33.

29. Luchsinger, J.A., et al., Hyperinsulinemia and risk of Alzheimer disease. Neurology, 2004. 63(7): p. 1187-92.

30. Kern, W., et al., Low cerebrospinal fluid insulin levels in obese humans. Diabetologia, 2006. 49(11): p. 2790-2.

31. DeFronzo, R.A., Pharmacologic therapy for type 2 diabetes mellitus. Ann Intern Med, 2000. 133(1): p. 73-4.

32. Plassman, B.L., et al., Systematic review: factors associated with risk for and possible prevention of cognitive decline in later life. Ann Intern Med, 2010. 153(3): p. 182-93.

33. Van den Berg, E., et al., Type 2 diabetes mellitus, hypertension, dyslipidemia and obesity: A systematic comparison of their impact on cognition. Biochim Biophys Acta, 2009. 1792(5): p. 470-81.

34. Feinkohl, I., et al., The impact of diabetes on cognitive decline: potential vascular, metabolic, and psychosocial risk factors. Alzheimers Res Ther, 2015. 7(1): p. 46.

35. Reijmer, Y.D., et al., Development of vascular risk factors over 15 years in relation to cognition: the Hoorn Study. J Am Geriatr Soc, 2012. 60(8): p. 1426-33.

36. Spauwen, P.J., et al., Both Low and High 24-Hour Diastolic Blood Pressure Are Associated With Worse Cognitive Performance in Type 2 Diabetes: The Maastricht Study. Diabetes Care, 2015. 38(8): p. 1473-80.

37. Banks, W.A., J.B. Owen, and M.A. Erickson, Insulin in the brain: there and back again. Pharmacol Ther, 2012. 136(1): p. 82-93.

38. Novak, V., et al., Enhancement of vasoreactivity and cognition by intranasal insulin in type 2 diabetes. Diabetes Care, 2014. 37(3): p. 751-9.

39. Zhang, H., et al., Intranasal insulin enhanced resting-state functional connectivity of hippocampal regions in type 2 diabetes. Diabetes, 2015. 64(3): p. 1025-34.

40. Jayagopal, V., et al., Biological variation of homeostasis model assessment-derived insulin resistance in type 2 diabetes. Diabetes Care, 2002. 25(11): p. 2022-5. 
Insulin resistance and cognition in diabetes 


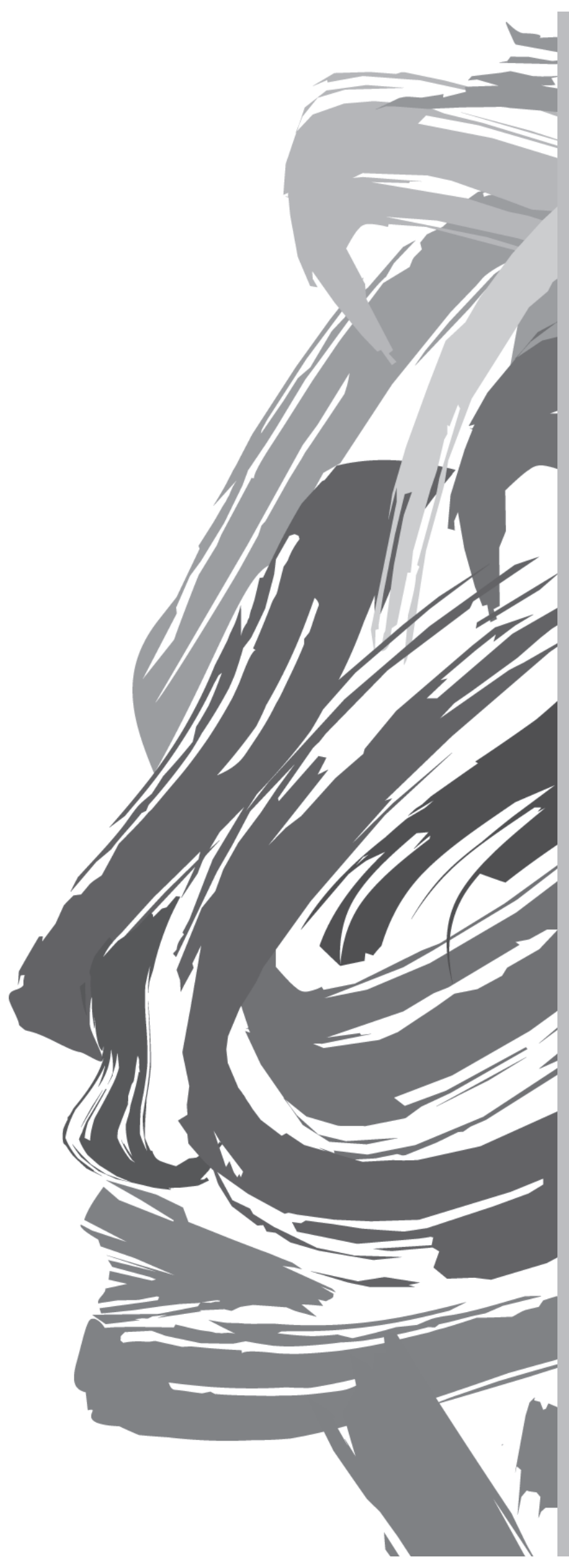


Supplement to Chapter 6 


\section{Extended research design and methods}

\section{Cognitive performance}

The composite memory score was derived from the Verbal Learning Test by weighting total immediate and delayed recall scores. The domain processing speed included the Stroop Colour Word Test Part I and II, the Concept Shifting Test Part A and B, and the Letter-Digit Substitution Test. Executive function and attention was assessed by the Stroop Colour Word Test Part III and the Concept Shifting Test Part C. A brief description of the individual tests is provided below.

Raw test scores were transformed into z-scores. Standardised scores of the Stroop Colour Word Test and Concept Shifting Test were inverted so that higher scores indicated better cognitive performance. Thereafter, domain-specific scores were calculated by averaging the $z$-scores from (sub)tests within that domain (eg, memory $=z$-score ${ }_{\text {immediate }}$ recall $+z$-score delayed recall $/ 2$ ).

Description of the individual cognitive tests used in the present study:

\section{Verbal Learning Test $^{1}$}

Fifteen unrelated, monosyllabic, words were presented on a computer screen in five subsequent trials. After each trial, participants were instructed to recall as many words as possible in any order. Twenty minutes after the last trial, participants were asked again to reproduce the words. Outcomes recorded included the total number of words correctly recalled over the five trials (total immediate recall) and the number of correctly recalled words during delayed recall (delayed recall).

\section{Stroop Colour Word Test ${ }^{2}$}

In this test, that consisted of three parts, participants were firstly asked to read aloud color names (ie, red, blue, yellow, and green) that were printed in black ink (Part I). Secondly, they were instructed to name solid color patches (Part II). Finally, participants had to name the ink color of color names that were printed in an incongruent color (eg, participants were asked to say red when the word yellow was printed in red) (Part III). The time needed to complete Part III was adjusted for the average time needed to complete Part I and II.

\section{Concept Shifting Test ${ }^{3}$}

This test, a modification of the Trailing Making Test, consisted of four subtasks. During each subtask, participants were shown 16 small circles aligned along a larger imaginary circle. The small circles contained (a combination of) digits, letters, or were empty. 
Participants were instructed to cross-out as quickly as possible the digits in ascending order (Part A), the letters in alphabetic order (Part B), and the letters and digits in alternating order (Part C). Thereafter, participants were asked to cross-out empty circles in a clockwise fashion in two consecutive trials (Part 0 ). In this way, test results could be accounted for basic motor speed. The time needed to complete subtasks A and B was adjusted for the average time needed to complete Part 0 , the time needed to complete Part $\mathrm{C}$ for the average time of Part $\mathrm{A}$ and $\mathrm{B}$.

\section{Letter-Digit Substitution Test ${ }^{4}$}

Participants were requested to match digits to letters according to a given key. This key included the numbers 1 to 9, each paired with a different letter. The outcome of interest was the number of correct substitutions within 90 seconds. 


\section{References}

1. Van der Elst, W., et al., Rey's verbal learning test: normative data for 1855 healthy participants aged 24-81 years and the influence of age, sex, education, and mode of presentation. J Int Neuropsychol Soc, 2005. 11(3): p. 290-302.

2. Van der Elst, W., et al., The Stroop color-word test: influence of age, sex, and education; and normative data for a large sample across the adult age range. Assessment, 2006. 13(1): p. 6279.

3. Van der Elst, W., et al., The Concept Shifting Test: adult normative data. Psychol Assess, 2006. 18(4): p. 424-32.

4. Van der Elst, W., et al., The Letter Digit Substitution Test: normative data for 1,858 healthy participants aged 24-81 from the Maastricht Aging Study (MAAS): influence of age, education, and sex. J Clin Exp Neuropsychol, 2006. 28(6): p. 998-1009. 
Table S6.1 Characteristics of individuals with complete and incomplete data in the present study

\begin{tabular}{|c|c|c|c|c|}
\hline & $\begin{array}{c}\text { Included } \\
(n=806)\end{array}$ & $\begin{array}{c}\text { Excluded } \\
(n=169)\end{array}$ & Missing* & $\begin{array}{c}\text { p- } \\
\text { value }^{+}\end{array}$ \\
\hline Age (years) & $62 \pm 8$ & $64 \pm 8$ & 0 & 0.003 \\
\hline Sex (male/female) & $542 / 264$ & $118 / 51$ & 0 & 0.515 \\
\hline Educational level & $215 / 357 / 234$ & $55 / 48 / 20$ & 46 & $<0.001$ \\
\hline (low/middle/high) & $(26.7 \% / 44.3 \% / 29.0 \%)$ & $(32.5 \% / 28.4 \% / 11.8 \%)$ & & \\
\hline $\mathrm{HbA1c}(\%)$ & $6.9 \pm 1.1$ & $7.0 \pm 1.1$ & 3 & 0.49 \\
\hline $\mathrm{HbA} 1 \mathrm{c}(\mathrm{mmol} / \mathrm{mol})$ & $52 \pm 12$ & $53 \pm 12$ & 3 & 0.52 \\
\hline Fasting glucose (mmol/L) & $7.9 \pm 2.0$ & $7.8 \pm 1.9$ & 1 & 0.269 \\
\hline Fasting insulin (pmol/L) & $86[53-134]$ & $86[50-138]$ & 22 & 0.825 \\
\hline Fasting C-peptide (nmol/L) & $0.85 \pm 0.43$ & $0.88 \pm 0.51$ & 17 & 0.602 \\
\hline Glucose-lowering medication, any type & $628(77.9 \%)$ & $137(81.1 \%)$ & 1 & 0.297 \\
\hline Insulin & $165(20.5 \%)$ & $51(30.2 \%)$ & 1 & 0.005 \\
\hline Oral glucose-lowering medication & $591(73.3 \%)$ & $122(72.2 \%)$ & 1 & 0.851 \\
\hline Waist circumference $(\mathrm{cm})$ & $105 \pm 13$ & $108 \pm 14$ & 2 & 0.027 \\
\hline Total cholesterol (mmol/L) & $4.5 \pm 1.0$ & $4.3 \pm 1.0$ & 1 & 0.126 \\
\hline HDL-cholesterol (mmol/L) & $1.3 \pm 0.4$ & $1.3 \pm 0.3$ & 1 & 0.964 \\
\hline Triglycerides (mmol/L) & $1.5[1.1-2.1]$ & $1.6[1.2-2.1]$ & 1 & 0.945 \\
\hline Lipid lowering medication & $594(73.7 \%)$ & $132(78.1 \%)$ & 1 & 0.187 \\
\hline Systolic blood pressure $(\mathrm{mmHg})^{\ddagger}$ & $142 \pm 18$ & $144 \pm 19$ & 0 & 0.312 \\
\hline Diastolic blood pressure $(\mathrm{mmHg})^{\ddagger}$ & $77 \pm 9$ & $77 \pm 10$ & 0 & 0.298 \\
\hline Hypertension ${ }^{\S}$ & $669(83.0 \%)$ & $142(84.0 \%)$ & 1 & 0.631 \\
\hline Antihypertensive medication & $577(71.6 \%)$ & $125(74.0 \%)$ & 1 & 0.459 \\
\hline Smoking behaviour & $223 / 455 / 128$ & $45 / 68 / 23$ & 33 & 0.341 \\
\hline (never/former/current) & $(27.7 \% / 56.5 \% / 15.9 \%)$ & $(26.6 \% / 40.2 \% / 13.6 \%)$ & & \\
\hline Current depression & $76(9.4 \%)$ & $7(4.1 \%)$ & 57 & 0.272 \\
\hline Memory & $0.05 \pm 0.94$ & $-0.37 \pm 0.97$ & 65 & $<0.001$ \\
\hline Processing speed & $0.05 \pm 0.77$ & $-0.16 \pm 0.82$ & 93 & 0.026 \\
\hline Executive function \& attention & $0.04 \pm 0.80$ & $-0.19 \pm 0.96$ & 101 & 0.023 \\
\hline
\end{tabular}

Data are presented as mean $\pm S D$, median [IQR], or $n(\%)$. ${ }^{*}$ Number of missings for a specific variable. ${ }^{\dagger} \mathrm{P}$-value for difference between individuals with and without complete data as evaluated with use of independent samples t-tests for continuous variables and Chi-square tests for categorical variables. ${ }^{\ddagger}$ Obtained from office blood pressure measurements. ${ }^{\S}$ Hypertension was defined as a systolic blood pressure $\geq 140 \mathrm{mmHg}$ (based on at least three office blood pressure measurements), a diastolic blood pressure $\geq 90 \mathrm{mmHg}$, and/or current use of antihypertensive medication. Abbreviations: $\mathrm{HDL}=$ high density lipoprotein. 
Table S6.2 Quadratic associations between fasting insulin or C-peptide concentrations and cognitive performance

\begin{tabular}{lccc}
\hline & $\begin{array}{c}\text { Memory } \\
\text { function }\end{array}$ & $\begin{array}{c}\text { Information } \\
\text { Processing speed }\end{array}$ & $\begin{array}{c}\text { Executive function } \\
\text { and attention }\end{array}$ \\
\hline Insulin & -0.007 & 0.007 & 0.017 \\
Insulin & $(-0.074$ to 0.060$)$ & $(-0.057$ to 0.070$)$ & $(-0.048$ to 0.082$)$ \\
& 0.018 & 0.013 & -0.003 \\
C-peptide & $\mathrm{P}=0.604$ & $\mathrm{P}=0.686$ & $\mathrm{P}=0.929$ \\
& -0.021 & & -0.006 \\
C-peptide & $(-0.093$ to 0.051$)$ & 0.003 & $(-0.077$ to 0.064$)$ \\
& 0.030 & $(-0.066$ to 0.071$)$ & 0.021 \\
& $\mathrm{P}=0.408$ & -0.008 & $\mathrm{P}=0.559$ \\
\hline
\end{tabular}

$\mathrm{N}=806$. Data are presented as standardised regression coefficients ( $95 \% \mathrm{Cl}$ or p-value). Fasting plasma insulin concentrations were transformed with the natural logarithm before analyses. Both (In) insulin and C-peptides were centered around the mean before being squared to reduce the possibility of multicollinearity.

Data are presented as standardised regression coefficient $\beta$ (95\% confidence interval), explained variance of the regression model $R^{2}$, amount of variance explained by fasting insulin or $C$-peptide concentrations above and beyond other variables in the regression model $\Delta R^{2}$, and $p$-value for $F$ change F-test. Fasting insulin and C-peptide concentrations were each added to the following three basic regression models: (Model 1) age, sex, and educational level; (Model 2) additional adjustment for HbA1c, and fasting glucose; (Model 3) additional adjustment for waist circumference, total/HDL-cholesterol ratio, use of lipid-modifying medication, systolic and diastolic blood pressure, use of antihypertensive medication, smoking behaviour (never/former/current), and the presence of a depression. Regression coefficients indicate the change in SD of cognitive domain scores per SD increase in fasting insulin or C-peptide concentrations. Fasting insulin concentrations were transformed with the natural logarithm prior to analyses. 


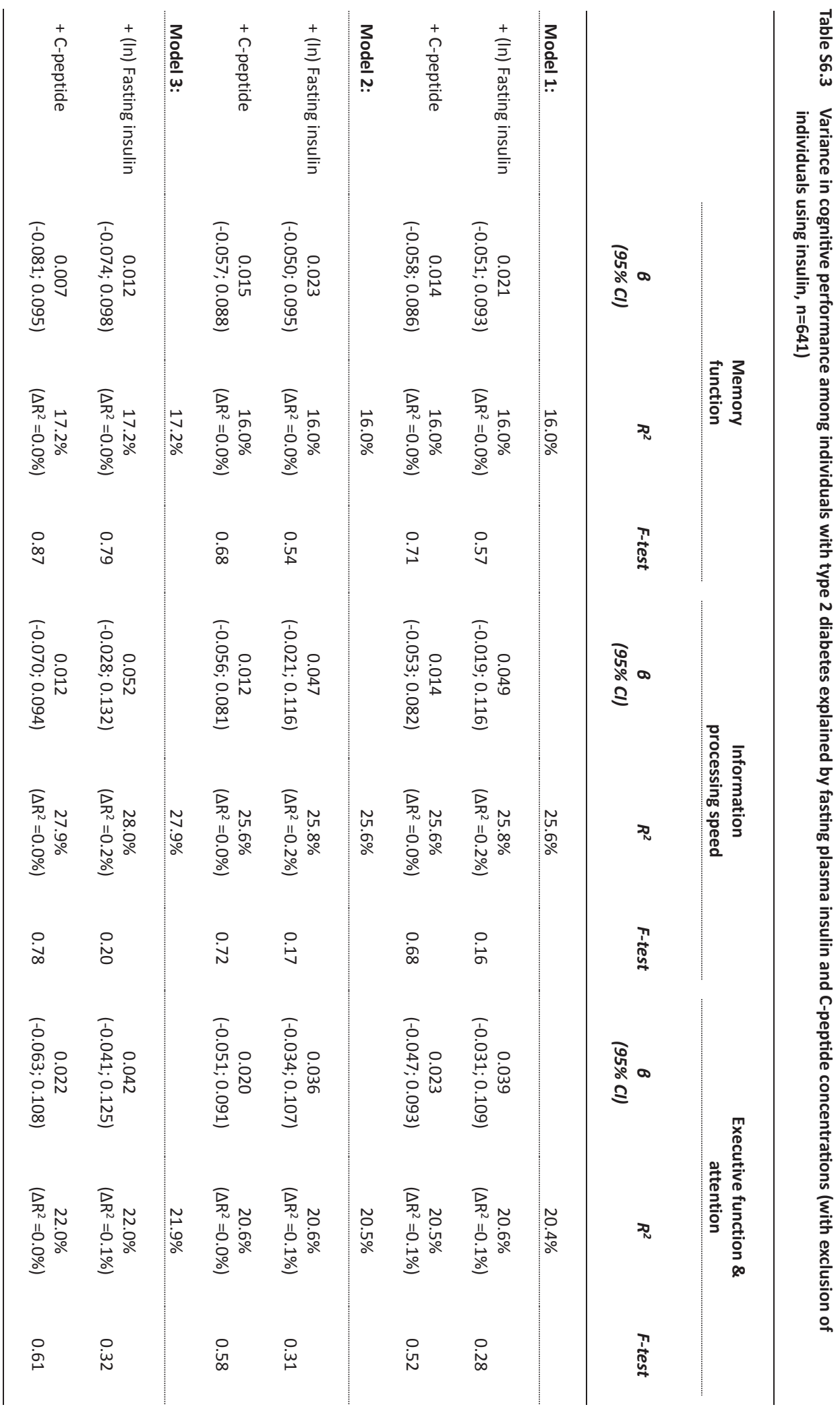




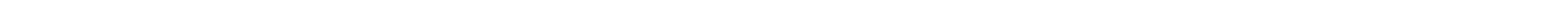




\section{Chapter 7}

\section{ASSOCIATION OF CEREBROSPINAL FLUID INSULIN WITH COGNITIVE PERFORMANCE AND CEREBROSPINAL FLUID BIOMARKERS OF ALZHEIMER'S DISEASE:}

THE PEARL NEURODEGENERATIVE DISEASES

Stefan L.C. Geijselaers, Coen D.A. Stehouwer, Geert Jan Biessels On behalf of the Pearl Neurodegenerative Diseases Study Group 


\section{Chapter 7}

\section{Abstract}

\section{Objective}

Abnormal insulin signalling in the brain has been linked to Alzheimer's disease. Here, we evaluated whether insulin levels in the cerebrospinal fluid (CSF) are associated with cognitive performance and CSF biomarkers of Alzheimer's disease among patients referred to academic memory clinics.

\section{Methods}

This cross-sectional study included 138 individuals (mean age $66 \pm 9$ years, $65.2 \%$ male) diagnosed with either subjective cognitive impairment $(n=45)$, amnestic mild cognitive impairment $(n=44)$, or Alzheimer's disease ( $n=49$ ), who completed a neuropsychological assessment, including tests of global cognition and memory performance, and who underwent lumbar puncture to measure CSF levels of insulin, amyloid- $\beta$, tau, and phosphorylated ( $p$-)tau.

\section{Results}

CSF insulin did not differ between the diagnostic groups $(p=0.136)$. Likewise, after adjustment for age, sex, body mass index, diabetes status, and clinic site, CSF insulin was unrelated to cognitive performance and biomarkers of Alzheimer's disease across the whole population (all $p \geq 0.131$ ). Importantly, however, effect modification by sex and APOE- $\varepsilon 4$ genotype was observed in that higher CSF insulin was associated with worse global cognition (standardised regression coefficient $-0.483 ; p=0.008)$ and higher $p$-tau levels $(0.353 ; p=0.040)$ in women and with higher tau $(0.287$; $p=0.008)$ and $p$-tau (0.246; $p=0.029)$ among non-carriers of the APOE- $\varepsilon 4$ allele.

\section{Conclusions}

Our findings not only provide further evidence linking brain insulin signalling to Alzheimer's disease, but also highlight the need to consider sex and APOE- $\varepsilon 4$ genotype when assessing the role of insulin. 


\section{Introduction}

Over the last decades, a growing body of evidence, derived from in vitro experiments, animal models, and observations in patients, has linked abnormal insulin signalling to Alzheimer's disease (AD). ${ }^{1-4}$ Multiple pathways may account for this, which have been extensively reviewed elsewhere. ${ }^{1-4}$ Insulin is, for example, believed to interfere with the metabolism of amyloid- $\beta(A \beta)$ and tau. ${ }^{3}$

At present, brain insulin signalling cannot be measured directly in humans in vivo. Evidence of deficient insulin signalling in patients with AD therefore stems from postmortem brain studies showing fewer insulin receptors and decreased downstream signalling activity in the brains of individuals with AD as compared to age-matched controls. ${ }^{5}$ To overcome the lack of direct measures, previous clinical and epidemiological studies exploring cerebral effects of insulin have generally used markers of peripheral insulin resistance as a possible indicator of deficient brain signalling. However, peripheral insulin levels and insulin sensitivity may not accurately reflect the cerebral situation. ${ }^{6}$ For example, transport of insulin across the blood-brain barrier decreases with increasing insulin resistance, ${ }^{7}$ and there even appears to be local production of insulin in the brain. ${ }^{8}$ Insulin levels in the cerebrospinal fluid (CSF) likely more accurately reflect insulin levels within the central nervous system, and hence could be useful to assess the effects of insulin exposure on the brain. However, published data on CSF insulin in relation to AD are scarce and inconclusive. ${ }^{9-13}$ We therefore evaluated, in a memory clinic population, the associations of CSF insulin with cognitive performance and CSF biomarkers of AD (ie, $A \beta$, tau, and phosphorylated tau ( $p$-tau)).

\section{Methods}

\section{Study population}

This study was based on data from the Pearl Neurodegenerative Diseases, a collaboration between the eight Dutch University Medical Centres. ${ }^{14}$ In short, the Pearl Neurodegenerative Diseases focuses on the role of biomarkers in the early diagnosis, differential diagnosis, and prognosis of neurodegenerative diseases, in particular AD. ${ }^{14}$ Eligible for inclusion are individuals referred to one of the eight academic memory clinics for the evaluation of cognitive problems, with a Clinical Dementia Rating scale of 0, 0.5, or 1, and a Mini-Mental State Examination (MSSE) of 20 or higher. Harmonised protocols are used to collect clinical data and biomaterials from patients who provide written informed consent. ${ }^{14}$ Individuals are classified as having subjective cognitive impairment $(\mathrm{SCl})$, mild cognitive impairment $(\mathrm{MCl})$, or dementia, using a diagnostic procedure that is harmonised across the participating centres. ${ }^{14}$ 
For the present cross-sectional analyses, we used data from the first 258 patients who were enrolled in the database between March 2009 and December 2013 and successfully underwent lumbar puncture, which is optional for participants in the Pearl. From these, we selected those patients diagnosed with $A D$ or its potential prodromal stages (ie, amnestic $\mathrm{MCl}$ or $\mathrm{SCl})$, thereby excluding individuals with non-amnestic $\mathrm{MCl}(n=26)$ or other types of dementia ( $n=37$ ). To distinguish amnestic from non-amnestic $\mathrm{MCl}$, objective memory deficits were defined as age-, sex-, and educational level-adjusted immediate or delayed recall scores on the 15 Word-Auditory Verbal Learning Test (AVLT) $)^{15,16}$ that were at least 1.5 standard deviation below the normative mean. ${ }^{17}$ Individuals in whom the presence of objective deficits could not be verified were excluded $(n=3)$.

The Pearl Neurodegenerative Diseases was approved by the Medical Ethics Review Committee of the Free University (VU) Medical Centre Amsterdam, and written informed consent was obtained from patients enrolled in the dataset.

\section{Cerebrospinal fluid measures}

As described previously, ${ }^{14}$ lumber puncture was performed at the L3/L4 or L4/L5 intervertebral space and $3 \mathrm{ml}$ of the collected CSF was aliquoted in $0.5 \mathrm{ml}$ samples, which were stored at $-80^{\circ} \mathrm{C}$. From these samples, $A \beta$, tau, and $p$-tau were measured using Innotest ${ }^{\circledR}$ Enzyme-Linked Immunosorbent Assays (Innogenetics, Ghent, Belgium). ${ }^{14}$ These assays were performed at the department of Clinical Chemistry of the VU Medical Centre Amsterdam, a laboratory that was masked to all clinical data.

CSF levels of insulin were measured at the same laboratory in the CSF volume that remained after aliquots had been taken for $A \beta$, tau, and $p$-tau assays, a leftover volume that ranged between 75 and $150 \mu \mathrm{L}$ per patient. Insulin levels were determined by radioimmunoassay with a detection limit of $1.2 \mathrm{pmol} / \mathrm{L}$. The limited amount of CSF available prevented us from measuring insulin in duplicate. Note that patients were not required to fast prior to lumbar puncture.

\section{Cognitive performance}

As part of the diagnostic workup, patients completed a standardised battery of cognitive tests. ${ }^{14}$ We selected neuropsychological tests that reflect global cognition (ie, MMSE ${ }^{18}$ ) and memory performance (ie, AVLT ${ }^{15,16}$ ), the latter because insulin signalling has been primarily related to memory performance. ${ }^{19,20}$ Total immediate and delayed recall scores of the ALVT were combined into one composite memory score. To this end, raw test scores were transformed into z-scores and averaged. 


\section{Covariates}

A detailed description of the covariates (ie, educational level, medical history, and lifestyle factors) is provided in the Supplement.

\section{Statistical analyses}

Differences in demographic and clinical characteristics across the diagnostic groups (ie, $\mathrm{SCl}, \mathrm{aMCl}$, and $\mathrm{AD}$ ) were evaluated using analysis of variance for continuous variables and chi-square tests for categorical variables. Because there are currently limited data on variables that determine CSF levels of insulin, we used univariate linear regression to explore the association between CSF insulin and the available set of demographic and clinical variables known to be associated with cognitive performance. In these analyses, CSF insulin was taken as the dependent variable. Variables that showed a statistically significant association with CSF insulin were considered as covariates in subsequent multivariate analyses.

With use of analysis of covariance, we examined whether CSF insulin levels differed between the diagnostic groups, independent of potential confounding variables. Multiple linear regression analyses were then conducted to examine the association of CSF insulin with cognitive performance (ie, MMSE and memory score) and CSF biomarkers of AD (ie, $A \beta$, tau, and $p$-tau). In these main analyses, which were performed across and within each diagnostic group, CSF insulin was the independent variable. Two predefined models of adjustment were used (model 1: centre; model 2: model $1+$ age and sex). The third and final model also included those variables being associated with CSF insulin.

As previous studies have suggested that the effects of insulin (administration) on cognitive performance in AD may differ according to sex and APOE- $\varepsilon 4$ genotype, ${ }^{10,}{ }^{21-29}$ we explored whether any association between CSF insulin and cognitive performance or Alzheimer pathology differed by these variables. Likewise, we examined whether any association differed by the presence of diabetes given that diabetes is characterised by abnormal insulin signalling, at least in the periphery. In case of significant interaction, stratified analyses were performed.

As a sensitivity analysis, we reanalysed the data with inclusion of individuals excluded from the main analyses because of missing data. These analyses were only adjusted for participating centre. All analyses were performed with use of SPSS for Windows, version 23.0 (IBM SPSS, IBM Corp, Armonk, NY, USA). A two-sided p-value < 0.05 was considered statistically significant, except for interaction analyses where the significance level was set at 0.10 to compensate for the loss of statistical power in these analyses. ${ }^{30}$ Assumptions of linear regression were verified prior to analyses. 


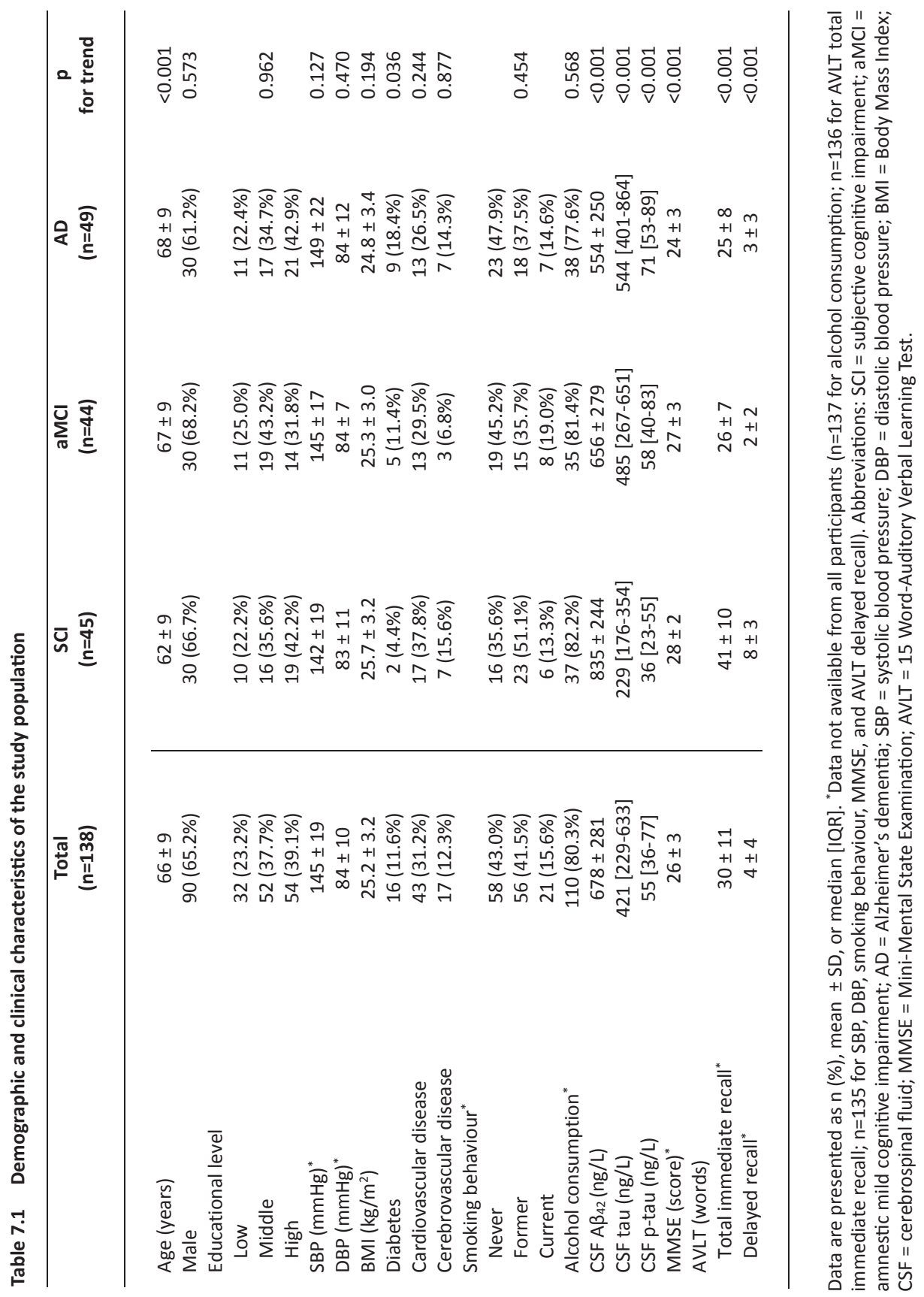




\section{Results}

CSF insulin could be determined for 160 out of the 192 patients (83.3\%) who underwent lumbar puncture and were eligible for the present study. In the other 32 patients, there was too little CSF left after A $\beta$, tau, and p-tau assays. Of the 160 patients, $12(0.8 \%)$ had no data on diabetes status $(n=8)$ and/or APOE- $\varepsilon 4$ genotype $(n=5)$, and where therefore excluded from the study sample. An additional 10 individuals were excluded because of missing data on covariates, resulting in a final study population of 138 individuals $(n=136$ for analyses with cognitive performance). Individuals who were excluded because of missing data did not differ from those who were included in the present analyses (Table S7.1, Supplement).

Table 7.1 shows the demographic and clinical characteristics of the study population as a whole and stratified by diagnostic group (ie, SCl ( $n=45)$; $\operatorname{aMCl}(n=44)$, and $A D(n=49)$ ). Overall, patients were in late adulthood (66 \pm 9 years), with a slight male predominance (65.2\%). Of note, the cardiovascular risk profile was similar across the diagnostic groups, except for the presence of diabetes, which was least common in the $\mathrm{SCl}$ group.

\section{CSF insulin}

Table 7.2 shows the univariate associations of clinical and demographic variables with CSF levels of insulin. Diabetes status and higher body mass index were associated with higher CSF insulin levels. For other variables, no statistically significant associations were observed.

\section{CSF insulin and syndromal diagnosis}

Median (interquartile range) levels of CSF insulin in individuals with $\mathrm{SCl}, \mathrm{aMCl}$, and $\mathrm{AD}$ were $3.77 \mathrm{pmol} / \mathrm{L}$ (3.34-4.39), $3.61 \mathrm{pmol} / \mathrm{L}$ (3.22-4.36), and $3.98 \mathrm{pmol} / \mathrm{L}$ (3.57-4.77), respectively. Despite the seemingly higher levels of CSF insulin in AD, CSF insulin did not differ statistically significant between the groups $(p=0.136)$, nor was the linear trend across the diagnostic statistically significant ( $p$ for trend=0.059). Sex and APOE- $\varepsilon 4$ genotype did not modify these findings ( $p$ for interaction $=0.707$ and 0.120 , respectively). When diabetes status and body mass index were included as covariates, pairwise comparisons between the diagnostic groups confirmed that CSF insulin did not differ between the groups (all $p>0.105$ ).

\section{CSF insulin and cognitive performance}

The left panel of Table 7.3 shows the association of CSF insulin with cognitive performance across the groups and within each diagnostic group. After adjustment for centre, CSF insulin was not associated with cognitive performance in the overall study population, 
Table 7.2 Univariate associations of clinical and demographic characteristics with insulin levels in the cerebrospinal fluid

\begin{tabular}{|c|c|c|c|c|}
\hline & $\begin{array}{c}n \\
\left(n_{\max }=138\right)\end{array}$ & $\beta$ & $(95 \% \mathrm{Cl})$ & p-value \\
\hline Age & 138 & 0.012 & $(-0.158 ; 0.181)$ & 0.890 \\
\hline \multicolumn{5}{|l|}{ Male } \\
\hline male vs. female & 138 & 0.214 & $(-0.139 ; 0.566)$ & 0.234 \\
\hline \multicolumn{5}{|l|}{ Educational level } \\
\hline high vs. medium & 138 & 0.068 & $(-0.317 ; 0.453)$ & 0.727 \\
\hline high vs. low & & -0.185 & $(-0.627 ; 0.258)$ & 0.410 \\
\hline SBP & 135 & 0.101 & $(-0.070 ; 0.271)$ & 0.245 \\
\hline DBP & 135 & 0.039 & $(-0.132 ; 0.211)$ & 0.650 \\
\hline $\mathrm{BMI}$ & 138 & 0.261 & $(0.097 ; 0.425)$ & 0.002 \\
\hline \multicolumn{5}{|l|}{ Diabetes } \\
\hline yes vs. no & 138 & 1.152 & $(0.662 ; 1.642)$ & $<0.001$ \\
\hline \multicolumn{5}{|l|}{ Cardiovascular disease } \\
\hline \multicolumn{5}{|c|}{ Cerebrovascular disease } \\
\hline \multicolumn{5}{|l|}{ Smoking behaviour } \\
\hline current vs. former & 135 & -0.025 & $(-0.534 ; 0.484)$ & 0.923 \\
\hline $\begin{array}{l}\text { current vs.never } \\
\text { Alcohol consumptior }\end{array}$ & & 0.096 & $(-0.410 ; 0.603)$ & 0.707 \\
\hline yes vs. no & 137 & -0.080 & $(-0.506 ; 0.347)$ & 0.713 \\
\hline
\end{tabular}

Data are presented as standardised regression coefficients ( $95 \%$ confidence intervals) for continuous variables and between-group differences ( $95 \%$ confidence intervals) for categorical variables, the latter expressed in standard deviations of cerebrospinal fluid insulin levels. Insulin levels were transformed with the natural logarithm prior to analysis. Abbreviations: SBP = systolic blood pressure; $\mathrm{DBP}=$ diastolic blood pressure; $\mathrm{BMI}$ = Body Mass Index.

nor within the groups of individuals with aMCI or $\mathrm{AD}$ (model 1). Among individuals with $\mathrm{SCl}$, higher CSF insulin levels were associated with worse memory function (model 1), but this association lost its statistical significance once adjustments were made for age and sex (model 2).

Multiplicative interaction analyses revealed that the null associations between CSF insulin and cognitive performance did not differ by the presence of diabetes (all $p$ for interaction > 0.614) or APOE- $\varepsilon 4$ genotype (all $p>0.425$ ), but did differ by sex (all $p$ $<0.059$ ). Subsequent stratified analyses (Table 7.3, right panel) showed that among women, but not among men, higher CSF insulin were associated with worse cognitive performance. These associations reached statistical significance for performance on the MMSE (model 3). 


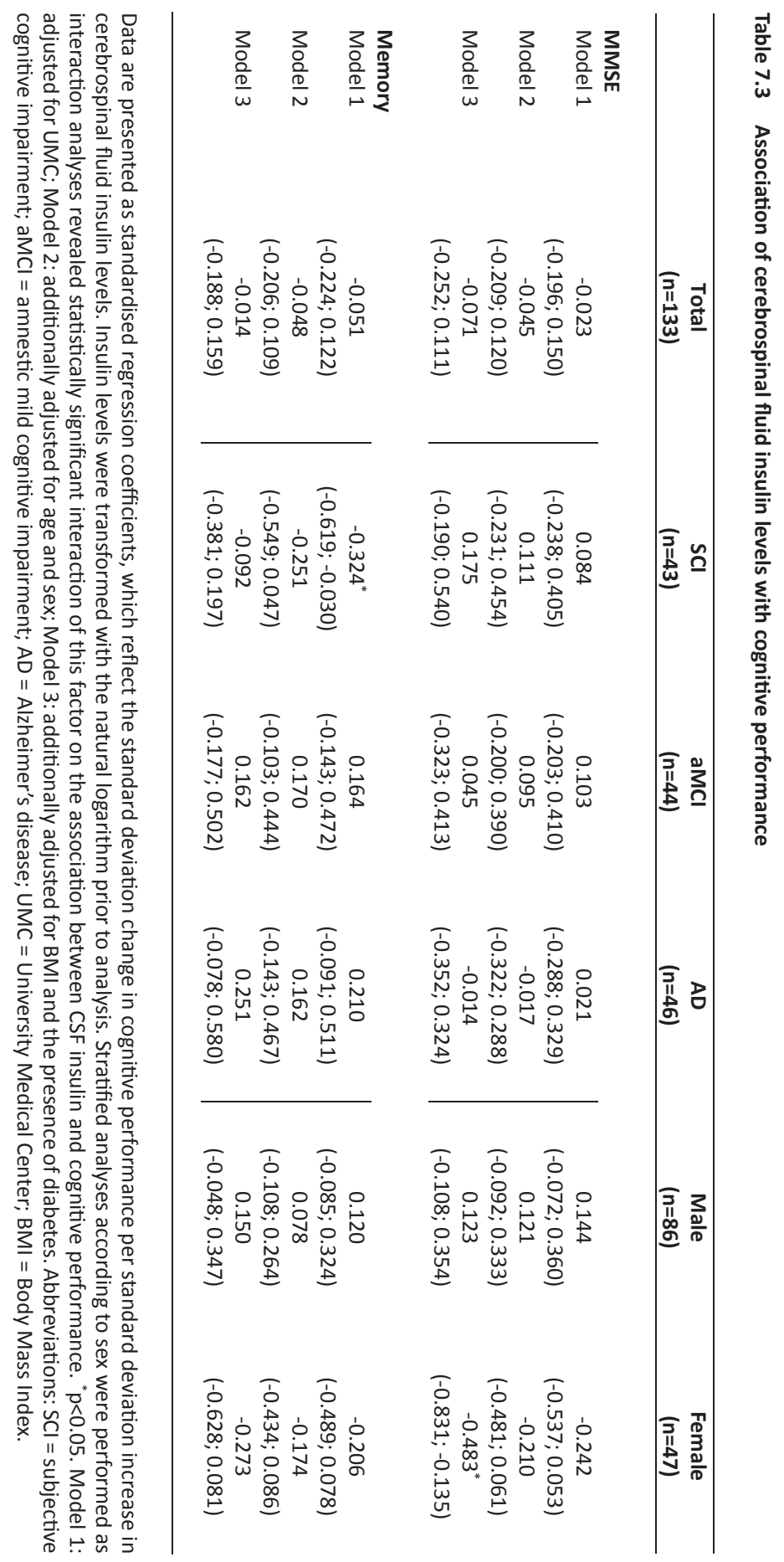




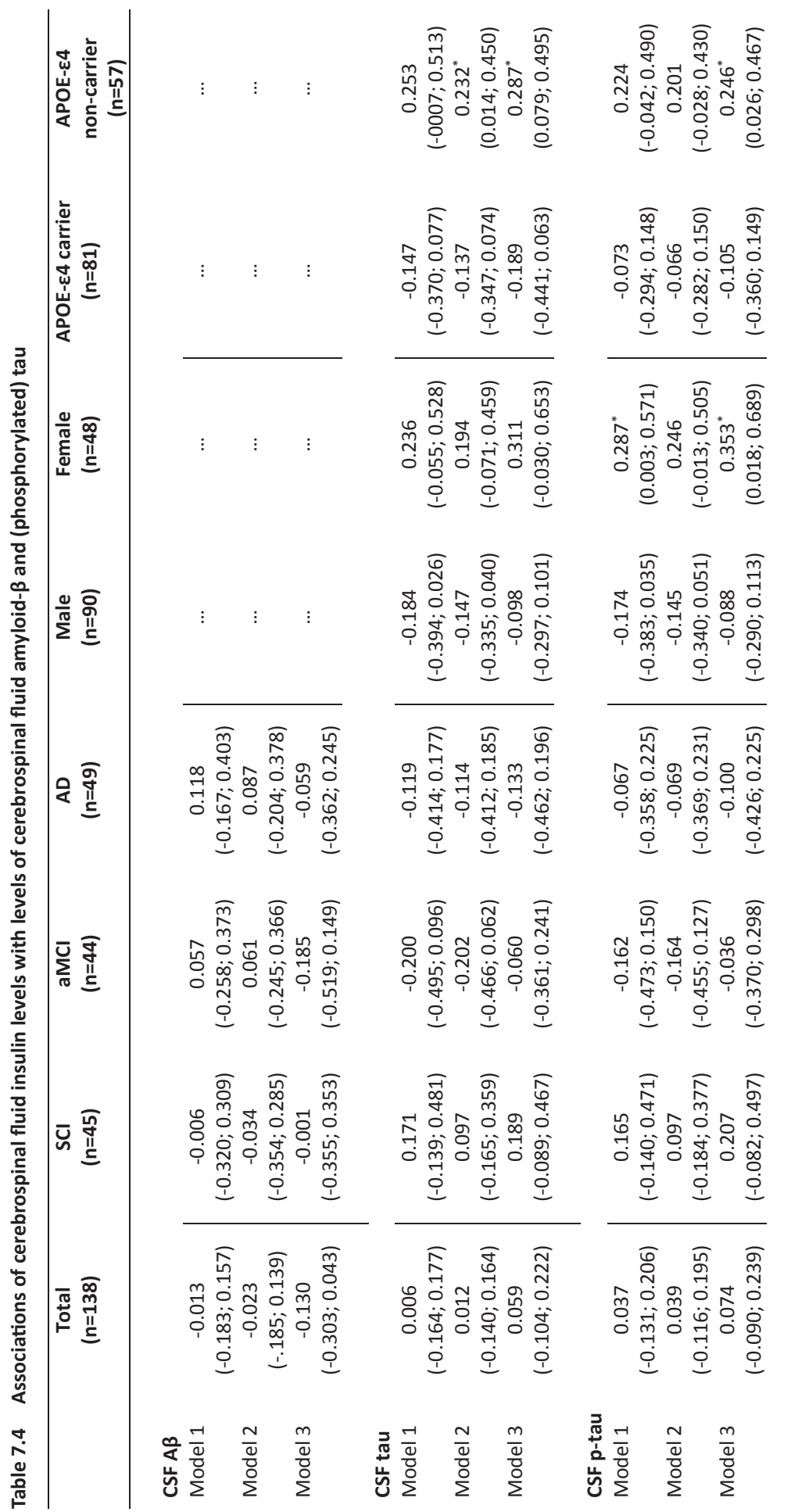

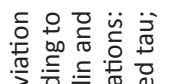

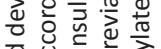
웅 㐘

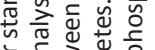

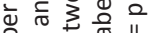

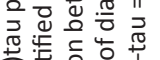
을 은

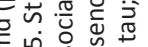

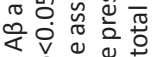

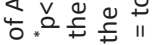

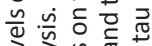

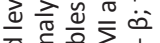
을 त

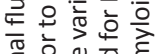

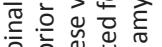

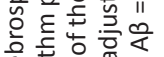
ปั

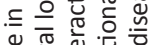

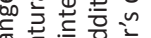
元

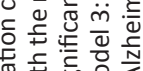

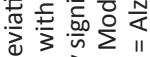

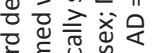

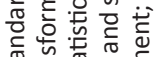

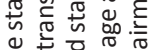

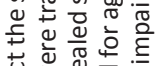
उ

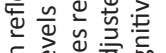
는

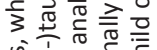
产党

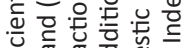

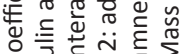

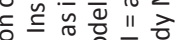

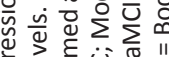

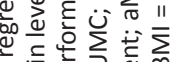

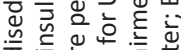

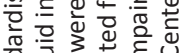

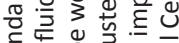

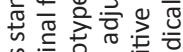

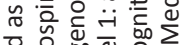

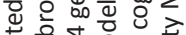

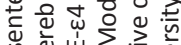
山ै.

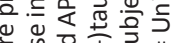

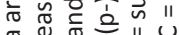

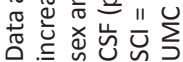




\section{CSF insulin and CSF biomarkers of AD}

The left panel of Table 7.4 shows the associations of CSF insulin with CSF biomarkers of $A D$ across and within each diagnostic group. In brief, CSF insulin was not associated with CSF $A \beta$, nor with levels of tau or $p$-tau (models 1 to 3 ). Similar null associations were observed within the individual diagnostic groups

Interaction analyses showed that diabetes did not modify these null associations (all $p$ for interaction $>0.170$ ), while sex and APOE- 4 genotype did, at least for the association between CSF insulin and ( $p$-)tau (all $p<0.017$ and $<0.096$, respectively). Subsequent stratified analyses (Table 7.4, right panel) showed that among women, but not among men, higher CSF insulin levels were statistically significantly associated with higher levels of tau and tended to be associated with higher levels of p-tau (model 3). In APOE- $\varepsilon 4$ noncarriers, but not in carriers, higher CSF insulin was statistically significantly associated with both higher tau and p-tau levels (model 3). Notably, associations were strongest among female patients who were non-carriers of the APOE- $\varepsilon 4$ allele (data not shown).

\section{Sensitivity analysis}

When the data were reanalysed with inclusion of individuals who were initially excluded because of missing data on the covariates, qualitatively similar results were obtained (Table S7.2, Supplement).

\section{Discussion}

The present study, which is the largest to date to investigate the association of CSF insulin with cognitive performance and CSF biomarkers of $A D$, has two main findings. First, CSF levels of insulin were similar across diagnostic groups of cognitive impairment (ie, $\mathrm{SCl}, \mathrm{aMCl}$, and $\mathrm{AD}$ ). Second, among women and non-carriers of the APOE- $\varepsilon 4$ allele, higher levels of CSF insulin were associated with worse cognitive performance and/or higher levels of CSF ( $p$-)tau.

Over the last decades, there has been a growing body of evidence for involvement of abnormal insulin signalling in the aetiology of AD. ${ }^{1-4}$ This has formed the basis for studies that aim to improve cognition in individuals with $A D$ or aMCl through intranasal administration of insulin. 24-28,31,32 There is a common thread emerging from this literature, in that sex and APOE- $\varepsilon 4$ genotype may modulate the cerebral effects of insulin in humans. Previous epidemiological studies have shown that peripheral hyperinsulinaemia ${ }^{23}$ and insulin resistance ${ }^{33}$ are only associated with cognitive performance ${ }^{33}$ and Alzheimer's risk $^{23}$ among women ${ }^{33}$ and non-carriers of APOE- $\varepsilon 4 .{ }^{23}$ Likewise, several studies suggest that women and those without any APOE- $\varepsilon 4$ allele seem to benefit most from intranasal 
insulin administration, ${ }^{26-28}$ although this has not been confirmed by others. ${ }^{24,25}$

The observed modifying effects of sex and APOE-ع4 genotype may also partly explain the heterogeneous findings across the handful of studies ${ }^{9-13}$ that have previously examined CSF insulin in relation to cognitive performance. Thus far, these studies have mainly focused on whether levels of CSF insulin are altered in $A D$, and reported CSF insulin to be increased, ${ }^{9}$ decreased, ${ }^{10,12}$ or unchanged ${ }^{11,13}$ in individuals with $A D$ as compared to healthy individuals ${ }^{9-11,13}$ or those with $\mathrm{SCl} .{ }^{12}$ Studies on the association between CSF insulin and actual cognitive performance have also yielded conflicting results. ${ }^{10-12}$ One other factor likely contributing to the inconsistency across studies is the use of different study populations. For example, studies reporting decreased levels of CSF insulin in $A D^{10}$, ${ }^{12}$ excluded individuals with diabetes, while the present study provides evidence that the presence of diabetes is associated with higher levels of CSF insulin. Other differences between the populations concern the severity of the dementia and the use of different reference groups.

Despite the numerous reviews that have been published on the mechanisms through which cerebral insulin might interfere with $A \beta$ and tau metabolism $\left(\mathrm{eg}^{4},{ }^{44}\right)$, only one previous study has actually evaluated CSF insulin in relation to CSF A $\beta$ and ( $p$-)tau levels. ${ }^{12}$ The overall null results of that study are in line with the null associations we observed in our overall study population. We, however, now show that higher levels of CSF insulin were associated with higher levels of CSF ( $p$-)tau in women and noncarriers of the APOE- $\varepsilon 4$ allele, which reinforces data from experimental studies linking insulin to tau metabolism. From a pathophysiological viewpoint, higher levels of CSF insulin, potentially reflecting cerebral insulin resistance and thus deficient brain insulin signalling, may result in reduced activity of the phosphoinositide 3-kinase-protein kinase B pathway, which, in turn, leads to overactivation of glycogen synthase kinase-3 and, consequently, hyperphosphorylation of tau. ${ }^{34}$ That the association between CSF insulin and tau differs with APOE- $\varepsilon 4$ genotype could be explained by a higher degree of insulin resistance among non-carriers relative to carriers of the APOE- $\varepsilon 4$ allele, as illustrated by differences in insulin-mediated glucose disposal rates. ${ }^{21}$ The sex differences observed in our study are in line with experimental data showing that the brains of male and female rats are differentially sensitive to the effects of insulin. ${ }^{35}$ Notably, we did not observe any association between CSF levels of insulin and $A \beta$. A potential explanation for this finding are the complex and dual effects insulin (signalling) may have on $A \beta$ clearance. ${ }^{4}$ On the one hand, insulin may promote $A \beta$ clearance by accelerating its intraneuronal transport and increasing protein levels of the insulin degrading enzyme (IDE), a metalloprotease that is able to degrade $A \beta .{ }^{4}$ On the other hand, IDE also catabolises insulin, for which it has a higher affinity, which means that insulin at the same time can inhibit $A \beta$ degradation. ${ }^{4}$ 
The present study had some limitations. First, CSF samples were not always taken in the fasting state, nor was lumbar puncture performed at a fixed time of the day, while insulin secretion in humans seems to follow a circadian rhythm. ${ }^{36}$ This potential variation in the measurement of CSF insulin may have affected our results. A second limitation that may have further affected the estimates of the the association between CSF insulin and CSF $p$-tau concerns the use of an antibody for $p$-tau that was specific for phosphorylation at threonine 181, while deficient insulin signalling leads to a widespread phosphorylation of tau at multiple sites. ${ }^{37}$ Nevertheless, these limitations are unlikely to explain the observed modulating effects of either sex or APOE- $\varepsilon 4$ genotype. Next, it can be questioned whether higher CSF insulin levels reflect cerebral insulin resistance the way hyperinsulinaemia in the plasma reflects peripheral insulin resistance. Note, however, that individuals with diabetes, who are the most likely to have cerebral insulin resistance despite the fact that cerebral and peripheral resistance not necessarily coincide, ${ }^{6}$ had higher levels of CSF insulin. Fourth, multiple analyses were performed without correction for multiple testing, though stratified analyses were only performed in case of statistically significant interaction with a clear a priori hypothesis. In addition, we consider it unlikely that the pattern of effect modification we observed, which aligns with previous research, is solely explained by the play of chance. Finally, no blood samples were taken at the time of lumbar puncture. This is unfortunate because such data would have provided valuable information on how peripheral and cerebral insulin metabolism may interact in relation to cognitive performance and Alzheimer's pathology.

In conclusion, CSF insulin levels did not differ between the diagnostic groups of cognitive impairment, but our results do indicate that higher CSF insulin levels are related to impairment in cognitive performance and biomarkers of $A D$ among women and noncarriers of the APOE- $\varepsilon 4$ allele. As such, our data indicate that potential involvement of cerebral insulin (resistance) in the pathogenesis of $A D$ is not uniform across individuals. Future studies are needed to determine whether endogenous high CSF insulin levels indeed reflect cerebral insulin resistance, as this might have implications for selection of patients who may benefit from the use intranasal of insulin to improve or preserve cognitive performance. Longitudinal studies are required to strengthen the pathophysiological relevance of our findings. 


\section{References}

1. Craft, S., Insulin resistance syndrome and Alzheimer disease: pathophysiologic mechanisms and therapeutic implications. Alzheimer Dis Assoc Disord, 2006. 20(4): p. 298-301.

2. Schioth, H.B., et al., Brain insulin signaling and Alzheimer's disease: current evidence and future directions. Mol Neurobiol, 2012. 46(1): p. 4-10.

3. Cholerton, B., L.D. Baker, and S. Craft, Insulin, cognition, and dementia. Eur J Pharmacol, 2013. 719(1-3): p. 170-9.

4. Chen, Y., et al., Deregulation of brain insulin signaling in Alzheimer's disease. Neurosci Bull, 2014. 30(2): p. 282-94.

5. Steen, E., et al., Impaired insulin and insulin-like growth factor expression and signaling mechanisms in Alzheimer's disease--is this type 3 diabetes? J Alzheimers Dis, 2005. 7(1): p. 63-80.

6. Banks, W.A., J.B. Owen, and M.A. Erickson, Insulin in the brain: there and back again. Pharmacol Ther, 2012. 136(1): p. 82-93.

7. Kern, W., et al., Low cerebrospinal fluid insulin levels in obese humans. Diabetologia, 2006. 49(11): p. 2790-2.

8. Plata-Salaman, C.R., Insulin in the cerebrospinal fluid. Neurosci Biobehav Rev, 1991. 15(2): p. 243-58.

9. Fujisawa, Y., K. Sasaki, and K. Akiyama, Increased insulin levels after OGTT load in peripheral blood and cerebrospinal fluid of patients with dementia of Alzheimer type. Biol Psychiatry, 1991. 30(12): p. 1219-28.

10. Craft, S., et al., Cerebrospinal fluid and plasma insulin levels in Alzheimer's disease: relationship to severity of dementia and apolipoprotein E genotype. Neurology, 1998. 50(1): p. 164-8.

11. Molina, J.A., et al., Cerebrospinal fluid levels of insulin in patients with Alzheimer's disease. Acta Neurol Scand, 2002. 106(6): p. 347-50.

12. Gil-Bea, F.J., et al., Insulin levels are decreased in the cerebrospinal fluid of women with prodomal Alzheimer's disease. J Alzheimers Dis, 2010. 22(2): p. 405-13.

13. Johansson, P., et al., Serum but not cerebrospinal fluid levels of insulin-like growth factor-I (IGF-I) and IGF-binding protein-3 (IGFBP-3) are increased in Alzheimer's disease. Psychoneuroendocrinology, 2013. 38(9): p. 1729-37.

14. Aalten, P., et al., The Dutch Parelsnoer Institute--Neurodegenerative diseases; methods, design and baseline results. BMC Neurol, 2014. 14: p. 254.

15. Brand, N. and J. Jolles, Learning and retrieval rate of words presented auditorily and visually. J Gen Psychol, 1985. 112(2): p. 201-10.

16. Rey, A., L'examen clinique and psychology. 1958: Oxford England: Presses Universitaires.

17. Van der Elst, W., et al., Rey's verbal learning test: normative data for 1855 healthy participants 24-81 years and the influence of age, sex, education, and mode of presentation. J Int Neuropsychol Soc, 2005. 11(3): p. 290-302.

18. Folstein, M.F., S.E. Folstein, and P.R. McHugh, "Mini-mental state". A practical method for grading the cognitive state of patients for the clinician. J Psychiatr Res, 1975. 12(3): p. 189-98.

19. Watson, G.S. and S. Craft, Modulation of memory by insulin and glucose: neuropsychological observations in Alzheimer's disease. Eur J Pharmacol, 2004. 490(1-3): p. 97-113.

20. Biessels, G.J. and L.P. Reagan, Hippocampal insulin resistance and cognitive dysfunction. Nat Rev Neurosci, 2015. 16(11): p. 660-71.

21. Craft, S., et al., Insulin metabolism in Alzheimer's disease differs according to apolipoprotein E genotype and gender. Neuroendocrinology, 1999. 70(2): p. 146-52.

22. Craft, S., et al., Insulin effects on glucose metabolism, memory, and plasma amyloid precursor protein in Alzheimer's disease differ according to apolipoprotein-E genotype. Ann N Y Acad Sci, 2000. 903: p. 222-8.

23. Kuusisto, J., et al., Association between features of the insulin resistance syndrome and 
Alzheimer's disease independently of apolipoprotein E4 phenotype: cross sectional population based study. BMJ, 1997. 315(7115): p. 1045-9.

24. Claxton, A., et al., Long-acting intranasal insulin detemir improves cognition for adults with mild cognitive impairment or early-stage Alzheimer's disease dementia. J Alzheimers Dis, 2015. 44(3): p. 897-906.

25. Claxton, A., et al., Sex and ApoE genotype differences in treatment response to two doses of intranasal insulin in adults with mild cognitive impairment or Alzheimer's disease. J Alzheimers Dis, 2013. 35(4): p. 789-97.

26. Benedict, C., et al., Differential sensitivity of men and women to anorexigenic and memoryimproving effects of intranasal insulin. J Clin Endocrinol Metab, 2008. 93(4): p. 1339-44.

27. Reger, M.A., et al., Effects of intranasal insulin on cognition in memory-impaired older adults: modulation by APOE genotype. Neurobiol Aging, 2006. 27(3): p. 451-8.

28. Reger, M.A., et al., Intranasal insulin administration dose-dependently modulates verbal memory and plasma amyloid-beta in memory-impaired older adults. J Alzheimers Dis, 2008. 13(3): p. 323-31.

29. Craft, S., et al., Insulin dose-response effects on memory and plasma amyloid precursor protein in Alzheimer's disease: interactions with apolipoprotein E genotype. Psychoneuroendocrinology, 2003. 28(6): p. 809-22.

30. Selvin, S., Statistical Analysis of Epidemiologic Data. 1996, New York, NY: Oxford University Press.

31. Craft, S., et al., Intranasal insulin therapy for Alzheimer disease and amnestic mild cognitive impairment: a pilot clinical trial. Arch Neurol, 2012. 69(1): p. 29-38.

32. Reger, M.A., et al., Intranasal insulin improves cognition and modulates beta-amyloid in early AD. Neurology, 2008. 70(6): p. 440-8.

33. Ekblad, L.L., et al., Insulin resistance is associated with poorer verbal fluency performance in women. Diabetologia, 2015. 58(11): p. 2545-53.

34. El Khoury, N.B., et al., Insulin dysfunction and Tau pathology. Front Cell Neurosci, 2014. 8: p. 22.

35. Clegg, D.J., et al., Differential sensitivity to central leptin and insulin in male and female rats. Diabetes, 2003. 52(3): p. 682-7.

36. Boden, G., et al., Evidence for a circadian rhythm of insulin secretion. Am J Physiol, 1996. 271(2 Pt 1): p. E246-52.

37. Clodfelder-Miller, B.J., et al., Tau is hyperphosphorylated at multiple sites in mouse brain in vivo after streptozotocin-induced insulin deficiency. Diabetes, 2006. 55(12): p. 3320-5. 


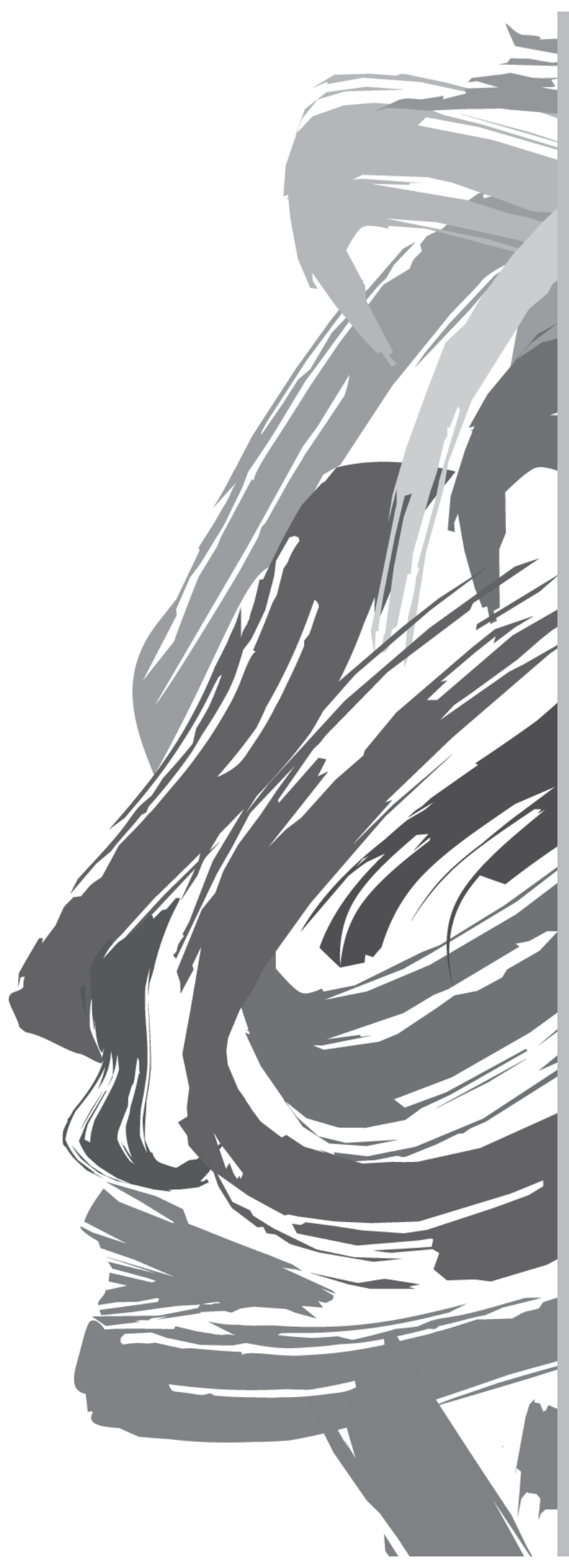


Supplement to

Chapter 7 


\section{Chapter 7}

\section{Extended methods}

\section{Covariates}

Information on educational level, medical history, and lifestyle factors was obtained in an interview with the patient and his/her caregiver. ${ }^{1}$ Eight degrees of educational level were distinguished and classified as low (ie, no education, primary education, or lower vocational education), intermediate (ie, intermediate general secondary education, intermediate vocational education, or higher general secondary education), or high (ie, higher vocational education or university).

Cardiovascular disease was defined as (a history of) hypertension, hyperlipidaemia, cardiac arrhythmias, angina pectoris, myocardial infarction, coronary artery disease, carotid artery stenosis, and/or peripheral artery disease. Cerebrovascular disease was defined as a history of transient ischaemic attack, reversible ischaemic neurologic deficit, and/or stroke. Patients were asked whether they had been diagnosed with diabetes of either type.

Current alcohol consumption was classified as none or any. Smoking behaviour was categorised into current, former, or never smoking.

Clinicians conducted a standardised psychical examination, which included a single blood pressure measurement and recording of height and weight to determine the body mass index. Genomic DNA was extracted from blood samples with the polymerase chain reaction technique and used to determine the apolipoprotein $\mathrm{E} \varepsilon 4$ (APOE-ع4) genotype. ${ }^{1}$

\section{References}

1. Aalten, P., et al., The Dutch Parelsnoer Institute--Neurodegenerative diseases; methods, design and baseline results. BMC Neurol, 2014. 14: p. 254. 
Table S7.1 Characteristics of individuals included and excluded from the present analyses

\begin{tabular}{|c|c|c|c|c|}
\hline & $\begin{array}{c}\text { Included } \\
(n=138)\end{array}$ & $\begin{array}{c}\text { Excluded } \\
(n=22)\end{array}$ & Missing* & p-value ${ }^{\dagger}$ \\
\hline Age (years) & $66 \pm 9$ & $63 \pm 11$ & 0 & 0.232 \\
\hline Male & $90(65.2 \%)$ & $17(77.3 \%)$ & 0 & 0.265 \\
\hline Educational level & & & 1 & 0.853 \\
\hline Low & $32(23.2 \%)$ & $6(27.3 \%)$ & & \\
\hline Middle & $52(37.7 \%)$ & $7(33.3 \%)$ & & \\
\hline High & $54(39.1 \%)$ & $8(38.1 \%)$ & & \\
\hline SBP (mmHg) & $145 \pm 19$ & $139 \pm 17$ & 4 & 0.163 \\
\hline $\mathrm{DBP}(\mathrm{mmHg})$ & $84 \pm 10$ & $79 \pm 9$ & 4 & 0.065 \\
\hline BMI $\left(\mathrm{kg} / \mathrm{m}^{2}\right)$ & $25.2 \pm 3.2$ & $25.7 \pm 1.6$ & 13 & 0.684 \\
\hline Diabetes & $16(11.6 \%)$ & $1(7.1 \%)$ & 8 & 0.615 \\
\hline Cardiovascular disease & $43(31.2 \%)$ & $5(27.8 \%)$ & 4 & 0.770 \\
\hline Cerebrovascular disease & $17(12.3 \%)$ & $4(22.2 \%)$ & 4 & 0.247 \\
\hline Smoking behaviour & & & 2 & 0.549 \\
\hline Never & $58(43.0 \%)$ & $7(35.0 \%)$ & & \\
\hline Former & $56(41.5 \%)$ & $8(40.0 \%)$ & & \\
\hline Current & $21(15.6 \%)$ & $4(25.0 \%)$ & & \\
\hline Alcohol consumption & $110(80.3 \%)$ & $14(70.0 \%)$ & 2 & 0.291 \\
\hline CSF insulin (pmol/L) & & $38[3.4-4.8]$ & 0 & 0.194 \\
\hline $\operatorname{CSF} A \beta_{42}(\mathrm{ng} / \mathrm{L})$ & $678 \pm 281$ & $753 \pm 313$ & 0 & 0.254 \\
\hline CSF tau (ng/L) & 421 [229-633] & 336 [187-492] & 0 & 0.215 \\
\hline CSF p-tau (ng/L) & $55[36-77]$ & $44[28-73]$ & 0 & 0.281 \\
\hline MMSE (score) & $26 \pm 3$ & $27 \pm 3$ & 0 & 0.164 \\
\hline AVLT (words) & & & 0 & \\
\hline Total immediate recall & $30 \pm 11$ & $29 \pm 11$ & & 0.575 \\
\hline Delayed recall & $4 \pm 4$ & $4 \pm 4$ & & 0.884 \\
\hline
\end{tabular}

Data are presented as $n(\%)$, mean $\pm S D$, or median [IQR]. *Number of missings for a specific variable. ${ }^{\dagger} \mathrm{P}$-value for difference between individuals being included and excluded from the present analyses as evaluated with use of independent samples t-tests for continuous variables and Chi-square tests for categorical variables. Abbreviations: $\mathrm{SBP}=$ systolic blood pressure; $\mathrm{DBP}=$ diastolic blood pressure; $\mathrm{BMI}=$ Body Mass Index; $\mathrm{CSF}=$ cerebrospinal fluid; MMSE = Mini-Mental State Examination; AVLT = 15 Word-Auditory Verbal Learning Test. 
Table S7.2 Association of cerebrospinal fluid insulin levels with cognitive performance

\begin{tabular}{|c|c|c|c|c|}
\hline & Total & $\mathrm{SCl}$ & $\mathrm{aMCl}$ & $A D$ \\
\hline \multicolumn{5}{|l|}{ MMSE } \\
\hline Model 1 & $\begin{array}{c}-0.052 \\
(-0.211 ; 0.106) \\
n=157\end{array}$ & $\begin{array}{c}0.135 \\
(-0.151 ; 0.421) \\
n=52\end{array}$ & $\begin{array}{c}0.031 \\
(-0.263 ; 0.325) \\
n=49\end{array}$ & $\begin{array}{c}0.003 \\
(-0.276 ; 0.283) \\
n=56\end{array}$ \\
\hline \multicolumn{5}{|l|}{ Memory } \\
\hline Model 1 & $\begin{array}{c}-0.057 \\
(-0.215 ; 0.102) \\
n=157\end{array}$ & $\begin{array}{c}-0.179 \\
(-0.458 ; 0.100) \\
n=53\end{array}$ & $\begin{array}{c}0.079 \\
(-0.219 ; 0.377) \\
n=49\end{array}$ & $\begin{array}{c}0.137 \\
(-0.143 ; 0.418) \\
n=55\end{array}$ \\
\hline \multicolumn{5}{|l|}{$\operatorname{CSF} A \beta$} \\
\hline Model 1 & $\begin{array}{c}0.004 \\
(-0.153 ; 0.161) \\
n=160\end{array}$ & $\begin{array}{c}0.038 \\
(-0.243 ; 0.320) \\
n=54\end{array}$ & $\begin{array}{c}0.035 \\
(-0.261 ; 0.330) \\
n=49\end{array}$ & $\begin{array}{c}0.164 \\
(-0.105 ; 0.432) \\
n=57\end{array}$ \\
\hline \multicolumn{5}{|l|}{ CSF tau } \\
\hline Model 1 & $\begin{array}{c}0.023 \\
(-0.135 ; 0.180) \\
n=160\end{array}$ & $\begin{array}{c}0.103 \\
(-0.178 ; 0.384) \\
n=54\end{array}$ & $\begin{array}{c}-0.235 \\
(-0.511 ; 0.041) \\
n=49\end{array}$ & $\begin{array}{c}0.023 \\
(-0.254 ; 0.300) \\
n=57\end{array}$ \\
\hline \multicolumn{5}{|l|}{ CSF p-tau } \\
\hline Model 1 & $\begin{array}{c}0.058 \\
(-0.099 ; 0.214) \\
n=160\end{array}$ & $\begin{array}{c}0.096 \\
(-0.179 ; 0.371) \\
n=54\end{array}$ & $\begin{array}{c}-0.223 \\
(-0.515 ; 0.069) \\
n=49\end{array}$ & $\begin{array}{c}0.103 \\
(-0.173 ; 0.379) \\
n=57\end{array}$ \\
\hline
\end{tabular}

Data are presented as standardised regression coefficients, which reflect the standard deviation change in cognitive performance or CSF biomarkers of AD per standard deviation increase in cerebrospinal fluid insulin levels. Insulin levels were transformed with the natural logarithm prior to analysis. Model 1: adjusted for UMC. Abbreviations: $\mathrm{SCl}=$ subjective cognitive impairment; $\mathrm{aMCl}=$ amnestic mild cognitive impairment; $\mathrm{AD}=$ Alzheimer's disease; UMC = University Medical Centre; BMI = Body Mass Index. 
CSF insulin and Alzheimer's disease 


\section{Chapter 8}

SUMMARY AND GENERAL DISCUSSION 


\section{Summary and general discussion}

Over the past decades, it has become increasingly evident that type 2 diabetes is associated with a variety of cognitive problems, ${ }^{1}$ ranging from subtle cognitive decrements that may lead to complaints of forgetfulness and concentration loss, ${ }^{2}$ to frank dementia that clearly has a tremendous impact on patients and their families. The societal burden of diabetesassociated cognitive problems is expected to rise exponentially in the near future, not only because of the ageing of the population ${ }^{3,4}$ and the increasing life expectancy of individuals with type 2 diabetes, ${ }^{5}$ but also because of the ongoing diabetes epidemic. ${ }^{6}$, ${ }^{7}$ Against this background, the aim of the present thesis was twofold: to elucidate (1) whether the effect of type 2 diabetes on cognitive performance acts through abnormal glucose metabolism and vascular function and (2) whether abnormalities in glucose metabolism affect cognitive performance within individuals with type 2 diabetes. Put in a broader context, the present thesis tried to extend our insight into how glucose metabolism and vascular function affect our cognitive abilities.

\section{Glucose metabolism}

Given that diabetes is characterised by hyperglycaemia and insulin resistance, ${ }^{8}$ and given the multiple pathways through which these abnormalities may affect brain function, ${ }^{9-13}$ one might easily assume that hyperglycaemia and insulin resistance are involved in diabetes-associated cognitive problems. We tested this seemingly obvious, though never confirmed, hypothesis and showed that differences in processing speed and executive function and attention between individuals with type 2 diabetes and those with normal glucose metabolism were indeed largely mediated by hyperglycaemia (Chapter 2). Further, indirect evidence for a mediating role of hyperglycaemia comes from our observation in Chapter $\mathbf{2}$ that cognitive performance tended to worsen with deteriorating glucose metabolism. Hence, our data suggest that hyperglycaemia plays an important role in the onset of diabetes-associated cognitive problems, as is the case in other diabetic complications, such as nephropathy ${ }^{14}$ and retinopathy..$^{15}$ Insulin resistance, in contrast, as measured by the homeostasis model assessment (HOMAIR), ${ }^{16}$ did not explain any differences in cognitive performance between individuals with different glucose metabolism status.

Next, we asked ourselves whether abnormalities in glucose metabolism also contribute to the variance in cognitive performance seen among individuals with type 2 diabetes. Evidently, not everyone with diabetes experiences cognitive problems. ${ }^{17}$ Although numerous studies addressed the role of hyperglycaemia and insulin resistance in cognitive performance among individuals with type 2 diabetes, the magnitude of their effects remained a matter of debate. We therefore conducted a systematic review of 
the existing literature. The results of that review showed that hyperglycaemia and glucose variability were negatively, but weakly, associated with cognitive performance in people with type 2 diabetes (Chapter 5). In fact, glycated haemoglobin, the variable that was most consistently associated with cognitive performance, generally accounted for no more than $10 \%$ of the variance in cognition. Our review also highlighted the need for further studies on the role of insulin in cognition because the evidence available was insufficient to draw any conclusions. Detailed data from 806 individuals with type 2 diabetes participating in The Maastricht Study provided us a unique opportunity to further explore this issue by linking insulin-related variables to cognitive performance. In Chapter 6 it is shown that none of the insulin-related variables studied (ie, fasting insulin, C-peptide, and HOMA-IR) explains the variance in cognitive performance beyond what was already explained by demographic characteristics. Collectively, Chapters $\mathbf{5}$ and $\mathbf{6}$ provide evidence that the level of hyperglycaemia, but not of insulin resistance, explains, to some extent, why the severity of cognitive problems differs among individuals with type 2 diabetes.

Should we then conclude that insulin resistance has no effect on cognition function, regardless of the fact that experimental studies increasingly recognise the effects of insulin on the brain ${ }^{13}$ Probably not. A growing body of evidence suggests that abnormal insulin signalling in the brain is involved in Alzheimer's disease. ${ }^{13,18-20}$ Insulin signalling can, however, not be measured in the living human brain. Frequently, blood insulin concentrations are used instead, as a proxy, but these might not accurately reflect cerebral insulin levels, particularly in the presence of diabetes, as the receptor-mediated transport of insulin across the blood-brain barrier decreases with increasing insulin resistance. ${ }^{21}$ Insulin levels within the cerebrospinal fluid that surrounds the brain and spinal cord more accurately reflects levels within the central nervous system, and may therefore be more useful to assess the cerebral effects of insulin. Chapter 7 shows that in a population of patients referred to academic memory clinics, higher cerebrospinal fluid insulin levels were associated with worse cognitive performance and cerebrospinal fluid biomarkers of Alzheimer's disease, at least among women and non-carriers of the apolipoprotein E $\varepsilon 4$ allele, a genetic risk factor for Alzheimer's disease. Interestingly, in this regard, Chapter $\mathbf{7}$ also shows that individuals with diabetes had statistically significantly higher cerebrospinal fluid insulin levels than those without diabetes.

\section{Vascular function}

With diabetes typically developing in the context of vascular comorbidities, ${ }^{22,} 23$ and brain autopsy studies showing accentuated vascular damage in the brains of individuals with type 2 diabetes, ${ }^{24}$ it is generally believed that vascular function and abnormalities also play a role in diabetes-associated cognitive problems. Previous epidemiological 
studies have, however, shown conflicting results as to whether the effects of diabetes on cognitive performance are dependent of traditional vascular risk factors. ${ }^{25-30}$ In addition, reports that include mediation analyses are scarce, ${ }^{31}$ which highlights the need for further research in this area.

Hypertension, present in up to $75 \%$ of the individuals with type 2 diabetes, ${ }^{22}$ is the vascular risk factor most commonly associated with cognitive impairment ${ }^{32}$ and dementia. ${ }^{33}$ At the same time, variation in blood pressure or blood pressure variability is increasingly recognised to be associated with worse cognitive performance. ${ }^{34}$ In Chapter 2, we therefore examined the mediating effects of blood pressure-related variables and showed that these explained about one-fifth of the differences in processing speed performance between individuals with type 2 diabetes and those with normal glucose metabolism. Detailed analysis revealed that these mediating effects were mainly attributable to the use of antihypertensive medication, and thus likely due to previous exposure to elevated blood pressure. Chapter 2 also provides insight into the role of other traditional vascular risk factors in diabetes-associated differences in cognitive performance. Their effect proved to be weak, with waist circumference explaining some differences in memory function and the presence of cardiovascular disease explaining some differences in information processing speed.

Besides traditional vascular risk factors, emerging evidence suggests that vascular function is altered in type 2 diabetes $^{35-38}$ and may impact cognitive performance..$^{39-41}$ Hence, we explored the possibility of arterial stiffness and remodelling mediating diabetes-associated cognitive problems. In Chapter $\mathbf{3}$ we showed that local stiffness of the carotid artery is associated with cognitive performance, but does not account for the differences in cognitive performance between individuals with and without type 2 diabetes. Greater circumferential wall stress at the level of the carotid artery, as outlined in Chapter 4, was not associated with cognitive performance, which makes it highly unlikely that maladaptive remodelling of the carotid artery explains why individuals with type 2 diabetes generally perform worse on cognitive tasks than their counterparts without diabetes. Collectively, Chapters $\mathbf{2}$ to $\mathbf{4}$ indicate that vascular function and abnormalities are only marginally involved in the onset of diabetes-associated cognitive problems, which also explains the inconclusive findings of previous studies..$^{25-31}$

Well, then, what about the role of vascular function and abnormalities in explaining the variance in cognitive performance among individuals with type 2 diabetes? According to a recent review, ${ }^{42}$ this issue remains a matter of debate. Based on the data presented in the present thesis, their role also seems to be rather limited. More specifically, Chapter 6 provides indirect evidence that traditional vascular risk factors (ie, waist 
circumference, lipids, blood pressure, and smoking behaviour) collectively explained no more than $2.5 \%$ of the variance in cognition beyond what was already explained by demographic characteristics. In addition, the observed association between carotid stiffness and cognitive performance in people with diabetes (Chapter $\mathbf{3}$ ) was relatively weak, and therefore also unlikely to substantially contribute to the variance in cognitive performance seen in individuals with type 2 diabetes.

\section{The crossroads between glucose metabolism and vascular function}

Altogether, the results of the present thesis indicate that both metabolic and vascular factors contribute to differences in cognitive performance between individuals with and without type 2 diabetes, but do not necessarily explain the variance in cognitive performance among individuals with type 2 diabetes. In addition, some of our findings suggest that abnormalities in glucose metabolism and vascular function may interact in their effects on cognitive performance. Time to put the pieces together and resolve some apparent discrepancies in our findings.

Most importantly, we identified hyperglycaemia as the primary mediator of diabetesassociated differences in cognitive performance (Chapter 2), but at the same time showed that the degree of hyperglycaemia had little effect on the severity of diabetesassociated cognitive problems (Chapters $\mathbf{5}$ and $\mathbf{6}$ ). At first sight, these findings seem to contradict each other, but similar contradictory results have been observed for other glucose-mediated complications of type 2 diabetes. Indeed, glycaemic control has been shown to be more closely related to the development than to the progression of nephropathy ${ }^{43}$ and retinopathy. ${ }^{44}$ Several explanations may account for this discrepancy. First, it is possible that hyperglycaemia sets the stage for other mechanisms to exert their detrimental effects. Another explanation is the concept of glycaemic or metabolic memory, ${ }^{45,46}$ which refers to the sustained effects of prior glycaemic control on the development and progression of diabetic complications. Actual glucose levels may thus be less important. A third, but highly speculative, explanation is that the brain tries to protect itself from hyperglycaemia ${ }^{47}$ by reducing the transport of glucose across the blood-brain barrier. ${ }^{48,49}$ In the early stages of the disease, when diabetes is developing, this may lead to subtle hypoglycaemia in the brain, ${ }^{47}$ contributing to cognitive deficits, whereas in later stages, this protective mechanisms might be more effective in preventing the deleterious effects of overt hyperglycaemia. Alternative explanations also need to be considered. Diabetes-associated cognitive changes are known to be relatively subtle, develop early in the course of the disease, and progress only slowly over time. ${ }^{2}$ Hence, the variance in cognitive performance among individuals with type 2 diabetes might be too small to detect any correlation with the degree of hyperglycaemia. Likewise, there is a possibility of residual confounding, in that hyperglycaemia could be a proxy for an 
unknown or unmeasured confounder that is truly responsible for the differences in cognitive performance between individuals with and without type 2 diabetes.

Compared to hyperglycaemia, vascular function and abnormalities appeared less important in explaining differences in cognitive performance between individuals with and without type 2 diabetes (Chapter 2), but were of equal, albeit still limited, importance in explaining the variance in cognitive performance among individuals with type 2 diabetes (Chapters 3 and 6). Combined, this implies that vascular contributions to diabetes-associated decrements in cognitive performance are less pronounced than commonly thought, which seems to contrast with the increased burden of vascular brain damage in diabetes as seen on $\mathrm{MRI}^{50}$ and autopsy. ${ }^{24}$ It is, however, important to realise that vascular brain damage may have a metabolic origin. Recent research has suggested that rarefaction of the cerebral microvasculature is more dependent on glycaemic control than on the prevention of hypertension, ${ }^{51}$ likely because hyperglycaemia-induced oxidative stress ${ }^{9}$ lowers the bioavailability of nitric oxide. ${ }^{51}$ Chronic hyperglycaemia has also been related to blood-brain barrier dysfunction ${ }^{52}$ and reduced cerebral blood flow, ${ }^{53}$ both of which may precipitate cerebral small vessel disease. In addition, the earlier detection and more intensive treatment of vascular risk factors among individuals prone to or already diagnosed with diabetes might limit the potential detrimental effects these factors can have on the brain. Conversely, however, the intensive treatment of vascular risk factors among individuals with type 2 diabetes may also have limited our ability to accurately assess the role of these factors in diabetes-associated cognitive problems. Hence, we cannot undoubtedly conclude from our findings that the role of vascular function and abnormalities in diabetes-associated cognitive decrements is rather limited. In addition, previous studies have suggested that vascular risk factors in midlife are more closely associated with late-life than with midlife cognitive performance, ${ }^{54}$ while the present thesis focused on midlife cognitive performance.

Interestingly, we found evidence for interaction between hyperglycaemia or diabetes and hypertension in their association with cognitive performance (Chapters $\mathbf{2}$ and $\mathbf{3}$ ), which confirms the findings of some previous studies ${ }^{55-57}$ showing that the presence of the one exacerbates the negative effects of the other. A likely explanation for this interaction is the concept of cognitive reserve, which reflects the brain's ability to withstand pathology before performance is affected..$^{58}$ In this view, it is possible that the negative effects of hyperglycaemia and hypertension on the brain are strictly independent of one another, but that both contribute to accumulating brain damage that lowers the threshold for additional damage to cause objective impairments in cognitive performance. Some interplay is, however, to be expected, as it has been, for example, been suggested that hyperglycaemia increases endothelial vulnerability to elevated blood pressure. ${ }^{59}$ 
Both the current literature (Chapter 5) and our data (Chapters 2 and 6) show that insulin resistance, as measured by the HOMA-IR, is unrelated to cognitive performance in the context of diabetes. That is, it does not seem to mediate diabetes-associated differences in cognitive performance (Chapter 2), nor does it explain any variance in cognitive performance among individuals with type 2 diabetes (Chapters 5 and 6). This may indicate that insulin resistance is irrelevant in diabetes-associated cognitive problems, but could also reflect the brain's ability to protect itself from hyperinsulinaemia. Indeed, the transport of insulin across the blood-brain barrier has been shown to decrease with increasing insulin resistance. ${ }^{21} \mathrm{We}$, nonetheless, showed that individuals with selfreported diabetes had higher cerebrospinal insulin levels than their counterparts without diabetes (Chapter 7) and found that higher insulin levels within the cerebrospinal fluid were associated with worse cognitive performance and biomarkers of Alzheimer's disease, at least among women and non-carriers of the apolipoprotein E $\varepsilon 4$ allele (Chapter 7). Remarkably, type 2 diabetes was previously shown to particularly increase the risk of dementia in carriers of the apolipoprotein E $\varepsilon 4$ allele. ${ }^{25}$ In addition, the diabetesassociated increased risk of developing nonvascular dementia is comparable among men and women. ${ }^{60}$ This even puts into question whether cerebral insulin resistance is likely to be involved in diabetes-associated cognitive problems, which is further challenged by the notion that cerebral and peripheral insulin resistance not necessarily coincide ${ }^{61}$ and may have a different nature. ${ }^{62}$

In sum, based on the findings of this thesis, we believe that diabetes-associated cognitive problems are mainly attributable to hyperglycaemia, and thus can be considered as a glucose-mediated complication of type 2 diabetes. The effects of long-term exposure to elevated blood pressure should, however, not be overlooked as they may also, to a lesser extent, contribute to the cognitive sequelae of diabetes, and could exacerbate the negative cognitive effects of hyperglycaemia. Peripheral insulin resistance seems to be of no importance. Importantly, none of the variables evaluated in the present thesis explained a substantial proportion of the variance in cognitive performance among individuals with diabetes. Hence, factors other than abnormalities glucose metabolism and vascular function are likely to explain why only a subset of individuals with type 2 diabetes experience cognitive problems.

\section{Methodological considerations and challenges}

\section{Study design}

Although the present thesis enhances our understanding of diabetes-associated cognitive problems, we should not lose sight that most of the data were derived from cross-sectional observational studies. Cross-sectional studies do not allow conclusions 
regarding the temporality of associations, which introduces, among others, the possibility of reverse causality. Cognitive abilities or general intelligence at young adult age might be associated with the risk of type 2 diabetes later in life. ${ }^{63}$ Similarly, although mediation analyses in cross-sectional studies can provide useful clues, mediation analyses with longitudinal data are preferred to answer aetiological research questions. ${ }^{64,65}$ Due to the cross-sectional nature of the studies, the effects of glucose metabolism and vascular function on the progression of diabetes-associated cognitive problems or late-life cognitive performance among individuals with type 2 diabetes can only be speculated. Despite the limitations of our cross-sectional approach, we believe it is an appropriate first step in unravelling the complexity of diabetes-associated cognitive problems. In addition, due to the extensive phenotyping of The Maastricht Study, and, to a lesser extent, that of the Pearl Neurodegenerative Diseases, we were able to account for important potential confounders. We can, however, not completely exclude the possibility of residual confounding, nor can we be sure that our findings have not been influenced by overadjustment, ${ }^{66}$ for example, by adjusting for an intermediate factor that we considered to be a true confounder.

\section{Selection of the study population}

Some type of selection is inevitable in population-based cohort studies such as The Maastricht Study. The observation that participants of The Maastricht Study population were generally well educated and had a relatively well-controlled metabolic and vascular risk profile likely reflect this self-selection, which resulted in a population with a relatively high level of cognitive reserve. This may have limited our ability to accurately quantify the effects of glucose metabolism and vascular function on (a wide spectrum of) cognitive performance. In addition, it has been suggested that the brain becomes most susceptible to cognitive decline after the age of 65 when accumulating brain damage and neurodegeneration starts to exceed the reserve capacity of the brain, ${ }^{67}$ while the mean age of participants of The Maastricht Study was about 60 years. Note, however, that diabetes-associated decrements in cognitive performance are already observed in the early stages of the disease, ${ }^{2,47}$ which highlights the necessity to investigate middle-aged people to explore the nature of these problems. We, nonetheless, acknowledge that selection mechanisms may have affected the generalisability of the findings presented in this thesis.

\section{Measurement of variables}

In the present thesis, cognitive performance was used as the primary outcome. In neuropsychology, extensive neuropsychological testing is considered the gold standard to assess cognitive function. ${ }^{68}$ Such extensive testing is, however, not feasible for most epidemiological studies. Therefore, frequently, a selected number of neuropsychological 
tests are conducted to assess performance across a range of cognitive domains. A problem that then arises is the selection of tests: numerous validated tests are available and there is no consensus on which tests should be preferably used. ${ }^{68}$ Some general recommendations have, however, been made to construct an optimal test battery for the assessment of diabetes-associated cognitive decline. ${ }^{68}$ First, given that diabetesassociated decrements in cognitive performance are usually small, neuropsychological tests should be very sensitive to detect subtle differences in cognitive performance. Furthermore, tests should cover the cognitive domains frequently reported to be affected in diabetes. These include memory, information processing speed, executive function and attention. ${ }^{1,69-71}$ The concise neuropsychological test battery used in The Maastricht Study follows these recommendations, and was applied by trained personnel. So far, no need to consider the possibility of measurement errors. However, some might argue that the use of a priori chosen individual tests is preferred above the use of cognitive domain scores, as the classification of tests into domains is rather subjective and many individual tests provide information on multiple domains. Apart from the fact that there are some guidelines for the classification of tests into domains, ${ }^{72}$ the use of individual tests suffers from the same lack of consensus and thus subjectivity with regard to which test should be chosen. In addition, the use of individual tests complicates the conceptual interpretation of findings. An important issue that remains is the fact that The Maastricht Study population includes individuals with relatively preserved cognitive abilities, while diabetes-associated cognitive problems range from subtle cognitive decrements to frank dementia. It is increasingly accepted that these not necessarily form a continuum and may have a different pathophysiology, ${ }^{2}$ which implies that our results not necessarily apply to the diabetes-associated increased dementia risk.

Type 2 diabetes is characterised by insulin resistance, ${ }^{8}$ but assessing the degree of insulin resistance in individuals with diabetes can be challenging. The hyperinsulinaemiceuglycaemic clamp ${ }^{73}$ is the gold standard for insulin resistance, but its time-consuming and labour-intensive character makes it impractical for large population-based studies. Observational studies, therefore, often use surrogate markers instead. HOMA-IR is the most frequently used alternative, which is based on fasting insulin and glucose blood levels. ${ }^{16}$ Given a fixed glucose level, HOMA-IR will be higher with higher insulin concentrations. From a (patho)physiological point of view, the usefulness of insulin levels to estimate insulin resistance among individuals with type 2 diabetes can be questioned. In the early stages of the disease, pancreatic $\beta$-cells increase their insulin secretion to compensate for the increasing degree of insulin resistance. ${ }^{74}$ However, $\beta$-cells often lose their secretory capacity as the disease progresses, ${ }^{74}$ which is not accounted for in HOMA-IR, apart from the fact that glucose levels are then also likely to rise. In addition, oral glucose-lowering medication can enhance insulin secretion, ${ }^{75}$ potentially further 
obscuring HOMA-IR results. Yet, HOMA-IR has been validated in diabetes populations ${ }^{16}$ and there is currently no superior alternative.

\section{Recommendations}

\section{Clinical implications}

Over the past two decades, clinicians have become increasingly aware of the effects diabetes may have on the brain. There is also an increasing awareness among people with type 2 diabetes that their disease puts them at increased risk for cognitive problems. It was, however, not until earlier this year that the American Diabetes Association officially acknowledged cognitive dysfunction as a complication of type 2 diabetes in their standards of medical care. ${ }^{76}$ Recognising the problem is a first step, but it also brings up a series of new questions: what causes diabetes-associated cognitive problems?; (how) can we prevent diabetes-associated cognitive problems?; and (how) can we treat diabetes-associated cognitive problems? The work in this thesis is an effort to address some of these questions. Despite the methodological considerations outlined above, we believe our work provides some further insight into the aetiology of diabetes-associated problems and the factors that determine their severity. As such, the thesis may provide potential targets for the prevention or amelioration of cognitive problems in diabetes.

The present thesis shows that diabetes-associated differences in cognitive performance are largely explained by hyperglycaemia, and, to a lesser extent, by long-term exposure to hypertension. This makes us believe that early glycaemic and blood pressure control are promising targets for the primary prevention of diabetes-associated cognitive problems. At present, individuals with prediabetes are encouraged to change their lifestyle in order to lose weight and hopefully return to normal glucose metabolism. ${ }^{76,77}$ Guidelines also recommend the identification, and if appropriate, treatment of other vascular risk factors, including hypertension. ${ }^{76}$ There, are, however no strict targets for glycaemic control and individuals with prediabetes are seldom prescribed glucose-lowering medication to prevent deterioration in glycaemic control. ${ }^{76,77}$ Our work indicates that intensified glycaemic and blood pressure control in those without diabetes may be necessary to prevent diabetes-associated cognitive problems, contributing to the concept that this complication develops early in the course of diabetes. ${ }^{2,47}$ This adds fuel to the debate as to whether individuals with prediabetes should be treated with glucose-lowering treatment. Previous research has shown that treatment with metformin or acarbose in prediabetes decreases the rate of conversion to diabetes by 25 to $30 \%,{ }^{77,78}$ and may also have beneficial effects on the risk of cardiovascular disease. ${ }^{77}$ Glycaemic control in prediabetes has indeed previously been linked to an increased risk of cardiovascular disease. ${ }^{79}$ The prevention of cognitive problems may be just another reason to consider 
targets for glycaemic control in people without diabetes. This view is supported by results of the Outcome Reduction with Initial Glargine Intervention (ORIGIN) trial, which found that insulin therapy in prediabetes to normalise blood glucose levels tends to reduce the decline in some aspects of cognitive performance. ${ }^{80}$

Identification of factors that contribute to the variance in cognitive performance among individuals with type 2 diabetes is important for the secondary prevention of diabetesassociated cognitive problems, that is, amelioration of cognitive problems among individuals with type 2 diabetes. The present thesis shows that hyperglycaemia, carotid arterial stiffness, and traditional vascular risk factors all explain only marginal amounts of variance in cognitive performance in diabetes, which may explain why randomised controlled trials on intensified glucose-lowering, ${ }^{81-83}$ blood pressure-lowering, ${ }^{84}$ or lipidlowering ${ }^{84}$ treatment in people with type 2 diabetes have failed to show improvement in cognitive performance. This does, however, not foreclose the possibility that improving the metabolic and vascular risk profile of individuals with diabetes may have some beneficial effects. Indeed, positive effects on cognitive performance were observed in both treatment groups in most randomised trials on intensified glucose-lowering treatment, ${ }^{81,82}$ suggesting that improving glycaemic control does have beneficial effects on brain function.

\section{Future directions}

The present thesis should be considered as a first step in unravelling the complexity of diabetes-associated cognitive problems. Further research is warranted to further understand these problems. Future studies should preferably ...

... have a longitudinal design

Most of the evidence provided in this thesis comes from a series of cross-sectional analyses that need confirmation in longitudinal studies. Longitudinal studies are also needed to assess the role of glucose metabolism and vascular function in diabetesassociated (accelerated) cognitive decline, and explore the effects of midlife exposure to abnormalities in glucose metabolism and vascular function on late-life cognitive performance in individuals with type 2 diabetes.

\section{... focus on dementia}

In this thesis, we have focused on subtle impairments in cognitive performance rather than on dementia risk. Future research should also focus on the question of whether glucose metabolism and vascular function explain the diabetes-associated increased dementia risk, as it has been suggested that diabetes-associated cognitive decrements and the increased dementia risk in diabetes not necessarily form a continuum. This is 
of particular interest because dementia profoundly affects a person's autonomy and independence.

... go where the money is

The blood-brain barrier separates the central nervous system from the systemic circulation, thereby hampering the use of systemic markers to reflect cerebral glucose metabolism. Ideally, future studies on the role of glucose metabolism in (diabetesassociated) cognitive problems should thus try to assess local metabolism, within the brain itself or the surrounding cerebrospinal fluid. Recent advances in cerebral imaging, such as positron emission tomography and magnetoencephalography, have opened new doors for the assessment of insulin effects in the central nervous system, ${ }^{85}$ and may, perhaps, replace, invasive cerebrospinal fluid sampling as the only alternative.

... open Pandora's box

Of the variables investigated in the present thesis, none explained a substantial part of the variance in cognitive performance among individuals with type 2 diabetes. Hence, there is a clear need to identify factors that do explain why only a subset of individuals with type 2 diabetes will experience cognitive problems. Factors of potential interest are low-grade information, abnormalities in the hypothalamic-pituitary-adrenal axis, and microvascular changes. ${ }^{2,86}$

\section{Conclusion}

Diabetes-associated cognitive problems have become an increasing problem in recent years, with a high impact on patients and society. The present thesis provides evidence that abnormalities in glucose metabolism and vascular function may contribute to the onset and, to a far lesser extent, the severity of these problems. Given that we identified hyperglycaemia as the primary mediator of diabetes-associated differences in cognitive performance, we could propose that diabetes-associated cognitive problems are a glucose-mediated complication of diabetes. The effects of long-term exposure to elevated blood pressure are, however, not to be underestimated, particularly because they might exacerbate the negative cognitive effects of hyperglycaemia. Our work constitutes a first step in developing more rational treatment strategies and suggests that early improvement of glycaemic control and the vascular risk profile may prevent, and maybe even somewhat ameliorate, cognitive problems in diabetes. Although this warrants further study, it has the potential to reduce the individual and societal burden of diabetes-associated problems, thereby enriching the life of those who have diabetes. Would it not be great if all people with diabetes could remember their sweet memories? 


\section{References}

1. Koekkoek, P.S., et al., Cognitive function in patients with diabetes mellitus: guidance for daily care. Lancet Neurol, 2015. 14(3): p. 329-40.

2. Biessels, G.J., et al., Dementia and cognitive decline in type 2 diabetes and prediabetic stages: towards targeted interventions. Lancet Diabetes Endocrinol, 2014. 2(3): p. 246-55.

3. Oeppen, J. and J.W. Vaupel, Demography. Broken limits to life expectancy. Science, 2002. 296(5570): p. 1029-31.

4. Nationaal Kompas. Levensverwachting: achtergrondcijfers. 2012. Available from: http:// www.nationaalkompas.nl/gezondheid-en-ziekte/sterfte-levensverwachting-en-daly-s/ levensverwachting/wat-is-in-nederland-de-levensverwachting/. Last accessed February 12, 2016.

5. Ioacara, S., et al., Improvements in life expectancy in adult type 2 diabetes patients in the last six decades. Diabetes Res Clin Pract, 2011. 92(3): p. 400-4.

6. Mokdad, A.H., et al., Diabetes trends in the U.S.: 1990-1998. Diabetes Care, 2000. 23(9): p. 1278-83.

7. Nationaal Kompas. Diabetes mellitus: incidentie, prevalentie en sterfte naar leeftijd en geslacht. 2011. Available from: http://www.nationaalkompas.nl/gezondheid-en-ziekte/ziekten-enaandoeningen/endocriene-voedings-en-stofwisselingsziekten-en-immuniteitsstoornissen/ diabetes-mellitus/omvang/. Last accessed February 12, 2016.

8. Kahn, S.E., M.E. Cooper, and S. Del Prato, Pathophysiology and treatment of type 2 diabetes: perspectives on the past, present, and future. Lancet, 2014. 383(9922): p. 1068-83.

9. Brownlee, M., Biochemistry and molecular cell biology of diabetic complications. Nature, 2001. 414(6865): p. 813-20.

10. Biessels, G.J., et al., Risk of dementia in diabetes mellitus: a systematic review. Lancet Neurol, 2006. 5(1): p. 64-74.

11. Gispen, W.H. and G.J. Biessels, Cognition and synaptic plasticity in diabetes mellitus. Trends Neurosci, 2000. 23(11): p. 542-9.

12. Sima, A.A., H. Kamiya, and Z.G. Li, Insulin, C-peptide, hyperglycemia, and central nervous system complications in diabetes. Eur J Pharmacol, 2004. 490(1-3): p. 187-97.

13. Cholerton, B., L.D. Baker, and S. Craft, Insulin, cognition, and dementia. Eur J Pharmacol, 2013. 719(1-3): p. 170-9.

14. Schena, F.P. and L. Gesualdo, Pathogenetic mechanisms of diabetic nephropathy. J Am Soc Nephrol, 2005. 16 Suppl 1: p. S30-3.

15. Antonetti, D.A., et al., Diabetic retinopathy: seeing beyond glucose-induced microvascular disease. Diabetes, 2006. 55(9): p. 2401-11.

16. Wallace, T.M., J.C. Levy, and D.R. Matthews, Use and abuse of HOMA modeling. Diabetes Care, 2004. 27(6): p. 1487-95.

17. Van den Berg, E., Y.D. Reijmer, and G.J. Biessels, Diabetes and the Brain; Cognition in Type 2 Dlabetes or Pre-diabetic stages. 1st ed. Comptempary Diabetes, ed. A. Veves. 2010, New York, NY: Humana Press.

18. Craft, S., Insulin resistance syndrome and Alzheimer disease: pathophysiologic mechanisms and therapeutic implications. Alzheimer Dis Assoc Disord, 2006. 20(4): p. 298-301.

19. Schioth, H.B., et al., Brain insulin signaling and Alzheimer's disease: current evidence and future directions. Mol Neurobiol, 2012. 46(1): p. 4-10.

20. Chen, Y., et al., Deregulation of brain insulin signaling in Alzheimer's disease. Neurosci Bull, 2014. 30(2): p. 282-94.

21. Kern, W., et al., Low cerebrospinal fluid insulin levels in obese humans. Diabetologia, 2006. 49(11): p. 2790-2.

22. Colosia, A.D., R. Palencia, and S. Khan, Prevalence of hypertension and obesity in patients with type 2 diabetes mellitus in observational studies: a systematic literature review. Diabetes 
Metab Syndr Obes, 2013. 6: p. 327-38.

23. Mooradian, A.D., Dyslipidemia in type 2 diabetes mellitus. Nat Clin Pract Endocrinol Metab, 2009. 5(3): p. 150-9.

24. Ahtiluoto, S., et al., Diabetes, Alzheimer disease, and vascular dementia: a population-based neuropathologic study. Neurology, 2010. 75(13): p. 1195-202.

25. Peila, R., et al., Type 2 diabetes, APOE gene, and the risk for dementia and related pathologies: The Honolulu-Asia Aging Study. Diabetes, 2002. 51(4): p. 1256-62.

26. MacKnight, C., et al., Diabetes mellitus and the risk of dementia, Alzheimer's disease and vascular cognitive impairment in the Canadian Study of Health and Aging. Dement Geriatr Cogn Disord, 2002. 14(2): p. 77-83.

27. Fontbonne, A., et al., Changes in cognitive abilities over a 4-year period are unfavorably affected in elderly diabetic subjects: results of the Epidemiology of Vascular Aging Study. Diabetes Care, 2001. 24(2): p. 366-70.

28. Kanaya, A.M., et al., Change in cognitive function by glucose tolerance status in older adults: a 4-year prospective study of the Rancho Bernardo study cohort. Arch Intern Med, 2004. 164(12): p. 1327-33.

29. Asimakopoulou, K.G., S.E. Hampson, and N.J. Morrish, Neuropsychological functioning in older people with type 2 diabetes: the effect of controlling for confounding factors. Diabet Med, 2002. 19(4): p. 311-6.

30. Gregg, E.W., et al., Is diabetes associated with cognitive impairment and cognitive decline among older women? Study of Osteoporotic Fractures Research Group. Arch Intern Med, 2000. 160(2): p. 174-80.

31. McFall, G.P., et al., Testing covariates of Type 2 diabetes-cognition associations in older adults: moderating or mediating effects? Neuropsychology, 2010. 24(5): p. 547-62.

32. Van den Berg, E., et al., Type 2 diabetes mellitus, hypertension, dyslipidemia and obesity: A systematic comparison of their impact on cognition. Biochim Biophys Acta, 2009. 1792(5): p. 470-81.

33. Kloppenborg, R.P., et al., Diabetes and other vascular risk factors for dementia: which factor matters most? A systematic review. Eur J Pharmacol, 2008. 585(1): p. 97-108.

34. Sabayan, B., et al., Association of visit-to-visit variability in blood pressure with cognitive function in old age: prospective cohort study. BMJ, 2013. 347: p. f4600.

35. Stehouwer, C.D., R.M. Henry, and I. Ferreira, Arterial stiffness in diabetes and the metabolic syndrome: a pathway to cardiovascular disease. Diabetologia, 2008. 51(4): p. 527-39.

36. Henry, R.M., et al., Carotid arterial remodeling: a maladaptive phenomenon in type 2 diabetes but not in impaired glucose metabolism: the Hoorn study. Stroke, 2004. 35(3): p. 671-6.

37. Henry, R.M., et al., Arterial stiffness increases with deteriorating glucose tolerance status: the Hoorn Study. Circulation, 2003. 107(16): p. 2089-95.

38. Kozakova, M., et al., Glucose-related arterial stiffness and carotid artery remodeling: a study in normal subjects and type 2 diabetes patients. J Clin Endocrinol Metab, 2014. 99(11): p. E2362-6.

39. Mitchell, G.F., et al., Arterial stiffness, pressure and flow pulsatility and brain structure and function: the Age, Gene/Environment Susceptibility--Reykjavik study. Brain, 2011. 134(Pt 11): p. 3398-407.

40. O'Rourke, M.F. and M.E. Safar, Relationship between aortic stiffening and microvascular disease in brain and kidney: cause and logic of therapy. Hypertension, 2005. 46(1): p. 200-4.

41. Van Sloten, T.T., et al., Association between arterial stiffness, cerebral small vessel disease and cognitive impairment: A systematic review and meta-analysis. Neurosci Biobehav Rev, 2015. 53: p. 121-30.

42. Feinkohl, I., et al., The impact of diabetes on cognitive decline: potential vascular, metabolic, and psychosocial risk factors. Alzheimers Res Ther, 2015. 7(1): p. 46.

43. Fioretto, P., et al., Renal protection in diabetes: role of glycemic control. J Am Soc Nephrol, 
2006. 17(4 Suppl 2): p. S86-9.

44. Calcutt, N.A., et al., Therapies for hyperglycaemia-induced diabetic complications: from animal models to clinical trials. Nat Rev Drug Discov, 2009. 8(5): p. 417-29.

45. Nathan, D.M., et al., Intensive diabetes treatment and cardiovascular disease in patients with type 1 diabetes. N Engl J Med, 2005. 353(25): p. 2643-53.

46. Ceriello, A., M.A. Ihnat, and J.E. Thorpe, Clinical review 2: The "metabolic memory": is more than just tight glucose control necessary to prevent diabetic complications? J Clin Endocrinol Metab, 2009. 94(2): p. 410-5.

47. Lamport, D.J., et al., Impairments in glucose tolerance can have a negative impact on cognitive function: a systematic research review. Neurosci Biobehav Rev, 2009. 33(3): p. 394-413.

48. Matthaei, S., R. Horuk, and J.M. Olefsky, Blood-brain glucose transfer in diabetes mellitus. Decreased number of glucose transporters at blood-brain barrier. Diabetes, 1986. 35(10): p. 1181-4.

49. Pardridge, W.M., D. Triguero, and C.R. Farrell, Downregulation of blood-brain barrier glucose transporter in experimental diabetes. Diabetes, 1990. 39(9): p. 1040-4.

50. Biessels, G.J. and Y.D. Reijmer, Brain changes underlying cognitive dysfunction in diabetes: what can we learn from MRI? Diabetes, 2014. 63(7): p. 2244-52.

51. Chantler, P.D., et al., Cerebral Cortical Microvascular Rarefaction in Metabolic Syndrome is Dependent on Insulin Resistance and Loss of Nitric Oxide Bioavailability. Microcirculation, 2015. 22(6): p. 435-45.

52. Mooradian, A.D., Central nervous system complications of diabetes mellitus--a perspective from the blood-brain barrier. Brain Res Brain Res Rev, 1997. 23(3): p. 210-8.

53. Last, D., et al., Global and regional effects of type 2 diabetes on brain tissue volumes and cerebral vasoreactivity. Diabetes Care, 2007. 30(5): p. 1193-9.

54. Reijmer, Y.D., et al., Development of vascular risk factors over 15 years in relation to cognition: the Hoorn Study. J Am Geriatr Soc, 2012. 60(8): p. 1426-33.

55. Elias, P.K., et al., NIDDM and blood pressure as risk factors for poor cognitive performance. The Framingham Study. Diabetes Care, 1997. 20(9): p. 1388-95.

56. Hassing, L.B., et al., Comorbid type 2 diabetes mellitus and hypertension exacerbates cognitive decline: evidence from a longitudinal study. Age Ageing, 2004. 33(4): p. 355-61.

57. Xu, W.L., et al., Diabetes mellitus and risk of dementia in the Kungsholmen project: a 6-year follow-up study. Neurology, 2004. 63(7): p. 1181-6.

58. Stern, Y., et al., Exploring the neural basis of cognitive reserve. J Clin Exp Neuropsychol, 2003. 25(5): p. 691-701.

59. Hall, P.M., Prevention of Progression in Diabetic Nephropathy. Diabetes Spectrum, 2006. 19(1): p. 18-24.

60. Chatterjee, S., et al., Type 2 Diabetes as a Risk Factor for Dementia in Women Compared With Men: A Pooled Analysis of 2.3 Million People Comprising More Than 100,000 Cases of Dementia. Diabetes Care, 2016. 39(2): p. 300-7.

61. Banks, W.A., J.B. Owen, and M.A. Erickson, Insulin in the brain: there and back again. Pharmacol Ther, 2012. 136(1): p. 82-93.

62. Tschritter, O., et al., Insulin effects on beta and theta activity in the human brain are differentially affected by ageing. Diabetologia, 2009. 52(1): p. 169-71.

63. Twig, G., et al., Cognitive function and the risk for diabetes among young men. Diabetes Care, 2014. 37(11): p. 2982-8.

64. Cole, D.A. and S.E. Maxwell, Testing mediational models with longitudinal data: questions and tips in the use of structural equation modeling. J Abnorm Psychol, 2003. 112(4): p. 558-77.

65. Maxwell, S.E. and D.A. Cole, Bias in cross-sectional analyses of longitudinal mediation. Psychol Methods, 2007. 12(1): p. 23-44.

66. Schisterman, E.F., S.R. Cole, and R.W. Platt, Overadjustment bias and unnecessary adjustment in epidemiologic studies. Epidemiology, 2009. 20(4): p. 488-95. 
67. Biessels, G.J., I.J. Deary, and C.M. Ryan, Cognition and diabetes: a lifespan perspective. Lancet Neurol, 2008. 7(2): p. 184-90.

68. Kessels, R.P. and A.M. Brands, Diabetes and the Brain; Neuropsychological Assessment. 1st ed. Comptempary Diabetes, ed. A. Veves. 2010, New York, NY: Humana Press.

69. Palta, P., et al., Magnitude of cognitive dysfunction in adults with type 2 diabetes: a metaanalysis of six cognitive domains and the most frequently reported neuropsychological tests within domains. J Int Neuropsychol Soc, 2014. 20(3): p. 278-91.

70. Brands, A.M., et al., A detailed profile of cognitive dysfunction and its relation to psychological distress in patients with type 2 diabetes mellitus. J Int Neuropsychol Soc, 2007. 13(2): p. 28897.

71. McCrimmon, R.J., C.M. Ryan, and B.M. Frier, Diabetes and cognitive dysfunction. Lancet, 2012. 379(9833): p. 2291-9.

72. Lezak, M.D., et al., Neuropsychological Assessment. 2012: OUP USA.

73. DeFronzo, R.A., J.D. Tobin, and R. Andres, Glucose clamp technique: a method for quantifying insulin secretion and resistance. Am J Physiol, 1979. 237(3): p. E214-23.

74. Cersosimo, E., et al., Assessment of pancreatic beta-cell function: review of methods and clinical applications. Curr Diabetes Rev, 2014. 10(1): p. 2-42.

75. DeFronzo, R.A., Pharmacologic therapy for type 2 diabetes mellitus. Ann Intern Med, 2000. 133(1): p. 73-4.

76. American Diabetes Association. Standards of medical care in diabetes - 2016. Diabetes Care, 2016. 39(Supplement 1): p. S1-S112.

77. Garber, A.J., et al., Consensus Statement by the American Association of Clinical Endocrinologists and American College of Endocrinology on the Comprehensive Type 2 Diabetes Management Algorithm - 2016 Executive Summary. Endocr Pract, 2016. 22(1): p. 84-113.

78. Lily, M. and M. Godwin, Treating prediabetes with metformin: systematic review and metaanalysis. Can Fam Physician, 2009. 55(4): p. 363-9.

79. Lind, M., et al., The association between HbA1c, fasting glucose, 1-hour glucose and 2-hour glucose during an oral glucose tolerance test and cardiovascular disease in individuals with elevated risk for diabetes. PLoS One, 2014. 9(10): p. e109506.

80. Cukierman-Yaffe, T., et al., Effects of basal insulin glargine and omega-3 fatty acid on cognitive decline and probable cognitive impairment in people with dysglycaemia: a substudy of the ORIGIN trial. Lancet Diabetes Endocrinol, 2014. 2(7): p. 562-72.

81. Koekkoek, P.S., et al., Intensive multifactorial treatment and cognitive functioning in screendetected type 2 diabetes--the ADDITION-Netherlands study: a cluster-randomized trial. J Neurol Sci, 2012. 314(1-2): p. 71-7.

82. Launer, L.J., et al., Effects of intensive glucose lowering on brain structure and function in people with type 2 diabetes (ACCORD MIND): a randomised open-label substudy. Lancet Neurol, 2011. 10(11): p. 969-77.

83. De Galan, B.E., et al., Cognitive function and risks of cardiovascular disease and hypoglycaemia in patients with type 2 diabetes: the Action in Diabetes and Vascular Disease: Preterax and Diamicron Modified Release Controlled Evaluation (ADVANCE) trial. Diabetologia, 2009. 52(11): p. 2328-36.

84. Williamson, J.D., et al., Cognitive function and brain structure in persons with type 2 diabetes mellitus after intensive lowering of blood pressure and lipid levels: a randomized clinical trial. JAMA Intern Med, 2014. 174(3): p. 324-33.

85. Tschritter, O., et al., The cerebrocortical response to hyperinsulinemia is reduced in overweight humans: a magnetoencephalographic study. Proc Natl Acad Sci U S A, 2006. 103(32): p. 12103-8. 
86. Heringa, S.M., et al., Associations between retinal microvascular changes and dementia, cognitive functioning, and brain imaging abnormalities: a systematic review. J Cereb Blood Flow Metab, 2013. 33(7): p. 983-95. 
NEDERLANDSTALIGE SAMENVATTING 


\section{Nederlandstalige samenvatting}

Naar schatting lijdt één miljoen Nederlanders aan diabetes mellitus, oftewel suikerziekte. Diabetes ontstaat door een absoluut of relatief tekort aan insuline, het hormoon dat een belangrijke rol speelt bij het transport van glucose uit het bloed naar de weefsels en lichaamscellen. Het resultaat is een verhoogde bloedglucosespiegel (bloedsuiker) die op korte termijn veelal weinig klachten geeft en onopgemerkt kan blijven. Dit is dan ook de reden dat een kwart van de Nederlanders die diabetes heeft dit zelf nog niet weet. Toch kunnen de gevolgen desastreus zijn. Het is bekend dat langdurige blootstelling aan verhoogde bloedglucosespiegels kan leiden tot chronische schade aan de ogen (retinopathie), nieren (nefropathie) en bloedvaten (hart- en vaatziekten). Mensen met diabetes worden dan ook frequent, veelal door hun huisarts, gecontroleerd op het ontwikkelen van deze zogenoemde chronische complicaties.

De afgelopen decennia, en zeker de laatste jaren, is er veel belangstelling voor de mogelijke effecten van diabetes op het brein. Hoewel glucose de belangrijkste brandstof is voor het brein, laat recent onderzoek zien dat mensen met type 2 diabetes, de meest voorkomende vorm van suikerziekte, een tweemaal verhoogd risico hebben op het ontwikkelen van dementie in vergelijking met mensen zonder diabetes. Maar niet alleen dementie komt vaker voor. Mensen met diabetes scoren in het algemeen lager op een breed scala aan cognitieve testen. Er is dus sprake van aantasting van de cognitieve functies, de processen die betrokken zijn bij het verwerven en verwerken van informatie. Voorbeelden van cognitieve functies zijn geheugen, aandacht en concentratie, snelheid van informatieverwerking, planningsvaardigheid en probleemoplossend vermogen. Dit betekent dat mensen met diabetes moeite kunnen hebben met het herinneren van recente gebeurtenissen, het uitvoeren van complexe taken en het nemen van beslissingen. En dan hebben we het niet eens over de invaliderende effecten van dementie waarbij het normale dagelijkse functioneren van personen (volledig) is verstoord.

Eerder onderzoek heeft dus aangetoond dat diabetes ook in het brein zijn sporen achterlaat: lagere cognitieve prestaties en een verhoogd risico op dementie. Toch blijft de vraag hoe we deze negatieve gevolgen van diabetes op het brein kunnen verklaren. Het antwoord op deze vraag is belangrijk omdat steeds meer mensen te maken krijgen met de schadelijke effecten van diabetes. Dit komt niet alleen door de aanhoudende obesitas problematiek die het ontwikkelen van diabetes in de hand werkt, maar ook omdat de levensverwachting van mensen met diabetes sterk is verbeterd. Dit proefschrift beschrijft een aantal studies met als collectief doel meer inzicht te krijgen in de factoren die bijdragen aan het ontstaan van aan diabetes gerelateerde cognitieve problemen, alsmede de factoren die bepalend zijn voor hun ernst. We hebben ons hierbij gericht 
op twee kandidaat-factoren: glucose metabolisme en het functioneren van de grotere bloedvaten (vasculaire functie).

\section{Glucose metabolisme}

Diabetes wordt gekenmerkt door verhoogde bloedglucosespiegels (hyperglykemie) en ongevoeligheid van het lichaam voor insuline (insulineresistentie). Er zijn meerdere manieren waarop hyperglykemie en insulineresistentie negatieve effecten kunnen hebben op het brein. Zo veroorzaakt hyperglykemie oxidatieve stress, een complex proces waarbij zogenoemde vrije radicalen vrijkomen die (zenuw)cellen onherstelbaar kunnen beschadigen. Daarnaast is bekend dat insuline een wisselwerking heeft op tau en amyloid- $\beta$, twee eiwitten die een belangrijke rol spelen bij het ontstaan van Alzheimer dementie. Het is dan ook niet vreemd om te denken dat de afwijkingen in het glucose metabolisme die maken dat iemand diabetes heeft ook verklaren waarom mensen met diabetes lager scoren op cognitieve taken dan mensen zonder diabetes. En hoe voordehandliggend dan ook, niet eerder werd onderzocht of hyperglykemie en insulineresistentie inderdaad diabetesgerelateerde cognitieve problemen verklaren. Wij onderzochten dit in De Maastricht Studie, een groot onderzoek uitgevoerd in de regio Maastricht (Hoofdstuk 2). Onze resultaten, gebaseerd op de gegevens van 2531 deelnemers waaronder 666 deelnemers met type 2 diabetes, lieten zien dat verschillen in de snelheid van informatieverwerking en uitvoerende functies, waaronder planning en oplossend vermogen, voor 50 tot $80 \%$ verklaard worden door hyperglykemie. Deze resultaten suggereren dat hyperglykemie een belangrijke factor kan zijn bij het ontstaan van aan diabetes gerelateerde cognitieve problemen. Insulineresistentie daarentegen verklaarde niet waarom mensen met diabetes cognitief slechter presteerden dan mensen met een normaal glucose metabolisme en lijkt dan ook geen (belangrijke) rol te spelen bij het ontstaan van aan diabetes gerelateerde cognitieve problemen.

Gelukkig kampt niet iedereen met diabetes met cognitieve problemen. De volgende vraag is dan ook of hyperglykemie en insulineresistentie de variatie in cognitief functioneren binnen mensen met diabetes kunnen verklaren. Om antwoord te vinden op deze vraag verrichtten we een literatuurstudie (Hoofdstuk 5). De resultaten van 86 eerdere studies lieten zien dat er inderdaad een relatie bestaat tussen hyperglykemie en het cognitief functioneren van mensen met type 2 diabetes. Vooral een hoger $\mathrm{HbA} 1 \mathrm{c}$ gehalte, een maat die de gemiddelde bloedglucosespiegel van de afgelopen 8 tot 12 weken weerspiegelt, bleek samen te hangen met slechter cognitief functioneren. De sterke van deze relatie was echter zwak: hyperglykemie kon veelal niet meer dan $10 \%$ van de variatie in cognitief functioneren binnen mensen met type 2 diabetes verklaren. Uit onze literatuurstudie bleek ook de noodzaak voor verder onderzoek naar de rol van insulineresistentie omdat er te weinig studies waren om een betrouwbaar 
antwoord te geven op de vraag of insulineresistentie een factor is die de variatie in cognitie tussen mensen met diabetes verklaart. Opnieuw maakten we gebruik van De Maastricht Studie, ditmaal van de gegevens van 806 deelnemers met type 2 diabetes die allemaal een uitgebreide batterij een cognitieve testen voltooiden (Hoofdstuk 6). Maten van insulineresistentie bleken niet samen te hangen met cognitief functioneren in deze groep. Gecombineerd met onze literatuurstudie suggereren deze resultaten dat hyperglykemie, maar niet insulineresistentie, bijdraagt, hetzij in beperkte mate, aan de ernst van diabetesgerelateerde cognitieve problemen.

Mogen we dan concluderen dat insulineresistentie geen enkel effect heeft op het menselijk brein terwijl proefdierstudies in toenemende mate laten zien dat insulineresistentie samenhangt met het ontwikkelen van Alzheimer dementie? Waarschijnlijk niet. Cerebrale insulineresistentie (ongevoeligheid van het brein voor insuline) is niet rechtstreeks te meten in levende mensen. Veelal wordt daarom de insulinespiegel in het bloed gebruikt om de mate van insulineresistentie te schatten. Zo ook in De Maastricht Studie (Hoofdstukken 2 en 6). Hier kleven echter nadelen aan. De insulinespiegel in het bloed is niet noodzakelijkerwijze gelijk aan de insulinespiegel in het brein. Dit komt omdat insuline actief getransporteerd wordt over de bloed-hersenbarrière, de letterlijke grens tussen het bloed en de hersenen, en dit transport onder invloed van veel factoren staat. Daar komt bij dat recent onderzoek laat zien dat de mate van insulineresistentie in het brein kan verschillen met die in de rest van het lichaam. Insulinespiegels in het cerebrospinale vocht, het vocht dat de hersenen en het ruggenmerg omringd, corresponderen waarschijnlijk beter met de werkelijke insulinespiegels in het brein, en dus met de mate van cerebrale insulineresistentie. Onderzoeksgegevens van de Parel Neurodegeneratieve Ziekten, onderdeel van het Parelsnoer Initiatief, een uniek samenwerkingsverband tussen alle academische centra in Nederland, maakten het mogelijk om de relatie tussen insulinespiegels in de cerebrospinale vloeistof en cognitief functioneren in kaart te brengen (Hoofdstuk 7). Dit onderzoek, verricht onder 138 individuen met geheugenproblemen, liet zien dat hogere insulinespiegels samenhingen met lagere cognitieve prestaties en hogere spiegels van het tau-eiwit, zoals eerder aangegeven een eiwit dat betrokken is bij het ontstaan van Alzheimer dementie. Dit suggereert dat insulineresistentie in het brein toch negatieve effecten kan hebben op de breinfunctie, hoewel de gevonden relaties enkel aanwezig waren in bepaalde groepen mensen (vrouwen en niet-dragers van het zogenoemde Alzheimer gen, apolipoproteïne E ع4). Een mogelijke verklaring voor deze bevinding is dat insulinegevoeligheid van deze groepen mensen anders is dan die van de rest.

\section{Vasculaire functie}

Mensen met diabetes hebben vaak ook andere vasculaire risicofactoren. Zo heeft 
naar schatting 70 tot $75 \%$ van de mensen met type 2 diabetes een te hoge bloeddruk (hypertensie) en een te hoog cholesterol (hypercholesterolemie). Elk van deze risicofactoren afzonderlijk wordt in de literatuur in verband gebracht met slechter cognitief functioneren. Het is dan ook niet verwonderlijk dat vaak wordt aangenomen dat aan diabetes gerelateerde cognitieve problematiek voor een deel is toe te schrijven aan de aanwezigheid van deze bijkomende vasculaire risicofactoren. Deze gedachte wordt ondersteund door autopsie-studies die laten zien dat het brein van overleden mensen met type 2 diabetes meer vasculaire schade laat zien dat het brein van overleden mensen zonder diabetes. Toch slaagden eerdere epidemiologische studies (populatiestudies) er niet in deze gedachtegang te bevestigen dan wel te ontkrachten. Met behulp van gegevens van De Maastricht Studie hebben wij getracht dichter bij een antwoord te komen op de vraag of traditionele vasculaire risicofactoren, en dan met name bloeddruk, een rol spelen bij het ontstaan van aan diabetes gerelateerde cognitieve problemen (Hoofdstuk 2). De resultaten, gebaseerd op de gegevens van 2531 deelnemers, lieten zien dat de verschillen in snelheid van informatieverwerking tussen mensen met type 2 diabetes en mensen met een normaal glucose metabolisme voor ongeveer $20 \%$ verklaard worden door bloeddruk. Hierbij blijkt met name een rol weggelegd voor het gebruik van bloeddrukverlagende medicatie en dus waarschijnlijk vroegere blootstelling aan hypertensie. Andere traditionele risicofactoren zoals de buikomvang en het doorgemaakt hebben van hart- en vaatziekten verklaarden nauwelijks de cognitieve verschillen tussen mensen met en zonder diabetes.

Naast bovengenoemde traditionele vasculaire risicofactoren is er toenemend aandacht voor het functioneren van de (grotere) vaten. Eerder onderzoek heeft aangetoond dat vaatdisfunctie, waaronder verstijving van de bloedvaten, kan leiden tot cognitieve achteruitgang. Gedacht wordt dat bij stijvere bloedvaten het bloed met een grotere kracht het brein bereikt en daardoor de kleinste bloedvaten in het brein makkelijk kan beschadigen. Een interessant gegeven is dat recent onderzoek ook laat zien dat diabetes het proces van vaatverstijving kan versnellen, wat de vraag doet oproepen of vaatstijfheid een verklarende factor kan zijn voor aan diabetes gerelateerde cognitieve problemen. Ook deze vraag kon met behulp van data van De Maastricht Studie worden beantwoord, omdat deelnemers aan de studie een uitgebreid echografisch onderzoek ondergingen om de structuur en functie van hun bloedvaten in kaart te brengen. Lokale stijfheid van de halsslagader bleek samen te hangen met verminderd cognitief functioneren, zowel bij mensen met als zonder diabetes, maar verklaarde niet de gevonden verschillen in cognitief functioneren tussen mensen met en zonder type 2 diabetes (Hoofdstuk 3). Ook keken we naar mate van stress op de wand van de halsslagader, een mogelijke andere maat voor vaatfunctie daar de wandstress onder normale omstandigheden door structurele aanpassingen van de bloedvaten constant wordt gehouden (Hoofdstuk 4). 
Hoewel een verhoogde circumferentiële wandstress in verband wordt gebracht met een verhoogd risico op vasculaire schade in het brein, konden wij geen relatie aantonen tussen wandstress enerzijds en cognitief functioneren anderzijds. Vergelijkbare resultaten werden gevonden wanneer mensen met en zonder type 2 diabetes los van elkaar werden onderzocht. De resultaten van dit proefschrift suggereren dus dat vasculaire functie en abnormaliteiten slechts in beperkte mate bijdragen aan het ontstaan van aan diabetes gerelateerde cognitieve problemen.

Geldt dat dan ook voor de variatie in cognitief functioneren binnen mensen met type 2 diabetes? Een eerdere literatuurstudie van onderzoekers uit het Verenigd Koninkrijk doet vermoeden van wel, althans zij concludeerden dat de bijdrage van vasculaire factoren aan de ernst van diabetes gerelateerde problematiek discutabel is. Onze bevindingen laten zien dat traditionele vasculaire risicofactoren waaronder de buikomvang, cholesterolspiegel, bloeddruk en roken tezamen niet meer dan $2.5 \%$ van de variatie in cognitief functioneren binnen mensen met diabetes verklaren (Hoofdstuk 6). Bovendien, hoewel we een relatie aantoonden tussen vaatstijfheid van de halsslagader en cognitief functioneren bij mensen met type 2 diabetes (Hoofdstuk 3), was de sterke van dit verband beperkt, hetgeen het weinig waarschijnlijk maakt dat vaatstijfheid in grote mate bepalend is voor de variatie in cognitie binnen mensen met diabetes.

\section{Conclusie}

Dit proefschrift had tot doel meer inzicht te krijgen in de factoren die bijdragen aan het ontstaan van aan diabetes gerelateerde cognitieve problemen, alsmede de factoren die bepalend zijn voor hun ernst. We kunnen concluderen dat zowel hyperglykemie (in meerdere mate) als vasculaire factoren (in mindere mate) bijdragen aan de verschillen in cognitief functioneren tussen mensen met en zonder type 2 diabetes, maar tegelijkertijd slechts zeer beperkt verklaren waarom cognitieve prestaties binnen mensen met diabetes verschillen. Onze resultaten suggereren dat aan diabetes gerelateerde cognitieve problematiek beschouwd kan worden als een glucose-gemedieerde complicatie van type 2 diabetes en biedt daarmee ook handvatten voor primaire preventie van diabetes gerelateerde cognitieve problemen. 
Nederlandstalige samenvatting 
VALORISATION ADDENDUM 


\section{Valorisation addendum}

Creating knowledge through research seems pointless when this new knowledge does not reach those who might benefit from it. Knowledge valorisation, ie, translation of research into practice, is an essential step in making research worthwhile. Here we discuss how the results of this thesis may impact society. To make it more lively, we start by introducing Mrs. Janssen.

Mrs. Janssen is a 65-year-old woman, slightly overweight and mild hypertensive, who visits her general practitioner for her annual diabetes check-up. She was diagnosed with type 2 diabetes 10 years ago and her glycaemic control has been adequate, though not perfect, ever since. Normally, she would not be nervous to visit her general practitioner, but today she is. She recently participated in The Maastricht Study where she was subjected, among many other things, to a cognitive test battery. When she asked the examiner who conducted the cognitive assessment why this test battery was administered, the friendly PhD student told her that people with type 2 diabetes have an increased risk of developing dementia and other cognitive problems. Although Mrs. Jansen knew that she had been free from diabetic complications, she was afraid that the diabetes might have affected her brain. She frequently forgot names and had to keep a diary to remind herself of the appointments she made. She asked herself: "do these complaints indicate that I am suffering from diabetes-associated cognitive problems?", "how can I prevent myself from developing diabetes-associated cognitive problems?", and "are there any treatment options available for diabetes-associated cognitive problems?". With these questions in mind, she asked her husband, a 63-year-old obese man with prediabetes, to join her to the general practitioner.

\section{Awareness}

Many people with type 2 diabetes do not know that their disease puts them at increased risk of cognitive problems. If this thesis succeeds in raising this awareness, especially among patients and caregivers, it would already be of societal value. After all, awareness is the first step towards early detection, treatment and perhaps even prevention of diabetes-associated cognitive problems. One might, however, argue that increasing this awareness is somewhat unethical as it might scare people while there is, at least at present, no treatment available that can alter the course of cognitive impairment. Moreover, it is difficult to establish to what extent the subtle cognitive decrements often seen in people with diabetes will impact their daily lives and well-being. Yet, it has been shown that individuals with type 2 diabetes with undiagnosed cognitive impairment do suffer from more depressive symptoms and have a lower health status. ${ }^{1}$ In addition, cognitive problems clearly impact diabetes self-care, which results in worse glycaemic 
control and a higher likelihood of hypoglycaemia.

\section{Novel insights}

To understand diabetes-associated cognitive problems, one must know the factors that contribute to their onset and severity. This thesis is an effort to identify such factors and suggests that diabetes-associated cognitive problems should be considered as a glucose-mediated complication of type 2 diabetes, since the differences in cognitive performance between individuals with and without diabetes were largely explained by hyperglycaemia. The effects of long-term exposure to elevated blood pressure should, however, not be overlooked as they may also, to a lesser extent, contribute to the cognitive sequelae of diabetes, and could exacerbate the negative cognitive effects of hyperglycaemia. Importantly, however, none of the metabolic and vascular factors we explored explained a large amount of the variance in cognitive performance among individuals with type 2 diabetes. Abnormalities in glucose metabolism and vascular function are thus less likely to explain why only a subset of individuals with type 2 diabetes experience cognitive problems.

\section{Prevention targets}

Based on the findings of this thesis, we believe that early glycaemic and blood pressure control might be promising targets for the primary prevention of diabetes-associated cognitive problems. At present, individuals with prediabetes are encouraged to change their lifestyle in order to lose weight and hopefully return to normal glucose metabolism. ${ }^{2}$ ${ }^{3}$ Guidelines also recommend the identification, and if appropriate, treatment of other vascular risk factors, including hypertension. There, are, however no strict targets for glycaemic control and individuals with prediabetes are seldom prescribed glucoselowering medication to prevent deterioration in glycaemic control. ${ }^{2}$ Our work indicates that intensified glycaemic and blood pressure control in those without diabetes may be necessary to prevent diabetes-associated cognitive problems. This adds fuel to the debate as to whether individuals with prediabetes should be treated with glucoselowering treatment. The prevention of cognitive problems may be just another reason to consider targets for glycaemic control in people without diabetes. This view is supported by results of the Outcome Reduction with Initial Glargine Intervention (ORIGIN) trial, which found that insulin therapy in prediabetes to normalise blood glucose levels tends to reduce the decline in some aspects of cognitive performance. ${ }^{4}$

People who already have diabetes do not benefit from primary prevention. For them, secondary prevention of diabetes-associated cognitive problems is necessary, that is, amelioration of cognitive problems among individuals with type 2 diabetes. Unfortunately, our thesis provides little guidance on how to target secondary prevention 
as we showed that hyperglycaemia, carotid arterial stiffness, and traditional vascular risk factors explain only marginal amounts of variance in cognitive performance in diabetes. Although this may be why randomised controlled trials on intensified glucoselowering, ${ }^{5-7}$ blood pressure-lowering, ${ }^{8}$ or lipid-lowering ${ }^{8}$ treatment in people with type 2 diabetes have failed to show improvement in cognitive performance, it does not foreclose the possibility that improving the metabolic and vascular risk profile of individuals with diabetes may have some beneficial effects. Indeed, positive effects on cognitive performance were observed in both treatment groups in most randomised trials on intensified glucose-lowering treatment, ${ }^{6,7}$ suggesting that improving glycaemic control does have beneficial effects on brain function.

Back to Mrs. Janssen. Luckily for her, her general practitioner recently received a copy of this thesis and he even found some time to read it. He decides to refer her to a neuropsychologist for detailed neuropsychological assessment, which might help to differentiate between (diabetes-associated) cognitive problems and normal cognitive functioning. He also increases her dose of glucose-lowering and antihypertensive medication, although he knows, and warns Mrs. Janssen, that this is likely to have little or no effect on (the progression of) her cognitive complaints. Then he turns to the 63-yearold obese Mr. Janssen with prediabetes, who has failed every diet over the last 5 years, and starts to wonder whether he should start glucose-lowering treatment. 


\section{References}

1. Koekkoek, P.S., et al., Undiagnosed cognitive impairment, health status and depressive symptoms in patients with type 2 diabetes. J Diabetes Complications, 2015. 29(8): p. 1217-22.

2. American Diabetes Association. Standards of medical care in diabetes - 2016. Diabetes Care, 2016. 39(Supplement 1): p. S1-S112.

3. Garber, A.J., et al., Consensus Statement by the American Association of Clinical Endocrinologists and American College of Endocrinology on the Comprehensive Type 2 Diabetes Management Algorithm - 2016 Executive Summary. Endocr Pract, 2016. 22(1): p. 84-113.

4. Cukierman-Yaffe, T., et al., Effects of basal insulin glargine and omega-3 fatty acid on cognitive decline and probable cognitive impairment in people with dysglycaemia: a substudy of the ORIGIN trial. Lancet Diabetes Endocrinol, 2014. 2(7): p. 562-72.

5. De Galan, B.E., et al., Cognitive function and risks of cardiovascular disease and hypoglycaemia in patients with type 2 diabetes: the Action in Diabetes and Vascular Disease: Preterax and Diamicron Modified Release Controlled Evaluation (ADVANCE) trial. Diabetologia, 2009. 52(11): p. 2328-36.

6. Koekkoek, P.S., et al., Intensive multifactorial treatment and cognitive functioning in screendetected type 2 diabetes--the ADDITION-Netherlands study: a cluster-randomized trial. J Neurol Sci, 2012. 314(1-2): p. 71-7.

7. Launer, L.J., et al., Effects of intensive glucose lowering on brain structure and function in people with type 2 diabetes (ACCORD MIND): a randomised open-label substudy. Lancet Neurol, 2011. 10(11): p. 969-77.

8. Williamson, J.D., et al., Cognitive function and brain structure in persons with type 2 diabetes mellitus after intensive lowering of blood pressure and lipid levels: a randomized clinical trial. JAMA Intern Med, 2014. 174(3): p. 324-33. 


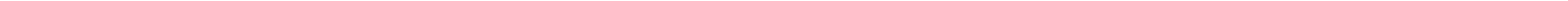


DANKWOORD 


\section{Dankwoord}

Niet alles is wat het lijkt. Op de voorkant van dit proefschrift prijkt één naam. Er zijn echter veel mensen die direct of indirect hebben bijgedragen aan de totstandkoming ervan. Ik ben hen veel dank verschuldigd. Hierbij denk ik onder meer, maar niet uitsluitend, aan de deelnemers van De Maastricht Studie, mijn collega's, alle co-auteurs en de beoordelingscommissie van dit proefschrift. Een paar mensen verdienen mijns inziens een speciaal woord van dank.

Allereerst mijn promotieteam: Coen (Stehouwer), Geert Jan (Biessels) en Simone (Sep). Wat heb ik ontzettend veel geluk gehad dat jullie mij de afgelopen jaren hebben begeleid. Zonder twijfel een dreamteam waar menig collega promovendus jaloers op was.

Coen: de snelheid waarmee jij manuscripten leest, mails beantwoordt en schakelt tussen materie is al meermaals geprezen maar blijft ongekend. Meermaals heb ik me afgevraagd: Hoe doet hij dat? Hoe houdt hij dat vol? Heeft hij nooit behoefte aan een moment van rust of ontspanning? Inmiddels denk ik het patroon te hebben doorgrond: zaterdag een rustdag en de rest van de week non-stop door. Maar toegegeven: ik kan er faliekant naast zitten (patroonherkenning blijft immers lastig). Hoewel ik de snelheid waarmee je werkt enorm bewonder, is het vooral de accuratesse waarmee je dit doet die mij telkens weer verbaasde. Zelfs de kleinste (taalkundige) onjuistheden werden door jou opgemerkt. Je doorziet het grotere geheel maar blijft oog houden voor het kleinste detail. Er zijn maar weinigen die dat goed kunnen. Je bent recht door zee, zegt waar het op staat en dat kan ik ontzettend waarderen. Jouw expertise, perfectionisme en genialiteit werken aanstekelijk: het heeft mij gestimuleerd het uiterste uit mezelf te halen. Af en toe een inhoudelijke discussie aangaan zag ik als een sport, hoewel ik wist dat ik veelal relatief snel schaakmat gezet zou worden. Ik vond het leuk en mooi te bemerken dat er in de loop van het promotietraject steeds meer ruimte kwam voor humor en de voortgangsgesprekken niet langer alleen maar beangstigend serieuze gesprekken waren. Jammer vind ik het dan ook dat ik, door het starten van mijn opleiding Interne Geneeskunde in de regio Nijmegen, niet nog meer de Coen leer kennen achter dat meesterlijke brein.

Geert Jan: je weet niet half hoe dankbaar ik ben dat jij één van mijn promotoren wilde zijn! Ondanks de letterlijke afstand (volgens Google Maps $180 \mathrm{~km}$ om precies te zijn) was jij altijd nauw betrokken bij het project en wist je mij van dichtbij te begeleiden. Je nadruk lag daarbij niet alleen op het product maar ook op de persoon die dat product moest produceren. Je luisterend oor en geruststellende woorden hebben me goed gedaan. Je toegankelijkheid en amicaliteit maakte de samenwerking luchtig en plezierig. Ik heb 
bewondering voor het ogenschijnlijke gemak waarmee jij jouw team leidt, ondersteunt en richting geeft. Ik heb veel van je geleerd, maar wat me wellicht het meest zal bijblijven is je constante streven naar duidelijkheid en eenvoud. Je uitspraak "maar hoe zou je het aan je oma uitleggen" dwingt mensen op een ander niveau te denken. Het heeft me geleerd de focus niet uit het oog te verliezen en te blijven bij de kern van het verhaal. Simpel en duidelijk. Dank voor je scherpe blik en positiviteit.

Simone: het aantal keren dat ik jou tijdens het promotietraject heb lastig gevallen is werkelijk ontelbaar. Toch was jij er telkens voor mij. Of het nu ging om logistieke problemen, epidemiologische vragen of het bekijken van (stukjes van) manuscripten: je maakte tijd en gaf me het gevoel altijd tot mijn beschikking te staan. Je tomeloze geduld is goud waard! Ik weet zeker dat je kinderen dat in de toekomst zullen beamen. Ik vond het fijn met je te kunnen sparren wanneer ik vast liep en er even niet meer uit kwam. Ooit heb ik in een enquête geschreven dat je misschien zelfs iets te lief was als co-promoter, en misschien zelfs voor de buitenwereld als geheel. In een wereld waarin iedereen steeds egoïstischer en egocentrischer wordt, probeer(de) jij een verbindende en oplossende factor zijn. Hoe vaak heb ik niet gezien dat jij op de achtergrond (dreigende) problemen oploste? Chapeau daarvoor, hoewel ik me soms nog steeds afvraag of dat ook voldoende werd gezien. Jouw hulp en bijdrage aan dit proefschrift waren in ieder geval onmisbaar en daarvoor kan ik je simpelweg niet genoeg bedanken. Ik hoop dat de toekomst je mag brengen wat je ervan verwacht!

Collega's van De Maastricht Studie: jullie wisten van mijn bevlogen tijd op het onderzoekscentrum een onvergetelijke tijd te maken. Wat hebben we geklaagd en gezeurd, maar vooral ook veel gelachen en lol gemaakt!

Pauline: ons avontuur bij De Maastricht Studie begon zowat tegelijkertijd. Vanaf het begin konden we het goed met elkaar vinden en zaten we op één lijn. Dank voor je luisterend oor, de goede gesprekken, de vele koffiemomenten maar bovenal je vriendschap en gezelligheid. Het was fijn het grootste deel van ons promotietraject als kamergenootjes door te brengen, in goede en minder goede tijden. Je bent een keiharde werker die bergen werk heeft verzet. Het hekelt me dat je daarvoor nog steeds niet de waardering hebt gekregen die je verdient. Ik heb bewondering voor je sterke karakter, doorzettingsvermogen en gedrevenheid en ik weet zeker dat die eigenschappen je nog ver zullen brengen! Dat boekje, dat komt er wel en wordt er één om meer dan trots op te zijn. Blijf bij jezelf en je wordt een fantastisch internist. Dank dat je, nota bene op je verjaardag, achter me wilt staan bij de verdediging van dit proefschrift.

Thomas: onze eerste kennismaking was er één scheurend door het Maastrichtse 
landschap in een verwoede poging jou je trein te laten halen. Die trein heb je niet gehaald, maar het was voor mijn gevoel wel het startpunt van een vriendschap waar ik je dankbaar voor ben. Je was mijn grote voorbeeld van hoe een promotietraject er idealiter uit zou moeten zien en succesvol kan verlopen. Ik bewonder het ogenschijnlijke gemak waarmee jij top- na toppublicatie wist te produceren. Eerlijk is eerlijk, misschien benijdde ik het zelfs een beetje. Je passie voor wetenschap, je scherpe blik en je onuitputtelijkheid zijn lovenswaardig! Toch blijf je bescheiden en sta je niet graag op de voorgrond, iets wat je mijns inziens siert. Ik wil je bedanken voor het delen van je inhoudelijke kennis. Temeer wil ik je bedanken voor de gezellige momenten die we samen met onze partners in Eindhoven hebben beleefd. Ik vind het jammer dat we momenteel niet meer in dezelfde stad wonen, maar ondanks de afstand heb ik er alle vertrouwen in dat we elkaar nog vaak zullen zien. Het doet me deugd te weten dat ook jij, net als Pauline, achter me staat bij de verdediging van dit proefschrift.

Remy: ik denk dat ik ruim een jaar met mijn promotietraject bezig was toen jij startte bij De Maastricht Studie. Vrijwel meteen had ik in jou een partner in crime gevonden. Menig collega en lid van het Management Team moet ons bij tijd en wijle hebben vervloekt. Bij elk overleg of bespreking waren wij veelal de personen met de kritische blik, de vervelende vragen of de sarcastische uitspraken. Toegegeven, althans voor mij, was het soms niet meer dan een sport. Maar zijn het niet de dwarsliggers die het spoor doen rechtlopen? Ik hoop dat onze kritische houding niet alleen voor onszelf als uitlaatklep heeft gewerkt, maar uiteindelijk ook een positieve bijdrage heeft geleverd aan het verbeteren van (de logistiek rondom) De Maastricht Studie. Remy, ons gevoel voor humor (alsmede onze schoenkeuze) lag op één lijn! Ok, droge humor, maar wat hebben we gelachen. Dank voor de leuke tijd en de gezellige lunches aan het einde van mijn promotietraject. Succes met de allerlaatste loodje aan je eigen proefschrift, hetgeen volgens mij een meesterwerk gaat worden.

Julianne: jouw project startte enkele maanden na het mijne. Toch heeft het volgens mij enige tijd geduurd voordat we elkaar leerden kennen en wisten wat we aan elkaar hadden. Je bent recht door zee en neemt geen blad voor de mond, en dat kan ik zeer waarderen. Dat, gecombineerd met je noordelijke norsheid, maakte je wat mij betreft tot een gewaardeerd collega. Inmiddels heb je je eigen proefschrift verdedigd en heb je de wetenschap (tijdelijk) achter je gelaten om je te richten op een carrière in de journalistiek of een combinatie van beide. Veel succes in de toekomst gewenst! Nu we beiden in regio Nijmegen wonen gaan we elkaar zeker nog vaak treffen.

Het laatste deel van dit dankwoord wil ik wijden aan de belangrijkste personen in mijn leven. Zonder hen had ik nooit gestaan waar ik nu sta en daar ben ik hun zeer erkentelijk 
voor. Soms baal ik van het feit dat ik zoveel van mijn vrije tijd heb opgesnoept om dit proefschrift voor elkaar te krijgen terwijl ik die tijd ook met hun had kunnen (en willen) doorbrengen. Gedane zaken nemen geen keer, maar ik hoop dat jullie trots zijn op het eindresultaat.

Pappa en mamma: zonder jullie was dit boekje er, letterlijk én figuurlijk, nooit geweest. Jullie hebben er altijd alles aan gedaan om het ons aan niks te laten ontbreken. Hierbij hebben jullie jezelf menigmaal weggecijferd. Ik kan jullie niet genoeg bedanken voor de kansen die jullie ons hebben gegeven. Jullie hebben ons geleerd het beste uit onszelf naar boven te halen. Dank voor jullie rotsvaste vertrouwen in mij en mijn kunnen. Misschien maak ik niet altijd de keuzes waar jullie direct achter staan, maar weet dat ik onvoorwaardelijk veel van jullie houd en altijd aan jullie denk!

Claudia en Simone, oftewel mijn zussen: kleine broertjes worden groot. Met weemoed denk ik soms terug aan die goede oude tijd: schommelen in de tuin, skeeleren door de buurt, en flippo-en op het schoolplein. Tegenwoordig hebben we allemaal ons eigen stekkie en zijn we alle drie onze eigen weg gegaan. Toch zien we elkaar regelmatig en dat doet me ontzettend goed. Dank voor al jullie steun de afgelopen jaren en de interesse die jullie hebben getoond in mijn proefschrift. Het geeft me een goed gevoel te weten dat ik altijd bij jullie terecht kan. Orhan, ik ben blij dat Simone jou heeft leren kennen. Je bent een fantastische schoonbroer met het hart op de juiste plaats.

Ook mijn schoonfamilie wil ik bedanken voor hun steun, vertrouwen, interesse en waardering. Dank voor jullie toegankelijkheid en gezelligheid. Het doet me goed te weten dat ook jullie altijd voor mij klaar zullen staan.

Marleen: jouw glimlach werkt ontwapenend, je aanwezigheid laat me relativeren en bij jou zijn is simpelweg het fijnste wat er is. Retrospectief betreur ik de enorme hoeveelheid vrije tijd die in dit proefschrift zit. Tijd die ik veel liever met jou had besteed. Immers: zonder jou zou dit boekje me gestolen kunnen worden. Dank voor je onvoorwaardelijke steun, vertrouwen en liefde! Vol verwachting kijk ik uit naar de avonturen die gaan komen. Er ligt veel moois in het verschiet, zowel gepland als ongepland. Marleen, liefste schat, ik houd van jou!

Hoewel ik weet dat de werkelijk niet in one- of two-liners is samen te vatten, komt deze aardig in de buurt: "Individually, we are one drop. Together we are an ocean" (Ryunosuke Satoro). Samen staan we sterk en kunnen we de wereld aan!

Stefan 
CURRICULUM VITAE 


\section{Curriculum vitae}

Stefan Geijselaers was born on October 19, 1987 in Maastricht, the Netherlands. After graduating from secondary school (Sint-Maartenscollege, Maastricht) in 2005, he started his medical training at Maastricht University. In 2011, Stefan obtained his medical degree and started working as a PhD student at the same university. The research described in this thesis was conducted under supervision of Prof. Coen Stehouwer (Maastricht University Medical Centre + ) and Prof. Geert Jan Biessels (University Medical Centre Utrecht). Since March 2016, Stefan is working as a resident in Internal Medicine at the Jeroen Bosch Hospital, Den Bosch, the Netherlands. 
